\title{
A discriminative approach to harmonic emission assessment
}

\section{B Peterson \\ (D)orcid.org/0000-0001-8282-1192}

Thesis accepted in fulfilment of the requirements for the degree Doctor of Philosophy in Electrical Engineering at the North-West University

Promoter:

Co-Promoter:

Co-Promoter:
Prof APJ Rens

Dr G Botha

Prof J Desmet

Graduation: June 2021

Student number: 26583089 


\section{PREFACE}

All of the work presented in this thesis was born from the integration of renewable energy sources into the South African electrical network. Grid code compliance processes forced the electrical utility to rethink their traditional approach on how harmonic emission assessments were done. The constraint that they faced was the measurement of harmonic emission from a Renewable Power Plant (RPP) by using only a 10-min rms value.

In the beginning, the CIGRE/CIRED C4.109 statistical method was deemed to be the appropriate method to prove compliance and was written into grid code documents as the main method applicable for harmonic emission assessment. As the method was applied to various RPPs at various network connection points, it highlighted major limitations in the method that resulted in disputes between the utility and the RPP.

Research presented in this thesis showed that harmonic emission assessment requires a more complex approach to ensure that RPPs only account for their harmonic emissions and that they are treated fairly as grid code compliance within the $10-\mathrm{min}$ rms constraint meant that all harmonic current values was deemed to be emission.

The research done in this thesis has subsequently been used to change the grid code documents to include various proven harmonic emission assessment methods by application of aggregated harmonic phasors. The student led the utility team that developed the revised grid code documents to be used by RPPs. At the time of compiling this thesis the changes was accepted by industry and RPPs with the awaiting final approval by the local electricity regulator.

The research work was done in conjunction with North West University and was not sponsored by the local utility. The research is original and is based on papers published in recognised publications by the student.

\section{ACKNOWLEDGEMENTS}

This Thesis is dedicated to my late mentor Mr Frederik "Das" Venter. Without his guidance I would not be on the path that I am today, and I am forever thankful.

I would like to acknowledge the following people for their encouragement and unwavering support;

- Thank you to the Lord almighty for the perseverance and the courage to take on a $\mathrm{PhD}$.

- Mr Jan Hendrik Hattingh, Prof. Jan Desmet (co-promoter), Prof. Jan Meyer, Dr Ana Blanco and Dr Ulrich Minnaar for your guidance and expertise.

- My managers at Eskom Mr Rakesh Ragoonathun, Mr Andries Cornelius (retired) and Mr Nico Kleynhans for allowing me latitude during this time

- To my co-promotor Dr Gerhard Botha, for the interesting discussions and for pushing me to go beyond what I thought I could do,

- To Prof Johan Rens, for firstly filling up my personal memory book, secondly for putting up with my nagging and thirdly for giving me a platform to develop and to grow from strength to strength.

- Last my wife Gillian, thank you the guidance and the support that kept me focused in achieving my goal. 


\section{ABSTRACT}

Harmonic emission assessment of a Renewable Power Plant (RPP) for grid code compliance, is a complex task that requires a fair and pragmatic approach. RPPs should only account for the harmonics they emit, i.e. those which negatively affect the harmonic voltage at the Point of Connection (PoC).

Most of the harmonic emission assessment methods require the use of measured harmonic phasors.

However, harmonic phasor measurements taken for more than 24 hours, result in an enormous volume of data that is cumbersome to analyse. Power Quality (PQ) measurements is typically done over days (in some cases over 7 days [1]), which is why power quality data is aggregated to reduce the volume of data.

Aggregation of rms data is a known concept that is detailed in the IEC 61000-4-30 power quality measurement standard. Depending on the aggregation time interval selected, datasets of 3-sec, 10mins or 120-min time intervals can result. The challenge is that only rms data are retained in this standard, with phase angle information being discarded.

With the constraint of using a 10-min rms value, the CIGRE/CIRED joint working group C4.109 developed a statistical approach that makes use of harmonic voltage and harmonic current scatterplots with reference impedances of the upstream network and the downstream nonlinear load to qualitatively evaluate which party is the major contributor towards the harmonic distortion measured at the Point of Connection' 1 (PoC).

The CIGRE/CIRED C4.109 statistical method (in this thesis) was found to be inadequate in conclusively indicating which harmonics are being emitted or being absorbed by a PV plant when only one measurement point is considered at the PoC. By application of synchronised and coherent measurements at multiple points, the method was improved to discriminate harmonic emission within the 10-minute rms constraint.

RPPs or nonlinear loads may not be able to conduct a multiple measurement point campaign, as they only have access to their PoC. Therefore, an alternative approach is required to evaluate their harmonic emissions. Aggregation of harmonic phase angles, in line with IEC61000-4-30 principles, is a new concept that results in a reduced dataset consisting of aggregated harmonic phasors, that can be used to continuously assess harmonic emission at a single measurement point.

Application of aggregated harmonic phasors to a PV plant undergoing grid code compliance, found that the discrimination of harmonic emission at a single measurement point can be done through the Range of Secondary Cancelation (RSC) technique, which has thus far only been discussed in theory.

Confidence in the RSC technique and aggregated harmonic phasors were further confirmed with field measurements (over a 60-day period) at an industrial site with the aim to monitor harmonic filter performance. It was found by application of the RSC technique, that one of the filter banks did not perform as designed, while the other filter banks effectively reduced the harmonic voltage at the PoC.

The RSC technique achieved discrimination of harmonic emission values, however the actual harmonic emission value used for determining compliance to emission limits is to be evaluated.

\footnotetext{
${ }^{1} \mathrm{PoC}$ is defined as the reference point on the electric power system where the user's electrical facility is connected
} 
Application of the IEC 61000-3-6 general summation law was found to be too conservative due to the use of summation factors in the summation of harmonic distortion. In a real network harmonic distortion summation is dynamic especially when other non-linear loads are connected to the Point of Common Coupling (PCC). The "prevailing phase angle" value was used as a representative value of the harmonic dataset to result in a more accurate harmonic emission assessment in this integrated network.

The harmonic emission assessment methods mentioned above makes use of a fixed time aggregation interval, however electrical supply networks are not static and harmonic generation patterns from non-linear load changes dynamically over time. A fixed aggregation time interval may thus not capture the dynamic behaviour of the RPPs or nonlinear loads.

A new concept was developed and tested to dynamically select the aggregation time interval to ensure meaningful aggregated harmonic phasors that capture the harmonic distortion from a nonlinear load with considerable variations. This approach achieved a reduction in the dataset to be considered for harmonic emission assessment whilst capturing the dynamic nature of the nonlinear load.

In this thesis, it was demonstrated that;

- discrimination of harmonic emission values was achieved to only account for harmonic distortion being emitted from a non-linear load,

- harmonic emission assessment requires multiple measurement points when only rms values are considered,

- aggregated harmonic phasor measurements drastically reduce the size of dataset used for harmonic emission assessment when more than 24 hours of data is considered,

- harmonic emission can be assessed continuously by applying the aggregation of harmonic phasors and the dynamic selection of the aggregation time interval.

The above-mentioned tools will aid a pragmatic engineer to conduct a successful harmonic emission assessment that accurately reflects the harmonic contribution of the RPP in a fair manner.

Key terms: harmonic emission, prevailing phase angle, dynamic aggregation, harmonic distortion, harmonic assessment 


\section{TABLE OF CONTENTS}

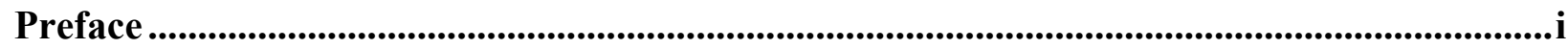

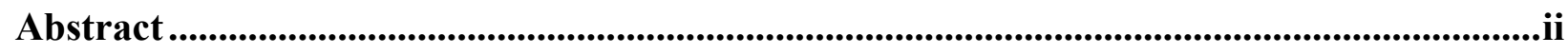

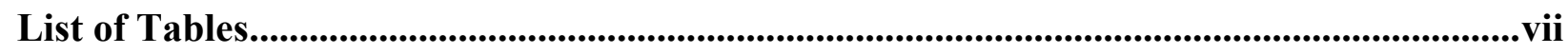

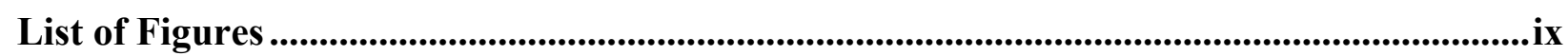

List of peer-reviewed conference publications .................................................................xiii

List of peer-reviewed journal publications .......................................................................

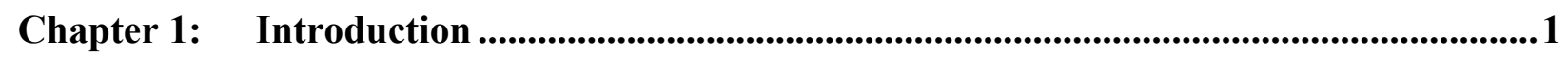

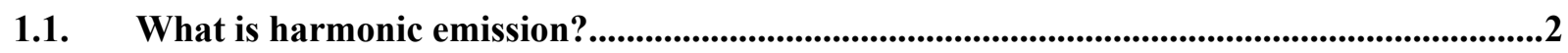

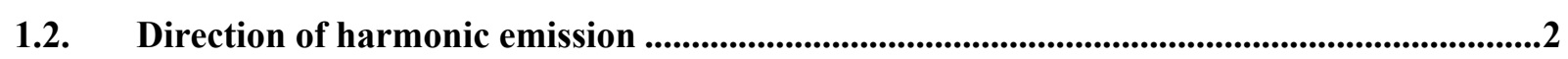

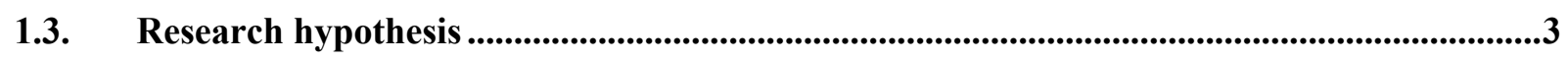

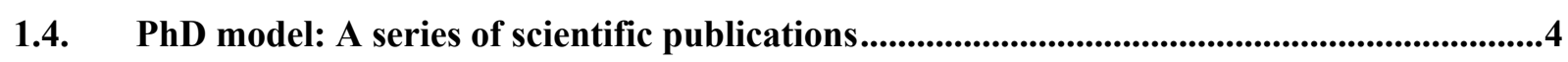

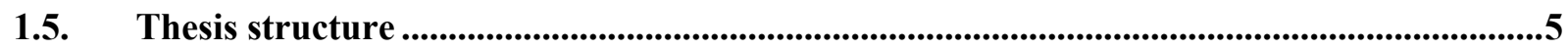

Chapter 2: Harmonic Emission: What is currently known ......................................................7

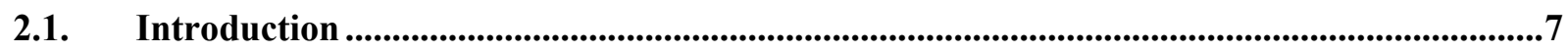

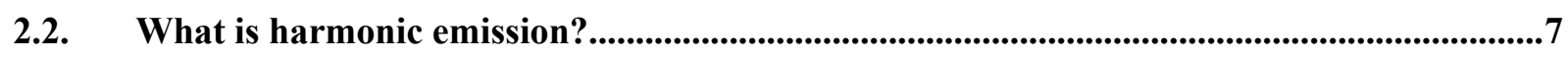

2.3. Renewable energy sources and voltage harmonic distortion ..............................................8

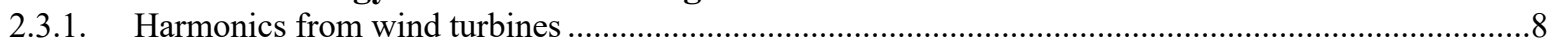

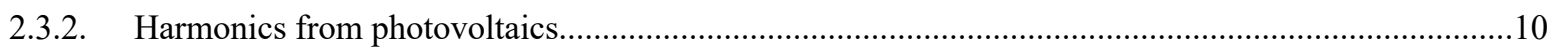

2.4. Why harmonics are a concern for electrical utilities .................................................................11

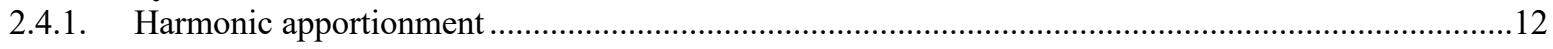

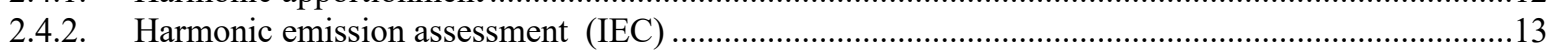

2.5. Comparison of harmonic distortion localisation methods....................................................14

2.5.1. Harmonic active power in the localisation of harmonic sources ..................................................15

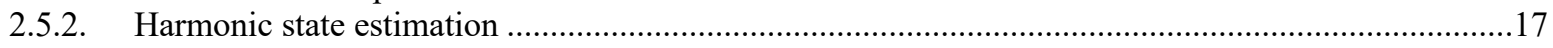

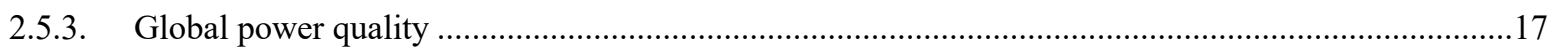

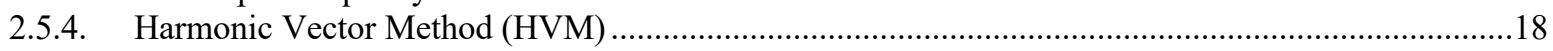

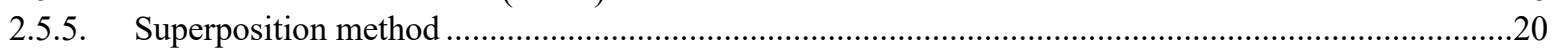

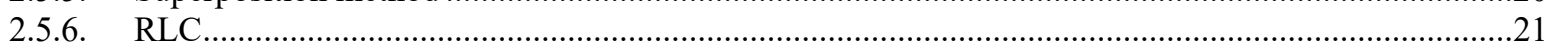

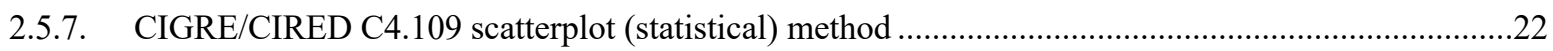

2.5.8. Range of secondary cancellation: when does harmonic matter? ......................................................24

2.6. The measurement of harmonics......................................................................................................26

2.6.1. IEC 61000-4-30 and IEC 61000-4-7: How harmonics are measured by engineers .............................26

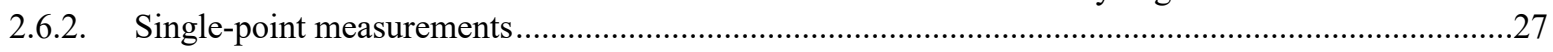

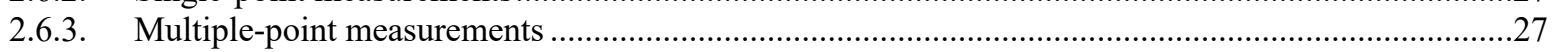

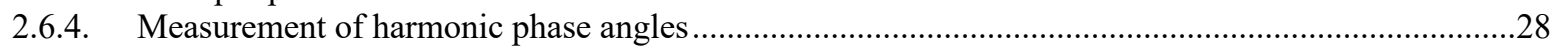

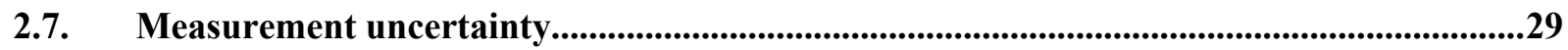

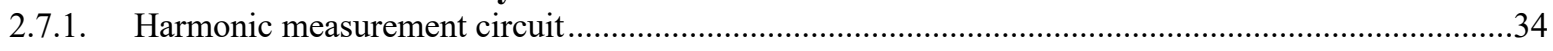

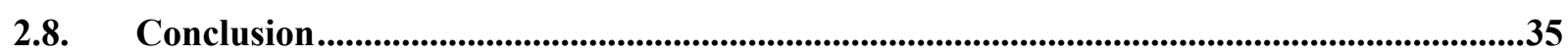

Chapter 3: Verification of harmonic emission assessment methodologies........................38

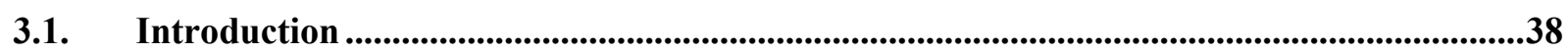

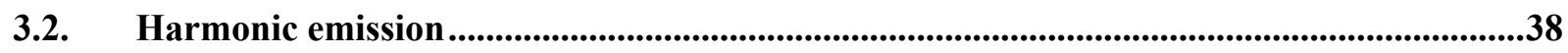


3.3. Harmonic emission assessment using harmonic active power with one source of harmonic distortion

3.4. Harmonic emission assessment using harmonic active power with more than source of harmonic distortion

3.5. Harmonic emission assessment by the CIGRE C4.109 scatter plot method........................46

3.6. Harmonic emission assessment by the Harmonic Vector Method ..........................................48

3.7. Harmonic emission assessment by the range of secondary cancellation between harmonic

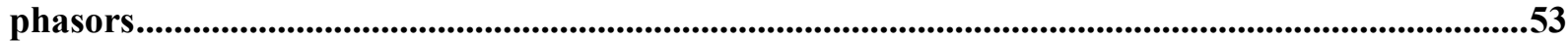

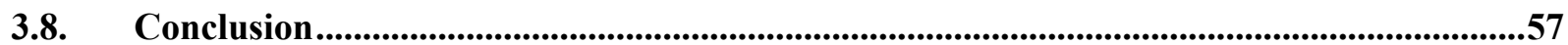

Chapter 4: Limitations for field application of harmonic emission assessment ................58

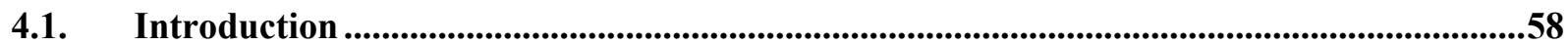

4.2. RMS harmonic data analysis when using single-point measurements..............................59

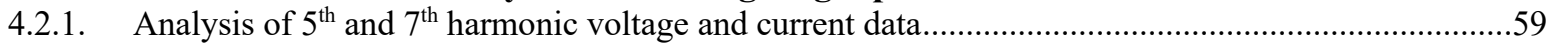

4.2.2. Application of CIGRE/CIRED C4.109 scatterplot method...............................................................63

4.2.3. Harmonic emission calculation based on CIGRE/CIRED C4.109 ..................................................66

4.2.4. Conclusion on harmonic emission assessment using single-point measurements ..............................66

4.3. Evaluation of synchronised measurements across an impedance between the PoC and the PCC 67

4.3.1. Evaluation of CIGRE/CIRED C4.109 scatterplot method using synchronised measurement .............70

4.3.2. Harmonic emission using synchronised measurements......................................................................

4.4. Measurement uncertainty................................................................................................................72

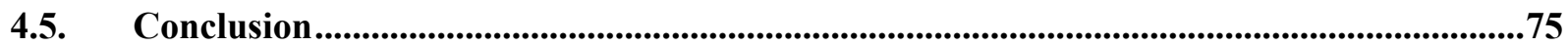

Chapter 5: Assessment of harmonic emission using aggregated harmonic phasors:

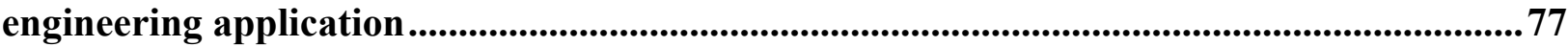

5.1. Introduction .......................................................................................................................................77

5.2. Aggregation of harmonic phasors...................................................................................................77

5.3. Opportunity for the prevailing phase angle...................................................................................79

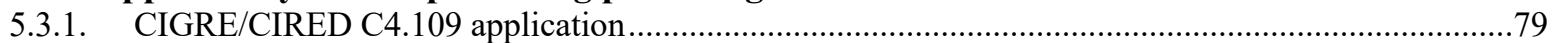

5.3.2. Analysis of harmonic field data based on aggregated harmonic phasors ..........................................82

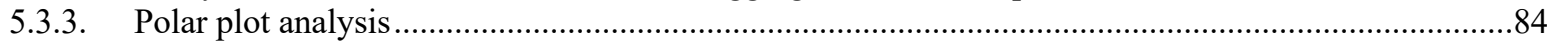

5.4. How much of harmonic emission is contributed by the PV plant? ..........................................87

5.5. Field evaluation: range of secondary cancellation of harmonic phase angles ......................89

5.5.1. Secondary cancellation of harmonic phase angles at a PV plant (RSC principle) ...............................90

5.5.2. Harmonic filter performance assessment.......................................................................................

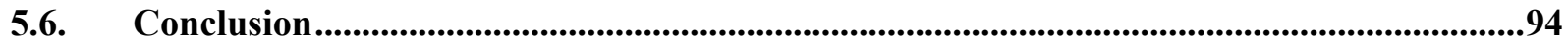

Chapter 6: Application of the prevailing phase phasor in a high voltage network..........96

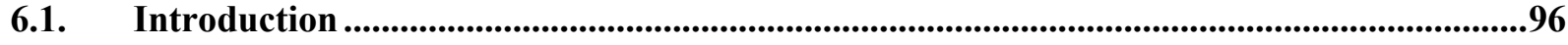

6.2. Evaluation of harmonic emission at a distribution substation with multiple nonlinear loads connected to the same busbar.................................................................................................96

6.3. Harmonic emission assessment of PV plants under dynamic conditions............................102

6.3.1. RSC calculations for PV plants under dynamic conditions ..........................................................102

6.3.2. A comparison between the prevailing phase angle method and the IEC method for harmonic emission

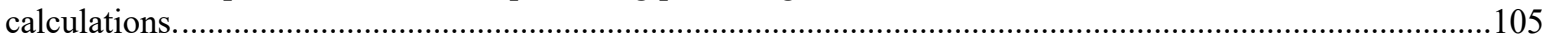

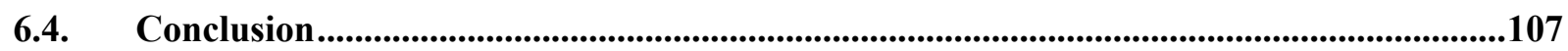


Chapter 7: Consideration on the aggregation time interval ...............................................109

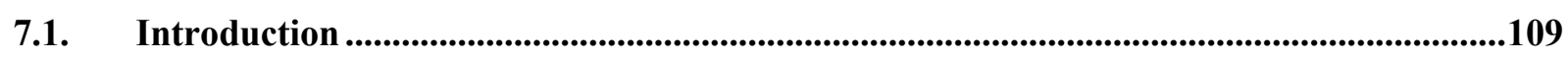

7.2. Evaluation of time aggregation intervals ...............................................................109

7.3. Impact on level of prevalence ..........................................................................................114

7.4. Dynamic selection of aggregation time interval ...........................................................................114

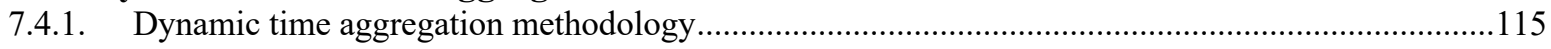

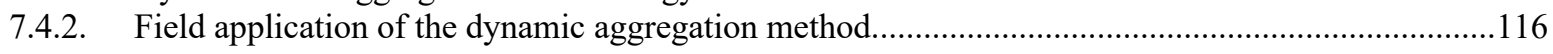

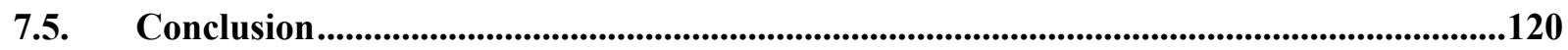

Chapter 8: Conclusion...........................................................................................................122

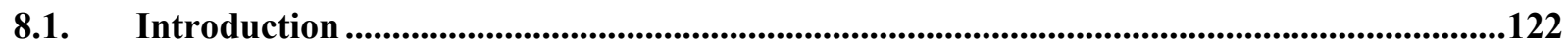

8.2. The need for a discriminative approach to harmonic emission assessment ......................122

8.3. Assessing the hypothesis ..................................................................................................................122

8.3.1. Analysis and modelling of harmonic emission from first principles in an interconnected network with more than one source of harmonic waveform distortion are reported. .......................................................123

8.3.2. The metrological requirements and constraints of measuring both voltage and current harmonics are derived. 123

8.3.3. Can the relative contribution to harmonic distortion be quantified based on rms harmonic measurements obtained from IEC 61000-4-30 Class A edition 3 compliant instrumentation?...............

8.3.4. Can existing harmonic assessment techniques be improved to sufficiently discriminate between harmonic emission contributed by different Renewable Power Plants (RPP) or by a specific distorting load, or

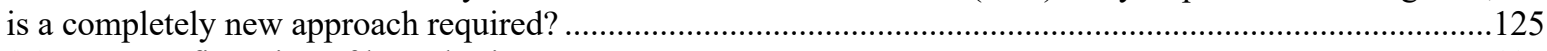

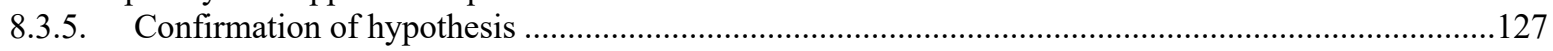

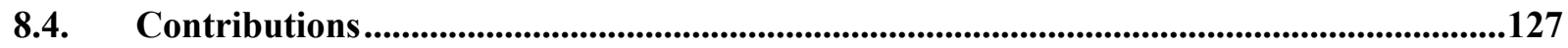

8.5. Significance of results...............................................................................................................128

8.6. Recommendations for RPPs .......................................................................................................129

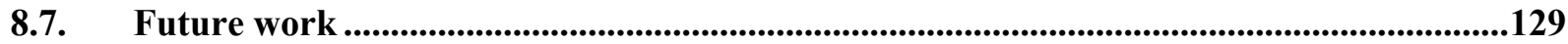

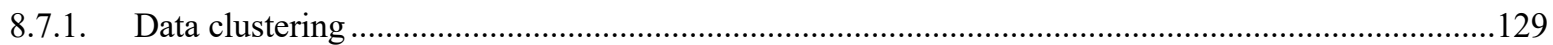

8.7.2. Complex harmonic active power in the presence of multiple non-linear loads......................................130

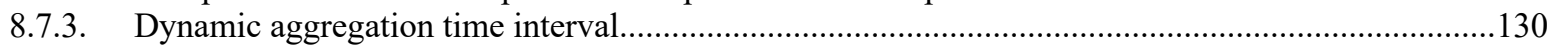

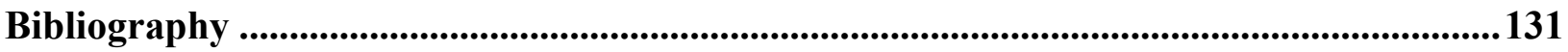

Appendix A: Verification of additional harmonic orders ......................................................... 139 


\section{LIST OF TABLES}

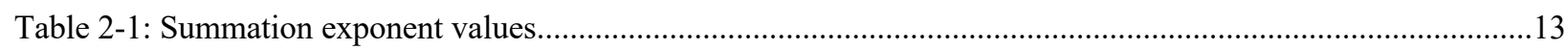

Table 2-2 Various methods for harmonic direction determination [15] ..................................................................15

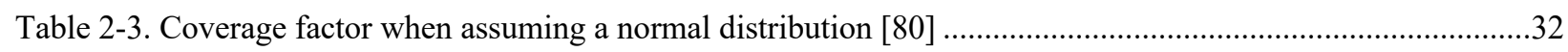

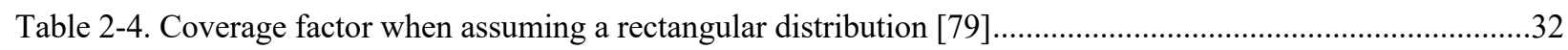

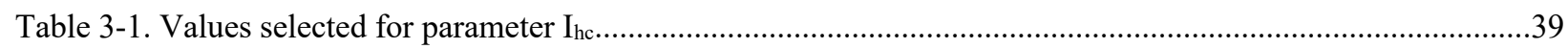

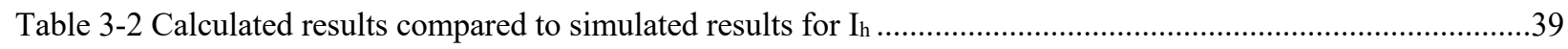

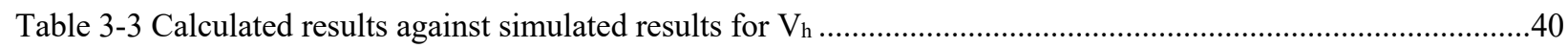

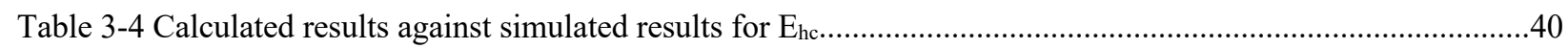

Table 3-5. Inputs used when harmonic source is in both the network and the load ............................................47

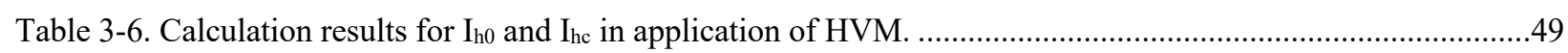

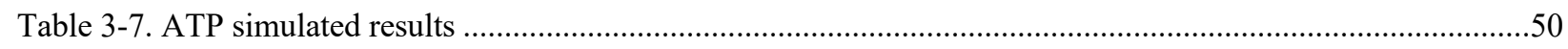

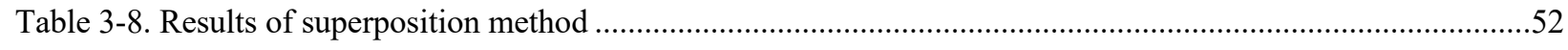

Table 3-9 RSC $5^{\text {th }}$ harmonic values: harmonic source in the load. .......................................................................54

Table 3-10 RSC calculated values for the $5^{\text {th }}$ harmonic when harmonic source is in the upstream network..............55

Table 3-11 RSC calculated values for the $5^{\text {th }}$ harmonic when harmonic source is in the upstream network (reduced

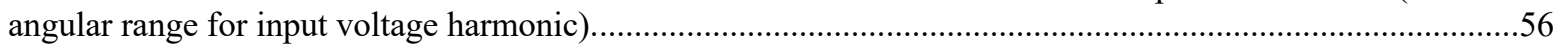

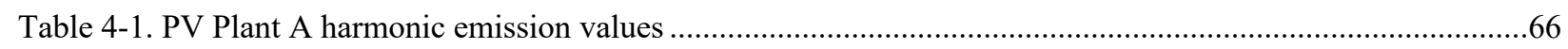

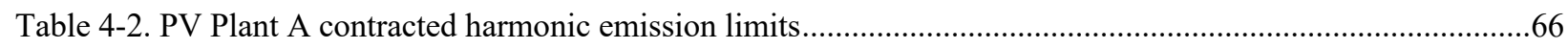

Table 4-3. Results of the $5^{\text {th }}$ harmonic emission assessment using 3 different methods ..........................................72

Table 4-4. Results of the $7^{\text {th }}$ harmonic emission assessment using 3 different methods .........................................72

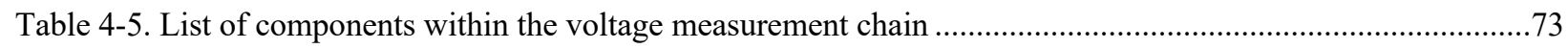

Table 4-6. List of components within the current measurement chain.....................................................................73

Table 4-7. Combined standard uncertainty for measurement circuit in Figure 4-15 .............................................74

Table 4-8. Expanded combined standard uncertainty for the measurement circuit in Figure 4-15..........................74

Table 4-9. Results of the $5^{\text {th }}$ harmonic emission assessment using 3 different method and with measurement

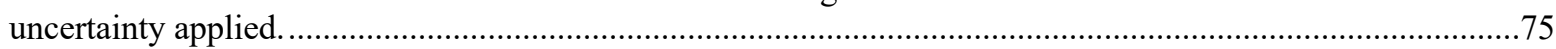

Table 4-10. Results of the $7^{\text {th }}$ harmonic emission assessment using 3 different method and with measurement

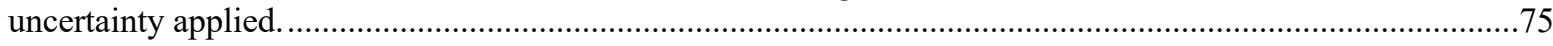

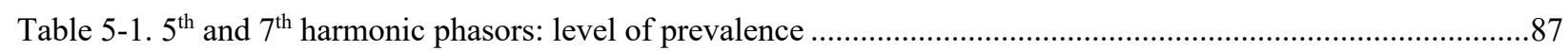

Table 5-2. Levels of prevalence for the day and night time for the $5^{\text {th }}$ harmonic...........................................87

Table 5-3. Calculated values and results for harmonic current RSC .............................................................90

Table 5-4. Calculated values and results for harmonic current RSC for the $3^{\text {rd }}$ and $5^{\text {th }}$ harmonic ...........................93

Table 6-1. Level of prevalence for current harmonic measurement points R1 to R5........................................101

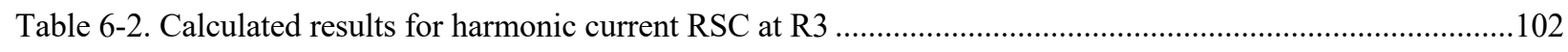

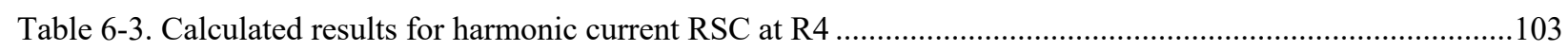

Table 6-4. Harmonic emission results from PV Plant at R3 with all data considered..........................................105

Table 6-5. Harmonic emission results from PV Plant at R4 with all data considered..........................................106

Table 6-6. Harmonic emission results from PV Plant at R3 with only discriminated data considered.....................106

Table 6-7. Harmonic emission results from PV Plant at R4 with only discriminated data considered.....................107 
Table 7-1. Level of prevalence for Site A and Site B for different aggregation time intervals. .114

Table 7-2 Prevailing phasor values for different aggregation time intervals ...... .114

Table 7-3. Number of aggregation time interval points for the selected levels of prevalence...... 


\section{LIST OF FIGURES}

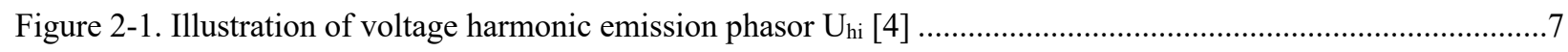

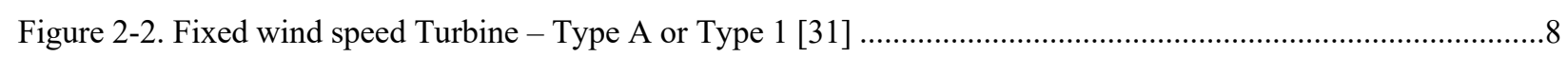

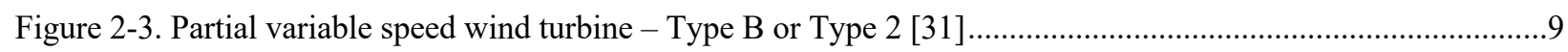

Figure 2-4. Variable speed wind turbine with partial power converter - Type C or Type 4 [31] ..............................

Figure 2-5. Variable speed wind turbine with Full power converter - Type D or Type 4 [31] ................................

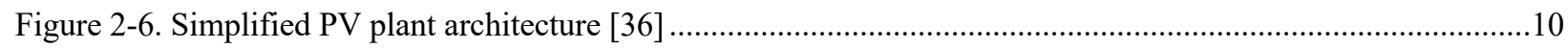

Figure 2-7. Current THD against Power output of a PV inverter at a local PV plant [43] over a 24 hour period .......11

Figure 2-8. Illustration of basic Power Quality management adapted from [4] ......................................................12

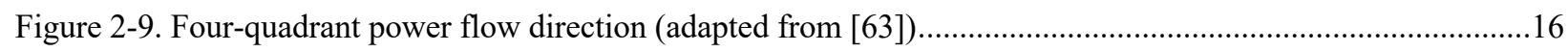

Figure 2-10. Norton equivalent circuit for utility and customer networks [13]....................................................18

Figure 2-11. Example of utility and customer contributions to the measured current phasor at the PCC [13]...........19

Figure 2-12. Customer and utility harmonic current contribution using superposition principles [13] ....................20

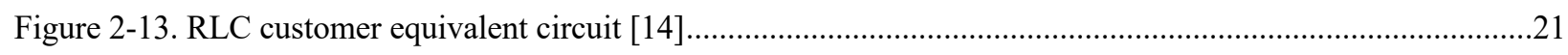

Figure 2-14. Equivalent network diagram for the definition of the harmonic emission level at the PoC [19] ...........22

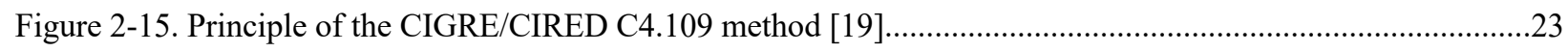

Figure 2-16. Simplified single-line equivalent circuit (adapted from [71]) ........................................................24

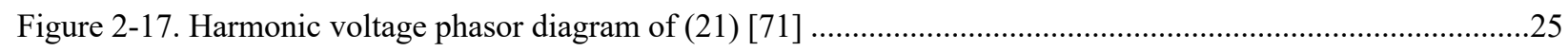

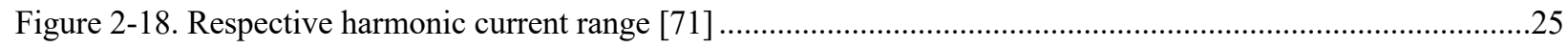

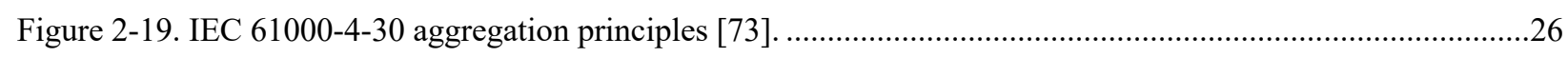

Figure 2-20. Multiple-point measurement system (adapted from [76]) .........................................................28

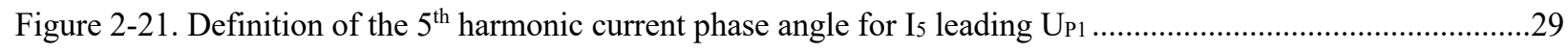

Figure 2-22. Definition of the $5^{\text {th }}$ harmonic current phase angle for $I_{5}$ lagging Up1 ............................................29

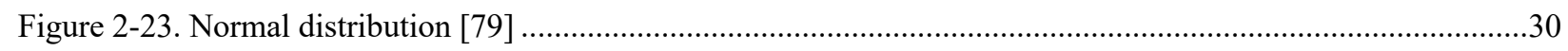

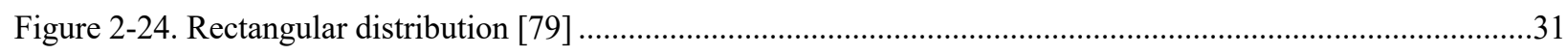

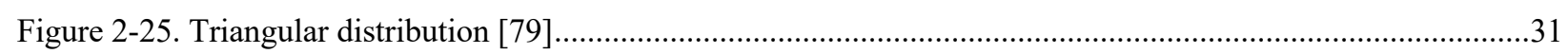

Figure 2-26. Extract from [83] showing normalised magnitude of the frequency response of voltage transformers at

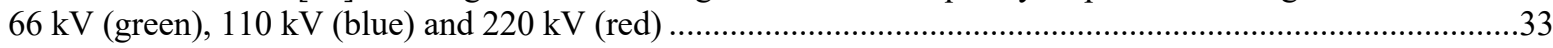

Figure 2-27. Extract from [83] showing phase angle difference of the frequency response of voltage transformers at

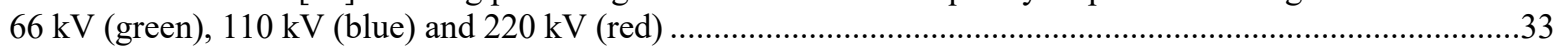

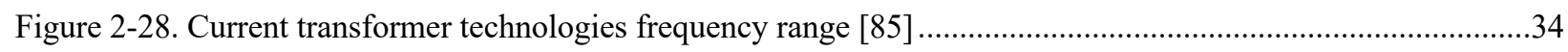

Figure 2-29. Typical metering system for billing and secondary measurements [86]...........................................35

Figure 3-1. Voltage harmonic emission diagram for harmonic current input $1.4 \angle 30^{\circ} \mathrm{A}$ for the $5^{\text {th }}$ harmonic ..........41

Figure 3-2. Complex harmonic active power with only load as a harmonic source.............................................42

Figure 3-3. Complex harmonic active power with only network as a harmonic source ..........................................42

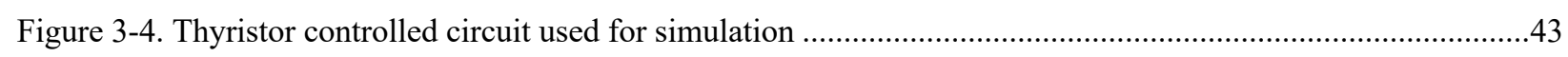

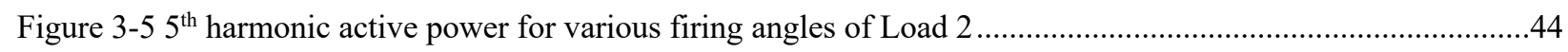

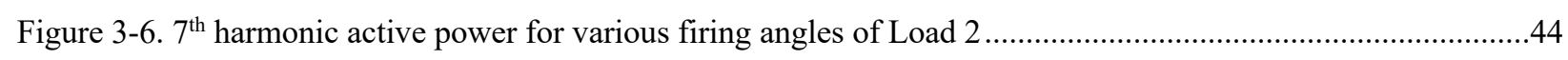

Figure 3-7. CIGRE C4.109 scatterplot when no harmonic distortion exist in upstream network .............................46 
Figure 3-8. CIGRE C4.109 scatterplot when no harmonic distortion exist in the load

Figure 3-9. CIGRE C4.109 scatterplot when harmonic sources exist in the upstream network and load side ...........47

Figure 3-10. Norton equivalent circuit diagram used to simulate the HVM. ..................................................48

Figure 3-11. Example of utility and customer contributions to the measured current phasor at the PoC [13] ...........50

Figure 3-12. Visualisation of the network harmonic current and the load harmonic current for Norton equivalent circuits when $\mathrm{I}_{\mathrm{h}}=1.4 \angle-130.6^{\circ}$ A using the harmonic vector method.

Figure 3-13. Illustration of superposition method of the harmonic contributions from the load and the network to a single measurement point.

Figure 3-14. Range of secondary cancellation with harmonic current values: Harmonic source exists in load ..........54

Figure 3-15. Range of secondary cancellation with harmonic current values: Harmonic source exists in network....55

Figure 3-16. Range of secondary cancellation with harmonic current values: Harmonic source exists in network with reduced angular range of harmonic voltage.....

Figure 4-1 PV plant harmonic emission assessment, PoC defined at the $22 \mathrm{kV}$ connection downstream of the overhead line...

Figure 4-2. $5^{\text {th }}$ harmonic voltage vs $5^{\text {th }}$ harmonic current measurements at PV plant........................................60

Figure $4-3.7^{\text {th }}$ harmonic voltage vs $7^{\text {th }}$ harmonic current measurements at PV plant............................................61

Figure 4-4. $5^{\text {th }}$ harmonic voltage vs $5^{\text {th }}$ harmonic current measurements at PV plant with only positive harmonic active power considered.

Figure 4-5. $7^{\text {th }}$ harmonic voltage vs $7^{\text {th }}$ harmonic current measurements at PV plant with only positive harmonic active power considered....

Figure 4-6. Frequency sweeps for the network under grid code compliance ......................................................64

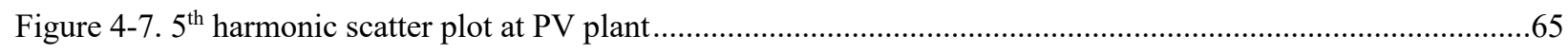

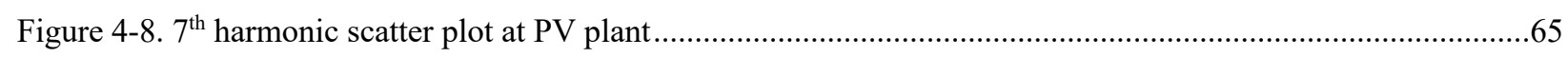

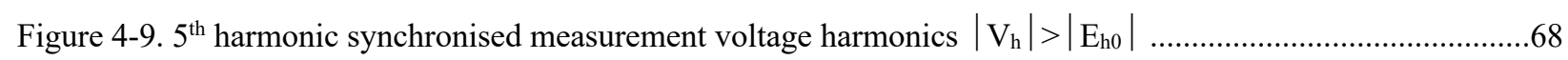

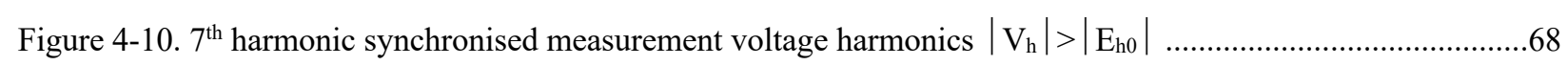

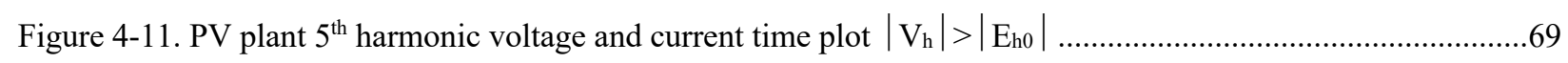

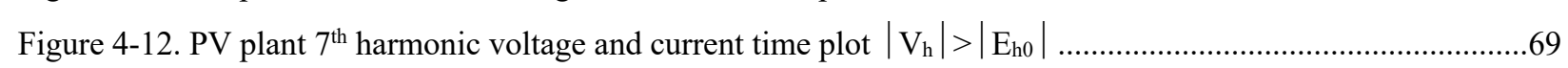

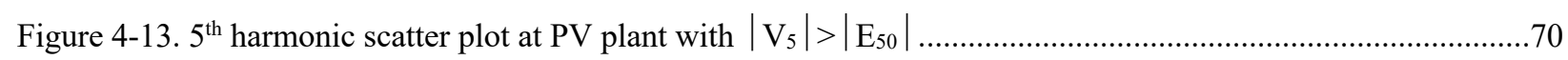

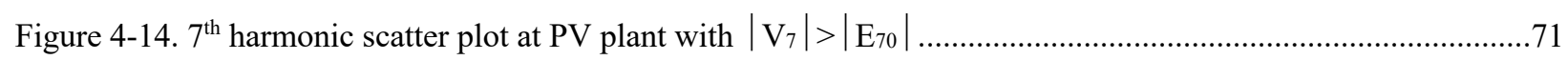

Figure 4-15. Simplified measurement circuit and the uncertainty error for each component contributing to the

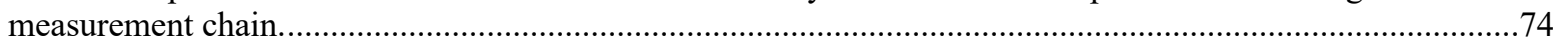

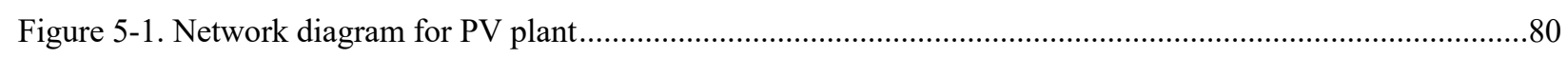

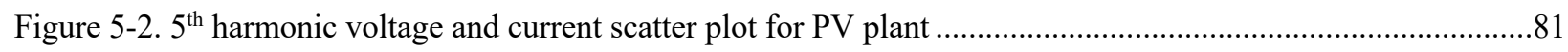

Figure 5-3. $7^{\text {th }}$ harmonic voltage and current scatter plot for PV plant ................................................................8

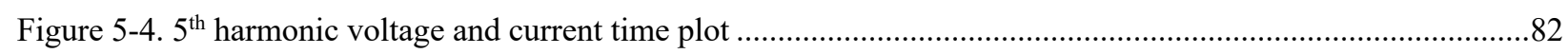

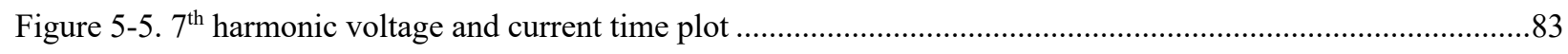

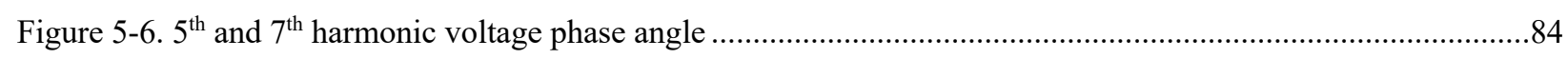

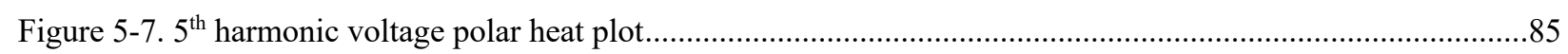

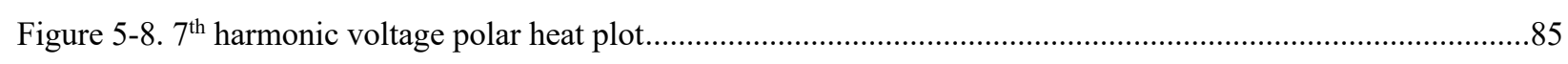

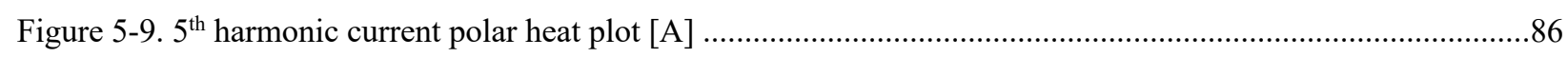

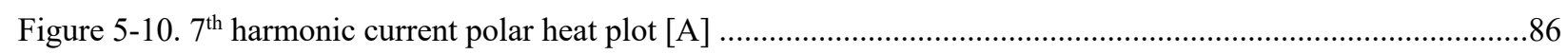

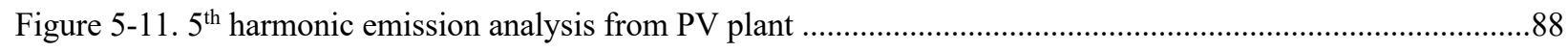


Figure 5-12. $7^{\text {th }}$ harmonic emission analysis from PV plant

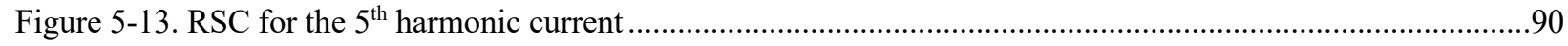

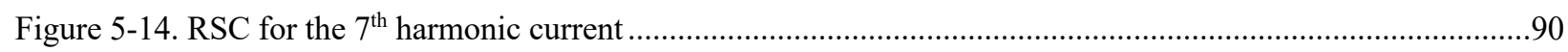

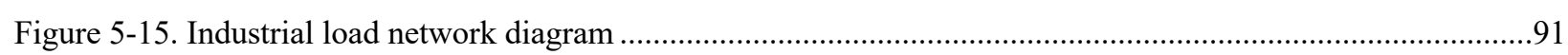

Figure 5-16. $3^{\text {rd }}$ harmonic voltage and current magnitude and phase angle plots ................................................99

Figure 5-17. $5^{\text {th }}$ harmonic voltage and current magnitude and phase angle plots ................................................99

Figure 5-18. $11^{\text {th }}$ harmonic voltage and current magnitude and phase angle plots ................................................92

Figure 5-19. $13^{\text {th }}$ harmonic voltage and current magnitude and phase angle plots .............................................93

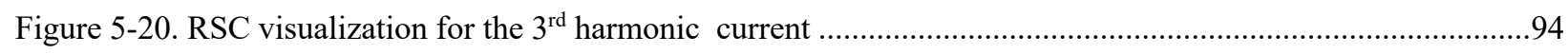

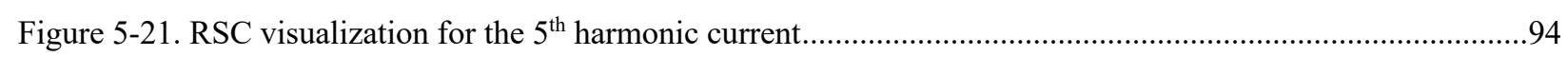

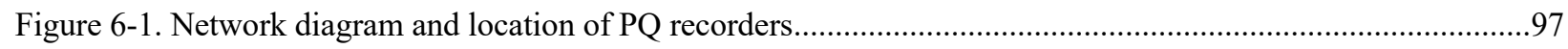

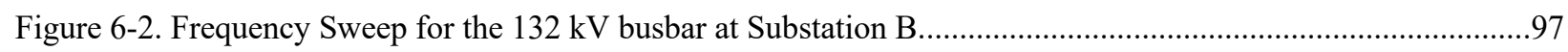

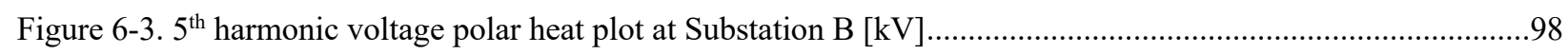

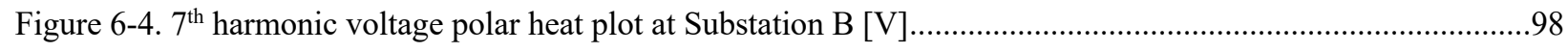

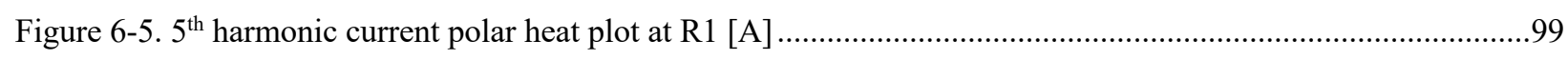

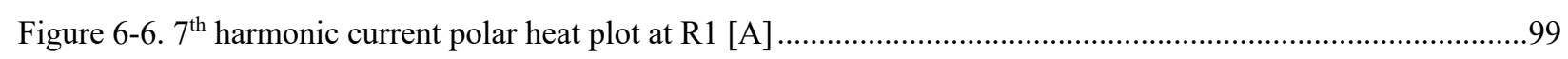

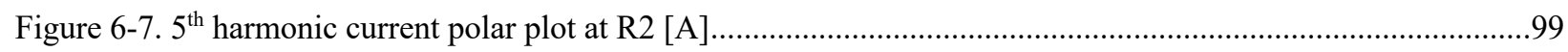

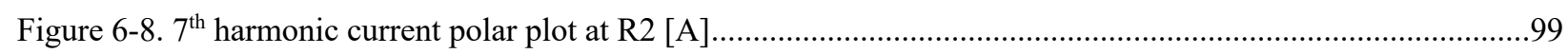

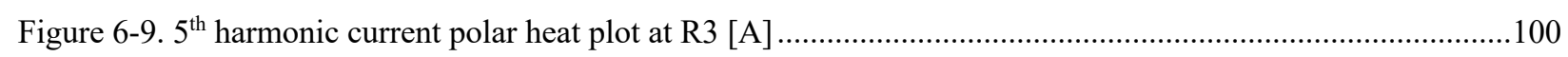

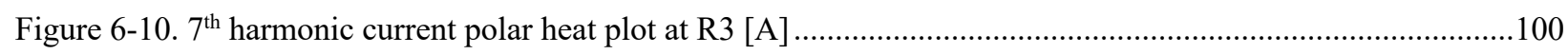

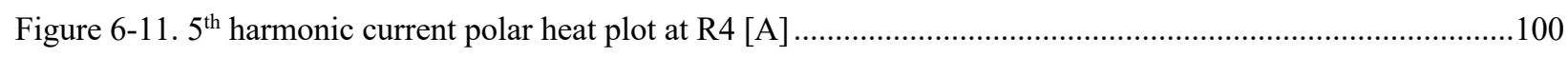

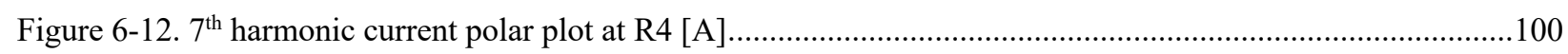

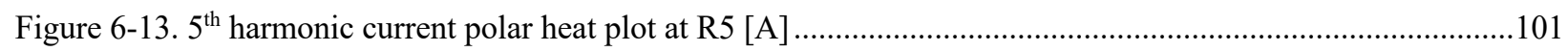

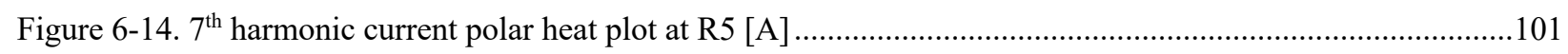

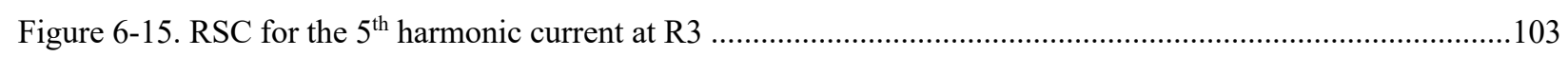

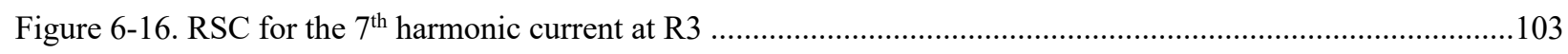

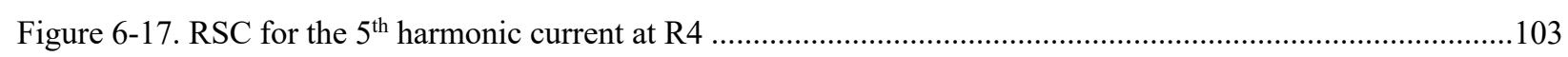

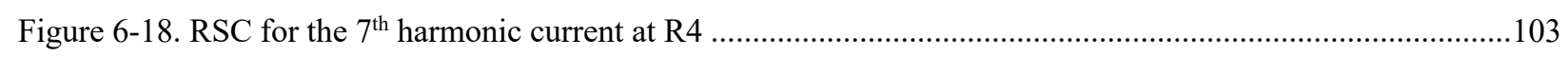

Figure 6-19. $5^{\text {th }}$ harmonic current aggregated phasor values operating in negative RSC at R3 [A] ........................104

Figure 6-20. $7^{\text {th }}$ harmonic current aggregated phasor values operating in negative RSC at R3 [A] .......................104

Figure 6-21. $5^{\text {th }}$ harmonic aggregated phasor values operating in negative RSC at R4 [A] .................................105

Figure $6-22.7^{\text {th }}$ harmonic aggregated phasor values operating in negative RSC at R4 [A] ................................105

Figure 7-1. Location of Site B PQ recorder in high voltage network.................................................................110

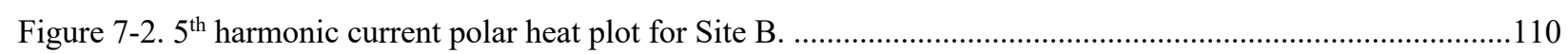

Figure 7-3. Aggregation time interval plots for Site A (R3), all data ...........................................................111

Figure 7-4. Aggregation time interval plots for Site A (R3), one day's data ......................................................112

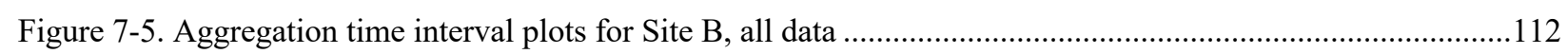

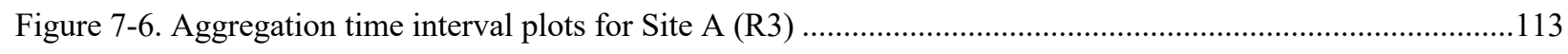

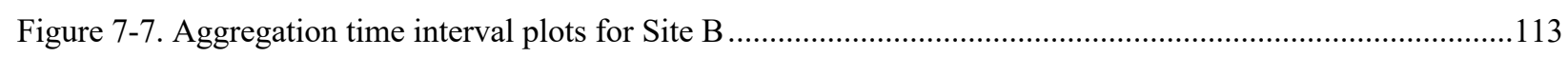

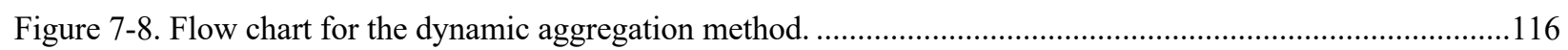


Figure 7-9. Aggregation levels to be used in the dynamic aggregation method. .116

Figure 7-10. Normal aggregation of the $5^{\text {th }}$ harmonic current phase angle .........................................................117

Figure 7-11. Dynamic aggregation for 0.9 level of prevalence ..........................................................................118

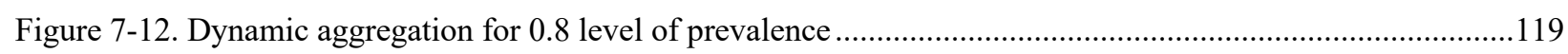

Figure 7-13. Dynamic aggregation for 0.6 level of prevalence .............................................................................119 


\section{LIST OF PEER-REVIEWED CONFERENCE PUBLICATIONS}

1. B. Peterson, A. M. Blanco, J. Rens, J. Meyer, G. Botha, and J. Desmet, "Impact of Aggregation Interval on Harmonic Phase Angle Measurements," in 2018 IEEE 9th International Workshop on Applied Measurements for Power Systems (AMPS), 2018, pp. $1-6$.

2. B. Peterson, J. Rens, and J. Desmet, "Harmonic Emission Assessment on a Distribution Network: the Opportunity for the Prevailing Angle in Harmonic Phasors," in CIRED 24th International Conference on Electricity Distribution, 2017, June.

3. B. Peterson, J. Rens, J. Meyer, G. Botha, and J. Desmet, "On the assessment of harmonic emission in distribution networks: Opportunity for the prevailing harmonic phase angle," in 2016 IEEE International Workshop on Applied Measurements for Power Systems, AMPS 2016 - Proceedings.

4. B. Peterson, J. Rens, G. Botha, and J. Desmet, "A discriminative approach to harmonic emission assessment," in IEEE International Workshop on Applied Measurements for Power Systems (AMPS), 2015.

5. B. Peterson, J. Rens, U. Minnaar, G. Botha, and J. Desmet, "A South African Review of Harmonic Emission Level Assessment As Per IEC61000-3-6," in CIGRE, 2015, pp. 1-9.

6. B. Peterson, J. Rens, J. Meyer, G. Botha, and J. Desmet, "Evaluation of Harmonic Distortion from Multiple Renewable Sources at a Distribution Substation," in IEEE International Workshop on Applied Measurements for Power Systems (AMPS) Proceedings, 2017, pp. 37-42.

7. B. Peterson, J. Rens, G. Botha, and U. Minnaar, "Harmonic emission assessment in a dynamic environment using aggregated harmonic phasors," in CIGRE, 2019. October, pp. $1-11$.

8. Z. Marais, J. Rens, B. Peterson, and G. Botha, "Measurement Uncertainty of Current Harmonics During the Assessment of Compliance to Grid Code Requirements," in 2018 IEEE $9^{\text {th }}$ International Workshop on Applied Measurements for Power Systems (AMPS), 2018, pp. 1-6. 


\section{LIST OF PEER-REVIEWED JOURNAL PUBLICATIONS}

1. B. B. Peterson, J. Rens, M. G. Botha, and J. Desmet, "On Harmonic Emission Assessment : A Discriminative Approach,” Africa Res. J., vol. 108. December, pp. 165-173, 2017.

2. B. Peterson, J. Rens, and J. Desmet, "Harmonic emission assessment on a distribution network: the opportunity for the prevailing angle in harmonic phasors," CIRED - Open access proceedings journal - IET, vol. 2017, no. 1, p. 4, 2017.

3. U. Minnaar, B. Peterson, H. Mostert, J. Rens, and G. Botha, "Power Quality Grid Code Compliance for Renewable Power Plants in South Africa," IET Gener. Transm. Distrib., Nov. 2018. 


\section{CHAPTER 1: INTRODUCTION}

Large-scale renewable energy sources are being rolled out across the world as countries reduce their reliance on fossil fuels for the generation of electrical energy. Following this trend, the South African government opened its networks for the introduction of renewable energy as part of their electrical energy generation mix [2]. A bidding process ensued to ensure competitive pricing amongst the various independent Renewable Power Plants (RPPs ${ }^{2}$ ) wanting to connect onto the South African electrical network.

Due to the details of the bidding process implemented, each RPP must be treated the same, irrespective of the size of the power plant or the technology which is deployed for the generation of electrical energy. The South African grid code on renewable energy [1] was created to ensure that all successful bidders would be treated the same and that they all complied to the same technical criteria. Industry and electrical utilities jointly created and compiled the grid code document to ensure a balanced approach to the technical requirements and it was promulgated by the National Energy Regulator of South Africa (NERSA) [3].

Power Quality (PQ) is one part of the technical requirements specified in the grid code document. While the allocation of PQ emission limits to a Renewable Power Plant (RPP) are easily achieved ${ }^{3}$ by application of the IEC 61000-3-6 [4] principles, the complexity is in proving compliance once the renewable energy plant is operational.

Harmonic voltage distortion is one of the key concerns, therefore harmonic emission from an RPP is of specific interest [5], [6]. Voltage waveform distortion at any Point of Common Coupling (PCC) in an interconnected network is the result of all sources of harmonic currents connected to that network. Although large electro-mechanical generators do generate near-perfect sinusoidal voltage waveforms, non-linear loads and generators do not. At the PCC where the RPP connects, the voltage are not perfectly sinusoidal anymore.

It is the responsibility of the electrical utility to manage the voltage waveform distortion and therefore harmonic emission limits apply to the different users of the network; legally contracted based on the grid code documents. Although RPPs are the focus of this research, many consumers nowadays also use power electronics and due to the inherent nonlinearity, inject harmonic currents when consuming fundamental frequency current. Therefore, the principles investigated in this thesis may be applied to any generator or customer.

Both consumers and generators of electrical energy could contribute to voltage waveform distortion. A nonlinear interaction between the source of harmonic currents and the supply system will occur and determine the extent of the steady-state voltage waveform distortion (voltage harmonic distortion). Contributing factors include the harmonic impedance of the network into which the harmonic currents are injected and the magnitude and phase angle of those harmonic currents.

Harmonic rms current limits for installations in South Africa are derived from the IEC/TR 610003-6 [4] technical report, being the reference document and adapted for local requirements in NRS 048-4 [7]. The phase angle of harmonic currents is not taken into account during these calculations. Apportioning harmonic emission to different users of the electrical network aims to keep the voltage waveform distortion at the PCC low enough to only occasionally ( $5 \%$ of the time) exceed the compatibility levels of the PQ standard in use.

\footnotetext{
${ }^{2}$ In the context of this research, an RPP is referred to as the entire plant up to the Point of Connection to the utility network.

${ }^{3}$ Studies have shown that the IEC 61000-3-6 harmonic emission allocations [109] may not be fair due to different background harmonic levels in networks vs generic harmonic planning levels. The scope of the allocation challenges are beyond the scope of this thesis
} 
Due to the legal aspects of a grid code document, compliance is required and if not achieved, it has to be mitigated [1], for example by the installation of harmonic filters. By October 2016, 102 RPPs were commissioned in South Africa with the capability of generating $6400 \mathrm{MW}$ [2]. If the assessment for compliance to grid code requirements on harmonic emission is affected by the manner in which field data were collected, analysed and the results interpreted, it directly affects the risk of doing business due to the (possibly) unnecessary expenses. Both the National Service Provider (NSP) and the RPP are affected.

\subsection{What is harmonic emission?}

A scientifically sound, yet practical method to assess harmonic emission is needed. The measurement methods, processing techniques, assessment criteria and relevant uncertainty aspects have to be clearly defined for application by both the RPP consultant and utility operator.

For the utility, the impact of nonlinear loads on the network is important. Generally referred to as voltage waveform distortion, it can be qualified and quantified in the frequency domain. Harmonic phasors, when added to the fundamental frequency component, being a perfectly sinusoidal voltage waveform, will result in the voltage becoming non-sinusoidal (a distorted waveform).

Harmonic currents cause harmonic voltage drops across all impedances, distorting the voltage at Points of Common Coupling (PCC).

In this thesis, "harmonic emission" refers to the harmonic currents injected into the network and is the focus of the research, therefore the research question that will be answered in this study is:

How can harmonic current emission be assessed at a single source of harmonic currents to enable the electrical utility to manage contractual agreements on harmonic emission?

\subsection{Direction of harmonic emission}

The direction of harmonic emission is in many cases a bone of contention between utilities and customers [8]-[12], with the one blaming the other for the harmonic distortion that exists at the PCC.

Several methods, such as the Harmonic Vector Method [13], RLC method [14], and the Superposition [13] method, are proposed in literature to assess harmonic emission [15] from nonlinear loads. Harmonic active power is often used in these methods [16], [17]. When only one source of harmonic active power exists, then the harmonic active power will be opposite in sign to the active power transferred by the fundamental frequency component [16]. With a single-point measurement and only one source of harmonic active power, it is possible to use this sign of harmonic active power to locate and assess the harmonic emission.

A real power system always contains more than one source of harmonic active power and these sources are normally distributed all over an interconnected network. These sources of harmonic active power are nonlinear, and the sign of the harmonic active power is no longer absolute using single-point measurements as an exchange of harmonic active power between different sources occur.

The extent of the harmonic power exchange is set by, for example, the difference in firing angle between two thyristor rectifiers in two different nonlinear loads [18]. A source of harmonic active power can be absorbing harmonic active power at a later stage, without any changes in the operating conditions of the electrical equipment. The switch from harmonic source to harmonic sink is due to changes not under the control of that customer, for example, upstream network configuration and another customer changing operating conditions (the firing angle of the thyristor rectifier example). 
A single-point measurement, for this reason, cannot further any useful information on the harmonic emission in a real-life power system [16]. Synchronised measurements at all sources of distortion are needed to understand the harmonic emission and this will result in an extremely complex measurement problem and analysis of data.

Engineers responsible to operate power systems, require a pragmatic solution to quantify harmonic emission. Constraints are set by fundamental network theory. The contribution that this thesis makes is to improve the deficiencies of standardized methods, i.e. the CIGRE/CIRED joint WG C4.109 assessment of harmonic emission that has been prescribed by the South Africa grid code [1].

The CIGRE/CIRED joint working group (WG) C4.109 developed a statistical approach to the qualitative assessment of harmonic emission [19] where 10-min rms values for harmonic voltage and current are used to evaluate harmonic distortion at a PCC. It cannot quantify the exact contribution of the load(s) or source(s) connected to the PCC, as it is a qualitative assessment only and not an absolute assessment of harmonic emission as required by the legal aspects of the grid code document [20].

Network harmonic impedance is needed to apply the CIGRE C4.109 method and from the $95^{\text {th }}$ percentile of harmonic current (rms value), harmonic voltage emission is estimated. Deficiencies of this approach are:

- Interpretational guidelines are not exhaustive.

- By making use of harmonic phasor rms values, it cannot discriminate between harmonic components emitted and those being sunk by a nonlinear load [20].

Furthermore, network conditions and RPP generation change over time. Consequently, harmonic emission continuously changes. This is a very important legal consideration. Results obtained today could be different later on. Harmonic emission requires continuous assessment, as is evident by the establishment of a CIGRE working group C4.42 dealing with continuous assessment of harmonic emission assessment of low-order harmonics.

It is evident that the existing approach to harmonic emission assessment within the context of grid code compliance is deficient. An RPP cannot be held legally liable when declared in breach of grid code agreements by the utility using the existing approach.

\subsection{Research hypothesis}

Harmonic emission assessment for Renewable Power Plants (RPPs) as part of a grid code compliance process requires engineers to use field data obtained from instruments that are certified as compliant to the Class A PQ measurement requirements of IEC 61000-4-30, edition 3. This latest edition included specifications for current measurements (previously only voltage) and improved time-stamping uncertainty.

Deficiencies in existing standardized harmonic emission assessment methods are identified by means of computer modelling and simulation and results validated using field data. An improved solution is then developed to realise the research hypothesis:

Grid code compliance requirements on harmonic emission can be assessed in a fair and pragmatic manner by means of an improved method recognising that:

- A nonlinear and dynamic interaction between the supply network and the grid-connected RPPs results in harmonic currents being injected or absorbed by either.

- The assessment of harmonic emission has to identify those harmonics being injected into 
the supply network and has to exclude those being absorbed by the RPP.

- Harmonic emission assessment has to be done continuously.

Research results reported in this thesis address the above research hypothesis by:

1. Analysis and modelling of harmonic emission from first principles in an interconnected network with more than one source of harmonic waveform distortion being reported.

2. Deriving the metrological requirements and constraints of measuring both voltage and current harmonics.

3. Can the relative contribution to harmonic distortion be quantified when based on rms harmonic measurements obtained from the IEC61000-4-30 Class A, edition $3^{4}$ compliant instrumentation?

4. Can existing harmonic assessment techniques be improved to sufficiently ${ }^{5}$ discriminate between harmonic emission contributed by different RPPs or by a specific distorting load, or is a completely new approach required?

The content and structure of the thesis are presented next.

\subsection{PhD model: A series of scientific publications}

$\mathrm{PhD}$ research is normally evaluated based on a comprehensive thesis that states and motivates the lack of knowledge on a certain topic, then it derives a research hypothesis that addresses that lack of knowledge to which a solution is then developed and presented as a new contribution improving the current field of knowledge.

In engineering research, it can be expected that some mathematical modelling, hard- or software simulation and field testing can be used to validate this contribution to the existing field of knowledge. The approach in this PhD research made use of regular international appraisal of the progress by presenting selected topics for peer review at specialist conferences and accredited scientific journals.

Continuous peer review not only assessed scientific relevance but also the context, as an important goal of the research was to improve the engineering application of harmonic emission assessment. This resulted in the following outputs:

1. B. Peterson, A. M. Blanco, J. Rens, J. Meyer, G. Botha, and J. Desmet, "Impact of Aggregation Interval on Harmonic Phase Angle Measurements," in 2018 IEEE 9th International Workshop on Applied Measurements for Power Systems (AMPS), 2018, pp. $1-6$.

2. B. B. Peterson, J. Rens, M. G. Botha, and J. Desmet, "On Harmonic Emission Assessment : A Discriminative Approach,” Africa Res. J., vol. 108, December, pp. 165-173, 2017.

3. B. Peterson, J. Rens, and J. Desmet, "Harmonic Emission Assessment on a Distribution Network: the Opportunity for the Prevailing Angle in Harmonic Phasors," in CIRED 24th International Conference on Electricity Distribution, 2017, June.

4. B. Peterson, J. Rens, J. Meyer, G. Botha, and J. Desmet, "On the assessment of harmonic emission in distribution networks: Opportunity for the prevailing harmonic phase angle,"

\footnotetext{
${ }^{4}$ Edition 3 is the PQ measuring standard applicable to this work.

5 "Sufficiently" is qualified later on reflecting how well the measurand compares to the measurement uncertainty.
} 
in 2016 IEEE International Workshop on Applied Measurements for Power Systems, AMPS 2016 - Proceedings.

5. B. Peterson, J. Rens, G. Botha, and J. Desmet, "A discriminative approach to harmonic emission assessment," in IEEE International Workshop on Applied Measurements for Power Systems (AMPS).

6. B. Peterson, J. Rens, and J. Desmet, "Harmonic emission assessment on a distribution network: the opportunity for the prevailing angle in harmonic phasors," CIRED - Open access proceedings journal - IET, vol. 2017, no. 1, p. 4, 2017.

7. B. Peterson, J. Rens, U. Minnaar, G. Botha, and J. Desmet, "A South African Review of Harmonic Emission Level Assessment As Per IEC61000-3-6," in CIGRE, 2015, pp. 1-9.

8. B. Peterson, J. Rens, J. Meyer, G. Botha, and J. Desmet, "Evaluation of Harmonic Distortion from Multiple Renewable Sources at a Distribution Substation," in IEEE International Workshop on Applied Measurements for Power Systems (AMPS) Proceedings, 2017, pp. 37-42.

9. B. Peterson, J. Rens, G. Botha, and U. Minnaar, "Harmonic emission assessment in a dynamic environment using aggregated harmonic phasors," in CIGRE, 2019. October, pp. $1-11$.

The following peer-reviewed papers were co-authored in support of this research:

1. U. Minnaar, B. Peterson, H. Mostert, J. Rens, and G. Botha, "Power Quality Grid Code Compliance for Renewable Power Plants in South Africa," IET Gener. Transm. Distrib., Nov. 2018.

This paper addressed the development of grid code compliance processes and requirements in South Africa. The $\mathrm{PhD}$ candidate's contributions are reported in section 2, 4, 5, 6 and 8 .

2. Z. Marais, J. Rens, B. Peterson, and G. Botha, "Measurement Uncertainty of Current Harmonics During the Assessment of Compliance to Grid Code Requirements," in 2018 IEEE $9^{\text {th }}$ International Workshop on Applied Measurements for Power Systems (AMPS), 2018, pp. 1-6.

This paper analysed uncertainty in measurement during grid code compliance assessment, using case studies from Southern Africa. The PhD candidate's specific contributions are reported in section II.

\subsection{Thesis structure}

The structure of the thesis expands on the publications above by presenting context and additional detail needed to develop a concise storyline for the purpose of a well-rounded integrated scientific research thesis.

Collectively, these papers contribute to existing knowledge on harmonic emission assessment by presenting an alternative and improved approach.

Chapter 2 analyses harmonic emission assessment methodologies, using fundamental network principles and critically reviews scientific publications to identify the deficiencies in existing approaches to harmonic emission assessment.

Chapter 3 verifies the harmonic emission methodologies discussed in Chapter 2 by applying each method to the same simple circuit consisting of a Thévenin and Norton equivalent circuit under controlled conditions. 
Chapter 4 analyses the standardised approach of the CIGRE/CIRED C4.109 statistical method and then by field data, demonstrate the constraints in harmonic emission assessment. It is shown that using only rms values of harmonic phasors, due to the aggregation interval (10-min) and using single-point measurements, that the objectives of a grid code agreement on harmonic pollution cannot be realised.

Chapter 5 evaluates the application of harmonic phasors in harmonic emission assessment and then by using field data at a PV plant integrated into a medium voltage (MV) distribution network, demonstrates how the data volume becomes impractical when collecting harmonic phasors when measured by the 10/12-cycles (200 ms) specified by IEC61000-4-30 Class A, edition 3 PQ measuring standard. Aggregation of harmonic phasors are proposed as the IEC61000-4-30 data reduction (aggregated values resulting) does not retain harmonic phase angles.

It is concluded in Chapter 5 that aggregated harmonic phasors can improve the results obtainable by the CIGRE/CIRED C4.109 method.

Chapter 6 investigates whether the methodology of Chapter 5 can be extended to a high voltage (HV) network when multiple harmonic sources are connected to the same PCC. The dynamic character of harmonic emission is demonstrated and how synchronised measurements can, in addition to aggregated harmonic phasors, improve harmonic emission assessment.

Chapter 7 addresses a shortcoming of the proposed solution to harmonic emission assessment when using aggregated harmonic phasors. During the initial research, the aggregation interval was fixed for the whole dataset and due to the dynamic character of nonlinear loads and network configuration, it was realised that the time aggregation interval has to be dynamically adjusted.

Chapter 8 concludes by an integrated summary of how the findings of all publications addressed the research hypothesis. 


\section{CHAPTER 2: Harmonic Emission: What is currently known}

\subsection{Introduction}

In this Chapter, a critical analysis of the fundamental principles of harmonic emission and known (accredited) methods to assess it, are presented. Methods of harmonic emission assessment that seem to be useful for electrical utilities in assessing harmonic emission, are discussed.

Large-scale Renewable Power Plants (RPPs) are investigated as sources of harmonic distortion, because during the time of the research, RPPs were a fast growing and a new source of voltage waveform distortion in Southern Africa. Electrical utilities have to monitor their contribution to the overall PQ pollution and with RPPs using the distribution network by contracted agreements, compliance to this agreement requires field data to be used in assessing how much harmonic emission they contribute.

In the next Chapter, the harmonic emission assessment methods discussed are applied to simplified circuits by means of simulations to further understand the dynamics of each method under controlled conditions.

\subsection{What is harmonic emission?}

The IEC 61000-3-6 technical report defines emission as a "phenomenon by which electromagnetic energy emanates from a source of electromagnetic disturbance" [4]. The document further defines a harmonic emission level $\left(\boldsymbol{U}_{h i}\right)$ as a harmonic phasor at a particular harmonic order as illustrated in Figure 2-1 (it is acknowledged that the phasor diagram may vary depending on any mitigation equipment installed, load types and network topologies).

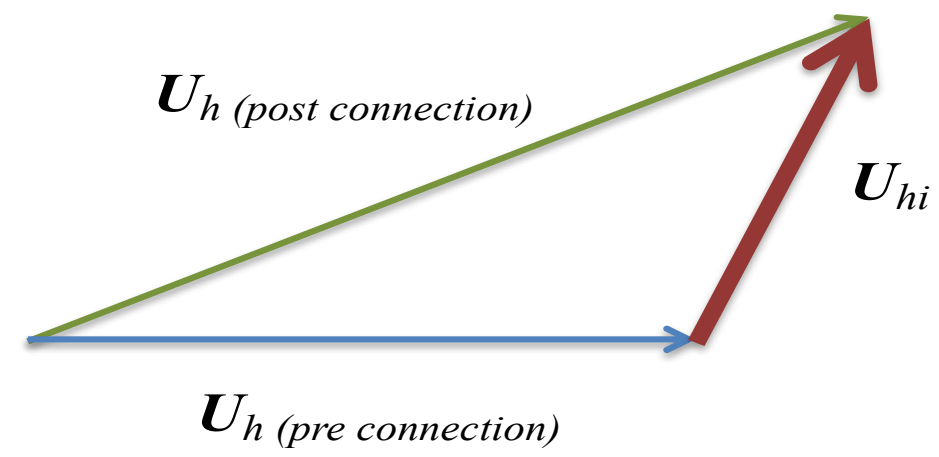

Figure 2-1. Illustration of voltage harmonic emission phasor $U_{h i}$ [4]

The IEC 61000-3-6 approach recognizes that once a distorting load has been connected to a node

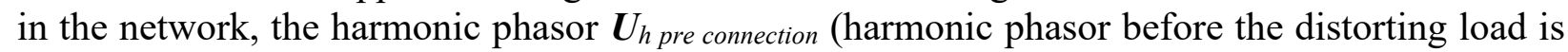

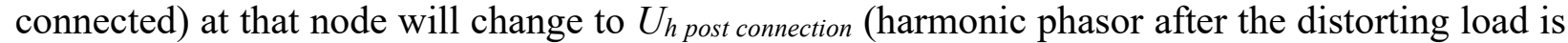
connected) due to emission contributed by the distorting load, where the emission phasor is designated as $\boldsymbol{U}_{h i}$ (note that $i$ is not defined in the IEC 61000-3-6 document, it is assumed to refer to the non-linear load).

Per definition, for single load, the resulting voltage harmonic emission is the difference between the voltage harmonic phasor before the load is connected and the voltage harmonic phasor after the load is connected. 


\subsection{Renewable energy sources and voltage harmonic distortion}

Usage of renewable energy sources are on the increase across the world as it is seen as a clean source of energy from an environmental viewpoint in comparison to fossil fuels [21]. These renewable energy sources are a cause of PQ concerns in the electrical networks to which they connect [22]-[25]. Renewable energy sources such as PV and new generation wind turbines, make use of solid-state power electronics to convert renewable energy to electrical energy. This power electronic conversion process is nonlinear in principle and in addition to the $50 \mathrm{~Hz}$ (fundamental frequency) current, harmonic currents are also injected. Harmonic currents cause harmonic voltages across the system impedances, distorting the utility voltage waveform.

Integration of RPPs into a Distribution System Operator (DSO) or Transmission System Operator (TSO) network requires the RPP to prove grid code compliance [1], [26]-[30].

Grid code compliance is absolute with no grey areas. As such, the evaluation and determination of grid code compliance must be done fairly and with sound engineering principles.

The next section gives a brief overview of where the harmonic distortion emanates from the RPPs, with a focus on wind and solar renewable energy.

\subsubsection{Harmonics from wind turbines}

Wind energy interfaces with the electrical grid by means of four different types of wind turbines. The type A or Type 1 (Figure 2-2) wind turbine was popular during the 1980s and had a fixed speed with a direct grid connection by means of a soft starter [31].

The type B or Type 2 (Figure 2-3) wind turbine was introduced in the mid-1990s allowing some speed control of the turbine [31] by means of a variable rotor resistance and these turbines were also connected directly onto the grid by means of a soft starter.

Type A and Type B wind turbines generate minimal ${ }^{6}$ harmonics as they do not employ any power electronic circuits in the power generation path.

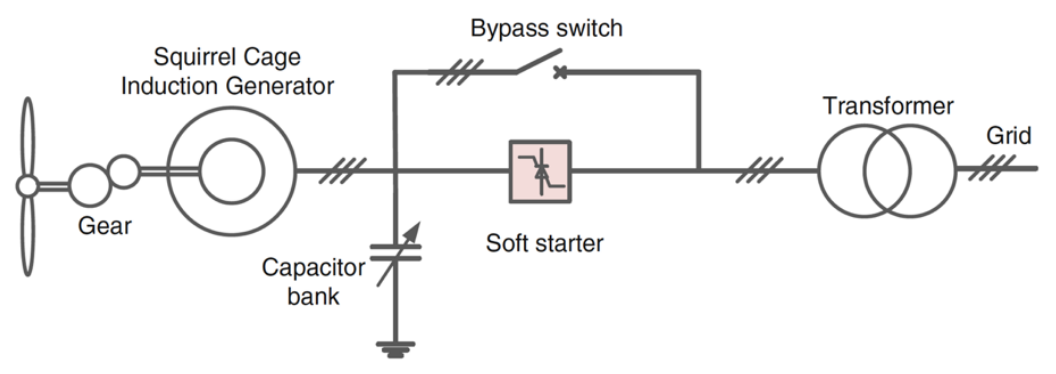

Figure 2-2. Fixed wind speed Turbine-Type A or Type 1 [31]

\footnotetext{
${ }^{6}$ The harmonics generated by an induction generator is dependent on the physical construction of the windings; it is however minimal and generally regarded as a clean source.
} 


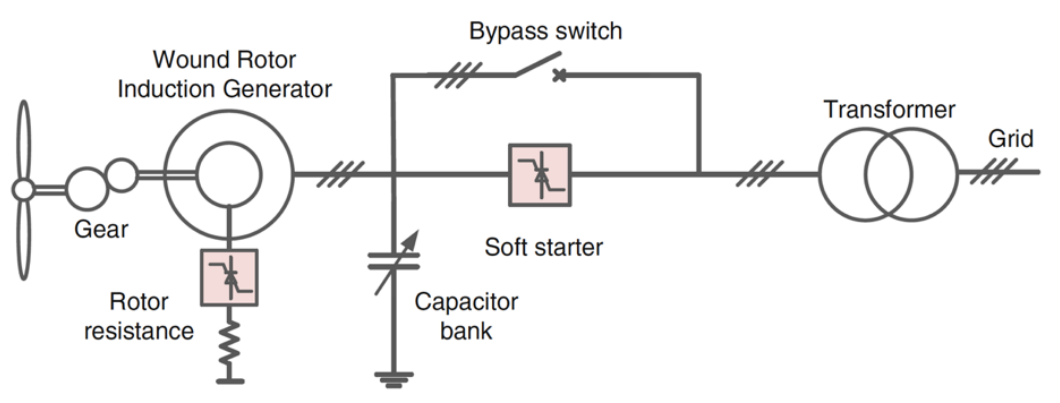

Figure 2-3. Partial variable speed wind turbine - Type B or Type 2 [31]

Type C or Type 3 (Figure 2-4) wind turbines have been in existence since the early 2000s and they are the most established technology in service. A squirrel cage induction machine to a doubly fed induction machine with a back-to-back power electronic converter to control the speed of the machine. The power converter can be used to regulate the output power of the machine.

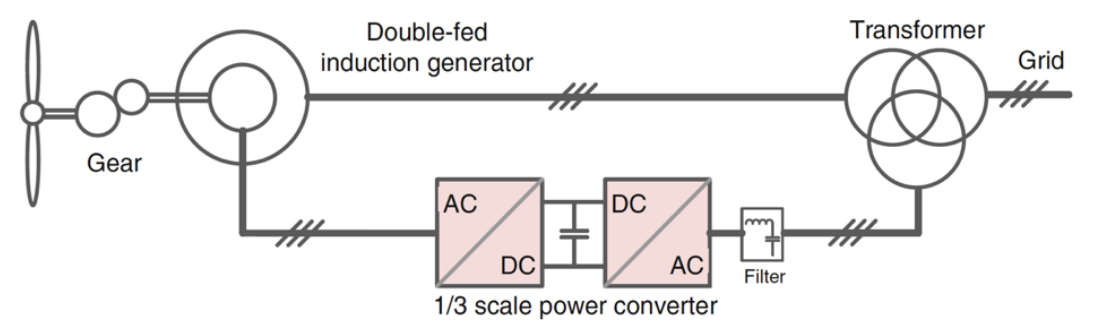

Figure 2-4. Variable speed wind turbine with partial power converter - Type C or Type 4 [31]

Type D or Type 4 wind turbines are either asynchronous generators, wound rotor synchronous generators or permanent magnet synchronous machines that are connected to the grid via fully rated converters, i.e. all the energy is transferred to the grid via the converter. [31].

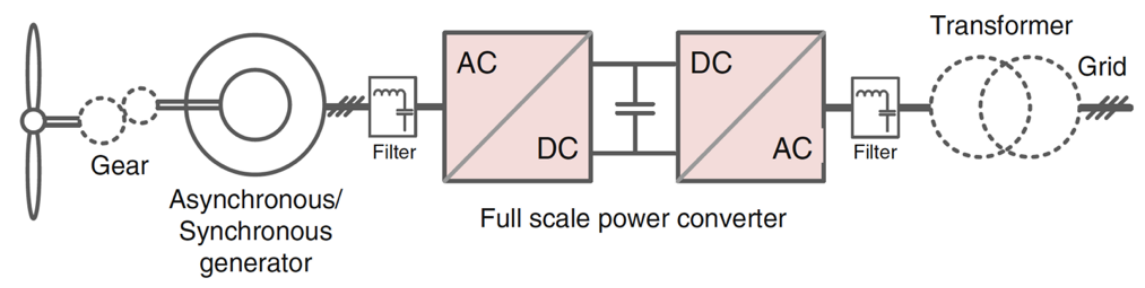

Figure 2-5. Variable speed wind turbine with Full power converter - Type D or Type 4 [31]

Type $\mathrm{C}$ and Type $\mathrm{D}$ wind turbines use power electronics causing voltage harmonic distortion and harmonic filters are used to contain it. This cannot be done perfectly and wind farms do have an effect on voltage harmonic distortion [32], [33], [34] in the grid they are connected to. Type D wind turbines, as a result of the improved control by power electronics, have the most potential to increase harmonic distortion levels in the connecting grid [31] .

It was found [35] that offshore wind farms have a significant potential for harmonic resonant amplification due to the capacitance of the high voltage cables used to connect to the land-based transmission network. 


\subsubsection{Harmonics from photovoltaics}

Photovoltaics (PV) installations convert solar energy into electrical energy by the typical configuration shown in Figure 2-6 [22].

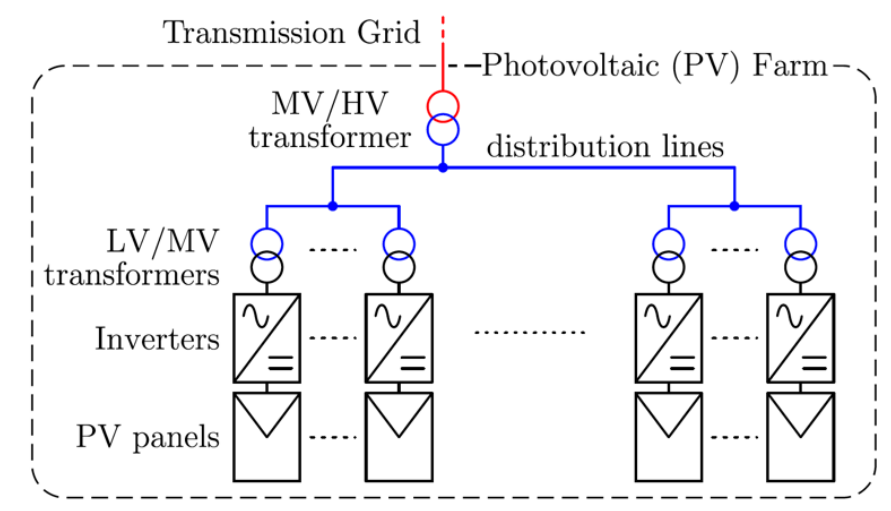

Figure 2-6. Simplified PV plant architecture [36]

Manufacturers of inverters are required to characterise the harmonic output of inverters. Too high levels of harmonic current injection have to be avoided by grid operators. These tests, when done under ideal conditions where harmonic distortion at the PCC is low, will be different when installed in a real network with higher levels of background voltage harmonic distortion [37]-[40]. Acceptable levels of harmonic distortion at a PCC (utility level) are defined in documents such as EN50160 [41] and NRS048 [7].

Studies [42] found that an IEEE 1547 [40] compliant inverter had a current Total Demand Distortion (TDD) value of $0.66 \%$ when no harmonic voltage distortion exists in the emulated grid voltage waveform, but increase to a current TDD of more than $20 \%$ when only a $3 \%$ voltage harmonic distortion was emulated. A 20\% increase in current TDD output from inverters is a real concern for DSOs and TSOs, as the cumulative effect of the increase in current TDD may increase the background harmonic voltage distortion levels through the network's harmonic impedance.

In [43] it was found that the harmonic current THD output of an inverter is inversely proportional to the output power, as shown in Figure 2-7. It also indicated that the high current THD was dominated by low-order harmonic emissions at low power output levels and as power output increased, low-order harmonic values decreased. The risk of exceeding harmonic emission levels at a PV plant is the greatest at sunrise and sunset [44]-[46]. 


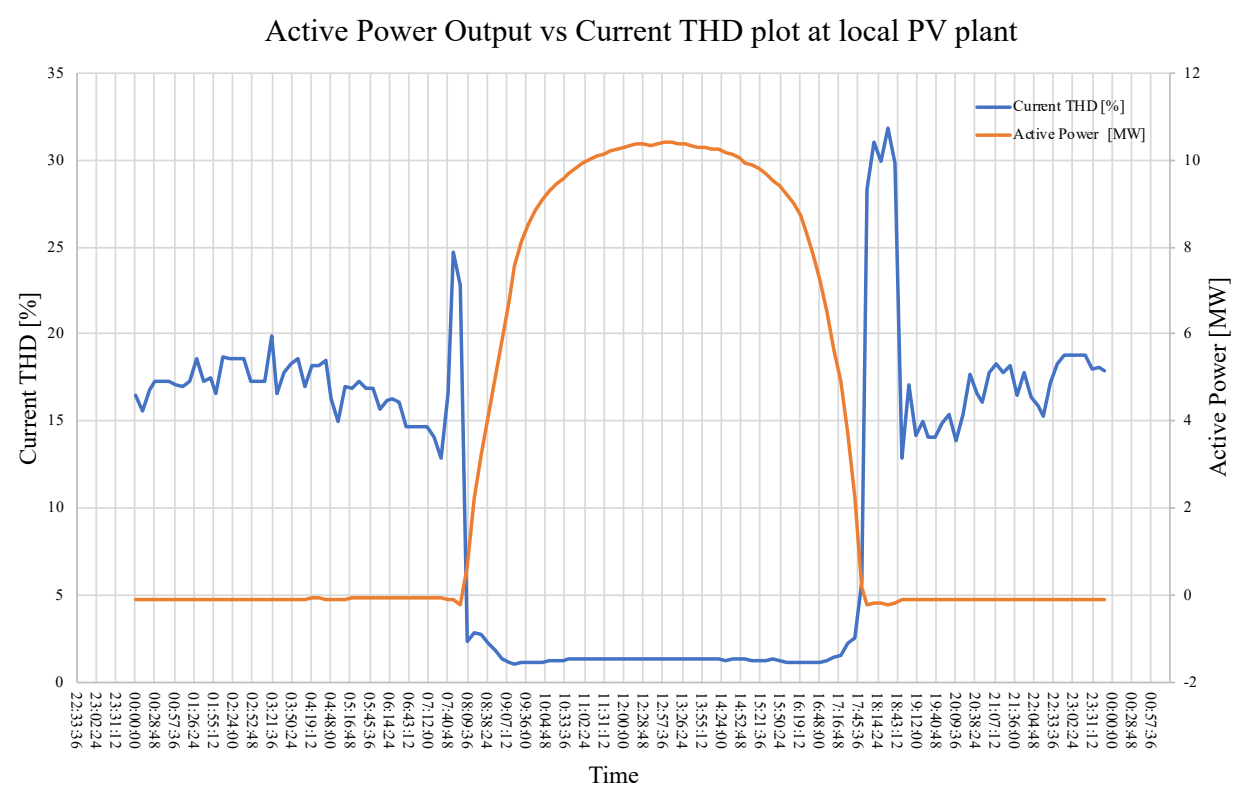

Figure 2-7. Current THD against Power output of a PV inverter at a local PV plant [43] over a 24 hour period

\subsection{Why harmonics are a concern for electrical utilities}

Electrical utilities have to contain levels of harmonic distortion to acceptable norms, as defined by technical standards on voltage quality such as EN 50160 [41] and NRS048 [7]. Voltage and current harmonics can reduce the life expectancy of electrical equipment [47], [17] with transformers and motors most susceptible.

As the electrical network transforms from a centralized electrical grid system (centralized generation power stations) to a distributed electrical system (distributed renewable energy sources), the safe integration of renewables power sources that generate harmonic pollution [31] to the network must be managed.

It is important to maintain electromagnetic compatibility between sensitive electrical and electronic components to assure uninterrupted and high-quality supply of electricity on the network.

In order to preserve good quality electricity on the network, regulatory standards are available that specify the limits that must be imposed on loads and networks, with the objective of providing an environment in which Electromagnetic Compatibility (EMC) is achieved [7], [41]. To ensure the compatibility of equipment connected on the network, it is necessary to control the maximum level of disturbance that may be present at any point in the network and establish a level of disturbance to which every piece of equipment will be immune. In achieving EMC, it is stated in [7] that several parameters are to be specified and controlled. These are:

\begin{tabular}{ll}
- & Emission level; \\
- & Immunity level; \\
- & Compatibility level; \\
- & Emission limit; \\
- & Immunity limit; \\
\hline & Planning level.
\end{tabular}

In South Africa, the NRS048 specification is the application practice that provides guidelines to power utilities in the management of low-frequency EMC parameters. Part 2 of the specification represents utilities' internal objectives in designing the network in order to ensure system 
compatibility [44.] Figure 2-8 shows the inter-relationship of these low-frequency EMC parameters.

Illustrated in Figure 2-8 is the way in which compatibility between the voltage waveform and the electrical equipment connected to this voltage should be managed from a general PQ perspective. A probability density of the components is required to manage PQ (in this thesis harmonic distortion is the main focus) in order to maintain a balance between network performance and equipment susceptibility levels.

The system disturbance performance level comprises the background voltage THD levels (from adjacent networks) as well as total harmonic distortion emissions generated by other nonlinear loads connected to the same PCC. The equipment immunity level is the PQ level at which the equipment will operate within its design limits and not be negatively impacted. Electrical utilities are expected to maintain the system disturbance levels to below the respective PQ limits for 95\% of the time, as indicated by documents such as EN 50160 [41] and NRS048 [7]. Similarly, the equipment used on these electrical networks must be able to "ride" (maintain functionality) through network disturbances for $95 \%$ of the time. Equipment immunity or the equipment's ability to "ride" through disturbances will vary amongst different manufacturers.

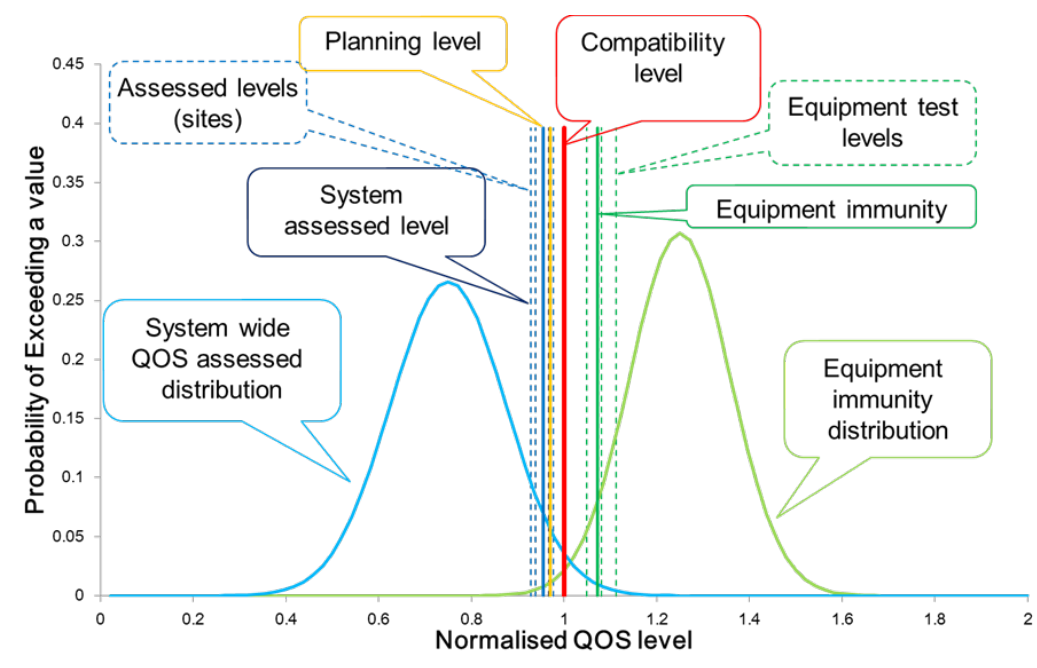

Figure 2-8. Illustration of basic Power Quality management adapted from [4]

Electrical utilities manage voltage THD contributed by distorting customers at a Point of Connection (PCC) by apportioning emission [8] values to each user connected.

\subsubsection{Harmonic apportionment}

Allocation of harmonic limits at distorting loads is described in IEC 61000-3-6 [4]. When harmonic currents are less than these limits, then the resulting voltage harmonics when these harmonic currents flow through system impedances, will, in principle, not increase the voltage THD above the compatibility level as discussed in section 2.4 above.

Fundamentally it is about Ohm's law and the impedance of the network. The goal is to not exceed planning levels in voltage harmonics or voltage THD, where the planning levels are lower than the compatibility levels in the applicable PQ standard. Therefore, the planning level is equitably divided between the different loads, based on the power level of these loads relative to the capacity of the network.

Three stages for the calculation of emission limits [4] are defined in IEC61000-3-6: 
- $\quad$ Stage 1 is a simplistic approach in which the size of the load is evaluated against the system short-circuit power. If the ratio of these two factors is less than $0.2 \%$, then the load is allowed to connect to the network without any specific emission limits allocated [38]. If the ratio is higher than $0.2 \%$, then stage 2 is initiated.

- $\quad$ Stage 2 is a more detailed, Point of Connection (PoC) specific approach in which the size of the disturbing load is considered relative to the installed capacity of the network to determine specific emission levels that the load must comply with. Installed capacity (not to be confused with fault level) refers to the network maximum power transfer capability at the $\mathrm{PoC}$.

- If a plant cannot meet the emission levels specified in stage 2, then they may request the DSO or TSO to re-evaluate the allocated emission levels under stage 3. An appraisal of the existing distortion levels at the PoC is undertaken by the system operator to determine if a higher allocation can be allowed. Higher allocations are conditional and allow the plants to defer any cost for mitigation equipment subject to the conditions stipulated by the system operator.

Note that apportioned limits do not consider measurement uncertainty in the equipment used to assess compliance to these limits. Documents such as IEC 61400:21 [48] state that harmonic current emissions below $0.1 \%$ of the rated current of a wind turbine should not be reported as the measurement uncertainty at this level could probably be higher than the measurement. Measurement uncertainty in real-world applications have not been extensively validated and to what extent apportioning takes this into account, is largely unknown.

\subsubsection{Harmonic emission assessment (IEC)}

Benchmarking of PQ against a technical standard mostly makes use of 10-min rms voltage and current parameters resulting from IEC 61000-4-30 [49] aggregation principles. Because harmonic phasors, as resulting from the fundamental 10/12-cycle block of data (IEC 61000-4-30), can significantly change in magnitude and angle between subsequent blocks of data, a summation exponent is used to compensate for the lack of phase angle information when applying the summation law [4] as given in (1).

$$
U_{h}=\sqrt[\alpha]{\sum_{i} U_{h i}^{\alpha}}
$$

Where $U_{h}$ is the magnitude of the aggregated harmonic voltage, $U_{h i}$ the magnitude of the individual harmonic phasors and $\alpha$ the summation exponent listed in Table 2-1.

An $\alpha$ value of 1 means that harmonic values add up linearly (harmonic phasors are perfectly aligned) and an $\alpha$ value of 2 indicates values that add up orthogonally [50], [51]. By using the $\alpha$ exponent, the variation in harmonic phase angles is estimated.

Table 2-1: Summation exponent values

\begin{tabular}{|c|c|}
\hline Harmonic order & $\boldsymbol{\alpha}$ \\
\hline $\mathrm{h}<5$ & 1 \\
\hline $5 \leq \mathrm{h} \leq 10$ & 1.4 \\
\hline $\mathrm{h}>10$ & 2 \\
\hline
\end{tabular}

Assuming that voltage THD at a PoC to a distribution network can be assessed by comparing it to what the voltage THD was before the load was connected, the concept of "pre" and "post" 10-min rms values for the voltage harmonic phasors under investigation can be used in (2) below. 


$$
U_{\text {hi }}=\sqrt[\alpha]{U_{\text {post connection }}^{\alpha}-U_{\text {pre connection }}^{\alpha}}
$$

A harmonic emission assessment, using the IEC general summation law, assumes that the pre- and post-connection data are measured under the exact same network operating conditions as well as over a similar time period. In practice this is not always possible. For example, an RPP required to measure the pre-connection harmonic distortion can record 10-min aggregated rms voltage harmonics at a PoC assumed (typically an existing busbar belonging to the electrical utility) prior to the RPP plant being built. As time progresses and the RPP plant is built, the upstream utility network can change (additional loads and different network configuration) and that can result in different network (background) voltage harmonics.

Another example of pre-connection measurement is when the RPP plant is already in operation and generating onto the utility grid. RPPs will take the no-generation mode (i.e. zero or little energy output) as the pre-connection measurement. The problem with this approach is that the voltage harmonic distortion measured is not purely the background values, the harmonic impedance of the RPP will invariably affect the measured voltage harmonic distortion at this point, because some of the harmonic currents from the upstream network will flow into the RPP's low impedance paths, where they exist.

In cases where the RPP plant is already generating electrical energy onto the utility network, the circuit breaker connecting the RPP to the network must then be opened to ensure that the preconnection measurement is purely due to the upstream network (background) and no influence from the RPP is encountered. RPPs are, however, reluctant to do this as this results in a loss of revenue.

The fixed static summation exponents listed in Table 2-1 may lead to the incorrect assessment of voltage harmonic emission. In a network where a number of nonlinear loads are connected onto the same node, the impact of harmonic interaction between the different sources of harmonic distortion cannot be ignored [16], [52].

A study conducted by [50] has shown that the probability of harmonics summating as suggested in IEC 61000-3-6 is low and that alternative summation exponents have to be used. The challenge is to know which summation exponent to use under which conditions. The dynamic behaviour of harmonics is dependent on the nonlinear load, the respective harmonic impedances of the network as well as other loads connected to the same busbar [53].

\subsection{Comparison of harmonic distortion localisation methods}

In order to evaluate harmonic emission correctly, the direction of the harmonic distortion must be determined. Finding the source of harmonics at a Point of Common Coupling (PCC) is not straight forward [10]-[12], [14], [54]-[57].

Table 2-2 lists a summary of methods claiming to determine the direction of harmonic emission. Each employs a different approach in terms of the number of measurement points, type of parameters and information required to evaluate the direction of the harmonic distortion.

Each method listed in Table 2-2 is analysed below to better understand the scientific validity and to determine the opportunity in which to apply it in an engineering solution supporting the goal of the research described in this thesis. 
Table 2-2 Various methods for harmonic direction determination [15]

\begin{tabular}{|c|c|c|}
\hline Method & Type of Measurement & Required data \\
\hline $\begin{array}{c}\text { Harmonic state estimation (HSE) } \\
\text { [58] }\end{array}$ & Multipoint & $\begin{array}{c}\text { Multipoint synchronous } \\
\text { measurement }\end{array}$ \\
\hline $\begin{array}{c}\text { Global power quality index [59], } \\
\text { [60] }\end{array}$ & Multipoint & $\begin{array}{c}\text { Multipoint synchronous } \\
\text { measurement }\end{array}$ \\
\hline $\begin{array}{c}\text { Active power [61] } \\
\text { Harmonic current vector method } \\
{[13],[62]}\end{array}$ & Single point & $\begin{array}{c}\text { Voltage and current at the } \\
\text { PCC }\end{array}$ \\
\hline RLC [14] & Single point & $\begin{array}{c}\text { Voltage and current at the } \\
\text { PCC and utility impedance } \\
\text { PCC and utility impedance }\end{array}$ \\
\hline $\begin{array}{c}\text { Superposition and projection } \\
\text { method [13] }\end{array}$ & Single point & $\begin{array}{c}\text { Voltage and current at the } \\
\text { PCC, utility and customer } \\
\text { impedance }\end{array}$ \\
\hline
\end{tabular}

In Chapter 3: the harmonic emission assessment methods are further validated by applying each method to the same basic circuit consisting of either a Norton or Thévenin circuit as a network equivalent with a Norton equivalent circuit representing the nonlinear load. The purpose of this validation through simulation is to better understand the methods in a controlled environment and to highlight any constraints that may not be evident in literature.

\subsubsection{Harmonic active power in the localisation of harmonic sources}

This method is based on the flow of harmonic active power relative to the flow of the fundamental $50 \mathrm{~Hz}$ active power and is used to determine the direction of harmonic emission. Harmonic active power for each harmonic order is calculated using (3).

$$
P=V_{P C C h} I_{P C C h} \cos \left(\varphi_{V h}-\varphi_{I h}\right)
$$

Where:

$V_{P C C h}$ : magnitude of the harmonic voltage at the PCC

$I_{P C C h}$ : magnitude of the harmonic current at the PCC

$\varphi_{V h}:$ phase angle of the harmonic voltage at the PCC

$\varphi_{\text {Ih }}$ : phase angle of the harmonic current at the PCC

The results of the harmonic active power method (HAPM) are interpreted as follows [61];

- If the calculated harmonic active power is negative $(\mathrm{P}<0)$, then the load or downstream network is the source of the harmonic distortion. This harmonic active power flow is opposite to the flow of the fundamental active power.

- If the calculated harmonic active power is positive $(\mathrm{P}>0)$, then the upstream network is the source of the harmonic distortion. The flow of harmonic active power is in the same direction as the fundamental active power flow.

Alternatively, the harmonic active power method is assessed by means of calculating the complex harmonic power [17], using the harmonic voltage and current phasors. IEEE 1459-2010 illustrates the concept of the harmonic active power flow by means of a four-quadrant power diagram [63].

The complex power is calculated using (4)

$$
\boldsymbol{S}_{h}=\boldsymbol{V}_{h} * \boldsymbol{I}_{h}
$$


Where:

$\boldsymbol{S}_{h}$ : complex harmonic power,

$V_{h}: \quad$ harmonic voltage phasor,

$\boldsymbol{I}_{h}$ : harmonic current phasor.

In calculating the complex power, the location of the resultant phasor is used to visualize and evaluate whether harmonic active power is being injected into or out of a PCC.

The results of (4) are plotted on a polar plot, where the active power is plotted on the X-axis and the reactive power is plotted on the Y-axis. If the complex harmonic power points are located to the left of the $\mathrm{Y}$-axis (in quadrant 2 and quadrant 3 with reference to Figure 2-9), then $\mathrm{P}<0$, indicating that the source is downstream. If the complex harmonic power points are located on the right (in quadrant 1 and quadrant 4 with reference to Figure 2-9), then $\mathrm{P}>0$, indicating that the source is the upstream network.

Note that the above assessment holds true when one source of harmonic distortion exists in the load connected at the PoC.

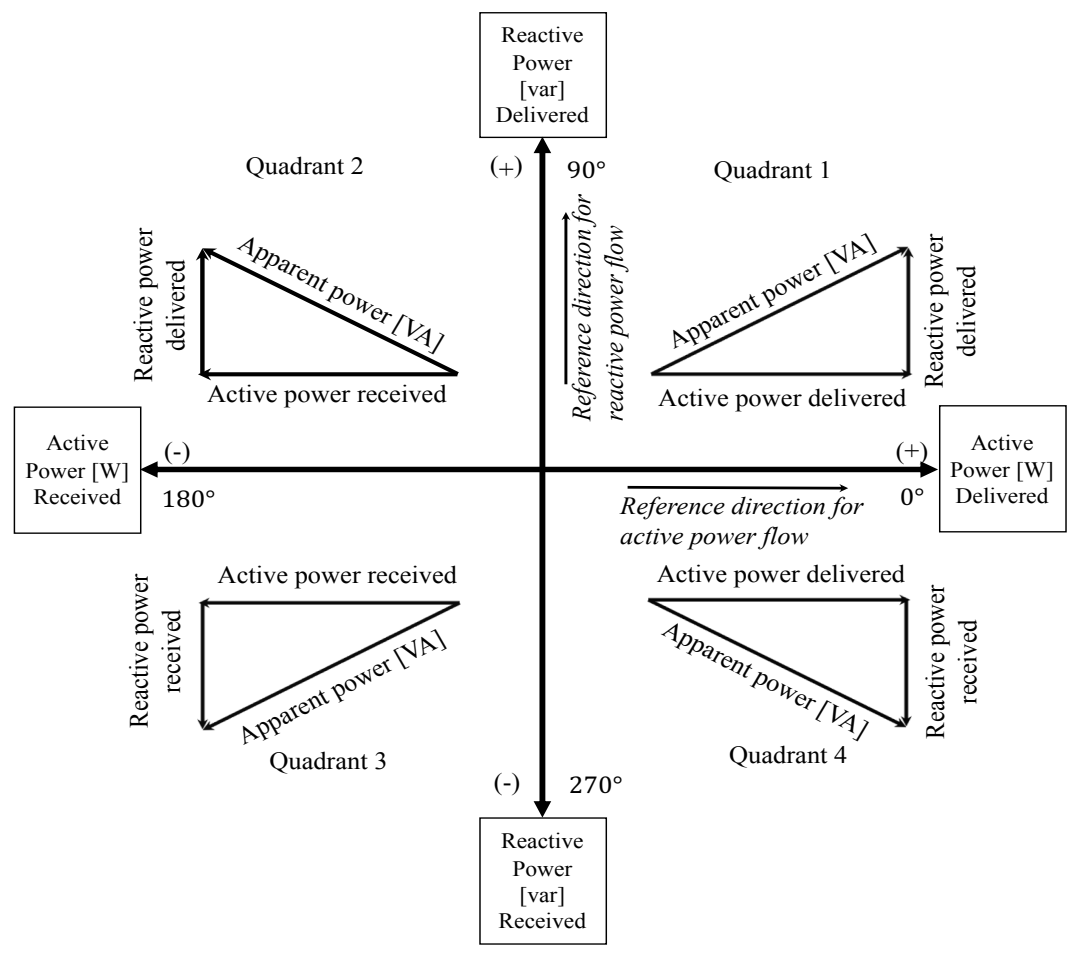

Figure 2-9. Four-quadrant power flow direction (adapted from [63])

It is claimed in [61] that through the mathematical analysis of a simple circuit (shown in Figure 2-10), the HAPM is not always conclusive and should not be used to determine harmonic direction. It was shown that the harmonic active power method does not conclusively indicate the direction of harmonic distortion when the harmonic current phase angles of the load and the upstream network are more than $190^{\circ}$ apart. The study in [61] was reassessed by [64] and found that the HAPM can be used but under certain network conditions, specifically when resonance conditions exist in the supply network resulting in higher harmonic voltages in the supply network.

Harmonic current exchanges between multiple nonlinear loads connected onto the same PCC will result in some of the harmonic currents flowing in opposite directions [52] relative to each other, due to the different operations and make-up of the various nonlinear loads. For this reason, [16] 
concluded that the harmonic active power method is only useful when there is only one source of harmonic distortion in the network.

\subsubsection{Harmonic state estimation}

Harmonic state estimation is based on a mathematical relationship between limited harmonic measurements on a transmission network and system-state variables [58]. The system-state is given by (5) below, a nonlinear ordinary differential equation.

$$
\boldsymbol{x}(t)=f(\boldsymbol{x}, \boldsymbol{u}, t)
$$

Where:

$\boldsymbol{x}: \quad$ state phasor

$\boldsymbol{u}$ : input phasor

$t$ : time variable

The state of the network must be known at $t=0$, as the network changes dynamically and nonlinear loads affects the present network condition. Network component uncertainties, such as transmission line parameters, must be taken into account [65], [66].

Harmonic state estimation provides a broad overview of the harmonic distortion level across the power system. A vast synchronised measurement scheme is required to ensure coherent assessment of the network state. Harmonic phasors are needed, introducing special metrological requirements to contain measurement uncertainty.

Continuous recording of harmonic phasors causes a large volume of data and furthermore network impedances to be known accurately. At transmission level, this is easier due to less substations compared to distribution networks and transmission network configurations, therefore, impedances should be less of a concern.

An online instantaneous assessment of harmonic load flow can be achieved by application of the harmonic state estimation method. Determining specific harmonic emissions from a nonlinear load is not pragmatic when using this method. RPPs are concerned with the harmonic emissions from their own plant, and as such will not be able to install the number of PQ instruments required to implement this method. System Operators (SO) may not grant RPPs access to the data they require for harmonic emission assessment.

\subsubsection{Global power quality}

The global power quality method [59], [60] aims to qualitatively assess which feeder is contributing the most harmonic distortion in terms of THD (Total Harmonic Distortion) to the PCC.

Synchronous measurements are taken on each feeder connected to the PCC of concern. Both the rms voltage and rms current harmonic values are measured and evaluated. Instead of evaluating the individual harmonic distortion levels per feeder, a global approach is taken where the THD is evaluated on a per feeder level. A "global" voltage and current THD factor is defined in (6) and (7) below, respectively.

$$
G T H D_{U}=\sqrt{\frac{U_{\Sigma}^{2}}{U_{\Sigma_{+1}}^{2}}-1}
$$




$$
G T H D_{I}=\sqrt{\frac{I_{\Sigma}^{2}}{I_{\Sigma_{+1}}^{2}}-1}
$$

Where:

$G T H D_{U / I}: \quad$ global THD factor for voltage or current

$U_{\Sigma}: \quad$ rms values of the fundamental frequency components (harmonic) for voltage

$U_{\Sigma+1}$ : $\quad$ positive sequence rms values of the fundamental frequency components (harmonic) for voltage

$I_{\Sigma}: \quad$ rms values of the fundamental frequency components (harmonic) for current

$I_{\Sigma+1}: \quad \quad \quad \quad$ positive sequence rms values of the fundamental frequency components (harmonic) for current

Inclusion of the positive sequence values aids in the determination of the direction of the global harmonic distortion from a non-linear load, by dividing (7) and (6) to result in index $\eta$ [68], [69]. Further analysis is required when low harmonic distortion exist as the value of $\eta$ may be negative. To overcome this constraint $\eta$ is squared $\left(\eta^{2+}\right)$ with the results interpreted as follows.

- If $\left(\eta^{2+}\right)=1$ then the load is balanced

- If $\left(\eta^{2+}\right)>1$ then distortion is injected by the load

- If $\left(\eta^{2+}\right)<1$ then distortion is injected by the source

THD is a ratio of the root mean square (rms) value for the harmonic content of the line voltage to the rms value of the fundamental voltage [67]. The global PQ method does not consider the constitution of the THD value in terms of the contributions from individual harmonic orders, rendering this method ineffective for grid code compliance.

At a utility scale, this method may bear fruit as the utility may be interested in the global management of PQ performance and only track the THD and not the individual harmonics.

\subsubsection{Harmonic Vector Method (HVM)}

The Harmonic Vector Method (HVM) represents the utility network and customer network by Norton equivalent circuits as illustrated in Figure 2-10.

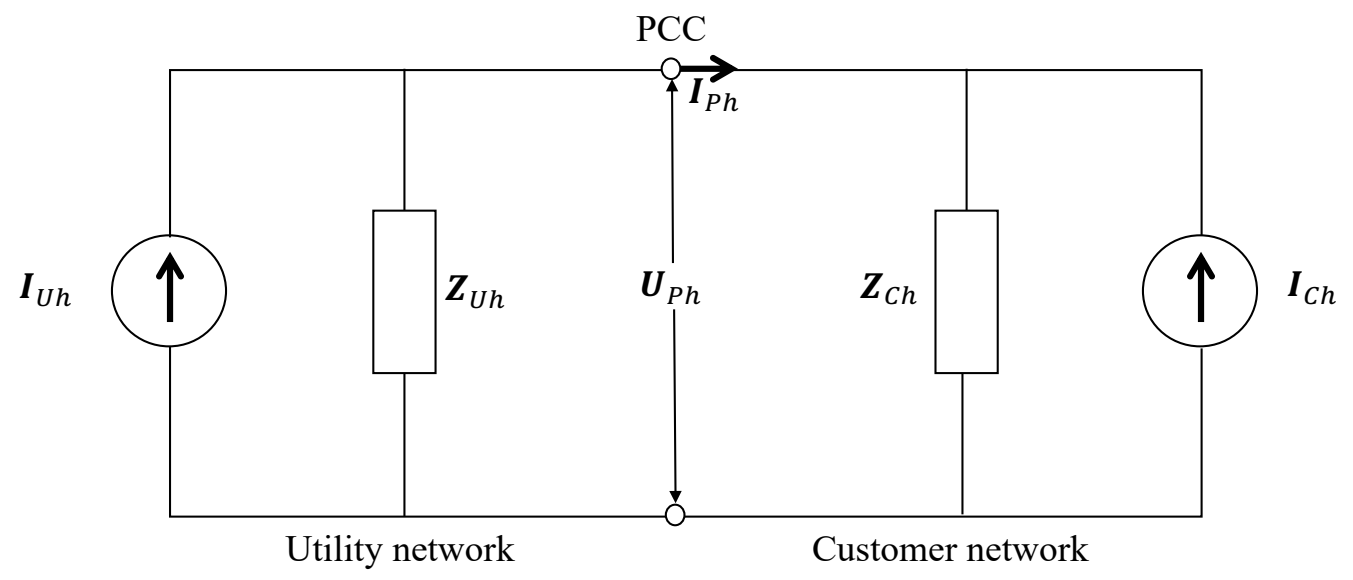

Figure 2-10. Norton equivalent circuit for utility and customer networks [13]. 
Harmonic voltage and current phasor measurements are taken at the PCC over a period of time. The harmonic current phasor $\boldsymbol{I}_{P h}$ is split into the harmonic current originating from the utility and from the customer (load), using harmonic impedances of the utility network and the customer's network as illustrated in Figure 2-10.

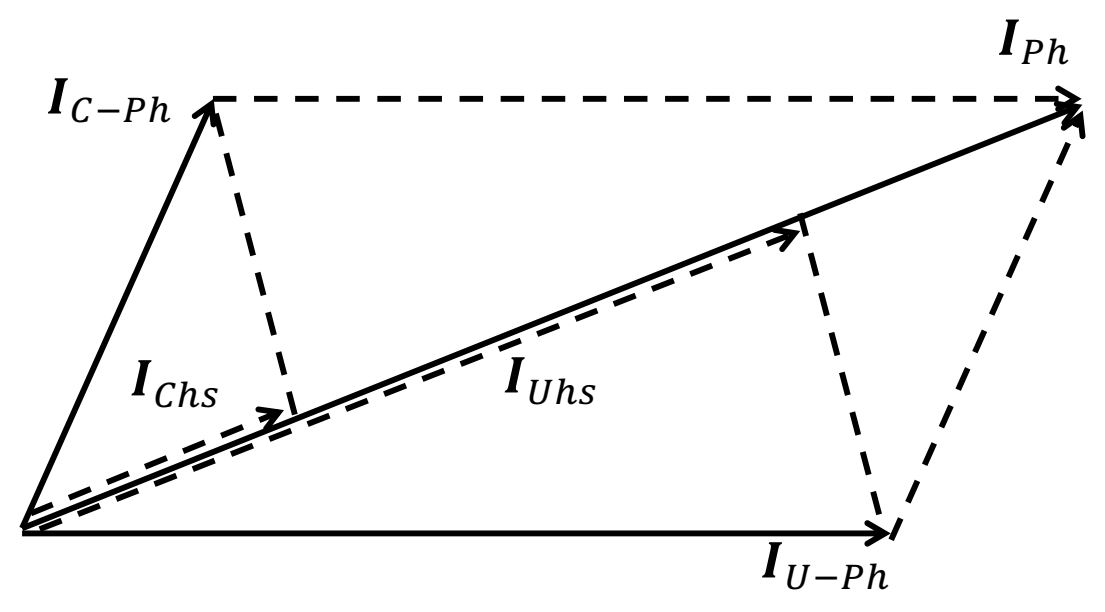

Figure 2-11. Example of utility and customer contributions to the measured current phasor at the PCC [13]

The respective contributions by the utility network or by the customer network are calculated using (8) and (9) below.

$$
\begin{gathered}
\boldsymbol{I}_{U h}=\frac{\boldsymbol{U}_{P h}}{\boldsymbol{Z}_{U h}}+\boldsymbol{I}_{P h} \\
\boldsymbol{I}_{C h}=\frac{\boldsymbol{U}_{P h}}{\boldsymbol{Z}_{C h}}-\boldsymbol{I}_{P h}
\end{gathered}
$$

Where;

$\boldsymbol{I}_{U h}$ : harmonic current phasor from utility network

$\boldsymbol{I}_{C h}$ : harmonic current phasor from load

$\boldsymbol{Z}_{U h}$ : complex harmonic impedance of utility network

$\boldsymbol{Z}_{C h}$ : complex harmonic impedance of load

$\boldsymbol{U}_{P h}$ : measured harmonic voltage phasor at PCC

$\boldsymbol{I}_{P h}$ : measured harmonic current phasor at the PCC

It is known that the harmonic impedance of the utilities network is not fixed due to changes in network configuration. Explanations are given in [70] for when network impedances are static and when they vary. Harmonic emission assessment under these varying harmonic impedances may lead to inconclusive results, as harmonic distortion levels may change depending on the network or loads contingency.

Reactive compensation can continuously change for example when controlling voltage or PF, and that will also change the harmonic impedance of the network. Fixed source impedances are assumed when passive harmonic filters are designed and when harmonic emission limits are apportioned to distorting loads.

In the case of varying network impedance, a reference impedance is proposed [70]. The principle of selecting a reference impedance is such that the customer or load must not be negatively 
impacted due to the operation of network capacitor banks or any other network contingencies. Customers or loads should not be held accountable for the change in the harmonic load flow due to changes in the upstream network, and for this reason a fixed refence impedance is selected. Selection of reference impedances must also be of such a nature as to not give the customer or load any benefit which will reduce the harmonic current emanating from the load. It is implied in [70] that the reference impedance should be selected as the impedance of the utilities network under normal operating conditions.

For an effective application of the HVM method, the network and load impedances must be known. These impedances are not always available with sufficient certainty [13]. Measurements needed for harmonic emission assessment normally require a considerable period of time during which the exact network conditions and changes must be well known in order to evaluate the harmonic emission correctly, the same for the load side impedances.

Capacitor bank switching or network outages (causing resonances and changes in harmonic impedances) may affect the harmonic measurements leading to incorrect results if information on impedances and the changes thereof were not accurate.

Of importance to note is that this method does not deal with the harmonic emission of a non-linear load, but rather how the measurement harmonic distortion is constituted. The next section deals with the calculation of the harmonic emission from the non-linear load through superposition.

\subsubsection{Superposition method}

The superposition method is an extension of the HVM method described under section 2.5.4 The measured harmonic current $\boldsymbol{I}_{P h}$ is split into a component from the customer side $\left(\boldsymbol{I}_{C-P h}\right)$ and from the utility side $\left(\boldsymbol{I}_{U-P h}\right)$ indicated in Figure 2-12.

Application of the superposition principle is used to determine what the contribution of each harmonic source is, i.e. the harmonic emission.
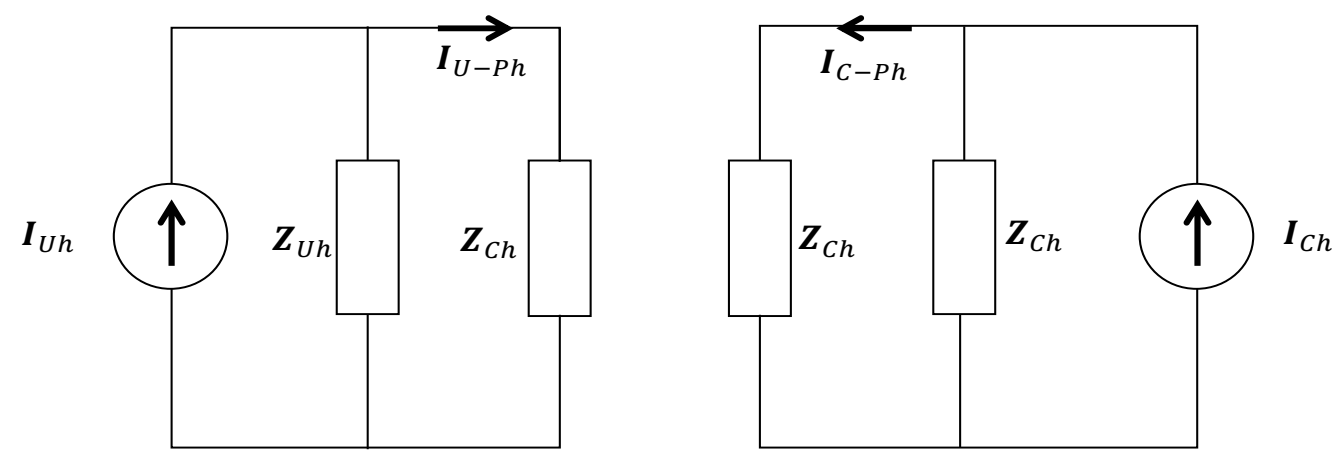

Figure 2-12. Customer and utility harmonic current contribution using superposition principles [13]

$$
\begin{gathered}
\boldsymbol{I}_{U-P h}=\frac{\boldsymbol{Z}_{U h}}{\boldsymbol{Z}_{U h}+\boldsymbol{Z}_{C h}} \boldsymbol{I}_{U h} \\
\boldsymbol{I}_{C-P h}=\frac{\boldsymbol{Z}_{C h}}{\boldsymbol{Z}_{U h}+\boldsymbol{Z}_{C h}} \boldsymbol{I}_{C h} \\
\boldsymbol{I}_{P h}=\boldsymbol{I}_{U-P h}+\left(-\boldsymbol{I}_{C-p h}\right)
\end{gathered}
$$

Where:

$\boldsymbol{I}_{U-P h}:$ is the harmonic current phasor contribution from the utility network to the PCC 
$\boldsymbol{I}_{C-P h}: \quad$ is the harmonic current phasor contribution from the customer to the PCC

A combination of the HVM and superposition is required to evaluate the harmonic emission of a non-linear load as applied in Chapter 3.

\subsubsection{RLC}

The RLC method is used to determine the load's (at customer/user) harmonic impedance [14]. It makes use of harmonic voltages and current phasors measured at the PCC to calculate the resistive, capacitive and inductive components that represent the load impedance as shown in Figure 2-13. The method is aimed at improving the harmonic vector method by quantifying the harmonic impedance of the load through measurements. This is useful, as the load or customer impedance is often difficult to determine [13]. Note that this is not a harmonic emission assessment method, as the method only focuses on the harmonic impedance of the load.

The harmonic impedance of the supply network can be obtained from the utility, from computer simulations or by measurements at the PCC detecting harmonic voltage changes that are a consequence of harmonic current changes [13].

With the load and network impedances obtained, (13) to (15) are used to calculate the load or customer side contributions towards the total harmonic current measured at the PCC. Firstly, the resistive component is calculated based on active power equations. The remaining capacitive and inductive components are calculated based on reactive power equations.

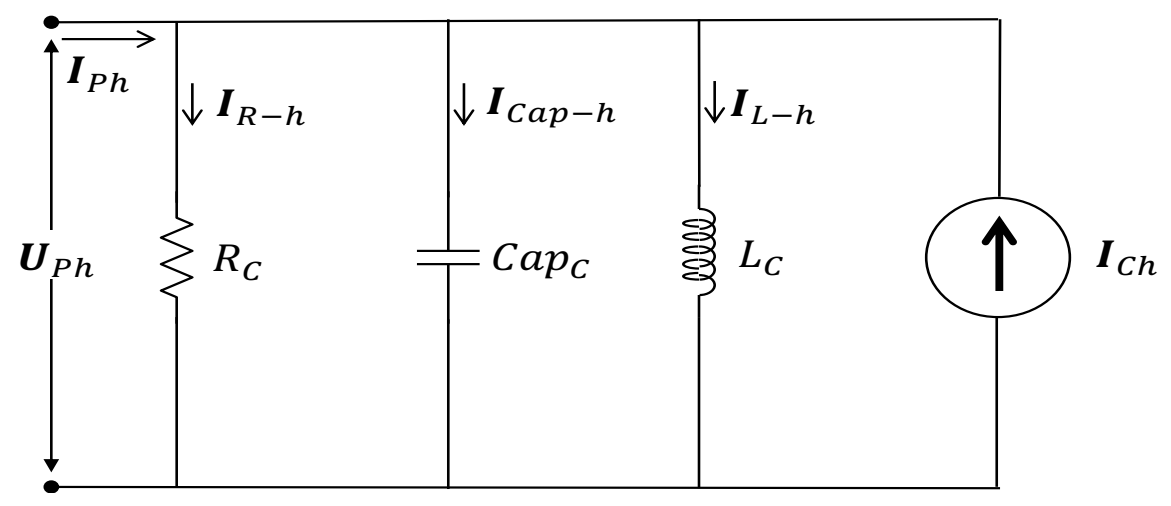

Figure 2-13. RLC customer equivalent circuit [14]

$$
\begin{gathered}
R_{c}=\frac{\sum_{h=1}^{H}\left(U_{P h}\right)^{2}}{\sum_{h=1}^{H} U_{P h} I_{P h} \cos \left(\varphi_{h}\right)}, \quad R_{c}>0 \\
\operatorname{Cap}_{c}=\frac{\sum_{h=1}^{H} h U_{P h} I_{C L I h} \sin \left(-\varphi_{h}\right)}{\omega \sum_{h=1}^{H}\left(U_{P h}\right)^{2} h^{2}}, \quad \operatorname{Cap}_{c}>0 \\
L_{c}=\frac{\sum_{h=1}^{H}\left(U_{P h}\right)^{2} / h^{2}}{\omega \sum_{h=1}^{H} h U_{P h} I_{L I h} \sin \left(\varphi_{h}\right) / h}, \quad L_{c}>0
\end{gathered}
$$

$\varphi_{h}$ is the difference in the harmonic voltage phase angle and harmonic current phase angle at harmonic order $h$.

Where:

$$
I_{C L I h}=I_{P h}-\frac{U_{P h}}{R_{C}}
$$




$$
\begin{gathered}
I_{L I h}=I_{C L I h}-\frac{U_{P h}}{Z_{c a p}} \\
I_{C h}=I_{L I h}-\frac{U_{P h}}{Z_{L}}
\end{gathered}
$$

The method assumes that all the resistive, capacitive and inductive components are in parallel, but depending on where the measurements are taken, the components may be arranged in a series configuration rendering this method useless. This is the case for passive harmonic filters.

\subsubsection{CIGRE/CIRED C4.109 scatterplot (statistical) method}

The CIGRE/CIRED C4.109 method is based on the HVM method discussed in section 2.5.4. The utility network is now modelled as a Thévenin equivalent network rather than a Norton equivalent as shown in Figure 2-14.

The utility harmonic impedance is used to calculate the voltage harmonic emission emanating from the nonlinear load as indicated in (19). The harmonic current used in the voltage emission calculation is measured at the PoC, as depicted in Figure 2-14.

$$
\left|\boldsymbol{E}_{h c}\right|=Z_{h} I_{h}=\left|\boldsymbol{V}_{\boldsymbol{h}}-\boldsymbol{E}_{\boldsymbol{h 0}}\right|
$$

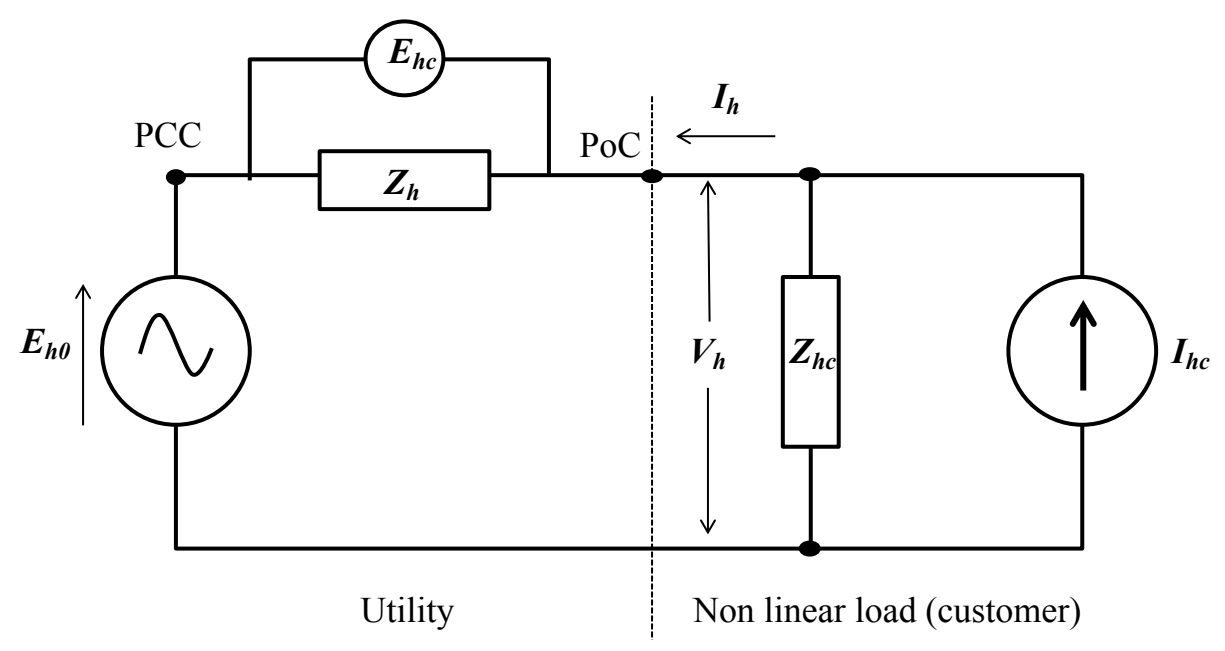

Figure 2-14. Equivalent network diagram for the definition of the harmonic emission level at the PoC [19]

Where:

$V_{\boldsymbol{h}}$ : harmonic voltage phasor at the PoC

$\boldsymbol{I}_{\boldsymbol{h}}: \quad$ harmonic current phasor

$\boldsymbol{E}_{h 0}$ : background (in the supply system) harmonic voltage phasor at Point of Common Coupling (PCC)

$\boldsymbol{Z}_{h}: \quad$ complex supply impedance

$\boldsymbol{Z}_{h c}$ : complex impedance (consumer/RPP installation)

$\boldsymbol{I}_{h c}$ : harmonic current phasor from consumer/RPP installation

$\boldsymbol{E}_{h c}$ : voltage harmonic emission phasor 
Harmonic current is considered as emission when $\left|\boldsymbol{V}_{h}\right|>\left|\boldsymbol{E}_{h 0}\right|$, the harmonic current is flowing from the nonlinear load into the upstream network. This condition is not easily confirmed with a single-point measurement at the PoC, as $\boldsymbol{E}_{h 0}$ is unknown. If multiple measurements are done at the PoC and the PCC simultaneously (synchronously), then the condition may be applied.

Voltage harmonic emission assessment as described above, requires the measurement of phasors. However, in recognizing that power quality recorders that employ the IEC 61000-4-30 [49], the 10-minute aggregation method is limited to only rms harmonic voltage and current measurement. Therefore, the CIGRE/CIRED C4.109 also proposed a statistical approach to evaluate the major contribution towards the harmonic emission at a PCC.

The C4.109 statistical method requires knowledge of the load and network impedance as well as measurement at the PCC for the evaluation. Harmonic voltage and current rms measurements at 10-minute intervals are plotted on a scatterplot with the harmonic current measurements on the $\mathrm{x}$ axis and the corresponding harmonic voltage measurements on the y-axis, done per harmonic order as shown in Figure 2-15. Harmonic impedance of the utility network and that of the load or customer network at the harmonic order being evaluated, are plotted onto the same scatterplot.

Impedance slopes are used to qualitatively assess who the major contributor towards the harmonic distortion at the PCC is. If all the scatter points are concentrated around the slope of the network impedance $\left|\boldsymbol{Z}_{h}\right|$, then the load is considered to be the major contributor, but if the scatter points are concentrated around the load or customer impedance $\left|\boldsymbol{Z}_{h c}\right|$, then the utility network is the major contributor towards harmonic distortion. If the scatterplots concentrate between the impedance slopes, then both the load customer and the utility network contribute towards harmonic distortion.

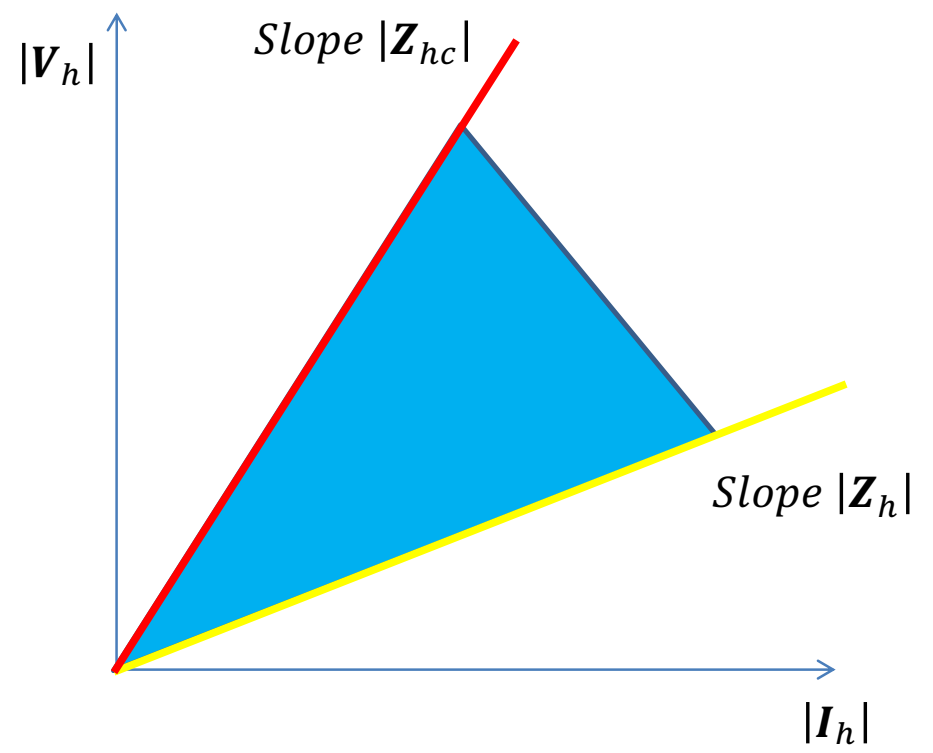

Figure 2-15. Principle of the CIGRE/CIRED C4.109 method [19]

This method is only qualitative and exact quantification of how much different sources of harmonics (utility/load) contributes is not possible. If impedance slopes are not located on top of the scattered points, no specific contribution can be blamed. Application of this method with the goal to assess contractual emission values, is not possible. It can however aid to better understand where the dominant source of a specific harmonic is (upstream/downstream).

As discussed above, accurate harmonic impedance data are not always available [13]. It is reported in [71] that only the network impedance is required for a conclusive result. The CIGRE/CIRED C4.109 document [72] states that a transformer impedance between the utility PoC and the load may be used as the load impedance in the absence of detailed impedance information. 


\subsubsection{Range of secondary cancellation: when does harmonic matter?}

In [73] the novel concept of secondary cancellation is detailed in theory, where the harmonic current phasor results in a reduction in the subsequent harmonic voltage at a PoC or PCC. The aim of this concept or technique is to determine the range of harmonic current phase angles which will result in a reduction of the harmonic voltage at the PoC being assessed. Discrimination of harmonic emission values can be achieved with this method as only harmonic emission phasor values (from a nonlinear load) which have a positive impact on the source network should be reported.

The Range of Secondary Cancellation (RSC) concept is based on the simplified network diagram depicted in Figure 2-16, which is similar to Figure 2-14. It is assumed that the current at the power frequency is flowing into the load.

Focus on grid code compliance should only cater for harmonic distortion that has a negative impact on the network.

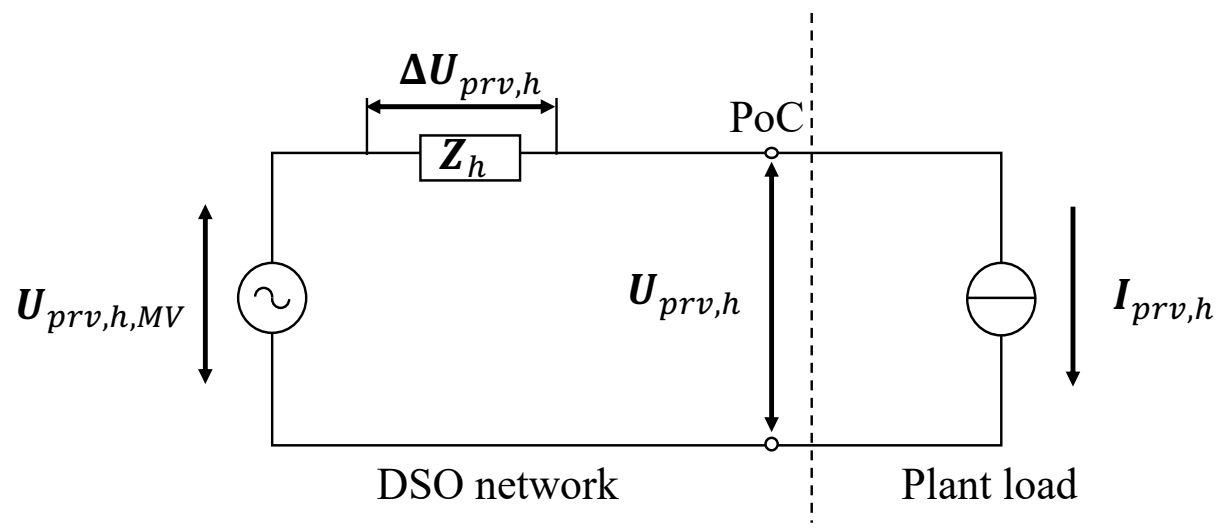

Figure 2-16. Simplified single-line equivalent circuit (adapted from [73])

Theory discussed in [73] calculates the harmonic voltage $\boldsymbol{U}_{p r v, h}$ at the load by using the zero sequence harmonic impedance of the network. If the zero sequence impedance is constant [73], then the harmonic voltage is determined by the harmonic current $\boldsymbol{I}_{p r v, h},(20)$ applies.

$$
\boldsymbol{U}_{\text {prv }, h}=-\boldsymbol{Z}_{0, h} \boldsymbol{I}_{\text {prv }, h}
$$

In a complex network with multiple nonlinear loads, cancellation of harmonics exists, as not all of the harmonic phase angles of the different loads are the same [16]. Should there be a high degree of diversity of the harmonic current phasors, then the resultant harmonic current used in (20) will result in a lower harmonic voltage, due to the dynamic interaction of harmonic current phasors between loads.

Similar arguments can be made with the positive and negative sequence harmonic impedances to determine the background harmonic voltage as in (21). The background harmonic voltage must be taken into consideration when secondary cancellation is calculated.

$$
\boldsymbol{U}_{p r v, h}=\boldsymbol{U}_{p r v, h, M V}-\boldsymbol{Z}_{2, h} \boldsymbol{I}_{p r v, h}
$$

The minimisation of the harmonic voltage due to the harmonic current and the background harmonic voltages are given in (22);

$$
\min \left[U_{p r v, h, M V} \cdot e^{j \varphi_{U p r v, h, M V}}-I_{p r v, h} \cdot Z_{2, h} \cdot e^{j\left(\varphi_{I p r v}+\psi_{Z 2, h}\right)}\right]
$$


Secondary cancellation is illustrated in Figure 2-17 and Figure 2-18.

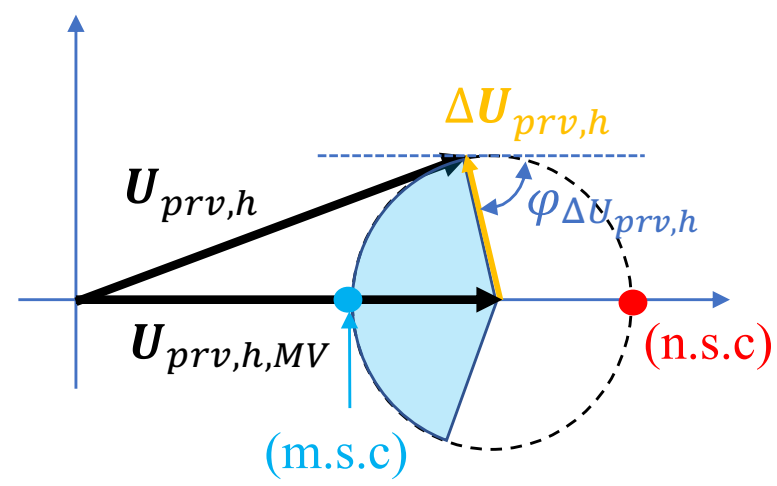

Figure 2-17. Harmonic voltage phasor diagram of (21) [73]

n.s.c - no secondary cancellation

m.s.c - maximum secondary cancellation

Maximum secondary cancellation (m.s.c) of the harmonic voltage is achieved when the phase angle of the voltage drop $\Delta \boldsymbol{U}_{p r v, h}$ is $180^{\circ}$ out of phase with the background phase voltage $\boldsymbol{U}_{\text {prv }, h, M V}$, as indicated by the blue dot on Figure 2-17. No secondary cancellation occurs when the phase angle $\Delta \boldsymbol{U}_{p r v, h}$ is in phase with $\boldsymbol{U}_{p r v, h, M V}$. If $\Delta \boldsymbol{U}_{p r v, h}$ operates in the highlighted blue area, then secondary cancellation of $\boldsymbol{U}_{p r v, h, M V}$ takes place.

Measurement of harmonic voltage drop $\Delta \boldsymbol{U}_{p r v, h}$ in a practical power system cannot be done, as the definition and location of $\boldsymbol{U}_{p r v, h, M V}$ and $\boldsymbol{Z}_{h}$ are theoretical parameters. Harmonic current $\boldsymbol{I}_{p r v, h}$ and $\boldsymbol{U}_{p r v, h}$ are the only two quantities to be measured relatively accurately if measurement uncertainty in the instrumentation chain is well contained. The RSC for harmonic current is calculated by (23).

$$
\varphi_{I, p r v, h(\max , \min )}=\left(\varphi_{U, p r v, h, M V}-\psi_{Z 2, h}\right) \pm \cos ^{-1}\left(\frac{I_{p r v, h} * Z_{2, h}}{2 * U_{p r v, h, M V}}\right)
$$

Where:

$\varphi_{U, p r v, h, M V}$ : background voltage harmonic phase angle of supply network

$\psi_{Z 2, h}$ : angle of the network impedance at the assessed harmonic, obtained by simulation of the network components.

$I_{p r v, h}$ prevailing harmonic current magnitude

$Z_{2, h}$ : network impedance magnitude at the assessed harmonic, obtained by simulation of the network components

$U_{\text {prv }, h, M V}$ : background voltage magnitude of the network

Applying (23) to a nonlinear load will result in a minimum and maximum phase angle for the current harmonics in which secondary cancellation will occur, as highlighted by the blue area in Figure 2-18. 
Inclusion of the harmonic impedance at the $\mathrm{PoC}$ and the background voltage harmonic distortion is a more accurate approach that focuses the RSC calculation to a specific PoC. Accuracy of this method is, however, dependent on accurate network harmonic impedance information at the PoC.

RSC theory is based on a "normal" load flow application, but with renewable power, the flow is in the opposite direction. The sign of the prevailing harmonic currents must be changed by 180 degrees for distributed energy source applications.

\subsection{The measurement of harmonics}

It is not only the harmonic emission assessment methodology that is important to understand, but also the metrological aspects behind the harmonic emission assessment. In this section the various measurement approaches are discussed as well as the impact of instrument transformers on the measurements.

\subsubsection{IEC 61000-4-30 and IEC 61000-4-7: How harmonics are measured by engineers}

The international standards body, the IEC, published measurement standards for the measurement of harmonics and stipulates the implementation of those measurements in a measuring instrument (normally referred to as a PQ instrument). By this harmonisation, field data obtained from different instruments are comparable when the instrument and the measurement methodology are compliant with the measurement requirements of the IEC 61000-4-30 [49] and IEC 61000-4-7 [74] standards.

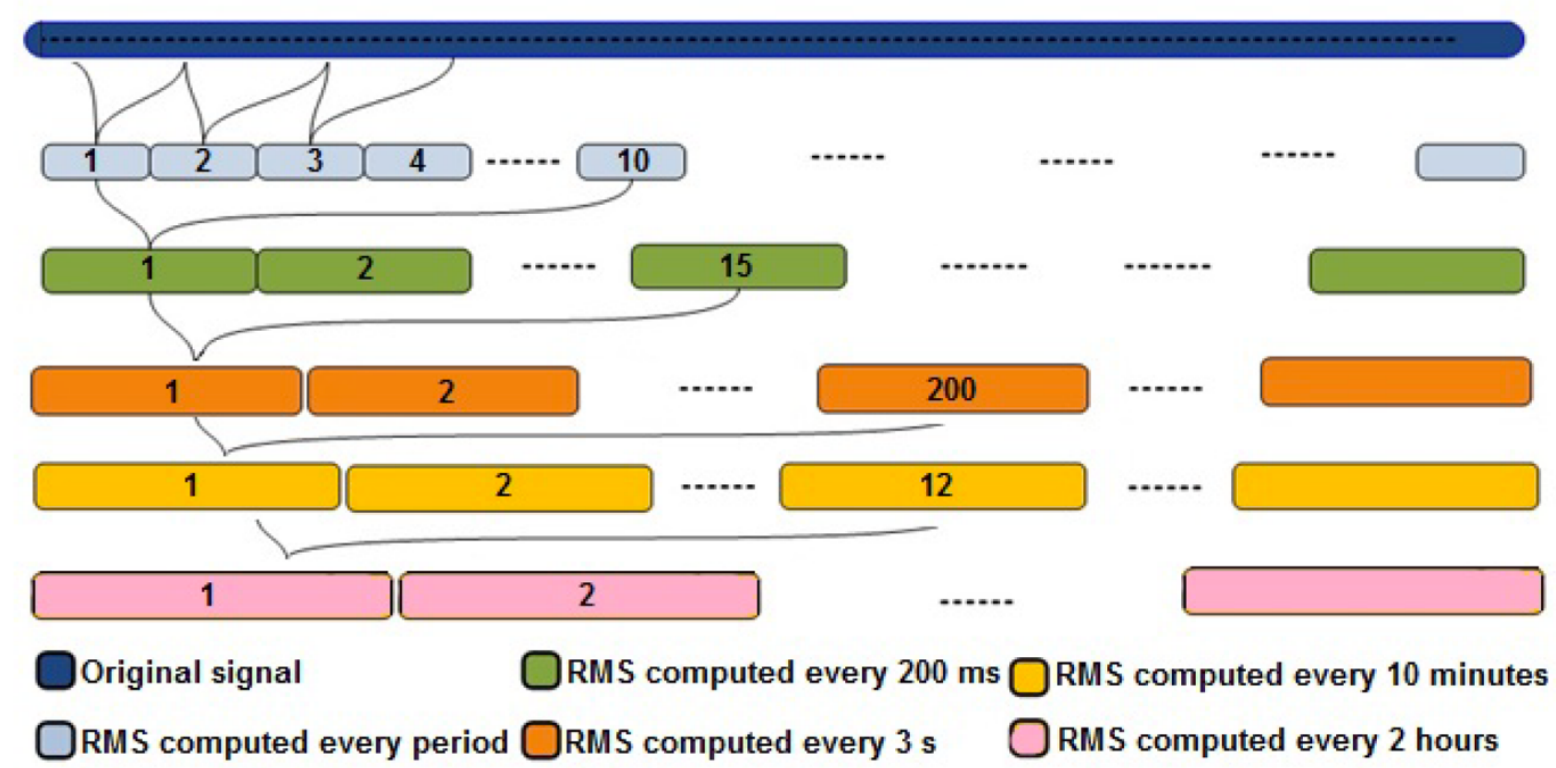

Figure 2-19. IEC 61000-4-30 aggregation principles [75].

Harmonic information is obtained by conducting a Fourier analysis on the $200 \mathrm{~ms}$ (often called the 10/12-cycle data point) data sample. During this Fourier analysis process, the phase angle information is discarded and only the rms information is retained by the PQ instrument. Depending on the PQ instrument used, the rms data may be reported in several measurement intervals, either in 3-sec, 1-min, 10-min or 2-hour aggregation points. Users can select which data interval is required, depending on the application.

In most cases electrical utilities will default to the 10-min rms data points as suggested by the IEC 61000-4-30 standard. For the purpose of this analysis, the 10-min rms data interval will be used for analysis. 


\subsubsection{Single-point measurements}

Harmonic measurements using a single PQ instrument placed in a single node within a network is referred to as a single-point measurement. Table 2-2 lists the type of measurement campaigns to be employed for the different harmonic emission assessment methods, with some using multiplepoint and single-point measurements.

Single-point measurements are easier to implement and the most cost-effective method, due to the reduced number of PQ instruments required. Note that the methods for single-point measurements listed in Table 2-2, all require the measurement of harmonic phasors.

A PQ instrument is commonly used for monitoring harmonics in power systems and even if certified to be compliant with the IEC 61000-4-30 PQ measurement standard [49], it can happen that it only retains aggregated PQ parameters without phase angle information. Using rms values for harmonic phasors may not give useful results on harmonic emission, as pointed out later on in Chapter 4 of this thesis.

Previous versions of the IEC 61000-4-30 measurement standard did not require the measurement of current for an instrument to be Class A compliant. It was only during edition 3, published in 2015 [49], that the Class A measurement requirements for current were specified. Earlier instruments could measure current, but how well the metrology performed was not a certification requirement, and as such, the measurement uncertainty was invalidated.

Electrical utilities interested in monitoring their network's PQ performance will typically deploy a single PQ instrument at a PCC where various load types are connected. Such a measurement campaign is not to determine which party is causing the PQ pollution, but rather to monitor the performance levels of the network.

It was shown in [16] that when using harmonic active power to evaluate harmonic emission in a network with nonlinear loads distributed all over, synchronous measurements at all points of interest in that network are required because of the exchange of harmonic active power between different nonlinear loads.

Single-point measurements remain the most accessible to utilities and this is the reason why this thesis aims to develop an innovative method that can be used to improve the existing deficiencies of harmonic emission assessment methods by using aggregated harmonic phasors, as presented later on in this thesis.

\subsubsection{Multiple-point measurements}

A PQ measurement campaign using multiple-point measurements is cumbersome and time consuming due to the use of multiple PQ instruments located at different locations in an electrical network. This approach is, however, a more accurate means to determine which party is causing the harmonic pollution at a PCC, even if the harmonic source is located elsewhere in the network [76], [77]. Harmonic contributions from individual feeders or circuits can be evaluated when both harmonic voltage and harmonic currents (magnitude and phase angle) are measured.

In [78], synchronous multiple-point measurements are being employed in conjunction with superposition theory to determine the source of harmonic pollution within a steel plant. The voltage and current harmonics phasors are measured on each feeder synchronously and a Norton equivalent for each feeder is determined, as depicted in Figure 2-20. 


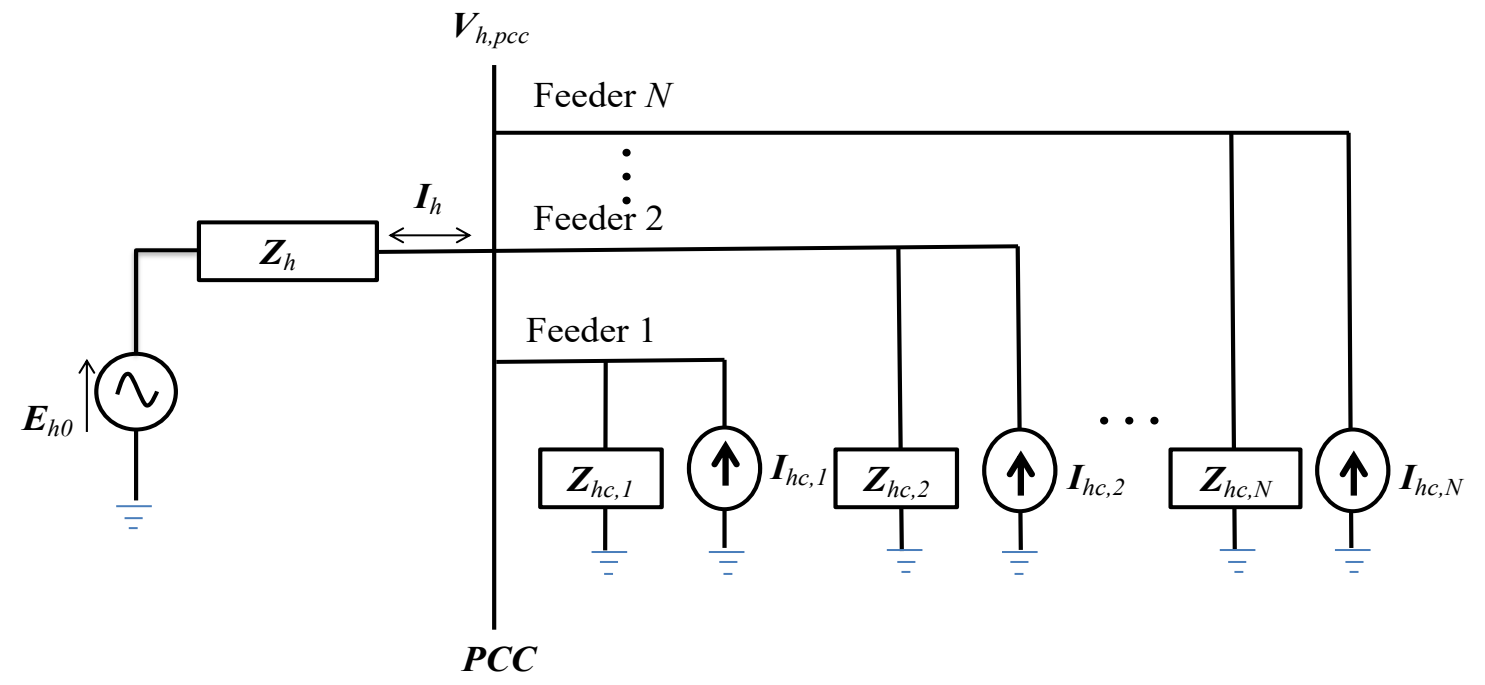

Figure 2-20. Multiple-point measurement system (adapted from [78])

When using multiple-point measurements, care must be taken to ensure that each measurement is taken at the same time. The application of GPS systems [79] ensures synchronization between the various PQ measurements, and it ensures that each time stamped measurement data point is taken under the same network conditions for ease of comparison and analysis.

Implementation of the CIGRE C4.109 scatterplot method requires that $\left|\boldsymbol{V}_{h}\right|>\left|\boldsymbol{E}_{h 0}\right|$, however, with single-point measurements this condition cannot be confirmed, as only $V_{h}$ is measured (rms only) and $E_{h 0}$ is assumed. A multiple-point measurement campaign must be undertaken to ensure that the conditions of the $\mathrm{C} 4.109$ method are fulfilled, even though only 10-minute aggregated values are used.

\subsubsection{Measurement of harmonic phase angles}

IEC 61000-3-12 [39] describes the measurement of harmonic phase angles with reference to the phase to neutral fundamental voltage. The phase angle is measured from the zero crossing of the fundamental signal to the zero crossing of the harmonic signal, as illustrated in Figure 2-21 in which the signal shown is at the time when the harmonic current leads the fundamental voltage. The resulting phase angle measurement will be a positive value $\left(\alpha_{5}>0\right)$. Figure 2-22 shows the condition when the harmonic current lags the fundamental voltage, the phase angle in this case will be a negative value $\left(\alpha_{5}<0\right)$. 


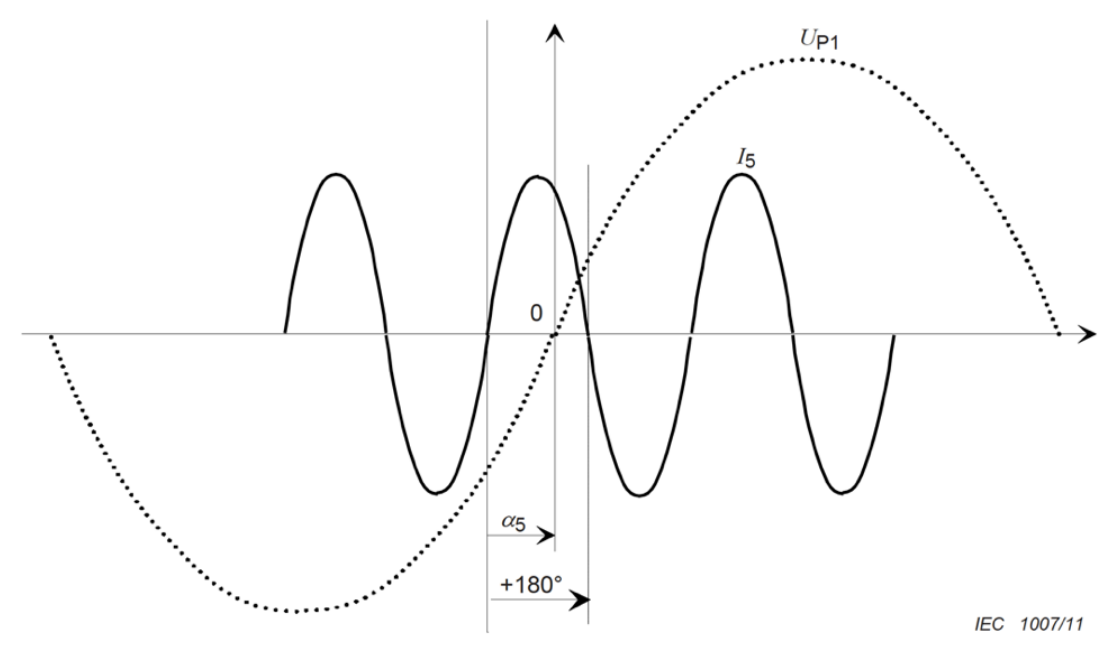

Figure 2-21. Definition of the $5^{\text {th }}$ harmonic current phase angle for Is leading $U_{P I}$

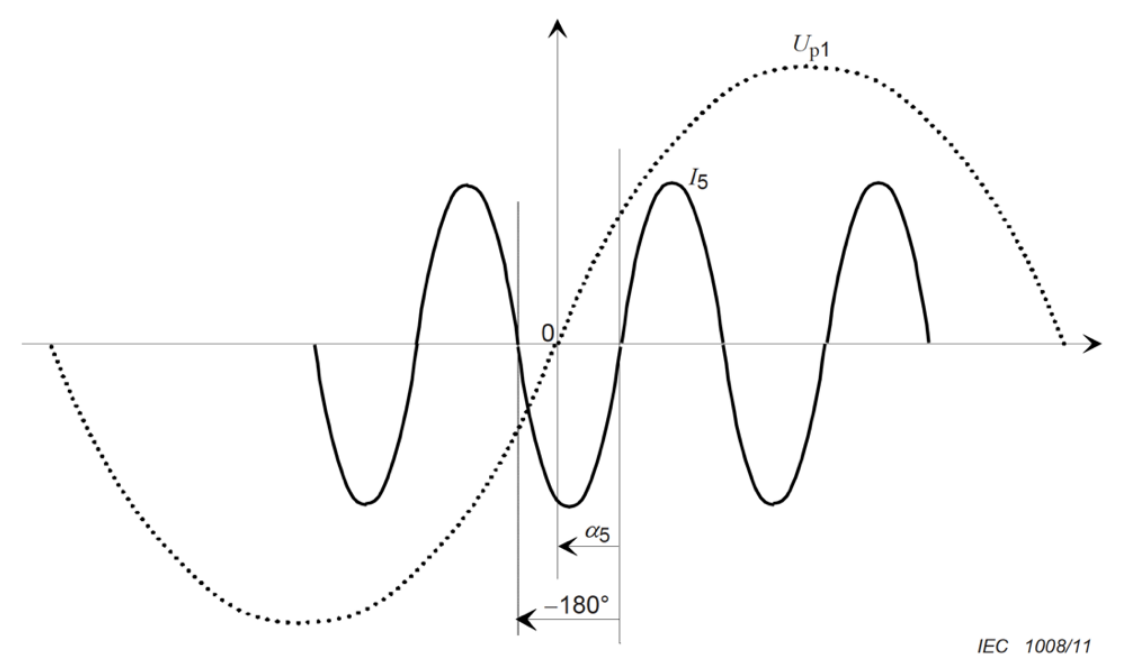

Figure 2-22. Definition of the $5^{\text {th }}$ harmonic current phase angle for $I_{5}$ lagging $U_{P I}$

IEC 61000-4-7 [74] defines the spectral component magnitude in the frequency domain. In [80] the measurement of harmonic rms values are further discussed, emphasising the application of a Fourier transform to calculate the harmonic rms values for every 10 cycles in a $50 \mathrm{~Hz}$ system (12 cycles for a $60 \mathrm{~Hz}$ system), resulting in the harmonic distortion values as recorded by modern day PQ recorders.

\subsection{Measurement uncertainty}

It is not sufficient to report the measurement value alone, the reliability or completeness of the measured values must also be quantified for a comparative analysis to be concluded [81]. Uncertainty in measurement or doubt [82] is a value that is associated with the measurand. The measurand is the quantity to be measured, in the context of this document, the measurands are the measured harmonic voltage and harmonic current values.

Standard uncertainty is defined as Uncertainty of the result of a measurement expressed as a result of a standard deviation [81], [82]. Typically, a Probability Density Function (PDF) is applied to the measured data to represent the distribution of the data. The standard deviation is applied to the mean of the measured data as a simple approach to the quantification of measurement uncertainty. Equation (24) illustrates this concept, where $\bar{x}$ is the mean value and $u(x)$ is the standard deviation. 


$$
\bar{x}-u(x), \quad \bar{x}+u(x)
$$

The uncertainty assessment is further impacted by the shape of the PDF, (24) holds true for a normal distribution. For a rectangular distribution and a triangular distribution, equations (25) and (26) respectively hold true for the deviation [81].

$$
\bar{x}-\frac{a}{\sqrt{3}}, \quad \bar{x}+\frac{a}{\sqrt{3}}
$$

Where $a$ is half the width of the rectangle.

$$
\bar{x}-\frac{a}{\sqrt{6}}, \quad \bar{x}+\frac{a}{\sqrt{6}}
$$

Where $a$ is half the width of the triangle.

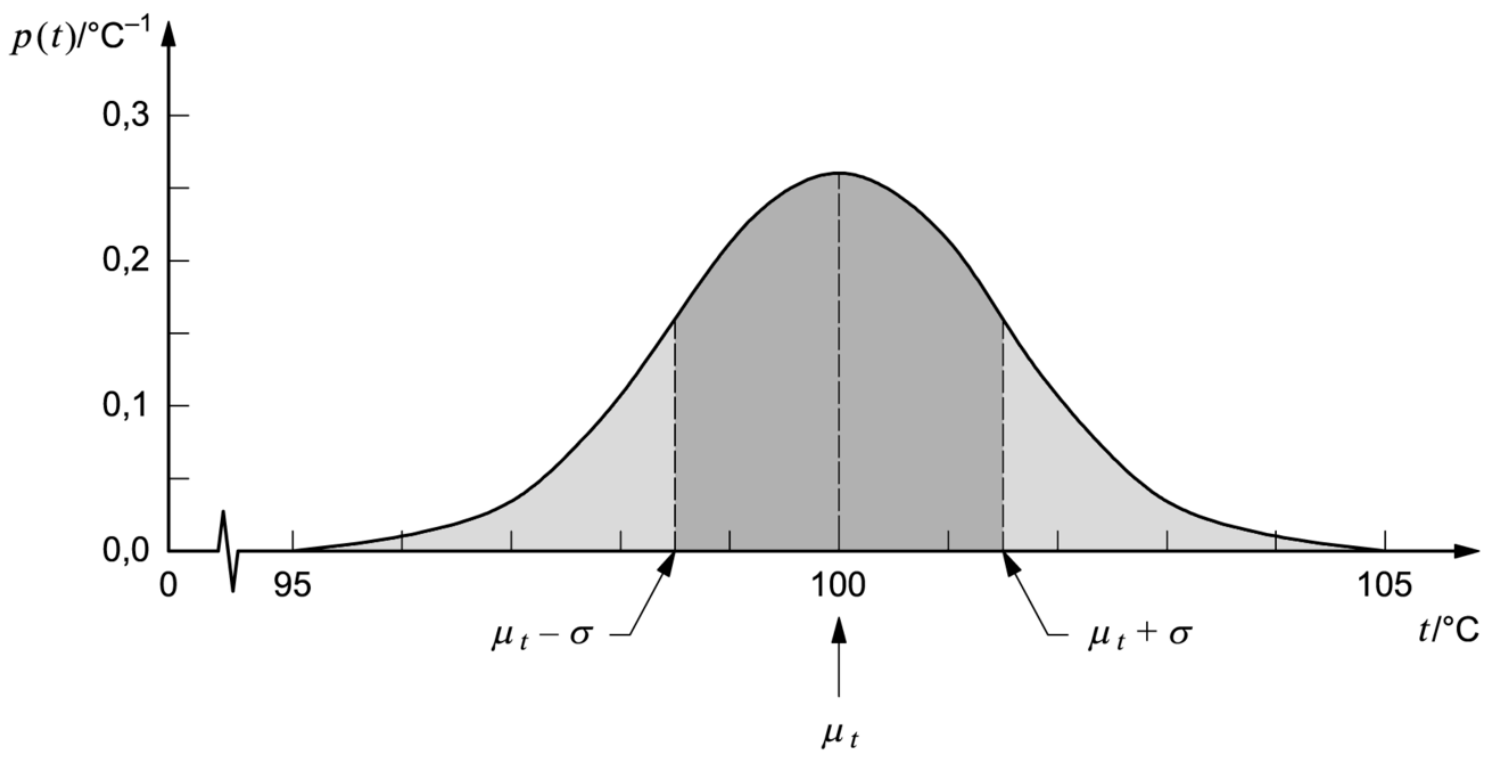

Figure 2-23. Normal distribution [81] 


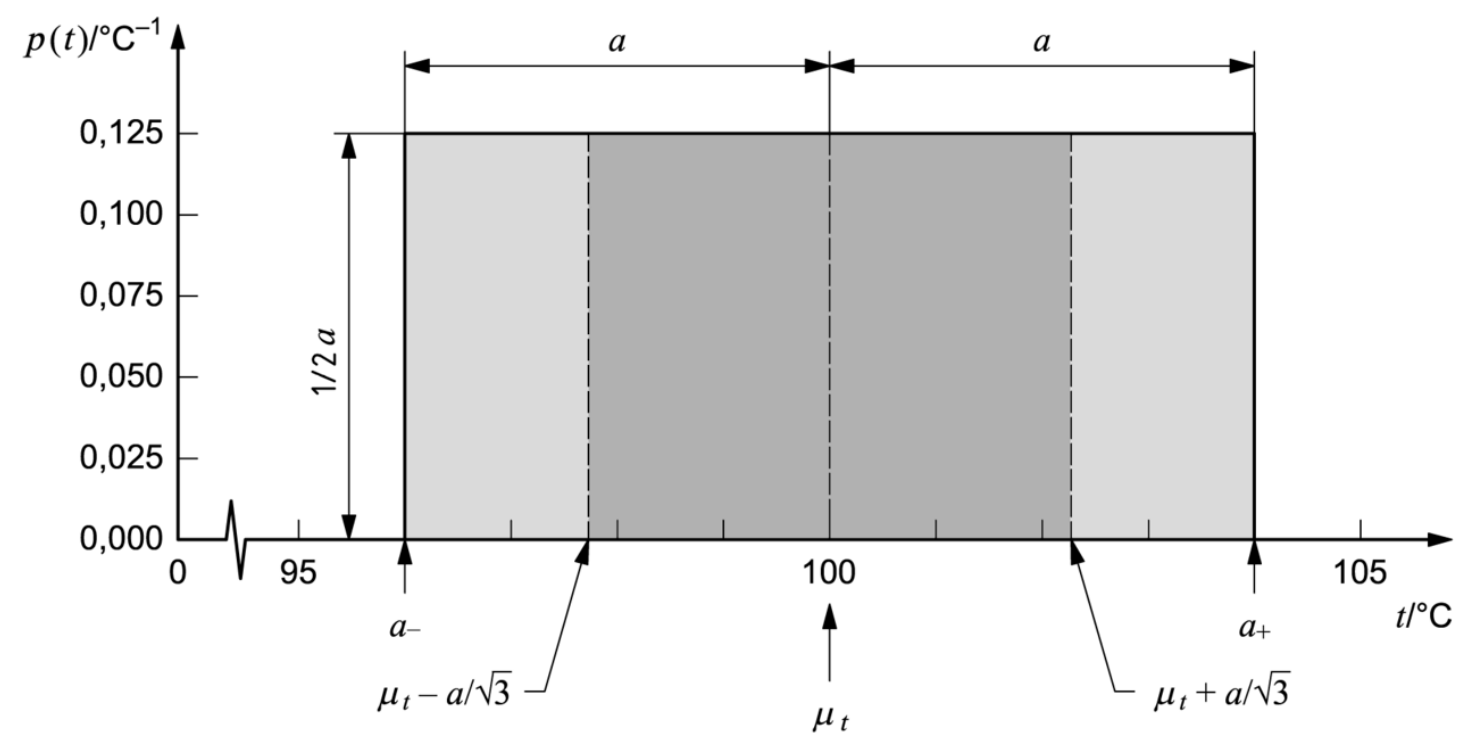

Figure 2-24. Rectangular distribution [81]

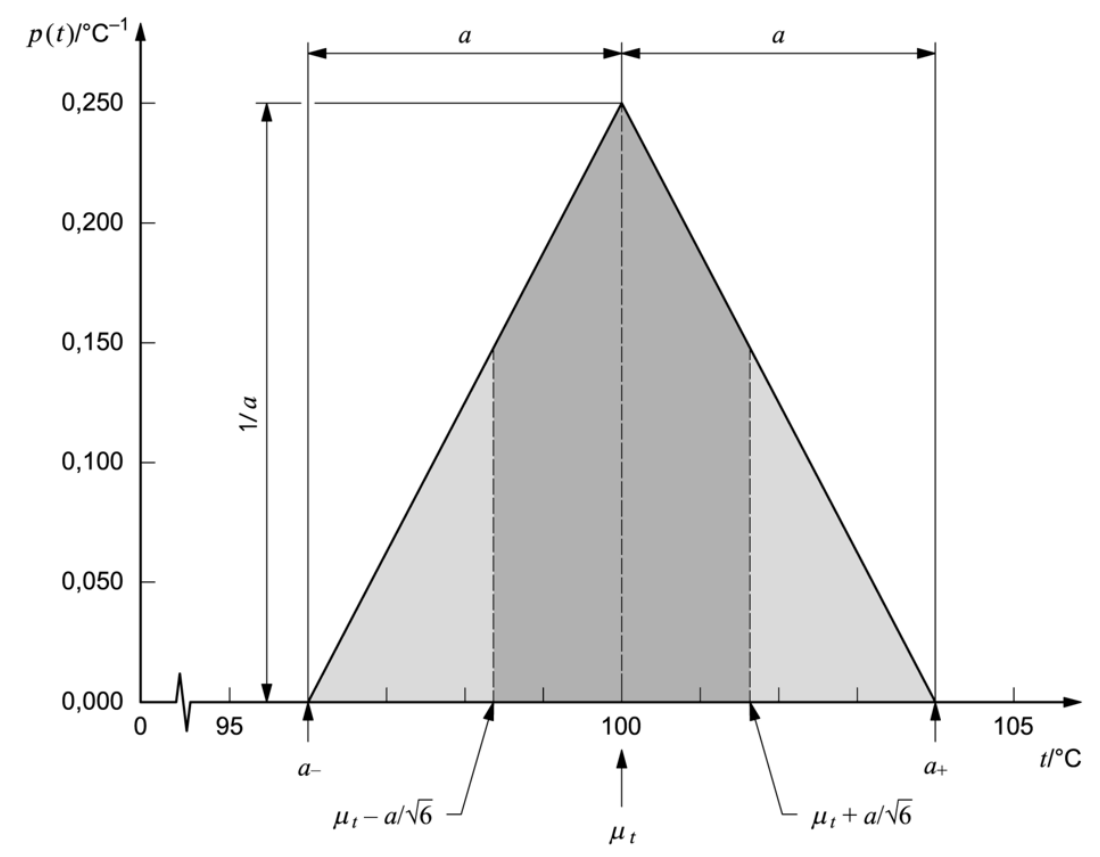

Figure 2-25. Triangular distribution [81]

Expanded uncertainty is defined as a quantity defining an interval concerning the result of a measurement that may be expected to encompass a large fraction of the distribution of values that could reasonably be attributed to the measurand [81], [82]. The fraction applied can be viewed as a coverage probability or level of confidence of the defined interval.

Expanded uncertainty is calculated by multiplying the standard uncertainty with a suitable coverage factor $\mathrm{K}$. The coverage factor is defined as a numerical factor that is used as a multiplier of the standard uncertainty in order to obtain an expanded uncertainty [82], [81]. The coverage factor is dependent on the shape (normal distribution or rectangular) of the PDF for the 
measurements. Typical coverage factors when a normal distribution is assumed are listed in Table 2-3. Table 2-4 list the coverage factors when a rectangular distribution is observed.

Table 2-3. Coverage factor when assuming a normal distribution [82]

\begin{tabular}{|c|c|}
\hline Level of confidence p (\%) & Coverage factor $\boldsymbol{K}_{\boldsymbol{p}}$ \\
\hline 68.27 & 1 \\
\hline 90 & 1.645 \\
\hline 95 & 1.96 \\
\hline 95.45 & 2 \\
\hline 99 & 2.576 \\
\hline 99.73 & 3 \\
\hline
\end{tabular}

Table 2-4. Coverage factor when assuming a rectangular distribution [81]

\begin{tabular}{|c|c|}
\hline Level of confidence p (\%) & Coverage factor $\boldsymbol{K}_{\boldsymbol{p}}$ \\
\hline 57,74 & 1 \\
\hline 95 & 1.65 \\
\hline 99 & 1.71 \\
\hline 100 & 1.73 \\
\hline
\end{tabular}

The Guide to the Expression of Uncertainty in Measurement [81] describes various methods of determining the uncertainty in measurement. Combined Standard Uncertainty (CSU) [81], [82] (which is applicable in this case) is used when the measurand is not measured directly, as illustrated in Figure 2-29. CSU combines the errors of all of the equipment in the measurement circuit to determine an overall measurement uncertainty that represents the entire measurement circuit as calculated in (27).

$$
u_{c}^{2}(y)=\sum_{i=1}^{N}\left(\frac{\partial f}{\partial x_{i}}\right)^{2} u^{2}\left(x_{i}\right)
$$

Where:

$\left(\frac{\partial f}{\partial x_{i}}\right): \quad$ is the sensitivity coefficient

$u^{2}\left(x_{i}\right): \quad$ is the value of square of the equipment variance (often obtained from the specification sheet)

The sensitivity coefficient is used when a piece of equipment may have a larger influence on the measurement results. If the equipment has no effect larger than the other equipment, then the value is selected to be 1 . The influence of instrument transformers is reviewed next as it is an important consideration in the assessment of measurement uncertainty.

The frequency response of Voltage Transformers (VTs) is extensively reported and visualized in literature [83]-[86]. Results indicate that the measurement error [51] in magnitude and phase angle for VTs changes considerably above $1 \mathrm{kHz}$, but below $1 \mathrm{kHz}$ the frequency response remains fairly linear. Figure 2-26 and Figure 2-27 are extracts from [85] which illustrates the frequency response of VT at various voltage levels. If the error is known, off-line compensation can be done, but this requires calibration of the VT over the frequency range of interest. 


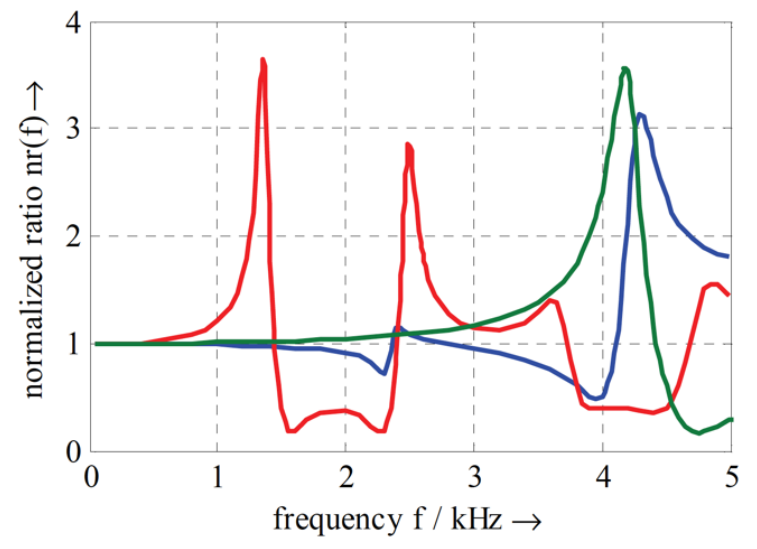

Figure 2-26. Extract from [85] showing normalised magnitude of the frequency response of voltage transformers at $66 \mathrm{kV}$ (green), $110 \mathrm{kV}$ (blue) and 220 $k V$ (red)

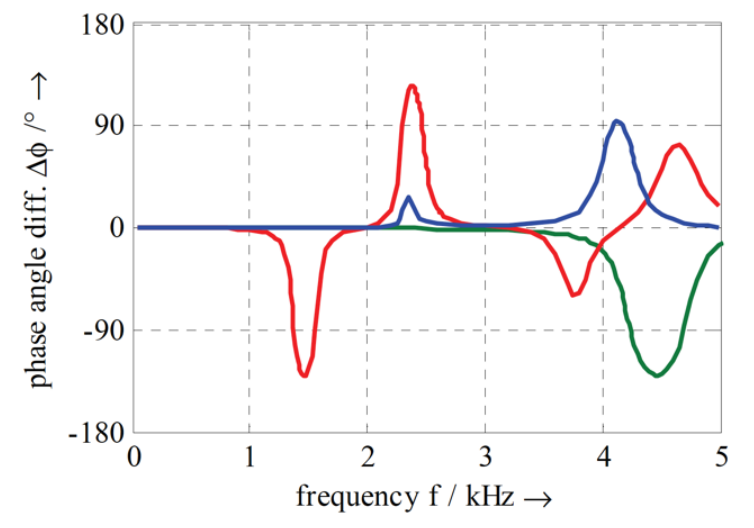

Figure 2-27. Extract from [85] showing phase angle difference of the frequency response of voltage transformers at $66 \mathrm{kV}$ (green), $110 \mathrm{kV}$ (blue) and 220 $k V$ (red)

Electrical utilities are mainly concerned with the low-order harmonic distortion below $2 \mathrm{kHz}$. VT errors are thus a real concern if the frequency response of the VT is not linear above $1 \mathrm{kHz}$.

It is reported in [84] that above $6 \mathrm{kHz}$, significant measurement errors in the magnitude output of a VT arise. The influence of the feeding (energisation) direction of the VT will also result in inaccuracies when the flow of energy is from the secondary side to the primary side of the VT, in this case the error starts at much lower frequencies (around $1 \mathrm{kHz}$ ). In a real network this scenario does not often occur but when it does there is often a fault on the network rendering the rms measurement sample invalid as per IEC 61000-4-30 flagging rules [49].

Figure 2-28 shows the useful frequency range (in magnitude) of current transformers for the different technologies deployed. The useful range of inductive Current Transformers (CTs) which are used by utilities can be observed, as frequencies of up to $50 \mathrm{kHz}$ can accurately be measured by this type of technology. While various applications exist for the various technologies, for the purpose of this thesis, the accuracy of inductive CTs is considered to be sufficient, as measurements will concentrate on lower order harmonics of below $1 \mathrm{kHz}$. 


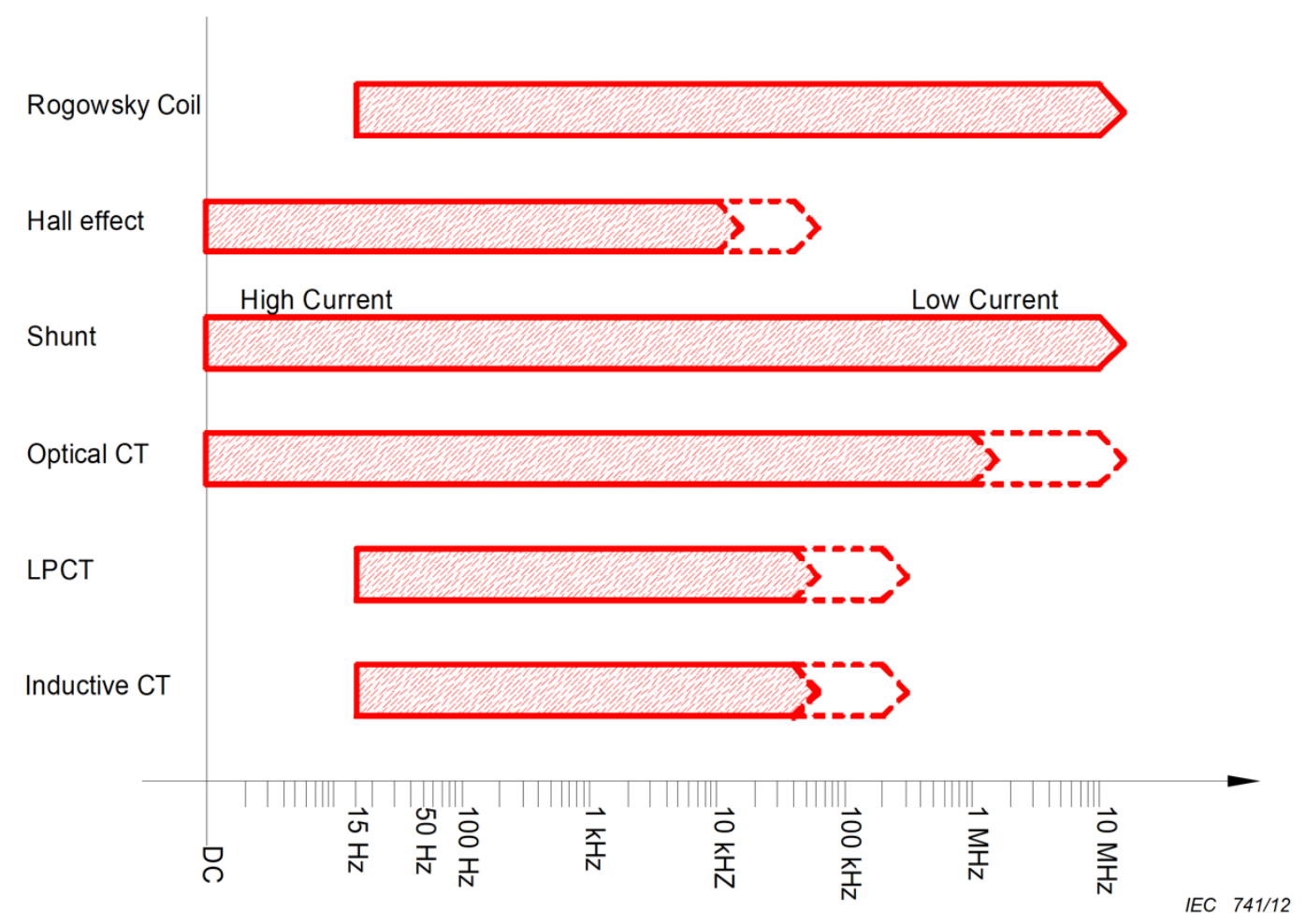

Figure 2-28. Current transformer technologies frequency range [87]

\subsubsection{Harmonic measurement circuit}

The measurement of harmonic distortion is not limited to the PQ instrument only, as the accuracy impact of the measurement circuit must also be considered when results are analysed.

Figure 2-29 shows a typical metering installation for billing and secondary measurements (on secondary side of instrument transformer) at utility level, often used to measure the PQ levels for RPPs and normal load customers. The circuit starts with HV VT and CT, which feed into junction boxes where cables are terminated and protection such as fuses are located.

These junction boxes are typically situated in the HV yard next to the VTs and CTs. Cable lengths from the VTs and CTs to the junction box are typically in the order of a few meters, however, the cable length from the junction box to the control panel in the control room may be in the order of a few 100 meters. These cables then connect onto the respective metering equipment where additional transducers may be needed to convert for example current to voltage at a PQ instrument. These long cable lengths results in a volt drop across the cable which influence the measurement input into the PQ recorder. Granted the voltage drop may be deemed as small but it must be taken into account for the measurement uncertainty. 


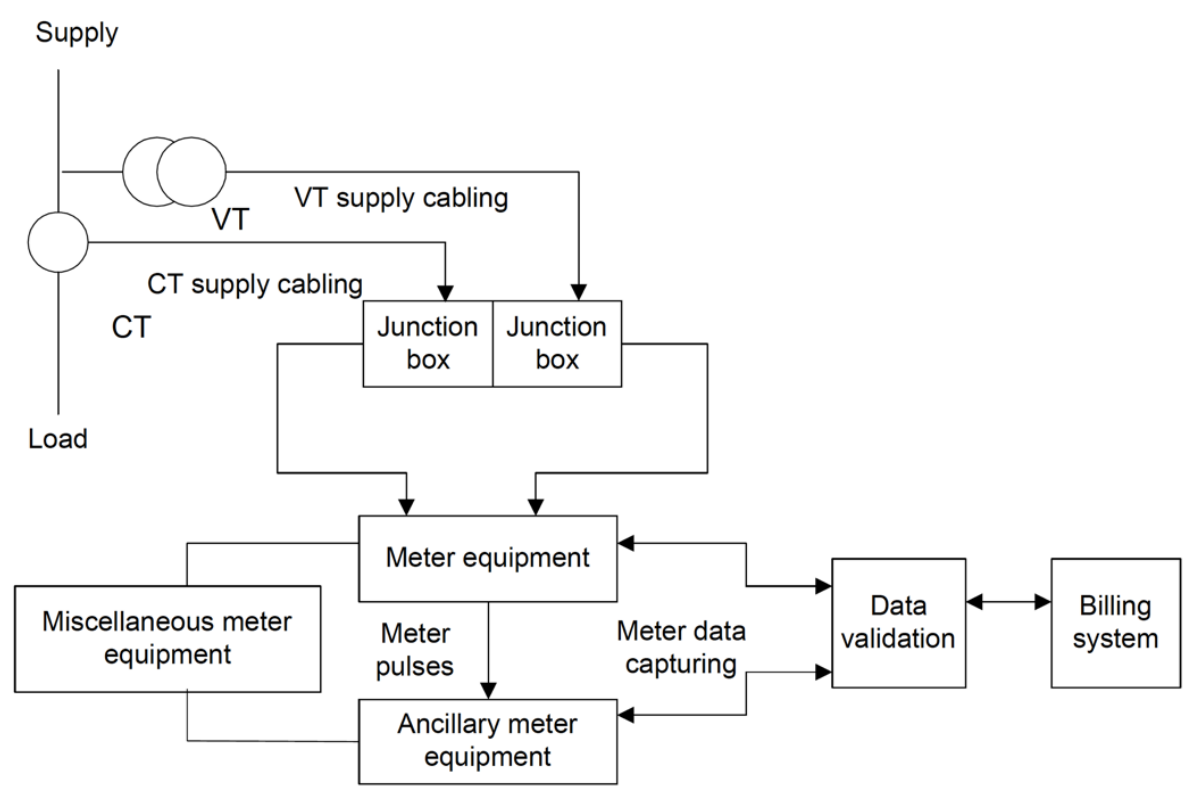

Figure 2-29. Typical metering system for billing and secondary measurements [88].

\subsection{Conclusion}

\section{The sources of harmonics}

Renewable energy sources are a known source of harmonic distortion, through the application of inverter-based technologies. Wind turbines, typically type 3 and type 4, make use of inverter technology in the generation of electrical energy. PV farms fully make use of inverter technologies in the energy conversion process.

RPP plants are often conduct a predictive harmonic emission assessment through the simulation of inverters based on the test sheet of inverters or generic models. These test sheets are based on pure sinewaves and it has been found that harmonic distortion output of inverters differ when background harmonic distortion exist in the inverter input signals.

It is needed that manufacturers test the harmonic emission of inverters under conditions reflecting the distorted grid voltages found in real networks, as ideal conditions result in optimistic harmonic emission performance figures.

Managing sources of harmonic distortion: utility perspective

As the owner of the network, electrical utilities have to manage harmonic distortion at any PCC, and they have the responsibility of serving voltage of sufficient quality (in this case voltage THD) to all users. For this reason, the extent by which users contribute to harmonic distortion, has to be contained in keeping the global impact to acceptable (planning) levels. This can be done by allocating a specific portion of the resulting total voltage THD to each user connected to a PCC.

An international standards document (IEC 61000-3-6 [4]) guides this procedure. Compliance to allocated harmonic emission limits is not easily proven due to the measurement problem, namely the lack of harmonic phasor data. This is the main research problem being addressed in this thesis. If litigation arises, the current state of knowledge is significantly deficient as no scientifically sound solution exists.

The IEC 61000-3-6 definition for emission makes use of the concept "emanates" and gives the reason why harmonic emission assessment at a network user should only take into account those 
harmonic currents emitted by that user only. Literature discussed in this chapter presented different solutions to assess (quantify and qualify) the direction and extent of harmonic distortion. All these methods have some deficiency as discussed in this chapter. How much harmonic emission is being emitted by, or absorbed into the upstream network? This remains the fundamental question to be addressed during harmonic emission assessment.

Measurement of harmonic emissions is estimated by engineering methods [15] or other approaches found in literature [15] by using either single-point measurements or multi-point measurements.

Single-point measurement requires accurate knowledge of the network impedance and of the load. Both network and load impedance can change over time and harmonic emission requires continuous assessment, as networks and loads are not always constant.

Multi-point measurements have to be done synchronously at all points of interest and with time stamping of sufficient accuracy, such as made possible by GPS systems in which a time uncertainty of better than $100 \mathrm{~ns}$ is relatively easy to obtain. Synchronising the recordings will ensure that each data sample is taken at exactly the same network conditions.

Application of the CIGRE/CIRED C4.109 method for harmonic emission assessment compares the (upstream) network harmonic voltage to the harmonic voltage at the PoC of a nonlinear load. Accounting for cause and consequence requires that both voltages have to be measured at the exact same time and at two different points in the network. At least two instruments are needed, and both have to be time synchronised from the same time source (i.e. GPS).

\section{Harmonic data analysis}

When measuring voltage and current harmonic phasors using an instrument that complies to the measurement requirements of the IEC 61000-4-30 Class A, edition 3, harmonic phasors are derived every 10/12 cycles of the voltage waveform. This results in an updated set of harmonic phasors every $200 \mathrm{~ms}$ (about, as each cycle in for example a $50 \mathrm{~Hz}$ power system is not necessarily $20 \mathrm{~ms}$ ). Having 63 voltage and current harmonic phasors every $200 \mathrm{~ms}$, result in 315 voltage and current harmonic phasors every second and in each phase (945 phasors). It is evident that after a day of accumulating ( $945 \times 60 \times 24$ ) voltage and current harmonic phasors and sending it to a remote point for analysis, using for example some form of Internet communication, result in a challenge of note when harmonic emission has to be tracked continuously.

The exception is the CIGRE/CIRED C4.109 scatterplot method which makes use of only 10-min rms values for voltage and current harmonic phasors.

Measurement uncertainty during harmonic emission assessment remains largely unknown and it is the most important deficiency currently during the assessment of grid code compliance. Limited experience and literature exist on how to assess measurement uncertainty at harmonic frequencies from a sound scientific point of view. Some literature exists on the performance of magnetic VTs and CTs, but the entire instrumentation chains contribute to measurement uncertainty.

Assessment of harmonic emission at a renewable energy source for the purpose of grid-code compliance is complex and requires a pragmatic solution, as literature indicates a number of deficiencies when a sound scientific consideration is applied.

Only those harmonic currents emitted by the RPP can be used during the assessment of grid-code compliance. Methods exist to determine the direction of harmonic distortion but require the continuous measurement of synchronised harmonic phasors at all points of interest and are not practical for most utilities.

The CIRED/CIGRE C4.109 scatterplot method seems to be a practical solution, but it lacks detailed interpretation to conclusively allocate harmonic emission to either the network (upstream) 
or the load (downstream). It was shown by computer simulation that this method can also lead to an incorrect assessment of the harmonic emission.

RPPs can be located anywhere in a Distribution or Transmission network, often with multiple RPPs connected onto the same busbar or node. Dynamic interaction of harmonic emission between RPPs and the electrical network occurs, and an innovative solution was not found in literature during the time of this research.

As grid code compliance is absolute, the measurement uncertainty of any method used in harmonic emission assessment must be well qualified and quantified. Limited publications address this important aspect of harmonic emission assessment . 


\section{CHAPTER 3: Verification of harmonic emission assessment methodologies}

\subsection{Introduction}

Harmonic emission assessment methodologies discussed in section 2.5 are verified by simulation of simplified, but representative, electrical circuits using a time-domain simulation approach. This is done to avoid simplifications needed when using frequency-domain equivalent models.

The Alternative Transients Program (ATP) is known for rigorous peer-review of models, resulting in continuous improvements. ATP is ideally suited for a time-domain simulation of power systems where transient conditions are important such as in the research on harmonic emission. For this reason, ATP is used to verify the circuit analysis via spreadsheet calculations.

Computer simulation allows a controlled experimental environment by having perfect control over variables. Changing only one variable allow causality between system response and changes in a specific variable.

Some methods are excluded from verification by computer simulation:

- Harmonic State Estimation [58] aims at wide area monitoring and requires simultaneous recording of harmonic distortion at different nodes. This method takes into account time dependant network operating conditions causing changes in harmonic distortion levels and not within scope of the computer simulation.

- The global power quality [59], [60] index is not considered as it evaluates voltage THD contributed by different feeders at a PCC. No specific harmonic order is evaluated, and the specific polluting load cannot be assessed for contractual harmonic emission. This is an important requirement of the research hypothesis.

- The RLC method [13], [14] assess harmonic impedance at a specific load from harmonic voltage and harmonic current phasors and cannot quantify harmonic emission assessment at a specific non-linear load.

Harmonic emission assessment methods that are included in computer simulation study:

- IEC 61000-3-6 [4] (Figure 2-14).

- Harmonic active power.

- CIGRE C4.109 (scatter plot method).

- Harmonic vector method.

- Discrimination of harmonic current phasors that

- Range of phase angles that result in secondary cancellation of harmonic phasors.

\subsection{Harmonic emission}

Circuit analysis is performed on Figure 2-14 with the aim of deriving the harmonic current $\boldsymbol{I}_{h}$, harmonic voltage $\boldsymbol{V}_{h}$, and harmonic emission $\boldsymbol{E}_{h c}$. The direction of $\boldsymbol{I}_{h}$ is defined towards the load as if supplied by the upstream network.

$$
\boldsymbol{I}_{h}=\frac{\boldsymbol{E}_{h 0}-\boldsymbol{I}_{h c} \boldsymbol{Z}_{h c}}{\boldsymbol{Z}_{h}+\boldsymbol{Z}_{h c}}
$$

The harmonic voltage phasor $\boldsymbol{V}_{h}$ is derived in (29) below.

$$
\boldsymbol{V}_{h}=\boldsymbol{Z}_{h c}\left(\boldsymbol{I}_{h c}+\boldsymbol{I}_{h}\right)
$$


Equation (30) calculates the voltage harmonic emission phasor $\boldsymbol{E}_{h c}$ from the network harmonic impedance $\boldsymbol{Z}_{h}$ and the derived harmonic current $\boldsymbol{I}_{h}$.

$$
\boldsymbol{E}_{h c}=\boldsymbol{Z}_{h} \boldsymbol{I}_{h}
$$

Simulation conditions selected (note the selected values are not based on a real network):

$\boldsymbol{E}_{h 0}=200 \angle 30^{\circ} \mathrm{V}$ at harmonic $h$ (all harmonic orders will use the same voltage value unless otherwise stated to ensure consistency.)

$R_{h}=R_{h c}=30 \Omega$

$L_{h}=6.3662 \mathrm{mH}$

$L_{h c}=318.30988 \mathrm{mH}$

Harmonic current phasors $\boldsymbol{I}_{h c}$ was varied as listed in Table 3-1 keeping other parameters fixed. By changing the input harmonic current $\boldsymbol{I}_{h c}$ it simulates the condition when the harmonic source is located in the load side.

Table 3-1. Values selected for parameter $\boldsymbol{I}_{h c}$

\begin{tabular}{|c|c|}
\hline \multicolumn{2}{|c|}{$\boldsymbol{I}_{\boldsymbol{h c}}$} \\
\hline Mag $[\boldsymbol{A}]$ & Angle $\left.{ }^{\circ}\right]$ \\
\hline 0.2 & 0 \\
\hline 0.6 & 0 \\
\hline 1 & 0 \\
\hline 1 & 30 \\
\hline 1.2 & 30 \\
\hline 1.4 & 30 \\
\hline 0.2 & 60 \\
\hline 0.4 & 60 \\
\hline 0.6 & 60 \\
\hline
\end{tabular}

Verification of calculated results through comparison of ATP model

By considering only the $5^{\text {th }}$ harmonic, the simulated results were verified through calculations.

Calculated results for $\boldsymbol{I}_{h}, \boldsymbol{V}_{h}$, and $\boldsymbol{E}_{h c}$ are compared in Table 3-2 to Table 3-4 to the simulated results. The time-domain simulation confirms the fundamental circuit analysis.

Table 3-2 Calculated results compared to simulated results for $\boldsymbol{I}_{h}$

\begin{tabular}{|c|c|c|c|c|c|}
\hline \multicolumn{2}{|c|}{ Input Ihc } & \multicolumn{2}{|c|}{ Ih calculated } & \multicolumn{2}{|c|}{ Ih simulated } \\
\hline $\operatorname{Mag}[A]$ & Angle $\left.1^{\circ}\right]$ & Mag [V] & Angle [ ${ }^{\circ}$ & Mag [V] & Angle [ $\left.{ }^{\circ}\right]$ \\
\hline 0.2 & 0.0 & 0.3 & -83.3 & 0.3 & -82.4 \\
\hline 0.6 & 0.0 & 0.5 & -135.5 & 0.5 & -134.6 \\
\hline 1.0 & 0.0 & 0.8 & -153.6 & 0.8 & -152.7 \\
\hline 1.0 & 30.0 & 1.0 & -124.5 & 1.0 & -123.6 \\
\hline 1.2 & 30.0 & 1.2 & -128.0 & 1.2 & -127.1 \\
\hline 1.4 & 30.0 & 1.4 & -130.6 & 1.4 & -129.6 \\
\hline 0.2 & 60.0 & 0.5 & -73.4 & 0.5 & -72.5 \\
\hline
\end{tabular}




\begin{tabular}{|c|c|c|c|c|c|}
\hline \multicolumn{2}{|c|}{ Input Ihc } & \multicolumn{2}{|c|}{ Ih calculated } & \multicolumn{2}{|c|}{ Ih simulated } \\
\hline $\operatorname{Mag}[A]$ & Angle $[\circ]$ & Mag [V] & Angle $[\circ]$ & Mag [V] & Angle $\left[{ }^{\circ}\right]$ \\
\hline 0.4 & 60.0 & 0.7 & -85.0 & 0.7 & -84.2 \\
\hline 0.6 & 60.0 & 0.8 & -92.1 & 0.8 & -91.2 \\
\hline
\end{tabular}

Table 3-3 Calculated results against simulated results for $\boldsymbol{V}_{h}$

\begin{tabular}{|c|c|c|c|c|c|}
\hline \multicolumn{2}{|c|}{ Input $I_{h c}$} & \multicolumn{2}{|c|}{$V_{h}$ calculated } & \multicolumn{2}{|c|}{$V_{h}$ simulated } \\
\hline $\operatorname{Mag}[A]$ & Angle $\left[{ }^{\circ}\right]$ & Mag [V] & Angle $\left[{ }^{\circ}\right]$ & Mag [V] & Angle $\left[^{\circ}\right]$ \\
\hline 0.2 & 0.0 & 201.1 & 32.9 & 201.1 & 33.9 \\
\hline 0.6 & 0.0 & 213.3 & 32.3 & 213.2 & 33.2 \\
\hline 1.0 & 0.0 & 225.4 & 31.7 & 225.3 & 32.6 \\
\hline 1.0 & 30.0 & 224.6 & 35.8 & 224.5 & 36.7 \\
\hline 1.2 & 30.0 & 230.5 & 36.2 & 230.5 & 37.1 \\
\hline 1.4 & 30.0 & 236.5 & 36.6 & 236.4 & 37.5 \\
\hline 0.2 & 60.0 & 199.2 & 34.6 & 199.2 & 35.5 \\
\hline 0.4 & 60.0 & 203.5 & 35.9 & 203.4 & 36.8 \\
\hline 0.6 & 60.0 & 207.8 & 37.1 & 207.8 & 38.0 \\
\hline
\end{tabular}

Table 3-4 Calculated results against simulated results for $\boldsymbol{E}_{h c}$

\begin{tabular}{|c|c|c|c|c|c|}
\hline \multicolumn{2}{|c|}{ Input $I_{h c}$} & \multicolumn{2}{|c|}{$E_{h c}$ calculated } & \multicolumn{2}{|c|}{$E_{h c}$ simulated } \\
\hline $\operatorname{Mag}[A]$ & Angle $\left[{ }^{\circ}\right]$ & Mag [V] & Angle $\left[{ }^{\circ}\right]$ & Mag [V] & Angle $\left[{ }^{\circ}\right]$ \\
\hline 0.2 & 0.0 & 10.3 & -64.9 & 10.3 & -64.0 \\
\hline 0.6 & 0.0 & 15.6 & -117.0 & 15.6 & -116.2 \\
\hline 1.0 & 0.0 & 26.2 & -135.2 & 26.2 & -134.2 \\
\hline 1.0 & 30.0 & 32.5 & -106.1 & 32.5 & -105.2 \\
\hline 1.2 & 30.0 & 38.3 & -109.6 & 38.3 & -108.7 \\
\hline 1.4 & 30.0 & 44.2 & -112.1 & 44.2 & -111.2 \\
\hline 0.2 & 60.0 & 16.1 & -55.0 & 16.0 & -54.0 \\
\hline 0.4 & 60.0 & 21.0 & -66.6 & 21.0 & -65.7 \\
\hline 0.6 & 60.0 & 26.4 & -73.7 & 26.4 & -72.8 \\
\hline
\end{tabular}

With the calculations values or circuit analysis verified through simulations, the calculated results will be used throughout this chapter unless otherwise stated for the various methods applied. Changes are easier implemented through the calculations then through the simulations hence the use of calculated results.

\section{Visualisation of voltage harmonic emission}

Figure 3-1 displays the $5^{\text {th }}$ harmonic voltage emission as per IEC 61000-3-6 discussed in section 2.2. It is based on the results listed in Table 3-2 to Table 3-4. The diagram shows the summation of the harmonic voltage phasors $\boldsymbol{E}_{h 0}, \boldsymbol{V}_{h}$ and $\boldsymbol{E}_{h c}$ in a loop analysis of Figure 2-14, where the harmonic voltage at the network $\boldsymbol{E}_{h 0}$, the harmonic voltage drop $\boldsymbol{E}_{h c}$ and the load harmonic voltage $\boldsymbol{V}_{h}$ all summate to zero volt as per Kirchhoff's Voltage Law [89]. 


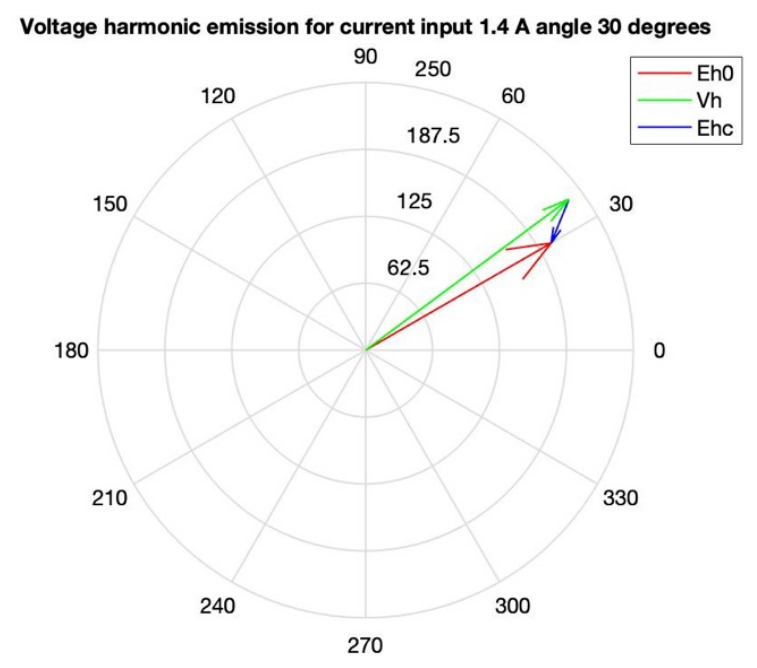

Figure 3-1. Voltage harmonic emission diagram for harmonic current input $1.4 \angle 30^{\circ}$ A for the $5^{\text {th }}$ harmonic

With the concept of harmonic emission verified through simulations, the assessment of harmonic emission is presented next.

\subsection{Harmonic emission assessment using harmonic active power with one source of harmonic distortion}

Harmonic active power to determine the direction of harmonic distortion is evaluated by computer simulation in this section

Complex power is calculated for the $5^{\text {th }}$ harmonic and shown in Figure 3-2 and Figure 3-3. Fundamental frequency active power is from the upstream network into the load.

Only one harmonic source exist and is located within the load. $5^{\text {th }}$ Harmonic complex power in Figure 3-2 is located in the $3^{\text {rd }}$ quadrant, confirming that harmonic active power is opposite in direction to fundamental frequency active power and why harmonic active power, under the assumptions used in Figure 2-9, can be considered as a parameter to be used in both the assessment and localisation of the source of harmonic distortion.

It is expected that if the harmonic source is located in the upstream network, that the same conclusion that achieved above, will apply. $5^{\text {th }}$ Harmonic complex power now concentrates in quadrant 4 as shown by Figure 3-3. Harmonic active power and fundamental frequency active power now have the same sign (direction) indicating that the harmonic active power can be used to localise the source of harmonic distortion when there exist only one source of harmonic distortion in the network under consideration. 


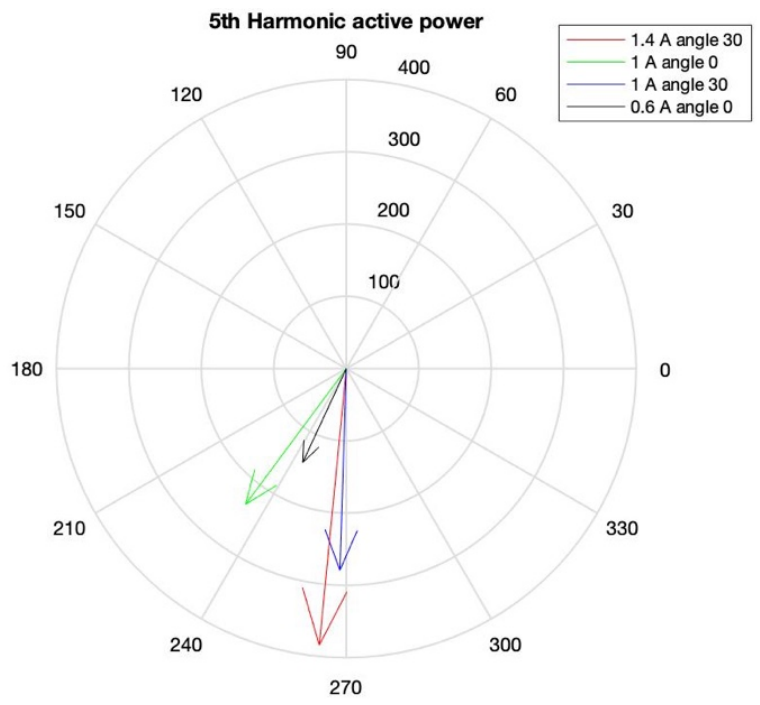

Figure 3-2. Complex harmonic active power with only load as a harmonic source

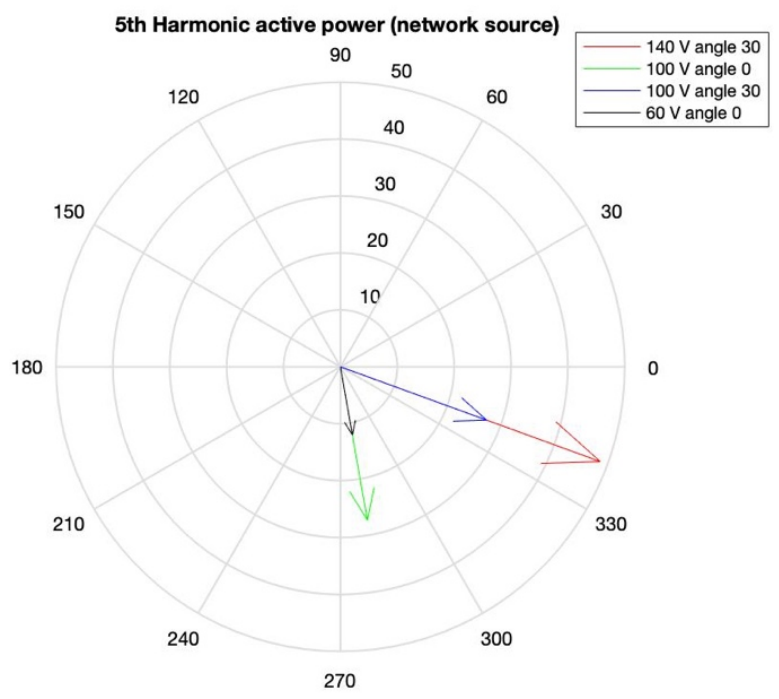

Figure 3-3. Complex harmonic active power with only network as a harmonic source

Results obtained above confirms that the useless (imaginary or reactive) power component cannot be used to better understand the contribution to harmonic distortion by a distorting (non-linear) source in a network. The reactive harmonic power component remain near-orthogonal to the active power, resulting in limited opportunity to distinguish with sufficient certainty on the location of the harmonic source being upstream or downstream.

\subsection{Harmonic emission assessment using harmonic active power with more than source of harmonic distortion}

In real networks non-linear loads are distributed all over the network. A PCC interconnects more than one source of distortion in a real network. Harmonic emission assessment is now evaluated using two thyristor controlled rectifiers.

From the previous section, it was found that the sign of harmonic active power will be opposite to the sign of the active power in the fundamental frequency component and can therefore be used to evaluate both the localisation and contribution of harmonic active power. This result is now tested for the case when the more than one harmonic distortion source exists.

When only one source of harmonic emission in a network exist, it follows that harmonic emission are from the single nonlinear load and the only cause to the voltage waveform distortion in $\mathrm{V}_{\text {PCC }}$ at the Point of Common Coupling (PCC) as indicated in Figure 3-4. How much that nonlinear load contributes can be assessed by measuring in the feeder connecting the rectifier to the PCC.

Rectifiers [90] are known sources of harmonic distortion and modelled using ATP as shown in Figure 3-4. Two 6-pulse thyristor controlled rectifiers [53] are energised from a Thévenin equivalent $22 \mathrm{kV}$ distribution network. No background harmonic sources exist in the network.

Harmonic distortion at the PCC is only from the non-linear loads. The goal is to assess how much each contributes. Supply network parameters are similar to a real-life $22 \mathrm{kV}$ distribution network.

Being a computer time-domain simulation result in a perfect time-synchronisation of measurements obtained at each of the 3 points of interest in this circuit, Load 1, Load 2 and the incoming feeder from the supply network. The principle by which ATP simulate the circuit, emulates a real-life measurement system as ATP solve circuit equations during each time-step, 
using the solutions obtained during the previous time-step, as input values solving the variables during the next time-step.

The output of the ATP simulation is a set of waveforms and because the fundamental frequency is perfectly stable, any single but complete $20 \mathrm{~ms}$ ( $50 \mathrm{~Hz}$ fundamental frequency chosen) set of 3phase voltage and current waveforms can be used during the Fourier analysis. It is important to not include for example the first few cycles. ATP does include the initial network conditions and by a simple visual inspection, it is needed to select subsequent cycles with no sign of any transient conditions (i.e. charging the capacitors cannot be immediate).

Fourier analysis requirements have to be respected to avoid spectral leakage for example. The simplest is to set the simulation to produce $2^{n}$ datapoints that are perfectly aligned to subsequent zero crosses of the $50 \mathrm{~Hz}$ reference waveform and/or consider a version of the digital Fourier transform that is not sensitive to the number and alignment of datapoints.

As a first evaluation, by keeping the thyristor firing angles at $20^{\circ}$ at both Load 1 and Load 2, they emulate a "single" source of harmonic emission because each will inject the same amount of harmonic distortion (both have the exact same DC load connected). Figure 3-5 visualise the $5^{\text {th }}$ harmonic active power component of the complex harmonic power results for the various firing angles at Load 2, including when both firing angles of Load 1 and Load 2 are kept at $20^{\circ}$. Harmonic active power from the two non-linear loads are the same magnitude and phase angle, which double (in magnitude) the total complex power into the network.

Figure 3-6 shows the harmonic active power for the $7^{\text {th }}$ harmonic.

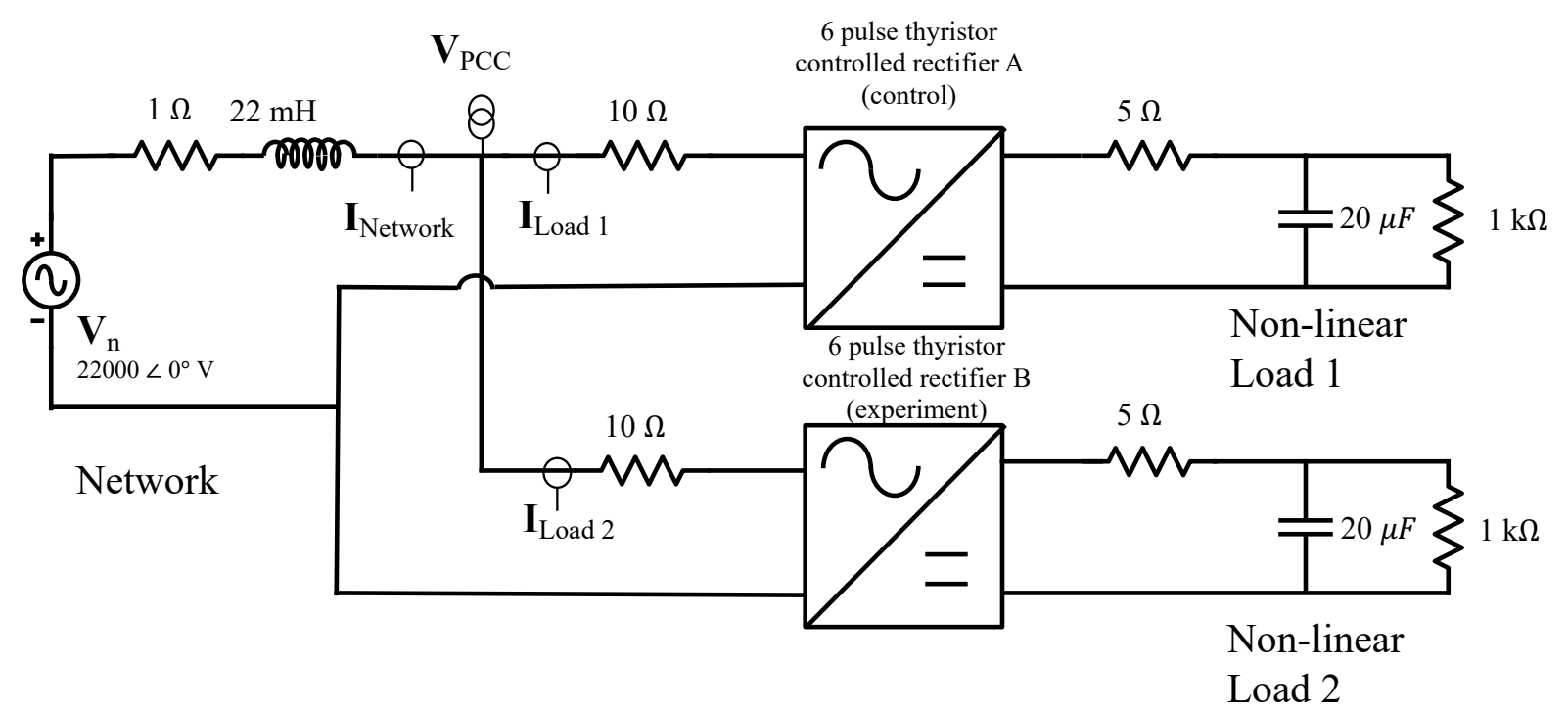

Figure 3-4. Thyristor controlled circuit used for simulation

A second evaluation is now done to simulate the conditions in a real network where two different nonlinear loads are operated independent from each other. The firing angle at Load 1 is kept fixed at $20^{\circ}$ whilst the firing angle of Load 2 is now incremented by $20^{\circ}$ starting at $20^{\circ}$ until $120^{\circ}$. The passive load elements in the DC circuit of each are kept fixed.

As the firing angle of Load 2 is increased above $40^{\circ}$ the direction of the harmonic active power from Load 1 and Load 2 changes as shown in Figure 3-5 and Figure 3-6 for the $5^{\text {th }}$ and $7^{\text {th }}$ harmonic orders respectively. Above $80^{\circ}$ firing angle the sign of the harmonic active power from the two loads are opposite in sign when compared to each other, indicating that the direction of harmonic active power from the two loads are not in the same direction when compared to the $20^{\circ}$ firing angle. 
$5^{\text {th }}$ harmonic active power

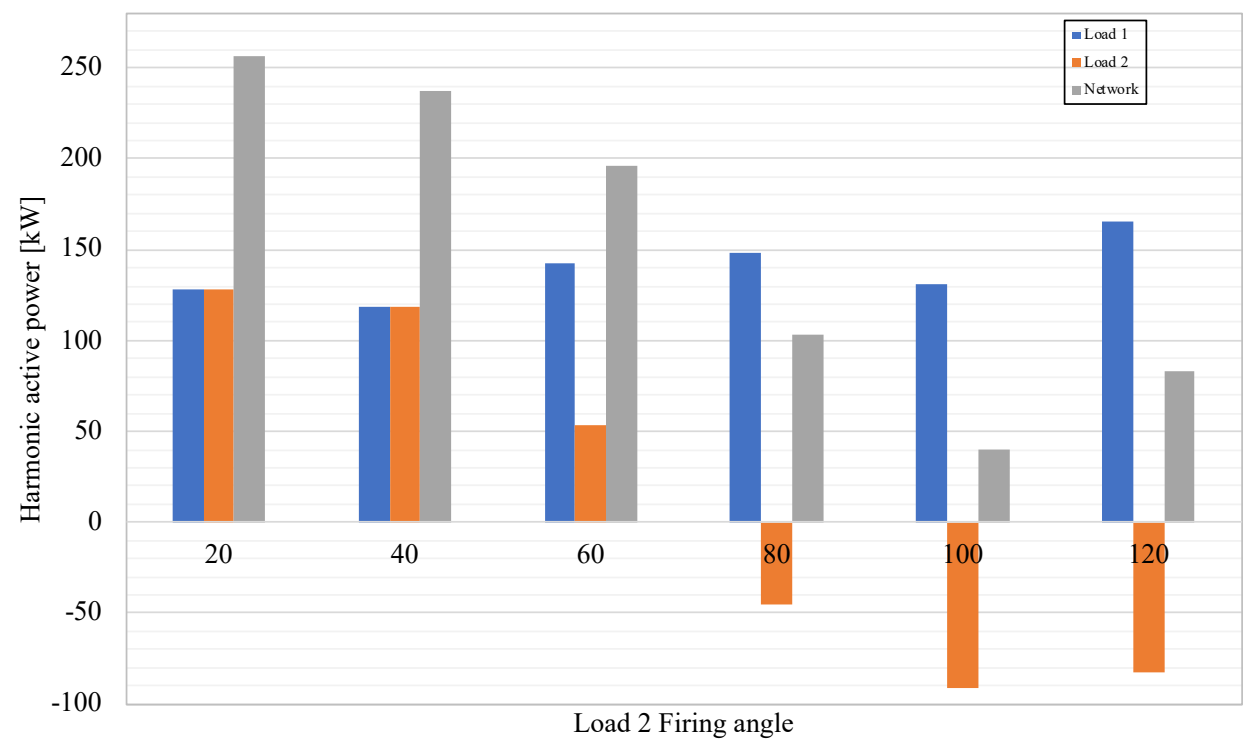

Figure 3-5 $5^{\text {th }}$ harmonic active power for various firing angles of Load 2

$7^{\text {th }}$ harmonic active power

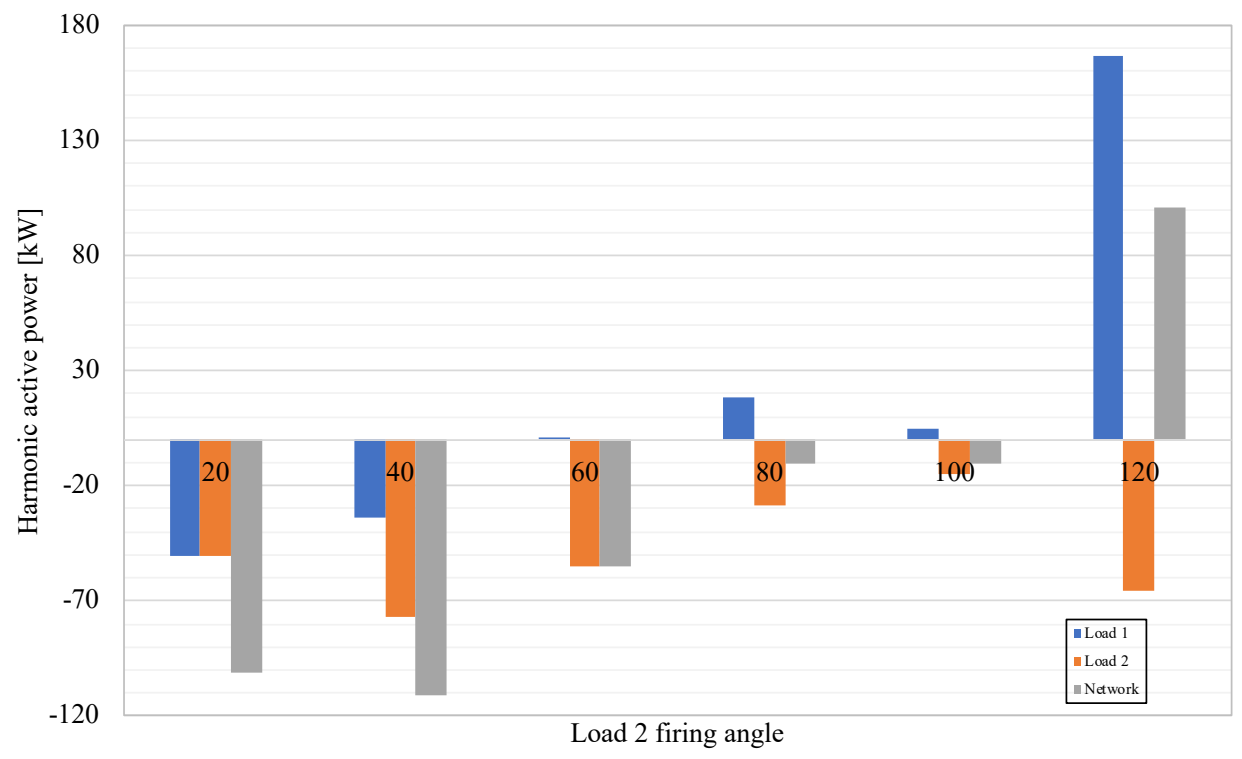

Figure 3-6. $7^{\text {th }}$ harmonic active power for various firing angles of Load 2

Although the principles in this section were only presented for the $5^{\text {th }}$ and $7^{\text {th }}$ harmonic component, similar results are obtained when the total harmonic active power is considered ([16], [52]). The focus in this section is to emphasise the principle of harmonic active power exchange between different nonlinear loads and when considered from a single harmonic point of view, the simplest to understand.

The extent to which harmonic active power flows in an opposite direction to the fundamental frequency active power can be seen in the sum of the harmonic active power as observed in the incoming feeder. For the $5^{\text {th }}$ harmonic shown in Figure 3-5 at $100^{\circ}$ firing angle, the harmonic active power into the supply network is drastically reduced when compared to the case for a $20^{\circ}$ firing angle. This is due to the harmonic active power from the two non-linear loads having an opposite sign. 
Results for the $7^{\text {th }}$ harmonic in Figure 3-6 are similar.

\section{Harmonic active power in the assessment harmonic emission}

An advantage of this approach is that information on the load or network harmonic impedance is not required. Field measurements could, seemingly obtained with relative ease. It is important to carefully consider the metrological requirements of gaining any useful results when monitoring harmonic active power in a real distribution network.

Accurate, and synchronous measurement of both harmonic voltage and harmonic current phasors are required between the different input channels of an instrument to ensure that no phase offset occurs between different voltage or current input channels, or between a phase voltage and a current input channel. Because the inductive impedance increase with harmonic number, a small phase offset between a voltage and current harmonic phasor, can result in an opposite sign for harmonic active power as the current harmonic phasors becomes increasingly orthogonal with the voltage harmonic phasor.

Frequency response of voltage and current instrumentation requires careful consideration as an error in phase angle measurement when calculating harmonic active power in (3) can also result in a change of the sign. Measurement uncertainty needs to be qualified and quantified as the measurement chain with increasing harmonic numbers, are increasingly compromised by the challenge of having a measurand within an acceptable measurement uncertainty.

Practical application of harmonic active power using measurements in real distribution networks with the intent for interpretation against harmonic emission, can hardly be realised to serve any useful results to grid code compliance assessment.

In a real network, harmonic distortion sources are distributed all over. Harmonic distortion in any feeder connected to a PCC will reflect the interaction of the different nonlinear loads at that moment in time. Consequences to harmonic emission are:

- Harmonic active power in any single feeder merely reflects the nett result of how different nonlinear loads interact at that moment. It has no value towards interpreting harmonic emission contributed by that specific nonlinear load.

- Harmonic active power is not absolute, it has to evaluated continuously to include the changes in network operating states. This requires continuous monitoring to reflects the dynamic interaction between different loads.

- Measurements of harmonic voltage and current phasors have to be taken at all points in the network and in perfect synchronism, i.e. in all feeders out of, and into, the PCC.

Harmonic active power in the assessment of harmonic emission in a real network result in complex and stringent requirements on the measurement system, possible out of scope to the average engineer involved in daily network operations.

\section{Considerations on deriving harmonic active power from field data}

A valuable relation to the real-world implementation of harmonic emission assessment in general (not only applicable to the analysis of harmonic active power) is realised by the time-domain simulation. In a digital measurement system, the number of datapoints and the resolution of the datapoints are normally certified as being compliant to the IEC 61000-4-30 measurement requirements up to for example the $63^{\text {rd }}$ harmonic component. Detection of zero crosses and the number of datapoints in a power system where the fundamental frequency is not perfectly stable, are also stipulated this PQ measurement standard. Of interest to field data used in this research, is the Class A measurement requirements of Edition 3, IEC 61000-4-30 ([49]). 
Time-synchronisation precision is not specified in the IEC 61000-4-30 Edition 3 measurement standard to be beyond a $1 / 2$-cycle time certainty. Using an UTC time-source "such as GPS" is merely mentioned, not stipulated, but important to obtain during field measurements, coherent network data in the true sense of the word.

Using GPS, it is only possible to achieve a time-uncertainty of $100 \mathrm{~ns}$ in time-stamping measurements obtained geographically separated from each other. When more than one instrument is needed, perfect coherent data is a fundamental requirement and not considered in an ATP simulation as the simulation process takes care of it. It can be expected that the constraints noted from the measurement results will be even more pronounced when using field data.

\subsection{Harmonic emission assessment by the CIGRE C4.109 scatter plot method}

Harmonic voltage and current phasors $\boldsymbol{V}_{h}$ and $\boldsymbol{I}_{h}$ as obtained by the ATP simulation of Figure 2-14 (as used in section 3.2 and section 3.3) is again used to evaluate the CIGRE C4.109 scatter plot assessment of harmonic emission. Different cases are considered: when;

- Harmonic source only exists in the load ( $\boldsymbol{I}_{h}$ is varied to emulate the harmonic source),

- Harmonic source only exists the upstream network ( $\boldsymbol{E}_{h 0}$ is varied),

- Harmonic source exists in both the upstream network and load (both $\boldsymbol{I}_{h}$ and $\boldsymbol{E}_{h 0}$ are varied).

The latter case represents the real-world condition whilst the first two cases considers how each scenario on its own impact harmonic emission. Only the $5^{\text {th }}$ harmonic order is evaluated as other harmonic orders are similar.

Figure 3-7 presents a CIGRE C4.109 scatter plot for when the harmonic source exists only in the load, no harmonic voltages in the upstream network ( $\boldsymbol{E}_{h 0}$ is zero). Observe that all data points are located exactly on the network impedance locus as predicted by the CIGRE C4.109 assessment principle.

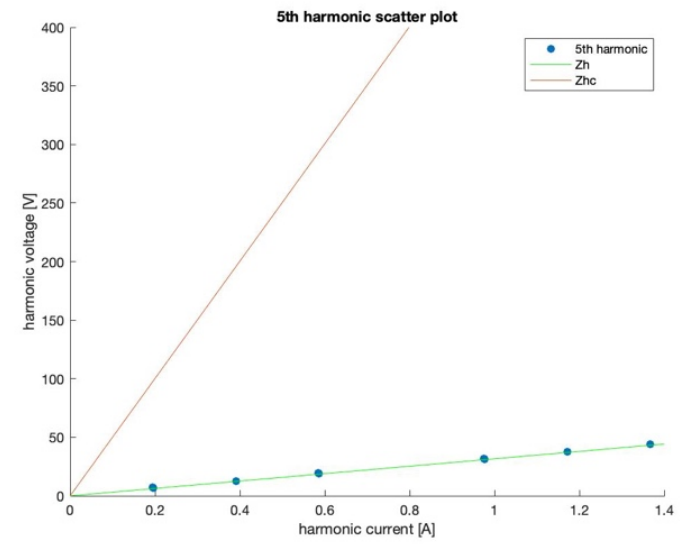

Figure 3-7. CIGRE C4.109 scatterplot when no harmonic distortion exist in upstream network

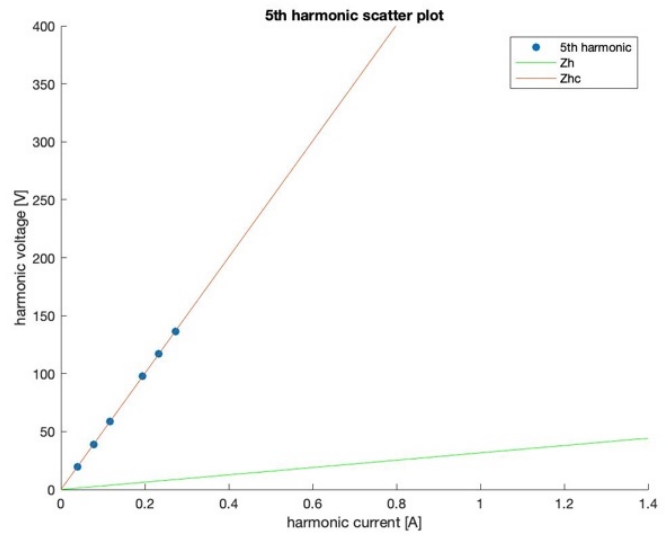

Figure 3-8. CIGRE C4.109 scatterplot when no harmonic distortion exist in the load

Figure 3-8 presents the CIGRE C4.109 results when the harmonic source is located in the upstream network. Again, all data points align perfectly with the load impedance locus as predicted.

$5^{\text {th }}$ harmonic phasor values listed in Table 3-5 are used as input values into the simulation of Figure 2-14 to represent the case when a harmonic source exist in both the upstream network and the load to simulate a real network. Both harmonic sources act on the PoC simultaneously further complicating the assessment of harmonic emission, as the challenge is to determine which harmonic source is the dominant emitter towards the harmonic distortion at the PoC. 
Table 3-5. Inputs used when harmonic source is in both the network and the load

\begin{tabular}{|c|c|c|c|}
\hline \multicolumn{2}{|c|}{ Input Eho } & \multicolumn{2}{c|}{ Input Ihc } \\
\hline Mag [V] & Angle [ ${ }^{\circ}$ & Mag $[\boldsymbol{A}]$ & Angle $\left[{ }^{\circ}\right.$ ] \\
\hline 20 & 30 & 0.2 & 0 \\
\hline 100 & 0 & 0.6 & 0 \\
\hline 250 & 0 & 1 & 0 \\
\hline 170 & 30 & 1 & 30 \\
\hline 300 & 30 & 1.2 & 30 \\
\hline 400 & 60 & 1.4 & 30 \\
\hline 30 & 90 & 0.2 & 60 \\
\hline 160 & 0 & 0.4 & 60 \\
\hline 180 & 0 & 0.6 & 60 \\
\hline
\end{tabular}

Results for the case when the harmonic source exist in both the network and the load is presented in Figure 3-9.

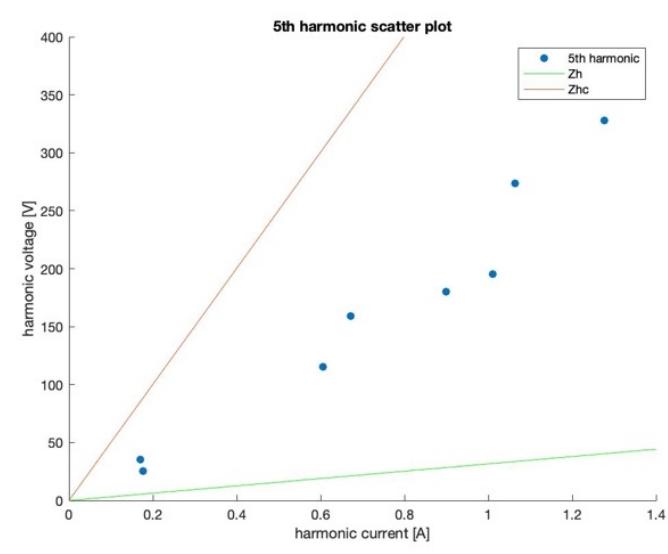

Figure 3-9. CIGRE C4.109 scatterplot when harmonic sources exist in the upstream network and load side

Figure 3-9 shows that the scatter plot method shows that both parties contribute towards the harmonic distortion but does not discriminate the harmonic values which may be deemed to be emission and which not, this is due to qualitative nature of this harmonic emission assessment method. In comparison the complex power assessment method can discriminate between harmonic emission that are emitted against those being absorbed.

\section{The value in the CIGRE C4.109 method}

Only the harmonic voltage and harmonic current rms values have to be recorded. No phase angle information is needed. Accurate time-synchronisation between input channels of the instrument or between different instruments are not needed as aggregate 10-min rms values are used. The instrument do not have to be of the Class A metrological requirements of IEC 61000-4-30 edition 3 as the method is fundamentally qualitative and not that much quantitative.

It is important that harmonic impedance of the loading- and upstream network is known. This in itself could be a challenge in practical cases. From a network simulation, it is possible that the electrical utility can fairly accurate predict the harmonic impedance profile of the supply network, but to what extent an IPP for example, will be able to provide an accurate harmonic impedance profile of the renewable energy plant, and under all possible contingencies, are not known. 
Interpretation of the results is fairly simple as shown above:

- All the harmonic emission is from the load,

- All the harmonic emission is from the supply network,

- Both the load and the supply network contribute to voltage waveform distortion.

The latter is what possibly be mostly encountered during field studies. With the datapoints concentrating towards one of the impedance loci, the "most dominant" source of voltage waveform distortion can be identified. It remains a qualitative assessment as the exact contribution by each, cannot be assessed by the CIGRE C4.109 method.

A possible improvement to this method is in discriminating between the harmonic voltage values resulting from the injection of harmonic current (harmonic voltage increase) and those harmonic voltages that has decreased because the load has absorbed harmonic current (similar to being sunk by a harmonic filter). Doing this, it is postulated and validated later in this thesis, the CIGRE C4.109 approach can then be used to improve the quantitative assessment performance. Two different scatterplots are then obtained that better visualise the relative contribution to waveform distortion by each.

\subsection{Harmonic emission assessment by the Harmonic Vector Method}

A harmonic phasor is preferred to the term "vector" as having a magnitude and phase angle is seemingly similar to what is in general referred to as a "vector" (a force can also have magnitude and 'angle' - direction). By application of the Fourier transform, harmonic phasors are obtained. Harmonic phasors are used in the frequency domain to better understand and analyse time domain phenomena [91]. Where possible, the concept of "harmonic vectors" will be avoided in this thesis.

The Harmonic Vector Method (HVM) aims to distinguish between the contribution by the loading network and the contribution by the upstream network to the measured harmonic current phasor $\boldsymbol{I}_{h}$. A Norton equivalent of Figure 2-14 is shown in Figure 3-10 as need to apply the HVM.

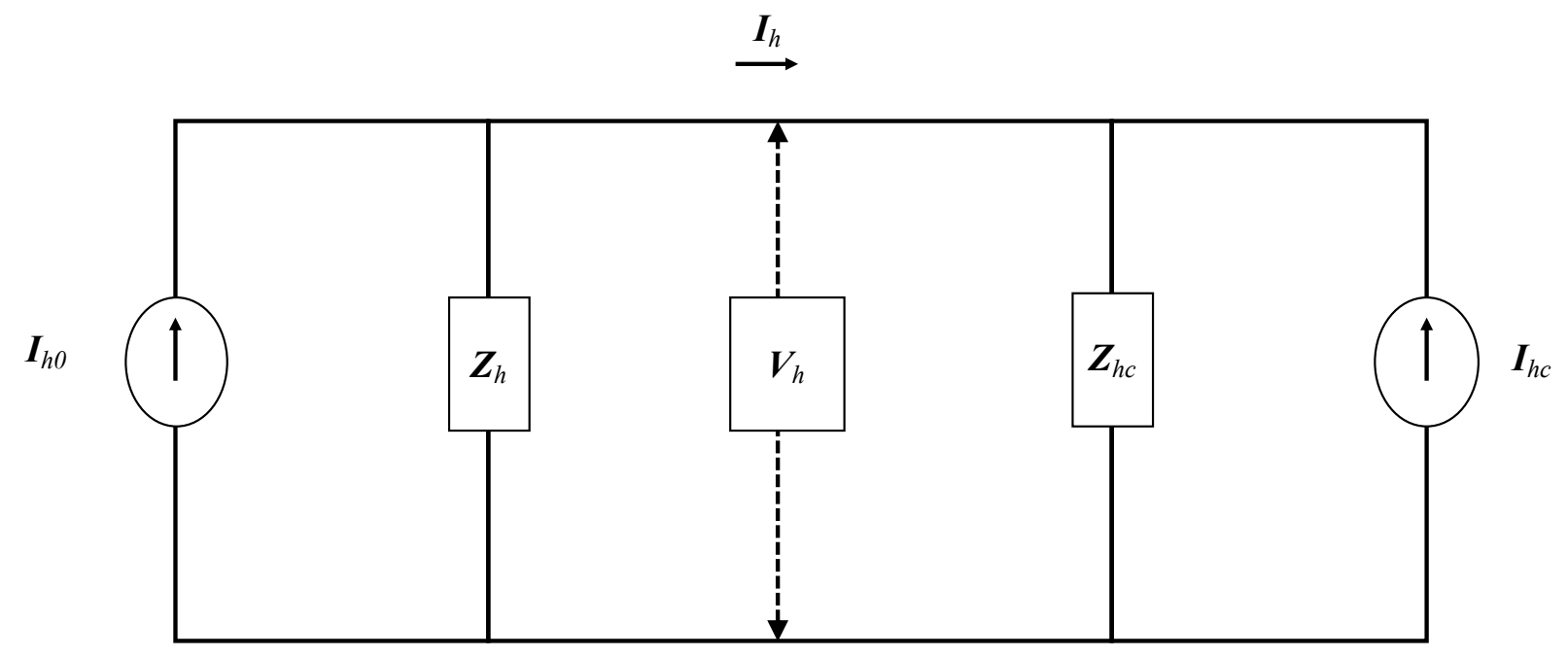

Figure 3-10. Norton equivalent circuit diagram used to simulate the HVM.

Equations (8) and (9) can be rewritten making used of the variables shown Figure 3-10.

$$
\boldsymbol{I}_{h 0}=\frac{\boldsymbol{V}_{h}}{\boldsymbol{Z}_{h}}+\boldsymbol{I}_{h}
$$




$$
\boldsymbol{I}_{h c}=\frac{\boldsymbol{V}_{h}}{\boldsymbol{Z}_{h c}}-\boldsymbol{I}_{h}
$$

In a real network harmonic voltage and current measurements $\left(\boldsymbol{I}_{h}\right.$ and $\left.\boldsymbol{V}_{h}\right)$ will typically be taken at a $\mathrm{PoC}$ of a non-linear load. Harmonic distortion contributions by the upstream network and the Apportionment of the measured harmonic distortion between to the upstream network and the nonlinear load is the goal of the harmonic vector method. This apportionment is achieved through the application of (31) and (32) to result in harmonic current phasors $\boldsymbol{I}_{h 0}$ and $\boldsymbol{I}_{h c}$ to represent the upstream harmonic current contribution and the load contribution, respectively.

In the next section manual calculations are undertaken, which are then verified through a simulation of Figure 3-10 in ATP.

\section{Model Verification}

Manual calculations of $\boldsymbol{I}_{h 0}$ and $\boldsymbol{I}_{h c}$ are done using equations (31) and (32) with input variables $\boldsymbol{I}_{h}$ and $\boldsymbol{V}_{h}$ taken from Table 3-2 and Table 3-3. Results of the manual calculations are listed in Table 3-6.

Table 3-6. Calculation results for $\boldsymbol{I}_{h 0}$ and $\mathbf{I}_{h c}$ in application of HVM.

\begin{tabular}{|c|c|c|c|c|c|}
\hline \multicolumn{2}{|c|}{ Input values } & \multicolumn{4}{c|}{ Calculated Results } \\
\hline I $\boldsymbol{I}_{h}$ & \multicolumn{2}{c|}{$\boldsymbol{I}_{h c}$} & \multicolumn{2}{c|}{$\boldsymbol{I}_{h 0}$} \\
\hline 0.3 & Angle [\%] & Mag [A] & Angle [ ${ }^{\circ}$ ] & Mag [A] & Angle [ ${ }^{\circ}$ ] \\
\hline 0.5 & -83.3 & 0.2 & 0.0 & 6.3 & 11.6 \\
\hline 0.8 & -135.5 & 0.6 & 0.0 & 6.3 & 11.6 \\
\hline 1.0 & -153.6 & 1.0 & 0.0 & 6.3 & 11.6 \\
\hline 1.2 & -124.5 & 1.0 & 30.0 & 6.3 & 11.6 \\
\hline 1.4 & -128.0 & 1.2 & 30.0 & 6.3 & 11.6 \\
\hline 0.5 & -130.6 & 1.4 & 30.0 & 6.3 & 11.6 \\
\hline 0.7 & -73.4 & 0.2 & 60.0 & 6.3 & 11.6 \\
\hline 0.8 & -85.0 & 0.4 & 60.0 & 6.3 & 11.6 \\
\hline
\end{tabular}

Using the calculated results in Table 3-6 for $\boldsymbol{I}_{h 0}$ and $\boldsymbol{I}_{h c}$ as input (current sources) into an ATP model of Figure 3-10, a comparison between the calculated and simulated results for $\boldsymbol{I}_{h}$ are presented in Table 3-7. The rms values compare favourably but once again slight differences in the phase angles are seen, as highlighted in section 3.2, The differences may be due to manner in which ATP does the simulation or the MS Excel calculation and the number of decimal points used (although all input values were kept the same). The calculated results are deemed to be verified. 
Table 3-7. ATP simulated results

\begin{tabular}{|c|c|c|c|}
\hline \multicolumn{2}{|c|}{ I $h$ calculated } & \multicolumn{2}{|c|}{$\boldsymbol{I}_{h}$ simulated } \\
\hline $\operatorname{Mag}[A]$ & Angle $\left[{ }^{\circ}\right]$ & $\operatorname{Mag}[A]$ & Angle $\left[{ }^{\circ}\right]$ \\
\hline 0.3 & -83.3 & 0.3 & -82.4 \\
\hline 0.5 & -135.5 & 0.5 & -134.6 \\
\hline 0.8 & -153.6 & 0.8 & -152.7 \\
\hline 1.0 & -124.5 & 1.0 & -123.6 \\
\hline 1.2 & -128.0 & 1.2 & -127.1 \\
\hline 1.4 & -130.6 & 1.4 & -129.6 \\
\hline 0.5 & -73.4 & 0.5 & -72.5 \\
\hline 0.7 & -85.0 & 0.7 & -84.2 \\
\hline 0.8 & -92.1 & 0.8 & -91.2 \\
\hline
\end{tabular}

\section{Visualisation of HVM}

The goal of the harmonic vector method is to determine the magnitude of the harmonic sources (as part of a Norton equivalent circuit) that exist in the upstream network and in the load, depicted by $\boldsymbol{I}_{h 0}$ and $\boldsymbol{I}_{h c}$ in Figure 3-10. The amount of the harmonic current at both sources in Figure 3-10 is a factor of the harmonic current $\boldsymbol{I}_{h}$, the harmonic voltage $\boldsymbol{V}_{h}$ measured at the PoC and the respective harmonic impedances of the upstream network and the load.

Figure 3-11 is a repeat of Figure 2-11 but with the symbols as illustrated in Figure 3-10. Figure 3-11 shows an example phasor diagram of how the measured harmonic current $\boldsymbol{I}_{h}$ at the PoC is constituted through the contributions of $\boldsymbol{I}_{h c}$ and $\boldsymbol{I}_{h 0}$ according to [13].

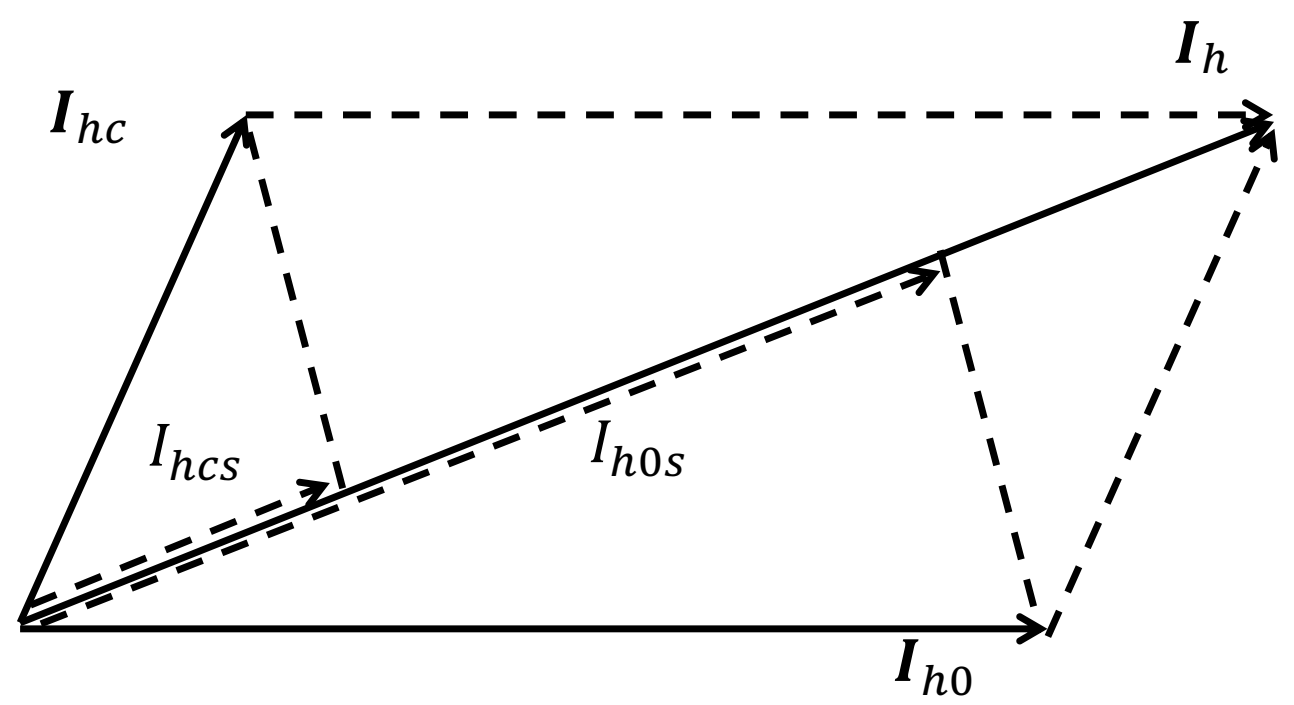

Figure 3-11. Example of utility and customer contributions to the measured current phasor at the PoC [13]

Where;

$\boldsymbol{I}_{h 0}$ : harmonic current phasor from utility network

$\boldsymbol{I}_{h c}$ : harmonic current phasor from load

$\boldsymbol{Z}_{h}$ : complex harmonic impedance of utility network

$\boldsymbol{Z}_{h c}$ : complex harmonic impedance of load

$\boldsymbol{I}_{h}$ : measured harmonic current phasor at the $\mathrm{PoC}$ 
$\boldsymbol{I}_{h c s}: \quad$ load's harmonic current contribution of $\boldsymbol{I}_{h c}$ to $\boldsymbol{I}_{h}$ (chart visualise the scalar value)

$\boldsymbol{I}_{h 0 s}$ : network's harmonic current contribution of $\boldsymbol{I}_{h 0}$ to $\boldsymbol{I}_{h}$ (chart visualise the scalar value)

A result from Table 3-6 is visualised to illustrate the harmonic current $\boldsymbol{I}_{h}$ and its constitution through $\boldsymbol{I}_{h 0}$ and $\boldsymbol{I}_{h c}$. Only one result will be presented to limit the number of charts. Figure 3-12 presents the harmonic phasors $\boldsymbol{I}_{h}, \boldsymbol{I}_{h 0}$ and $\boldsymbol{I}_{h c}$ when;

$\boldsymbol{I}_{h} \quad=1.4 \angle-130.6^{\circ} \mathrm{A}$ at the $5^{\text {th }}$ harmonic and,

$V_{h} \quad=236.44 \angle 36.6^{\circ} \mathrm{V}$ at the $5^{\text {th }}$ harmonic.

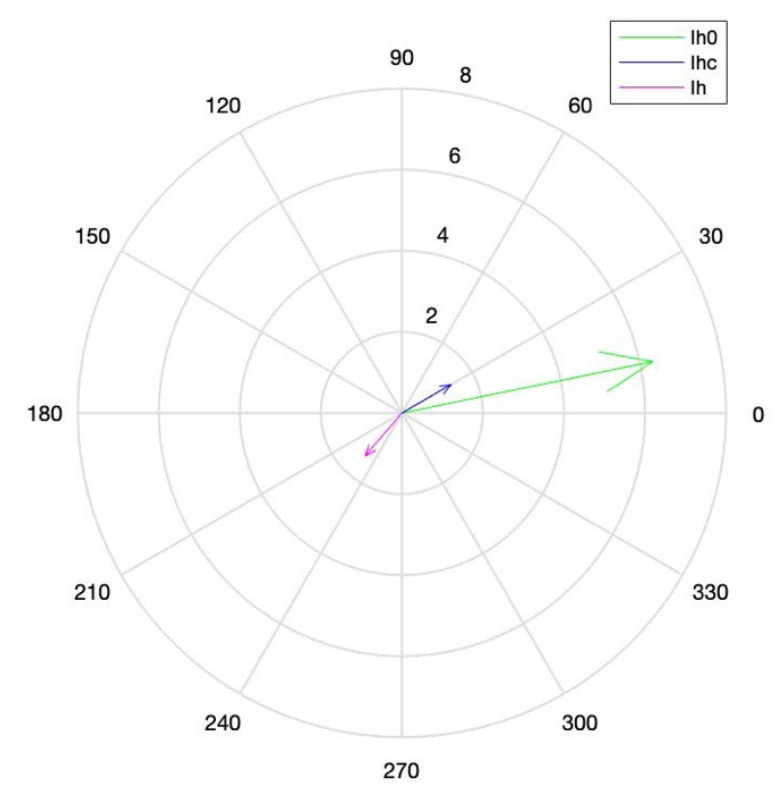

Figure 3-12. Visualisation of the network harmonic current and the load harmonic current for Norton equivalent circuits when $\mathbf{I}_{h}=1.4 \angle-130.6^{\circ}$ A using the harmonic vector method.

$\boldsymbol{I}_{h 0}$ and $\boldsymbol{I}_{h c}$ does not represent the harmonic emission from the respective harmonic sources, but rather the harmonic current phasors to be used in the Norton equivalent circuits as illustrated in Figure 3-10.

By application of the superposition method discussed in section 2.5.5, the network $\left(\boldsymbol{I}_{h 0_{s}}\right)$ and load's ( $\left.\boldsymbol{I}_{h c s}\right)$ harmonic contribution to $\boldsymbol{I}_{h}$ (harmonic emission) is determined using equations (10) and (11) from the results of equations (31) and (32).

Table 3-8 list the results of $\boldsymbol{I}_{h{ }_{s}}$ and $\boldsymbol{I}_{h c s}$ from equations (10) and (11). 
Table 3-8. Results of superposition method

\begin{tabular}{|c|c|c|c|c|c|}
\hline \multicolumn{2}{|c|}{$\boldsymbol{I}_{h}$} & \multicolumn{2}{c|}{$\boldsymbol{I}_{\text {hcs }}$} & \multicolumn{2}{c|}{$\boldsymbol{I}_{h 0_{s}}$} \\
\hline Mag [A] & Angle [\%] & Mag [A] & Angle [ $\left.{ }^{\circ}\right]$ & Mag [A] & Angle [ $\left.{ }^{\circ}\right]$ \\
\hline 0.3 & -83.3 & 0.2 & 3.3 & 0.4 & -53.3 \\
\hline 0.5 & -135.5 & 0.6 & 3.3 & 0.4 & -53.3 \\
\hline 0.8 & -153.6 & 1.0 & 3.3 & 0.4 & -53.3 \\
\hline 1 & -124.5 & 1.0 & 33.3 & 0.4 & -53.3 \\
\hline 1.2 & -128 & 1.2 & 33.3 & 0.4 & -53.3 \\
\hline 1.4 & -130.6 & 1.4 & 33.3 & 0.4 & -53.3 \\
\hline 0.5 & -73.4 & 0.2 & 63.3 & 0.4 & -53.3 \\
\hline 0.7 & -85 & 0.4 & 63.3 & 0.4 & -53.3 \\
\hline 0.8 & -92.1 & 0.6 & 63.3 & 0.4 & -53.3 \\
\hline
\end{tabular}

Similar to Figure 3-12, the harmonic phasors are visualised in Figure 3-13 for the same conditions as presented above for $\boldsymbol{I}_{h}$ and $\boldsymbol{V}_{h}$.

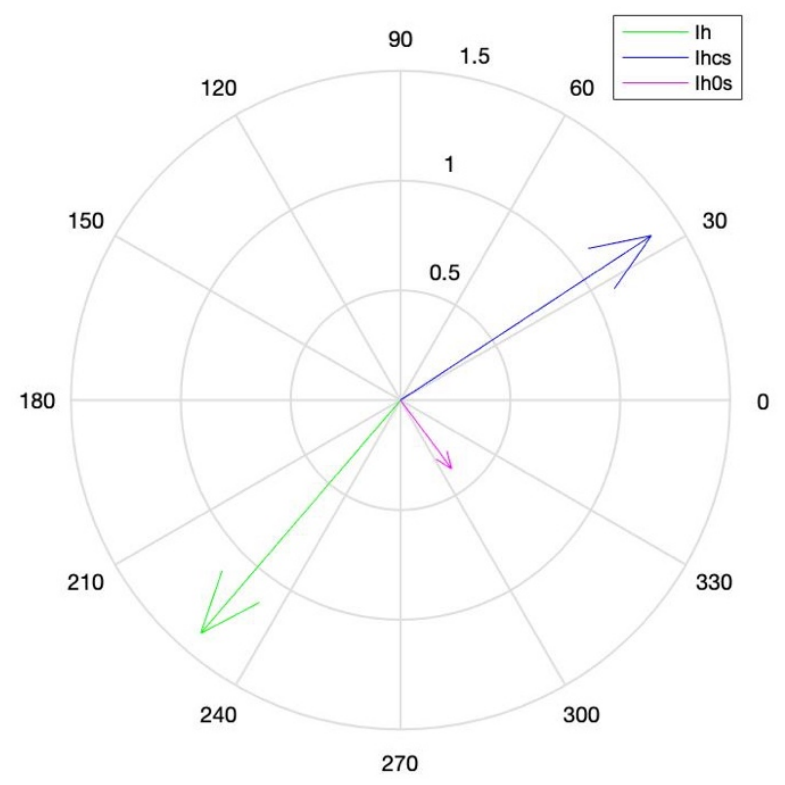

Figure 3-13. Illustration of superposition method of the harmonic contributions from the load and the network to a single measurement point.

Summation of $\boldsymbol{I}_{h c s}$ and $\boldsymbol{I}_{h 0_{s}}$ results in the harmonic current $\boldsymbol{I}_{h}$ as confirmed by (12). $\boldsymbol{I}_{h c s}$ and $\boldsymbol{I}_{h 0_{s}}$ is the harmonic emission components of the non-linear load and the upstream network, respectively.

\section{Discussion}

Single-point harmonic voltage and current phasor measurements at a POC and an accurate harmonic impedance profiles for both the network and load side are key to the application of the harmonic vector method. Using the HVM alone will results in parameters to be used in Norton equivalent circuits of the upstream network and the non-linear load. In order to evaluate the harmonic emission values from each source (upstream network and non-linear load), superposition must be applied to identify the harmonic emission from the non-linear load only or the upstream network only. 
Under the controlled conditions possible through the simulations done in this section, the harmonic vector method was applied to determine harmonic emissions from the load and from the upstream network to the measured harmonic current at the PoC.

For a pragmatic engineer, this method may present a challenge if all of the information is not at hand such as the harmonic impedance of the non-linear load, which is often not available. Measurement of harmonic phasors is a further constraint of this method as PQ recorders that comply to IEC 61000-4-30 only result in rms values rendering this approach to harmonic emission assessment useless.

\subsection{Harmonic emission assessment by the range of secondary cancellation between harmonic phasors}

The range of secondary cancellation (RSC) results in a maximum and minimum phase angles in between which current harmonic phasors will either have a positive or negative impact on the harmonic voltage distortion at the PoC. The concept is based on following variables, which will result in various RSC ranges for various harmonic orders as networks are not fixed and harmonic impedances vary;

- the harmonic impedance phasor of the upstream network,

- the background harmonic voltage phasor,

- the harmonic current phasor when the non-linear load is active.

The objective of this section is to simulate the results for the range of secondary cancellation under controlled conditions and to evaluate the results against the theory presented in section 2.5.8. Results from the range of secondary cancellation will be obtained by applying (23) to the circuit diagram depicted in Figure 2-14 and using the parameters listed in section 3.2. Only the $5^{\text {th }}$ harmonic will be investigated as the goal is to investigate the range of secondary cancellation theory.

Aggregated harmonic phasors ${ }^{7}$ being regarded as prevailing [73] are called for in (23). In order to improve the prevailing phasor that represent the simulation dataset, an increased number of sample data points will be used for the range of secondary cancelation simulation only. Table A13 (in Appendix A) is an extension of the input data used in Table 3-1.

Two conditions will be simulated;

- when the harmonic source exist in the load,

- when the harmonic source exist in the network.

Table 3-9 lists the maximum and minimum phase angle results of the RSC calculation from (23), by using the input values as stipulated above. The presented results in Table 3-9 list the condition when the harmonic source exist in the load.

\footnotetext{
${ }^{7}$ The aggregation of harmonic phasors is discussed in detail in chapter 5
} 
Table 3-9 RSC $5^{\text {th }}$ harmonic values: harmonic source in the load.

\begin{tabular}{|c|c|c|}
\hline & \multicolumn{2}{|c|}{$\begin{array}{c}5^{\text {th }} \text { harmonic } \\
\text { I }_{h} \text { Level of prevalence }=0.87 \\
\end{array}$} \\
\hline & Mag & angle $\left[{ }^{\circ}\right]$ \\
\hline $\begin{array}{l}\text { Background } 5^{\text {th }} \text { harmonic voltage } \\
\text { distortion }[\mathrm{V}]\end{array}$ & 200 & 30.00 \\
\hline $5^{\text {th }}$ harmonic prevailing current $[\mathrm{A}]$ & 1.3 & -110.4 \\
\hline $\begin{array}{c}5^{\text {th }} \text { harmonic network impedance } \\
(\Omega)\end{array}$ & 31.6 & 18.4 \\
\hline Max range of cancelation $\left[^{\circ}\right]$ & & 95.6 \\
\hline Min range of cancelation $\left[^{\circ}\right]$ & & -72.4 \\
\hline
\end{tabular}

RSC minimum and maximum phase angles listed in Table 3-9 is visualised in range is visualised in Figure 3-14 in conjunction with the input harmonic current values listed in Table A13. The range of secondary cancellation is represented by two areas or ranges in Figure 3-14, a red area where the harmonic current phasor will have a negative impact on the harmonic voltage (increase the harmonic voltage) and a green area where the harmonic current will have a positive impact on the harmonic voltage (decrease the harmonic voltage).

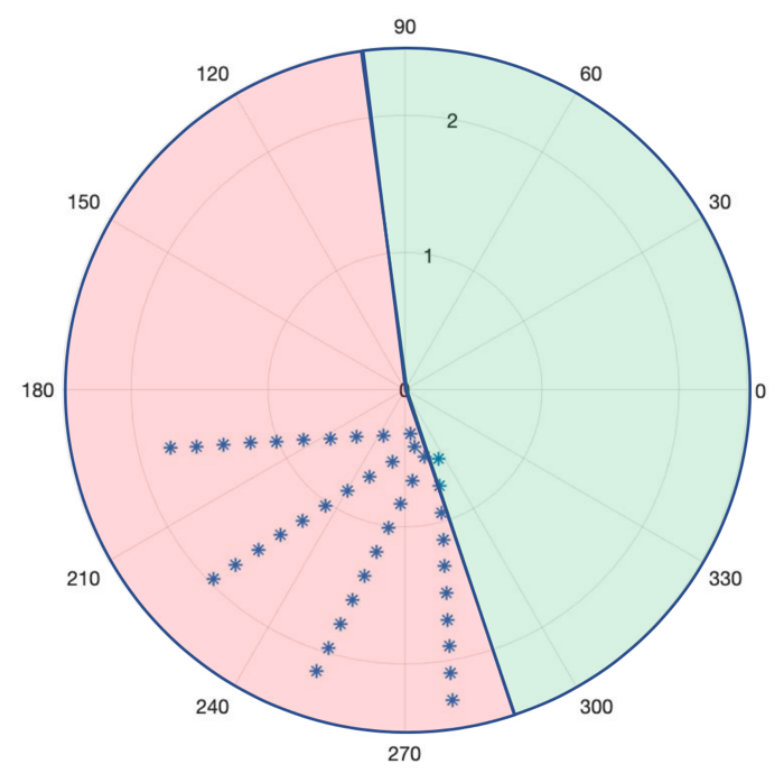

Figure 3-14. Range of secondary cancellation with harmonic current values: Harmonic source exists in load

Figure 3-14 shows that the input values used in the simulation, shown by blue stars, operate in the red area which will have a negative impact on the harmonic voltage at the PoC as expected.

Table 3-10 lists the maximum and minimum phase angles for the case when the harmonic source in the upstream supply network. Harmonic voltage $\boldsymbol{E}_{h 0}$ is varied as listed in Table A14 to simulate the harmonic source in the network. Harmonic current $\boldsymbol{I}_{h}$ is obtained by application of (28) with $\boldsymbol{I}_{h c}$ equal to $0.00001 \angle 0^{\circ} \mathrm{A}$. 
Table 3-10 RSC calculated values for the $5^{\text {th }}$ harmonic when harmonic source is in the upstream network.

\begin{tabular}{|c|c|c|}
\hline \multirow{2}{*}{} & \multicolumn{2}{|c|}{$\begin{array}{c}\boldsymbol{5}^{\text {th }} \text { harmonic } \\
\text { Ih } \text { Level of prevalence }=\mathbf{0 . 8 4}\end{array}$} \\
\cline { 2 - 3 } Mag & angle [ $^{\circ}$ ] \\
\hline $\begin{array}{c}\text { Background } 5^{\text {th }} \text { harmonic voltage } \\
\text { distortion }[\mathrm{V}]\end{array}$ & 121.1 & 48.3 \\
\hline $\begin{array}{c}5^{\text {th }} \text { harmonic prevailing current } \\
{[\mathrm{A}]}\end{array}$ & 0.2 & -38.3 \\
\hline $\begin{array}{c}5^{\text {th }} \text { harmonic network impedance } \\
(\Omega)\end{array}$ & 31.6 & 18.4 \\
\hline $\begin{array}{c}\text { Max range of cancelation }\left[{ }^{\circ}\right] \\
\text { Min range of cancelation }\left[{ }^{\circ}\right]\end{array}$ & & 118.0 \\
\hline
\end{tabular}

Figure 3-15 visualise the range of secondary cancellation minimum and maximum values listed in Table 3-10 in conjunction with the calculated harmonic current values $\boldsymbol{I}_{h}$.

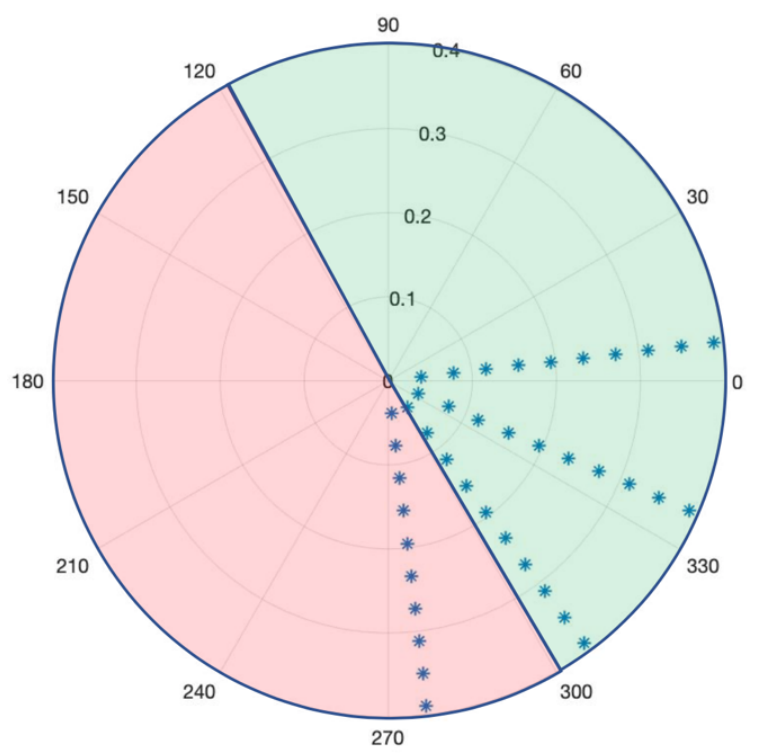

Figure 3-15. Range of secondary cancellation with harmonic current values: Harmonic source exists in network

Majority of the harmonic current data points $\boldsymbol{I}_{h}$ presented in Figure 3-15 operate in the green area indicating that these harmonic currents do not have a negative impact on the harmonic voltage of the network, as expected. There are however a few harmonic current data points that operate in the negative area which indicate that the non-linear load has a negative impact, this is not expected as the harmonic source in the load is negligible.

It is suspected that the wide angular range of the harmonic voltage, $\boldsymbol{E}_{h 0}$, input values which range from $0^{\circ}$ to $90^{\circ}$ might be the cause of some of the harmonic current $\boldsymbol{I}_{h}$ operating in the red area in Figure 3-15. In a real network such a high angular swing is not expected in the upstream network harmonic source, unless there are contingencies or reactive compensation equipment switching in the network that impact the harmonic impedance of the network.

To test the above mentioned suspicion the RSC calculations, for the case when the harmonic source is in the network, is redone with the angular range of the harmonic voltage $\boldsymbol{E}_{h 0}$ reduced to between $30^{\circ}$ and $45^{\circ}$ as listed in Table A15 in Appendix A. The revised input values in Table A15 is applied to (28) to result $\boldsymbol{I}_{h}$. Results for the RSC calculations are listed in Table 3-11. 
Table 3-11 RSC calculated values for the $5^{\text {th }}$ harmonic when harmonic source is in the upstream network (reduced angular range for input voltage harmonic).

\begin{tabular}{|c|c|c|}
\hline & I Leve & onic \\
\hline & Mag & angle $\left[{ }^{\circ}\right]$ \\
\hline $\begin{array}{l}\text { Background } 5^{\text {th }} \text { harmonic voltage } \\
\text { distortion [V] }\end{array}$ & 36.5 & 38 \\
\hline $5^{\text {th }}$ harmonic prevailing current $[\mathrm{A}]$ & 0.07 & -48.6 \\
\hline $\begin{array}{c}5^{\text {th }} \text { harmonic network impedance } \\
(\Omega)\end{array}$ & 31.6 & 18.4 \\
\hline Max range of cancelation $\left[{ }^{\circ}\right]$ & & 107.7 \\
\hline Min range of cancelation $\left[^{\circ}\right]$ & & -68.7 \\
\hline
\end{tabular}

Table 3-11 list the minimum and maximum angles for the range of secondary cancellation with the reduced angular range as discussed above. Observe that all of the data points for $\boldsymbol{I}_{h}$ operate in the green area indicating that the non-linear load has a positive effect on the harmonic voltage at the PoC.

A slight change in the minimum and maximum angles can also be observed due to the reduction in the angular range of the input harmonic voltage. This is due to the reduction of the prevailing harmonic phase angle used for this case study.

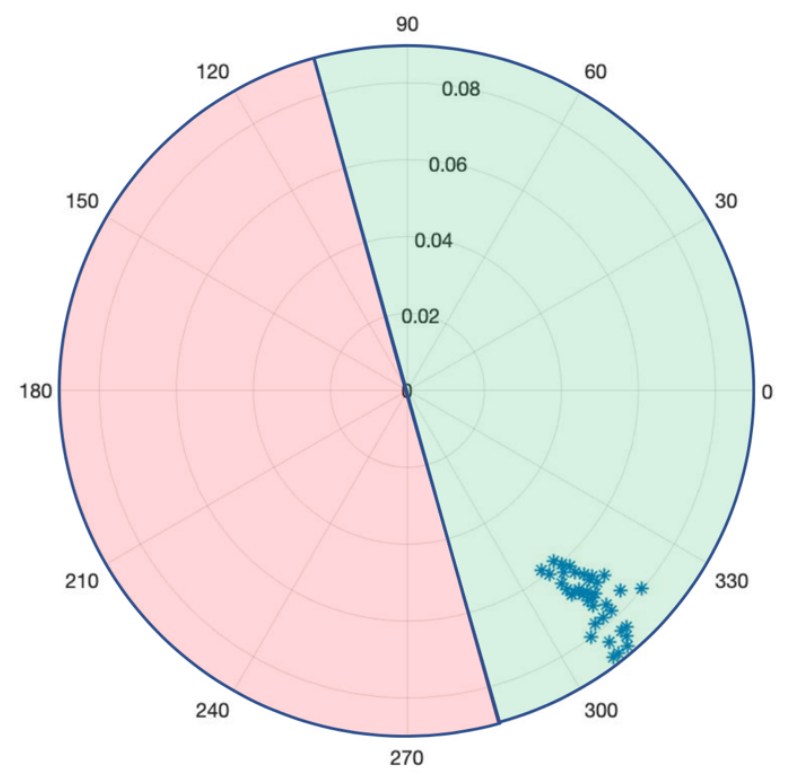

Figure 3-16. Range of secondary cancellation with harmonic current values: Harmonic source exists in network with reduced angular range of harmonic voltage

\section{Considerations for the range of secondary cancellation}

The range of secondary cancellation is a relatively new approach to evaluate the impact of harmonic currents originating from a non-linear load onto the harmonic voltage at a $\mathrm{PoC}$ of a supply network.

Through simulation of a simple circuit, the range of secondary cancellation was confirmed under controlled conditions where the harmonic source was placed in the upstream network and the load side. 
Application of this approach requires the measurement of harmonic voltage and harmonic current phasors at a single measurement point. Knowledge of the complex harmonic impedance of the network is the last parameter required to calculate the minimum and maximum angles for the range of secondary cancellation.

Harmonic phasor data will result in a large volume of data, but aggregation of harmonic phasors is a new concept that will reduce the volume of data to be considered. This concept is discussed in detail in Chapter 5.

\subsection{Conclusion}

For the controlled conditions presented (single non-linear load), where either the harmonic current or the harmonic voltage was changed depending on the simulation undertaken, the application of the harmonic emission assessment methods were achieved.

Circuit analysis was firstly confirmed by comparison to an ATP model of the circuit under investigation. The same circuit was used throughout this chapter except for two cases;

- Where multiple non-linear loads were simulated and,

- Where the harmonic emission assessment called for Norton equivalent circuits to be used.

Simulation of the various methods highlighted the usefulness of some methods and the challenges that a pragmatic engineer may face in applying some of the harmonic emission assessment methods.

The CIGRE C4.109 scatterplot method called for harmonic voltage and harmonic current rms value to be measured which is easily archived with modern day power quality recorders. Limitation in the CIGRE method is when measurements are taken at a single measurement point as discrimination of harmonic emission values is not possible in this qualitative method.

All harmonic emission methods studied in this chapter, except the CIGRE C4.109 scatterplot, make use of harmonic voltage and current phasors. Harmonic phasors may not be available in practical applications as harmonic phasors obtained by the 10/12 cycle block of data are aggregated to rms values. This important deficiency was overcome in this thesis by the aggregation of harmonic phasors, discussed later in this document. Most PQ instruments will not be able to record aggregated harmonic phasors, rendering all those harmonic emission methods that requires harmonic phasors, useless for real life application. 


\section{CHAPTER 4: Limitations for field application of harmonic emission assessment}

\subsection{Introduction ${ }^{8}$}

Instrumentation that can retain harmonic phasors are not readily available as the IEC 61000-4-30 PQ measurement principles will not retain phase angle during aggregation to reduce field data. As discussed earlier, harmonic phasors are derived from the 10/12 cycle block values but not automatically retained as most applications of PQ data focus on benchmarking the voltage quality against a national PQ standard [7] where 10-min rms values are sufficient.

Harmonic emission assessment is not possible when making use of only the rms values of harmonic phasors. This chapter use real-life field data to validate that the deficiency in accepted approaches to harmonic emission assessment is:

a. real,

b. that known methodologies will not withstand forensic scrutiny when disputes require litigation.

Only the $5^{\text {th }}$ and $7^{\text {th }}$ harmonic orders are considered in this chapter as the principles of the harmonic emission assessment does not change with harmonic order. It is also not important to consider all harmonic orders during this investigation as from a metrological point of view, the lower the harmonic order the higher the measurement certainty, supporting the scientific validity of results.

PQ recorders were deployed for the field measurements that are compliant to the latest edition 3 (during the time of investigation) Class A measurement requirements of the IEC 61000-4-30. Valid calibration certificates for these instruments were obtained to ensure that the measurement results are as good as practically possible from a real-world metrological point of view.

Only rms harmonic phasor values are required during the application of the CIGRE/CIRED C4.109 scatterplot method for harmonic emission assessment. This method is prescribed in the grid code agreement between the RPP (PV farm) pertaining to this field study, and the national utility [1].

Harmonic emission assessment should be fair and only account for the harmonic emission that a specific RPP emit onto the network resulting in an increase of harmonic voltages at a Point of Connection (PoC). Voltage waveform distortion at a $\mathrm{PoC}$ in an interconnected network hosting multiple and distributed sources of voltage waveform distortion, is the result of the interaction between all of the non-linear components in the power system (injecting harmonic currents).

The above principle is confirmed in this chapter by showing that harmonic emission assessment at a single measurement point is useless. Discrimination between how much of harmonic emission is contributed by the upstream network and how much by the downstream network (normally some user) is not possible within the constraint of rms measurements at a single point.

To improve the understanding of the interaction between the utility network and the user (PV plant) connected downstream of the PoC, synchronous measurements at the utility $132 \mathrm{kV}$ network and at the $22 \mathrm{kV} \mathrm{PoC}$ were taken. Using GPS synchronisation, a time-stamp uncertainty of better than a 100 ns were achieved [92].

${ }^{8}$ Two peer-reviewed conference papers as well as a journal article were published on the research contained in this chapter [20], [93], [110]. 
Figure 4-1 presents the system and measurement configuration.

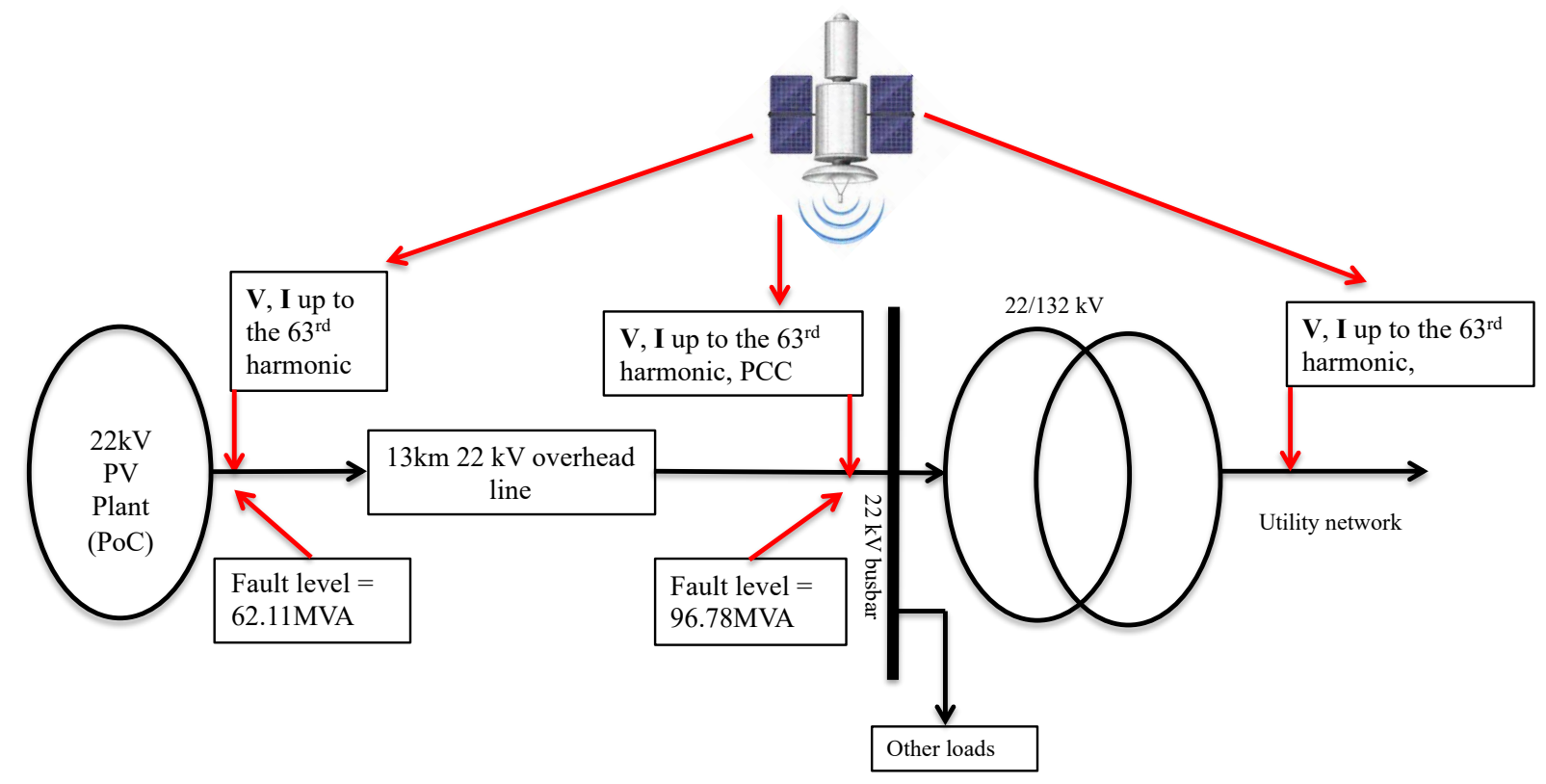

Figure 4-1 PV plant harmonic emission assessment, PoC defined at the $22 \mathrm{kV}$ connection downstream of the overhead line

\subsection{RMS harmonic data analysis when using single-point measurements}

Harmonic emission assessment is done in this section within the constraint of 10-min rms values of a single point of measurement. This reflects the typical dataset available to most field engineers as guided by the South African grid code for RPPs.

The goal of grid code compliance assessment is to benchmark harmonic distortion against the apportioned harmonic emission limits issued by the electrical utility. Having a single set of data, some interpretation is needed to understand if the user (PV plant in this case) complies to the harmonic emission limits.

\subsubsection{Analysis of $5^{\text {th }}$ and $7^{\text {th }}$ harmonic voltage and current data}

By comparing simultaneous datapoints of voltage and current harmonics, if voltage rise and current "follow" the first explanation would be that a voltage source in the upstream supply network "drive" the current into the PV plant. It could easily be interpreted the other way around, namely that when current rise and voltage "follow", the reason is that a current source "drive" the current through an impedance (i.e. the $22 \mathrm{kV}$ overhead line in Figure 4-1) and that the root-cause to the harmonic order under investigation, is indeed due to harmonic emission.

It is possible that both (upstream and downstream network) contribute. In other words when the upstream system "drive" current into the $\mathrm{PoC}$ and the downstream system "drive" current into the PoC? Who is to blame and for how much?

These questions are now carefully analysed by the type of field data readily available, such as when recorded by a PQ recorder located at the contractually agreed Point of Connection (PoC). This $22 \mathrm{kV}$ point at the output of the PV plant is shown in Figure 4-1 and the goal is to the assess the harmonic emission contributed by the 5 MVA PV plant alone [1], [93].

Figure 4-2 present a selection of 10-min $5^{\text {th }}$ harmonic voltage and current rms values for a single day when full production of PV energy was realised as no cloud cover existed and all PV panels 
were in service. The same is done for the $7^{\text {th }}$ harmonic voltage and current in Figure 4-3. First, consider the $5^{\text {th }}$ harmonic voltage and current shown in Figure 4-2.

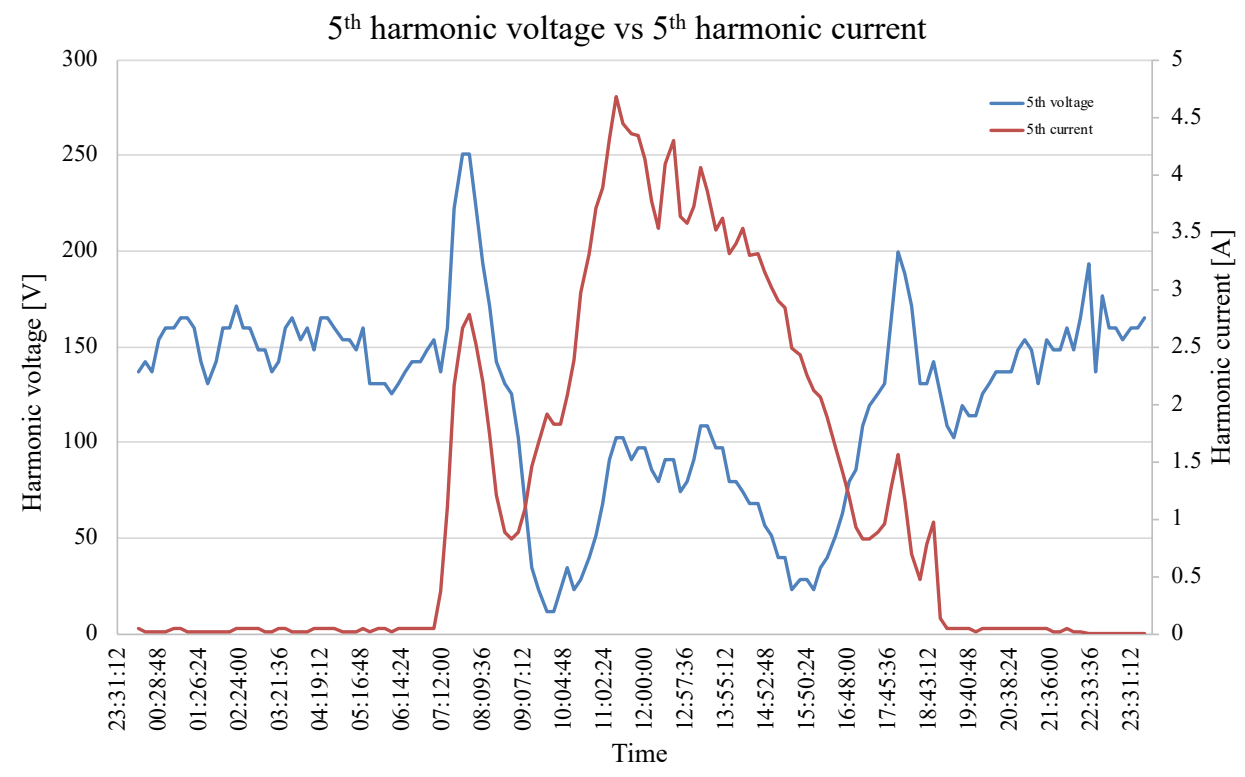

Figure 4-2. $5^{\text {th }}$ harmonic voltage vs $5^{\text {th }}$ harmonic current measurements at PV plant

It was discussed in Chapter 2 making use of Figure 2-14 that voltage harmonic emission $\left(\boldsymbol{E}_{h c}\right)$ realises when the harmonic voltage phasor at the non-linear load $\left(\boldsymbol{V}_{h}\right)$ is larger than the harmonic voltage phasor of the upstream network $\left(\boldsymbol{E}_{h 0}\right)[19]$ because harmonic current flows from the nonlinear load into the upstream network through a non-zero harmonic impedance $\left(\boldsymbol{Z}_{h}\right)$. Voltage harmonic emission $\left(\boldsymbol{E}_{h c}\right)$ is the result of the voltage drop across that impedance (resulting from the harmonic emission current represented by harmonic current phasor $\boldsymbol{I}_{h}$ ).

The above is perfectly valid if no other harmonic producing load located elsewhere in the network influence the harmonic voltage phasor $\left(\boldsymbol{V}_{h}\right)$ at the harmonic production load under consideration.

Using single-point measurements, it may be concluded that if the magnitude of the harmonic voltage phasor $\boldsymbol{E}_{h 0}$ (that is the harmonic voltage phasor in the upstream network) decrease when the magnitude of the harmonic current increase, that is because the non-linear load is absorbing harmonic current $\boldsymbol{I}_{h}$ (a voltage drop occurs over the upstream network impedance). If harmonic voltage $\boldsymbol{E}_{h 0}$ increases as a result of an increase in harmonic current $\boldsymbol{I}_{h}$, then the non-linear load is regarded as emitting harmonic current into the upstream network. Observe that the harmonic phase angles of the harmonic current phasors are not known ("direction" cannot be determined).

Figure 4-2 indicate an uncorrelated relation between the $5^{\text {th }}$ harmonic current and $5^{\text {th }}$ harmonic voltage. At some instances it seems that the PV plant emit harmonic emission (early morning and evening) as voltage increase when current increase. During night-time limited reduction of the harmonic voltage is noted and possibly that level of harmonic voltage can be regarded as the "background" harmonic voltage. The latter is invalidated during the day as there are periods where harmonic current increase and the harmonic voltage decrease. Was it the "background" harmonic voltages in the network which decreased or was it due to the PV plant absorbing harmonic current?

IEC 61000-3-6 [4] base the concept of harmonic emission on harmonic currents emitted by a harmonic producing load (flowing from the distorting load into the utility network). This cannot be determined from Figure 4-2 as at some points harmonic current seems to be emission and some seems to be "absorbed". The latter scenario (absorbing harmonic currents) is of benefit to the 
electrical utility and in treating the PV plant in a fair manner, the PV plant should be acknowledged for this "help" to reduce harmonic currents.

This demonstrate the dilemma at hand to the field engineer who has to apply a standardised methodology in assessing harmonic emission. A fair methodology should only use that harmonic currents that are "harmonic emission" as defined by the IEC 61000-3-6 principle.

It is therefore possible when assessment is based on the whole dataset of $5^{\text {th }}$ harmonic current rms values (i.e. measured over a week period), that some values can exceed the apportioned harmonic limits. Knowledge is needed to determine which values in the dataset are not "emission" but "absorbed" harmonic currents. This knowledge is not available when considering only single-point rms values of harmonic current and voltage phasors.

RPPs should only account for the harmonic emission being injected into the utility network. The utility could argue that if the PV plant was not connected at all, no harmonic currents would flow, but connecting a PV plant to sell energy is fundamentally part and parcel of the Electricity Supply Industry (ESI) and some consideration to ethical and fair treatment is needed. A network connection is needed to sell energy and if the change in network impedance cause the flow of harmonic currents not deliberately caused by the PV plant (for example), it has to be taken into account during harmonic emission assessment.

The above dilemma is not encountered when Figure 4-3 is analysed. The $7^{\text {th }}$ harmonic current correlate well with the $7^{\text {th }}$ harmonic voltage for almost the entire energy production cycle. It seems fair to conclude that all $7^{\text {th }}$ harmonic current datapoints can be considered as harmonic emission.

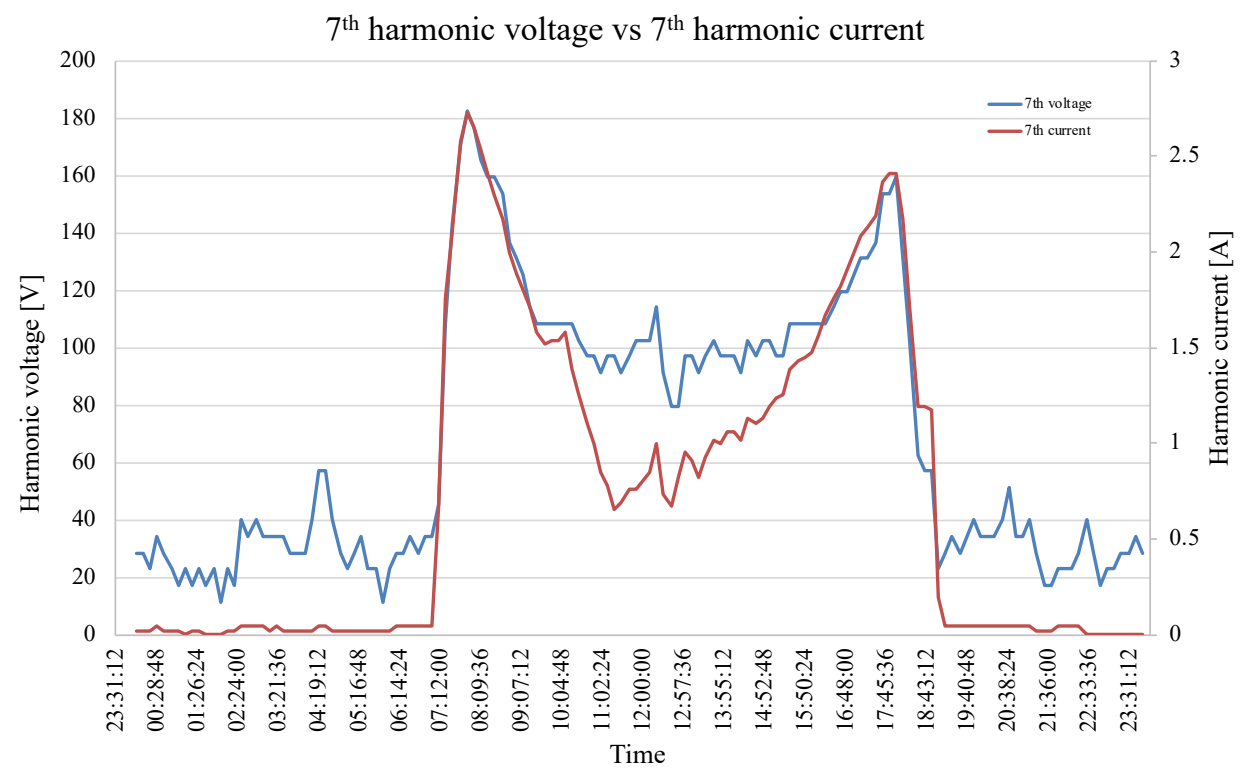

Figure 4-3. $7^{\text {th }}$ harmonic voltage vs $7^{\text {th }}$ harmonic current measurements at PV plant

From the discussion on the $5^{\text {th }}$ harmonic voltage and current, it seems that a methodology is needed to identify from the whole dataset those values to be regarded as harmonic emission. Take into account that at this point of Chapter 4, the assumption is that the dataset is obtained from a normal PQ recorder that retain only 10-min rms values and when this instrument is compliant to the Class A measurement requirement of IEC 61000-4-30 edition 3, it is concluded that the metrology is the best available to the average field engineer. Measurement uncertainty is considered to be as low as practically possible. 
During the aggregation of parameters calculated from the IEC 61000-4-30 10/12 cycle measurement block intervals, harmonic active power is also aggregated (to for example 10-min values). It is believed that harmonic active power has some information [63] on the "direction" of distortion as the sign of harmonic active power will be negative when the load under investigation, absorbs fundamental frequency active power (positive sign) but emits harmonic active power.

This principle can be applied to the total harmonic active power over all harmonic orders, or per harmonic order. Unfortunately, the latter only holds true when there is only one source of distortion (or harmonic currents). When more than one source exists, non-linear loads (or generators) can also exchange harmonic active power between them resulting in a nonlinear load with harmonic active power having the same positive sign as the fundamental frequency active power. This scenario can change a while later when the nonlinear interaction between the different nonlinear loads change. To fully understand this phenomenon requires synchronised measurements of harmonic active power at all nodes of the network under investigation [16], [78]. For most field engineers, that is not possible and why single-point measurements are mostly used.

It is possible to consider the whole dataset by evaluating the harmonic active power sign at each 10-min instance. When $P_{h}<0$ and $P_{l}>0$ is used as principle to categorise that specific 10-min harmonic current rms value as emission and as being absorbed when $P_{h}>0$ when $P_{1}>0$, a discriminative approach is now possible to scrutinise the whole dataset for "conditions of emission" in the attempt to be fair to the RPP. The direction of fundamental active power flow was defined to be from the power plant into the network, fundamental active power has a negative sign and has to be taken into account when the above explanation on the direction of harmonic active power is considered.

Harmonic active power discrimination was applied to the whole dataset that was shown in Figure 4-2 and Figure 4-3. The result is shown in Figure 4-4 and Figure 4-5 for the $5^{\text {th }}$ and $7^{\text {th }}$ harmonic voltage and current respectively.

During the night-time when the PV plant is not in generation, harmonic emission is not shown in Figure 4-4 and Figure 4-5. The same is done for both the $5^{\text {th }}$ and the $7^{\text {th }}$ harmonic around 9:30 and 11:00 (although for a short period) with the result that was is now shown, based on the sign of harmonic active power, is regarded as harmonic emission contributed by the PV plant.

Note that the $5^{\text {th }}$ harmonic voltage (Figure 4-4) increases when the $5^{\text {th }}$ harmonic current decreases from around 16:00 onwards. This phenomenon suggests that the $5^{\text {th }}$ harmonic current are being sunk by the PV plant. Although harmonic active power suggested that these datapoints are to be included during the harmonic emission assessment, from the latter observation it should not.

The above is an important validation of what is known in literature [56], [61], [94] namely that harmonic active power cannot be used to make any conclusion on harmonic emission. 


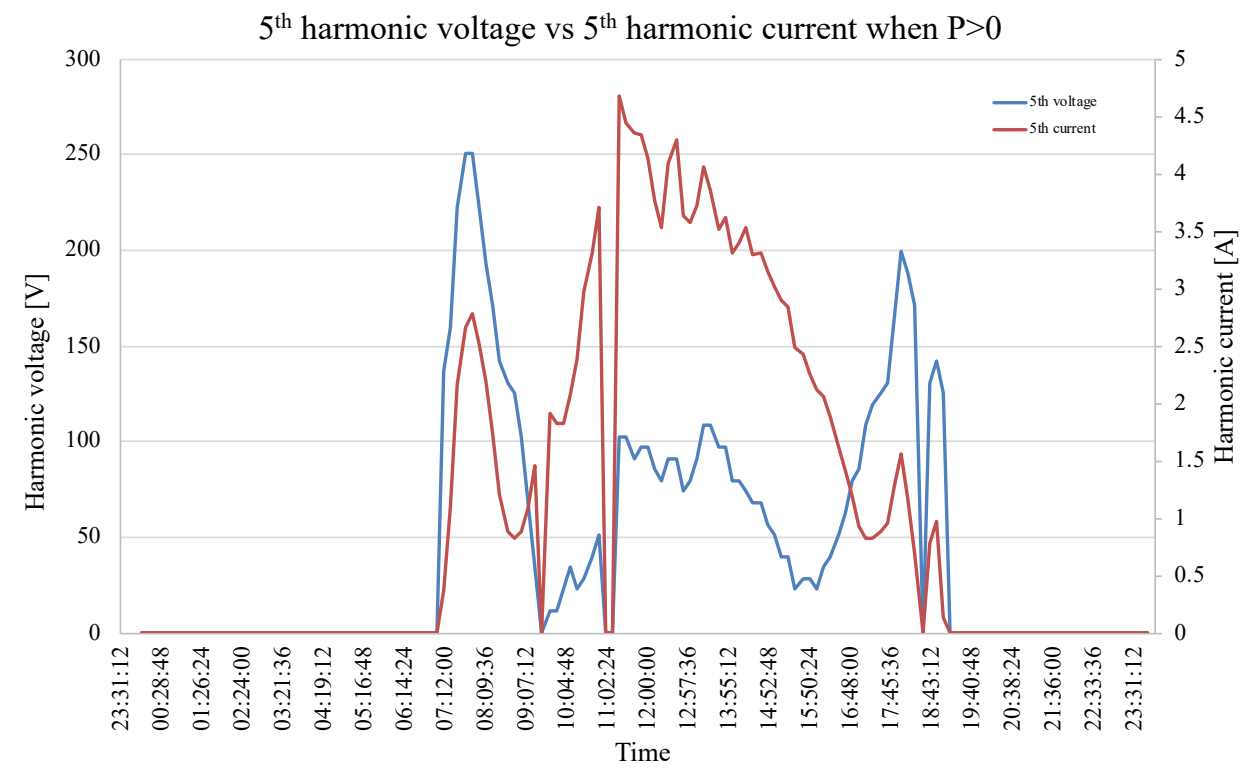

Figure 4-4. $5^{\text {th }}$ harmonic voltage vs $5^{\text {th }}$ harmonic current measurements at PV plant with only positive harmonic active power considered

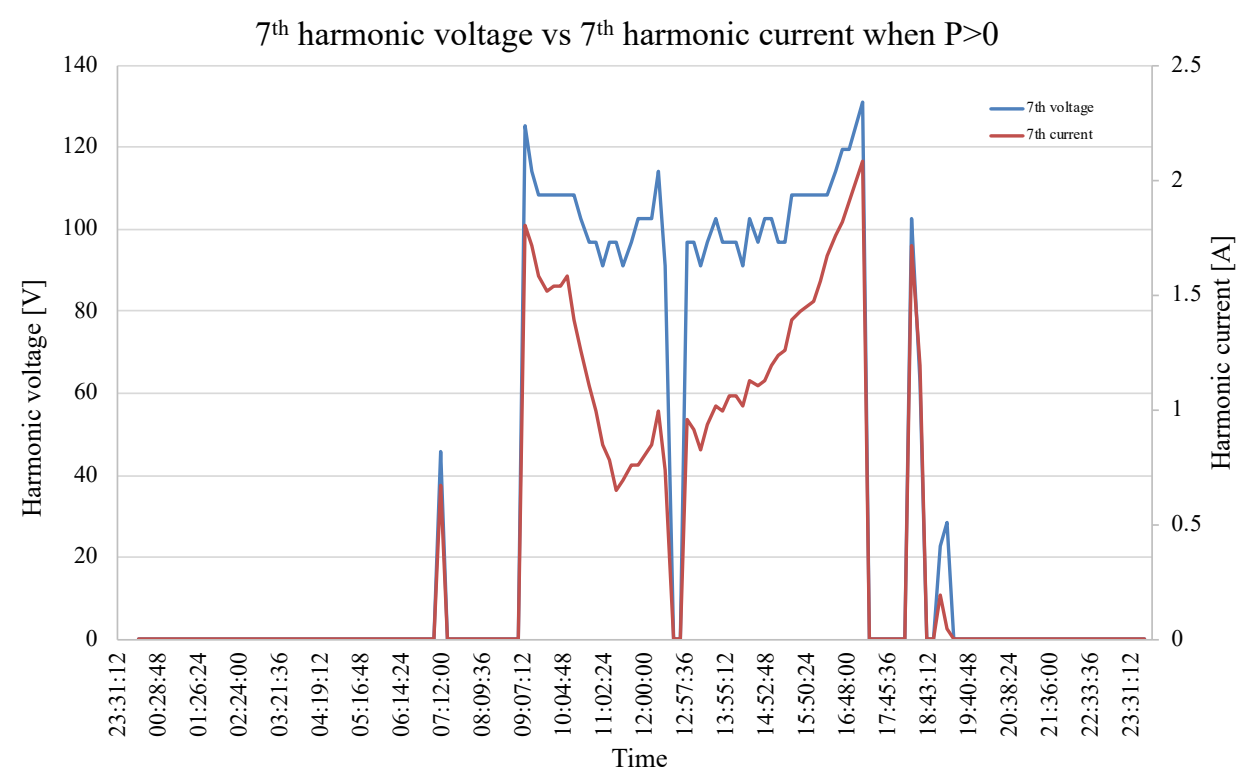

Figure 4-5. $7^{\text {th }}$ harmonic voltage vs $7^{\text {th }}$ harmonic current measurements at PV plant with only positive harmonic active power considered

It is concluded that using 10-min rms values of voltage and current harmonics, even when the dataset is being analysed using harmonic active power as discriminator, that no conclusion can be made on harmonic emission when more than one source of harmonic emission exists in an interconnected network. The latter principle was known from the referenced literature [56], [61], [94], but comprehensively analysed using field data and principles known to be used by field engineers.

\subsubsection{Application of CIGRE/CIRED C4.109 scatterplot method}

A limited number of harmonic emission assessment methodologies are listed in the South African grid code on renewable power plant integration [1], the aim of which is to treat all RPPs that 
integrate onto the South African network in a fair and equitable manner [95]. These prescribed methods were accepted by both industry (RPPs) and utilities taking into account the measurement constraints and existing PQ standards within South Africa. The CIGRE/CIRED C4.109 method of harmonic emission assessment, is referenced in SA grid code document [1] as a solution to better understand the contribution from the upstream (mostly utility) network and the downstream (mostly user) network. Using a dataset representing two full weeks measured at the PoC of the PV plant shown in Figure 4-1, the CIGRE/CIRED C4.109 scatterplot method is now applied.

A challenge is to obtain the harmonic impedance profile of the network and of the load (i.e. PV plant). Harmonic impedance of the upstream network may be obtained from the electrical utility (mostly estimated from a network equivalent model) but the load impedance is largely unknown. It is complex to accurately estimate or model the load impedance due to not only the non-linear principle of operation, many contingencies have to be taken into account such as part of the plant under maintenance (i.e. washing PV panels).

It is suggested in [71] that the harmonic impedance of the network is sufficient to derive the harmonic emission of the distorting load (discussed in section 2.5.7.)

During the field study pertaining to this chapter (configuration described in Figure 4-1), a "best" possible scenario existed to test the CIGRE/CIRED/C4.109 scatterplot method. The harmonic impedance profiles from the electrical utility could be estimated as shown in Figure 4-6.

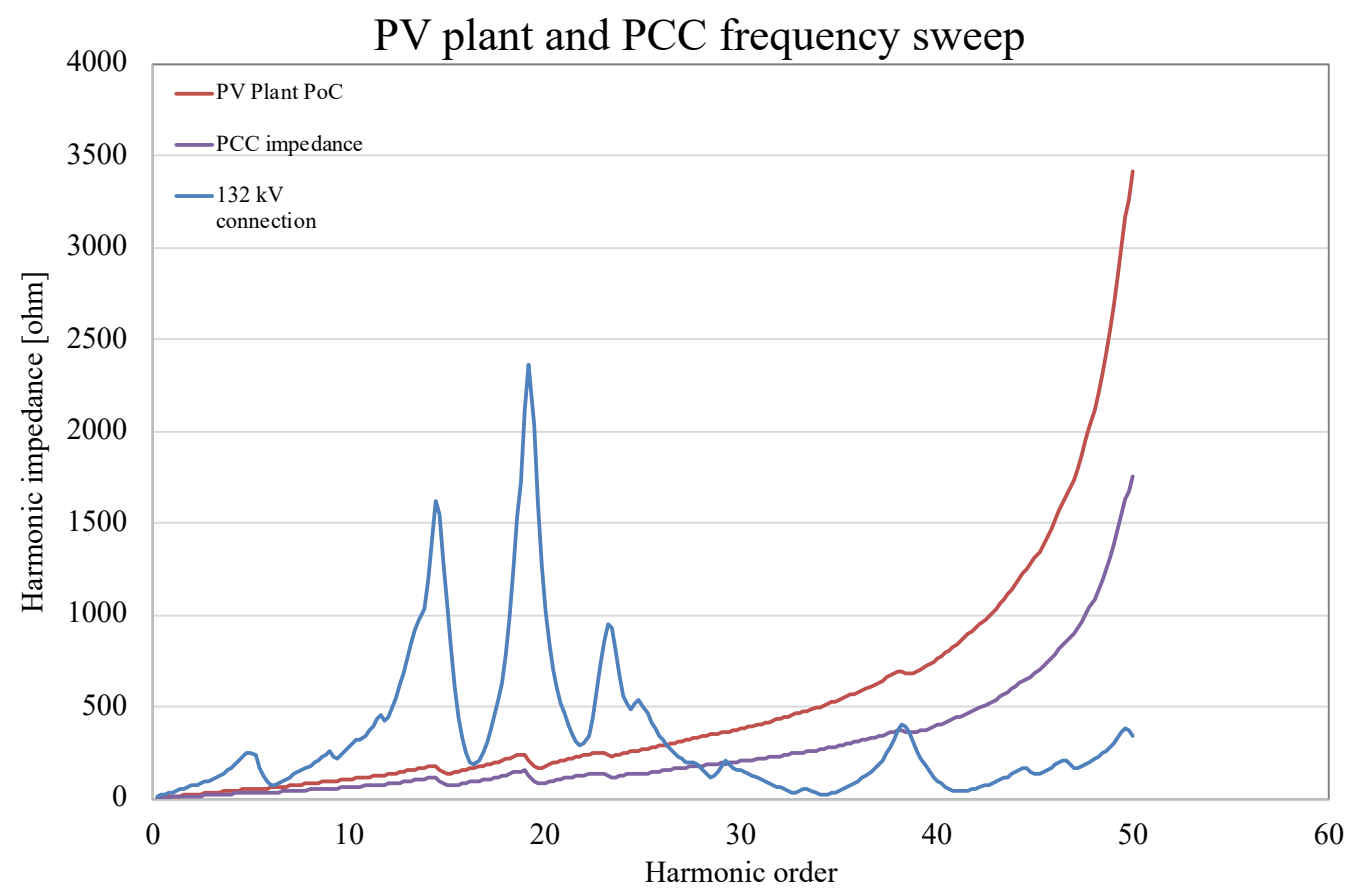

Figure 4-6. Frequency sweeps for the network under grid code compliance

Again, the focus is on harmonic emission at the $5^{\text {th }}$ and $7^{\text {th }}$ harmonic order. Application of the CIGRE/CIRED C4.109 scatter plot approach resulted in Figure 4-7 for the $5^{\text {th }}$ harmonic and in Figure 4-8 for the $7^{\text {th }}$ harmonic. The harmonic impedance profile denoted by $Z_{5}$ in Figure $4-7$ and $Z_{7}$ in Figure 4-8 indicate the impedance at the $22 \mathrm{kV} \mathrm{PoC}$ for the $5^{\text {th }}$ and $7^{\text {th }}$ harmonic respectively.

Two distinct groupings of the data points (green ellipses) are seen in Figure 4-7 spaced above and below the $Z_{5}$ impedance line. This confirms what was noted in section (4.2.1) namely that both the PV plant and the utility network contribute to harmonic emission at the $5^{\text {th }}$ harmonic order. Again, the dilemma is clear, how can harmonic emission from the PV plant be benchmarked against the contracted limits using only 10 -min rms values of harmonic voltages and currents? 
A definite alignment of the scatter points and the impedance loci is needed as discussed in section 2.5.7 to assign harmonic contribution to the upstream or downstream network at the PoC under investigation.

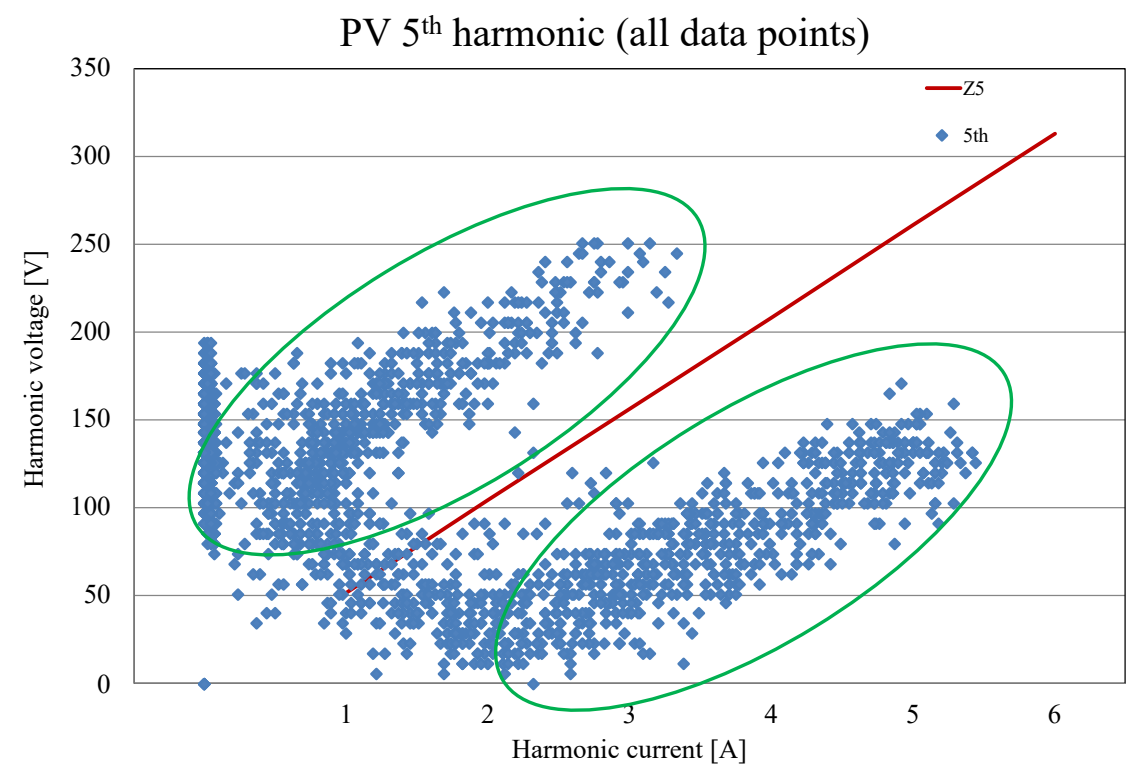

Figure 4-7. $5^{\text {th }}$ harmonic scatter plot at PV plant

The scatter plot shown in Figure 4-8 achieves what was intended by the CIGRE/CIRED C4.109 method, namely a relative close spacing around the impedance profile of the $7^{\text {th }}$ harmonic indicate that the PV plant (downstream network) is the source of the emission at $7^{\text {th }}$ harmonic order. These points remained somewhat scattered around the impedance line but compared to Figure 4-7, a distinct contribution of both the upstream and downstream network cannot be seen.

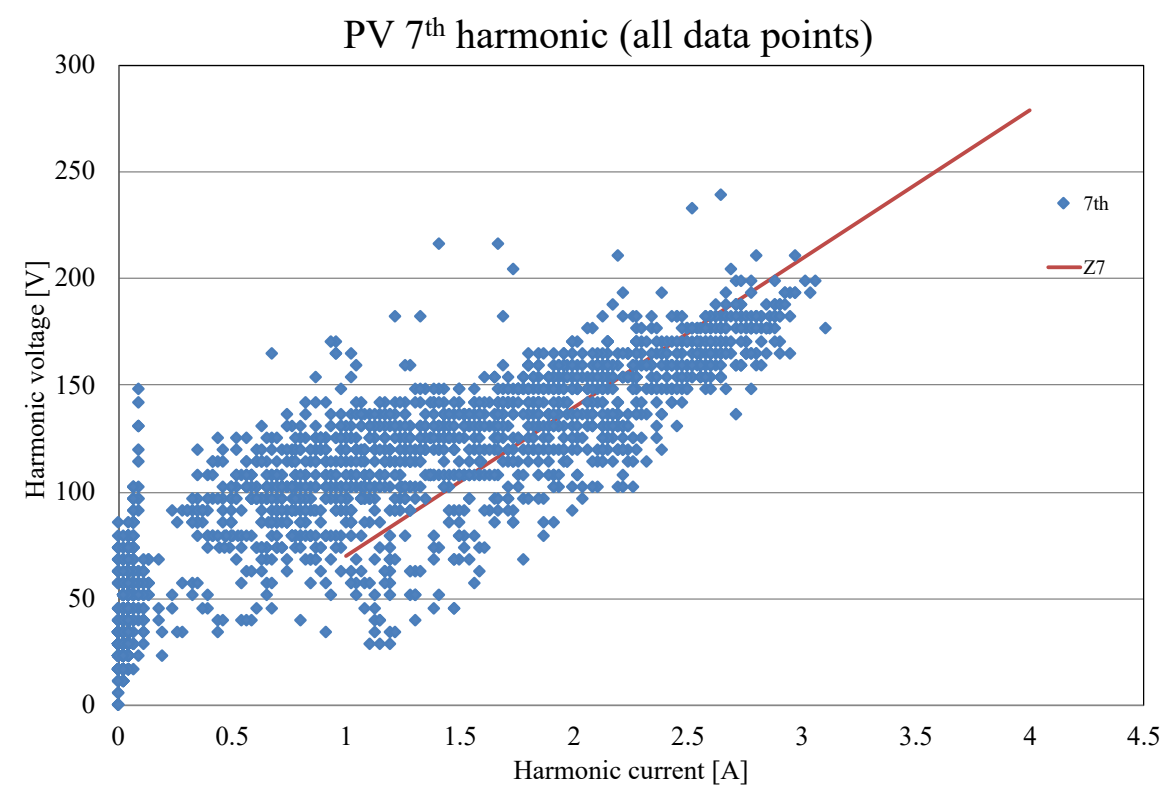

Figure 4-8. $7^{\text {th }}$ harmonic scatter plot at PV plant

In the next section 4.2 .3 , the $95^{\text {th }}$ percentile of the measured 10 -min rms values for harmonic current is used in deriving harmonic emission values as described the CIGRE/CIRED method [19]. 


\subsubsection{Harmonic emission calculation based on CIGRE/CIRED C4.109}

The emission of the PV plant alone cannot be determined with 10-min rms values as the measurement data set contains values which are due to both the PV plant and the network as shown. A fundamental principle on emission is applied by the CIGRE/CIRED C4.109 method [19] in defining emission as the condition when harmonic voltage of the PV plant is greater than the harmonic voltage of the network $\left|\boldsymbol{V}_{h}\right|>\left|\boldsymbol{E}_{h 0}\right|$. This is not possible with single-point measurements. Also, these single-point measurements have to be done synchronously as the measurements with the exact same time-stamping has to be tested for $\left|\boldsymbol{V}_{h}\right|>\left|\boldsymbol{E}_{h 0}\right|$.

Without availability to the above test, as possibly faced by most field engineers when doing a harmonic assessment, the assumption has to be made that all of measured harmonic values emanate from the PV plant (i.e. $\left|\boldsymbol{V}_{h}\right|>\left|\boldsymbol{E}_{h 0}\right|$ ). Harmonic current emission assessment is then based on the $95^{\text {th }}$ percentile of the measured harmonic current rms values [19].

As example in this section, the CIGRE/CIRED C4.109 method is applied to the $5^{\text {th }}, 7^{\text {th }}, 11^{\text {th }}, 13^{\text {th }}$, $23^{\text {rd }}$, and $25^{\text {th }}$ harmonic orders. Voltage harmonic emission follows from (19) with results tabulated in Table 4-1, contracted harmonic emission limits are listed in Table 4-2.

Table 4-1. PV Plant A harmonic emission values

\begin{tabular}{|c|c|c|c|c|c|c|}
\hline Harmonic no & $\mathbf{5}^{\text {th }}$ & $\boldsymbol{7}^{\text {th }}$ & $\mathbf{1 1}^{\text {th }}$ & $\mathbf{1 3}^{\text {th }}$ & $\mathbf{2 3}^{\text {rd }}$ & $\mathbf{2 5}^{\text {th }}$ \\
\hline $\begin{array}{c}95^{\text {th }} \text { percentile } \\
\text { harmonic current }[\mathrm{A}]\end{array}$ & 4.5 & 2.9 & 2 & 0.6 & 0.3 & 0.0 \\
\hline $\begin{array}{c}\text { Harmonic impedance } \\
(\Omega)\end{array}$ & 52.1 & 69.9 & 118.3 & 152.4 & 248.0 & 264.3 \\
\hline $\begin{array}{c}\text { Voltage harmonic } \\
\text { emission }(\mathrm{V})\end{array}$ & 233.1 & 202 & 230.6 & 86.9 & 69.5 & 0.0 \\
\hline
\end{tabular}

Table 4-2. PV Plant A contracted harmonic emission limits

\begin{tabular}{|c|c|c|c|c|c|c|}
\hline Harmonic no & $\mathbf{5}^{\text {th }}$ & $\boldsymbol{7}^{\text {th }}$ & $\mathbf{1 1}^{\text {th }}$ & $\mathbf{1 3}^{\text {th }}$ & $\mathbf{2 3}^{\text {rd }}$ & $\mathbf{2 5}^{\text {th }}$ \\
\hline $\begin{array}{c}\text { Contracted harmonic } \\
\text { current limits[A] }\end{array}$ & 4.1 & 2.3 & 1.4 & 1.3 & 0.3 & 0.2 \\
\hline $\begin{array}{c}\text { Contracted voltage } \\
\text { harmonic emission } \\
\text { limits (V) }\end{array}$ & 355.6 & 279.4 & 266.7 & 215.9 & 101.6 & 88.9 \\
\hline
\end{tabular}

Comparison of $95^{\text {th }}$ percentile harmonic current (deemed as emission) listed in Table 4-1 to the harmonic current limits in Table 4-2 indicates that the PV plant is exceeding the contracted limits for the $5^{\text {th }}, 7^{\text {th }}$ and $11^{\text {th }}$ harmonic orders. Assessment of the voltage harmonic emission values are below the contracted limits for all harmonic orders, but in the South African grid code, the harmonic current emission values are considered to be the main criteria for grid code compliance. Based on the above assessment, where all of the harmonic current values are considered as emission, the PV plant owner must mitigate the harmonic orders that exceed the limits.

\subsubsection{Conclusion on harmonic emission assessment using single-point measurements}

The notion that a non-linear load such as a PV plant will continuously emit harmonic emission is a fallacy as the harmonic emission is not only dictated by the operation of the PV plant but also by the upstream network operating conditions. Through the rudimentary application of the harmonic active power method to a measurement dataset and the correlation of voltage and current harmonics, it was shown that there is doubt in the constant harmonic emission of a PV plant. 
Single-point measurements were shown in this section to be not useful in the assessment of harmonic emission. As example, a PV plant owner will be forced to spend money on compensation equipment as a result of a fundamentally invalid result when standardised methodologies and data obtained from mostly PQ instruments are used.

Results obtained in this section made use of the best possible data when using a PQ instrument that is compliant to the latest version of the IEC 61000-4-30, edition 3 and certified to be compliant to the Class A measurement requirements. The metrology of this instrument represents the best possible scenario to most field engineers faced with harmonic emission assessment during for example a grid code compliance study at a PV plant (as used as example in this section).

From the results obtained and the evaluation of the CIGRE/CIRED C4.109 method, it seems that when using the above class of PQ data, that the principles of harmonic emission, namely that $\left|\boldsymbol{V}_{h}\right|>\left|\boldsymbol{E}_{h 0}\right|$ as explained in section 2.5 .7 could be improved using a set of measurements synchronised at the PoC of the load under investigation (PV plant in this case) and at the PCC somewhere upstream in the utility network. That is assuming the configuration of the network allows for such measurements as an impedance is needed between the PoC and the PCC as shown in Figure 4-1.

Synchronised measurements using rms PQ data is evaluated in the next section to understand if harmonic emission assessment can be improved.

\subsection{Evaluation of synchronised measurements across an impedance between the PoC and the PCC}

Single-point measurements were shown in the previous section 4.2 to further no useful information on harmonic emission. Although exact time synchronisation will be possible, it will not make much difference when 10-min rms values are considered due to the principle of IEC 61000-4-30 aggregation, during this research a PQ instrument was used that is fully user configurable to allow for much shorter aggregation intervals. As the aggregation interval shortens, the importance of time synchronisation increases when direct comparison of measured values obtained by different instruments have to be done.

GPS synchronised measurements were taken at the Point of Common Coupling (PCC) representing $\boldsymbol{E}_{h 0}$ and at the PoC that represent $\boldsymbol{V}_{h}$ (as shown in Figure 4-1). Time stamping within an uncertainty $100 \mathrm{~ns}$ were obtained. This allowed direct comparison of data from different measurement points even if waveforms or 10/12 cycle measurement blocks were considered.

Harmonic emission criteria of the CIGRE/CIRED C4.109 method that requires identification of harmonic voltages where $\left|\boldsymbol{V}_{h}\right|>\left|\boldsymbol{E}_{h 0}\right|$ is now possible during the harmonic emission assessment of the PV plant under investigation. Figure 4-9 and Figure 4-10 shows the harmonic voltage rms values for the $5^{\text {th }}$ and $7^{\text {th }}$ harmonic respectively when making use of the requirement that $\left|\boldsymbol{V}_{h}\right|>\left|\boldsymbol{E}_{h 0}\right|$. In Figure 4-9, $E_{50}$ denotes the $5^{\text {th }}$ harmonic background network voltage. Similarly, in Figure $4-10, E_{70}$ denotes the $7^{\text {th }}$ harmonic background network voltage.

A voltage difference was also calculated and shown as a green line in Figure 4-9 and Figure 4-10. This green line literally represents harmonic emission (from the CIGRE/CIRED C4.109 principle).

Instances during the day when harmonic emission exist is clearly visible, but similar to section 4.2.1 instances exist as highlighted when $\left|\boldsymbol{V}_{h}\right|<\left|\boldsymbol{E}_{h 0}\right|$, a condition when the PV plant is helping the electrical utility to reduce the voltage harmonic under investigation. This is clearly not harmonic emission and those values should be discarded during the harmonic emission assessment. 


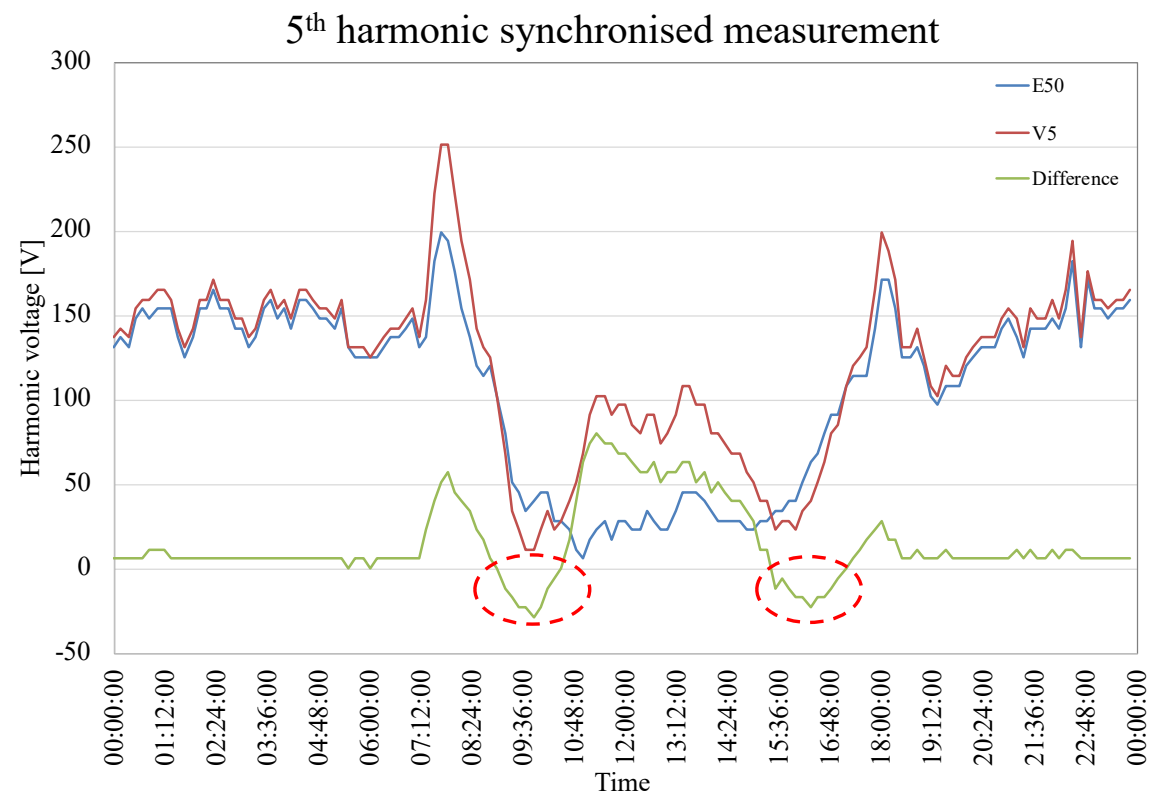

Figure 4-9. $5^{\text {th }}$ harmonic synchronised measurement voltage harmonics $\left|\boldsymbol{V}_{h}\right|>\left|\boldsymbol{E}_{h 0}\right|$

Harmonic emission at the $7^{\text {th }}$ harmonic order seems to be much simpler, or "fairer" considering the PV plant, as from Figure 4-10 the difference profile (green) is mostly positive. Instances where the difference is negative, is hardly noticeable.

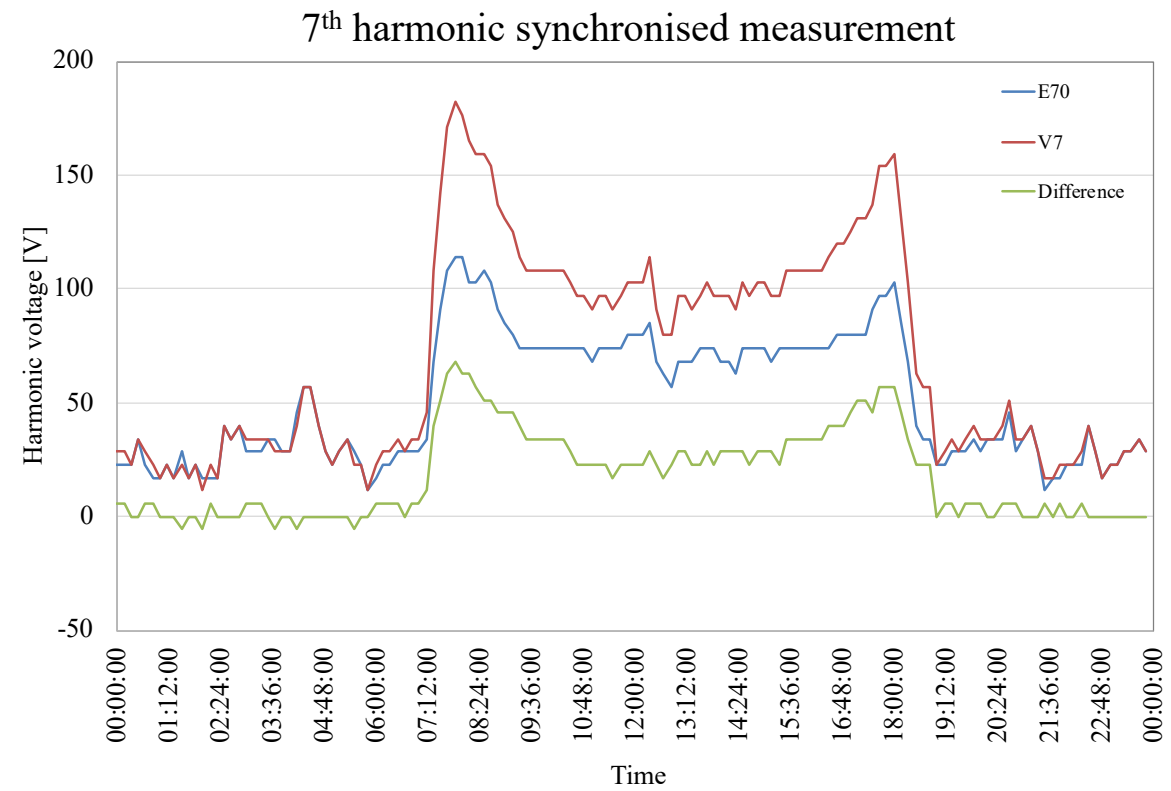

Figure 4-10. $7^{\text {th }}$ harmonic synchronised measurement voltage harmonics $\left|\boldsymbol{V}_{h}\right|>\left|\boldsymbol{E}_{h 0}\right|$

Harmonic voltage and current field data $\left(5^{\text {th }}\right.$ and $\left.7^{\text {th }}\right)$ could now be flagged for conditions considered as not harmonic emission. Results are shown in Figure 4-11 and Figure 4-12 respectively for the $5^{\text {th }}$ and $7^{\text {th }}$ harmonic order. 


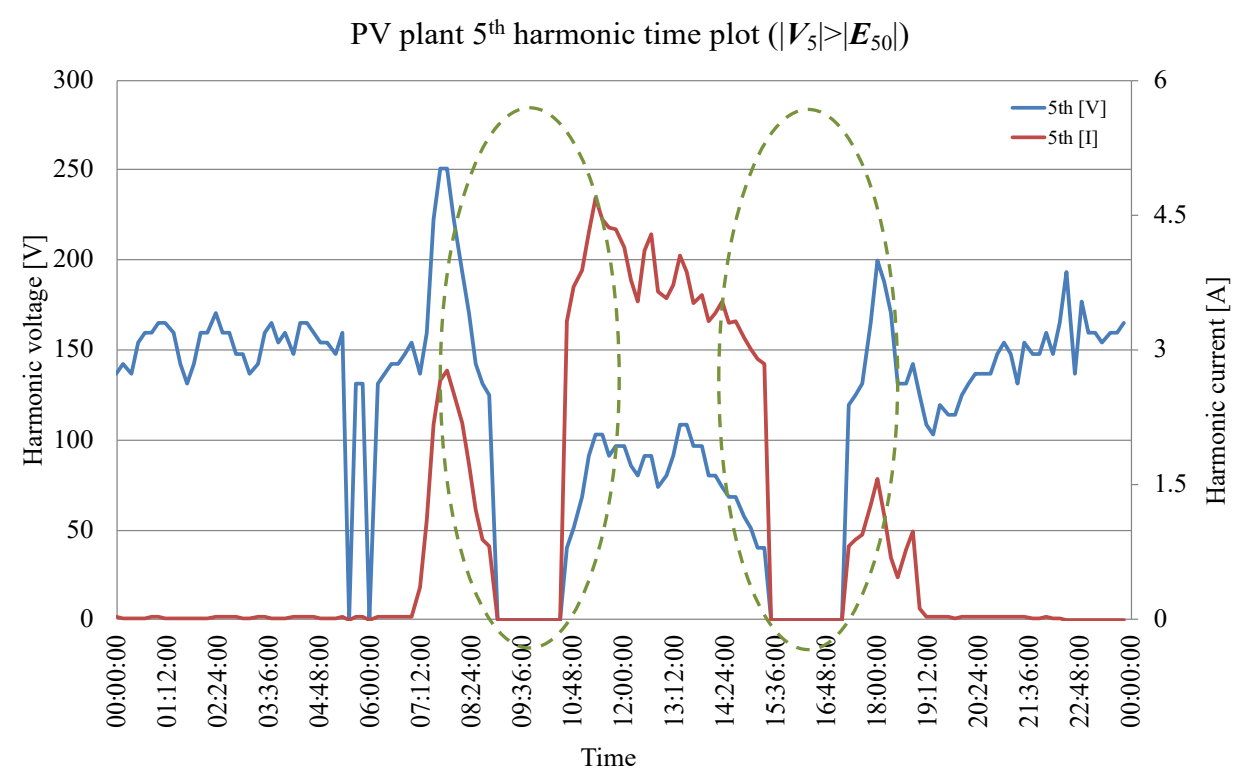

Figure 4-11. PV plant $5^{\text {th }}$ harmonic voltage and current time plot $\left|\boldsymbol{V}_{h}\right|>\left|\boldsymbol{E}_{h 0}\right|$

Figure 4-11 shows harmonic emission of the PV plant over a typical day at the $5^{\text {th }}$ harmonic order. Harmonic values that were removed coincide with instances when the harmonic voltage decreases when harmonic current increases as discussed in section 2.5.7 based on the raw data shown in Figure 4-2.

The above analysis again confirms that both the utility network and the PV plant are responsible for harmonic distortion at $5^{\text {th }}$ harmonic and that not all of the 10 -min rms values measured at the $\mathrm{PoC}$ are emission.

Figure 4-12 shows the $7^{\text {th }}$ harmonic voltage and harmonic current 10 -min rms values for a single day where the similar principle as applied to obtain Figure 4-11 was applied $\left(\left|\boldsymbol{V}_{h}\right|>\left|\boldsymbol{E}_{h 0}\right|\right)$. The PV plant is largely responsible for the harmonic emission, allowing for a much simpler analysis as encountered during the assessment of the $5^{\text {th }}$ harmonic.

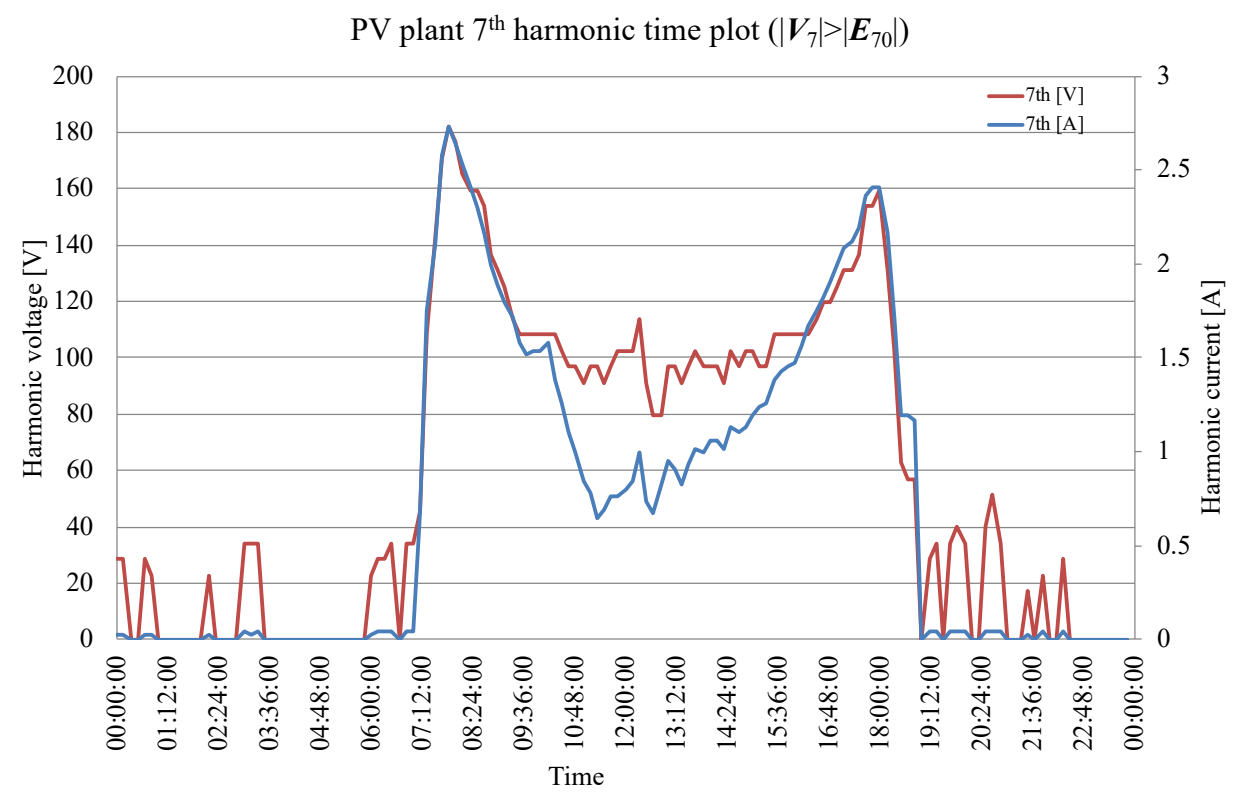

Figure 4-12. PV plant $7^{\text {th }}$ harmonic voltage and current time plot $\left|\boldsymbol{V}_{h}\right|>\left|\boldsymbol{E}_{h 0}\right|$ 
The best possible dataset when using 10-min rms values of voltage and current is now available by application of the synchronised measurements in this section. In the next section, the scatterplot approach of the CIGRE/CIRED C4.109 method will be repeated, and the new results compared to what was obtained in section 4.2 when only single-point measurements were available.

\subsubsection{Evaluation of CIGRE/CIRED C4.109 scatterplot method using synchronised measurement}

This section evaluates the CIGRE/CIRED C4.109 scatter plots when making use of a dataset now considered as reflecting harmonic emission $\left(\left|\boldsymbol{V}_{h}\right|>\left|\boldsymbol{E}_{h 0}\right|\right)$. The $5^{\text {th }}$ and $7^{\text {th }}$ voltage and current harmonic scatterplots are shown in Figure 4-13 and Figure 4-14 respectively. Presented data reflect only the discriminated data at the PV plant where the condition $\left|\boldsymbol{V}_{h}\right|>\left|\boldsymbol{E}_{h 0}\right|$ has been met.

A significant difference between Figure 4-13 and Figure 4-7 is that the two areas of datapoint grouping is now more distinct. What that practically means in terms of harmonic emission is not clear. One deficiency remains that absence of the impedance line for the PV plant (argued previously as mostly not available to the field engineer). At best, the results of Figure 4-13 remains qualitative.

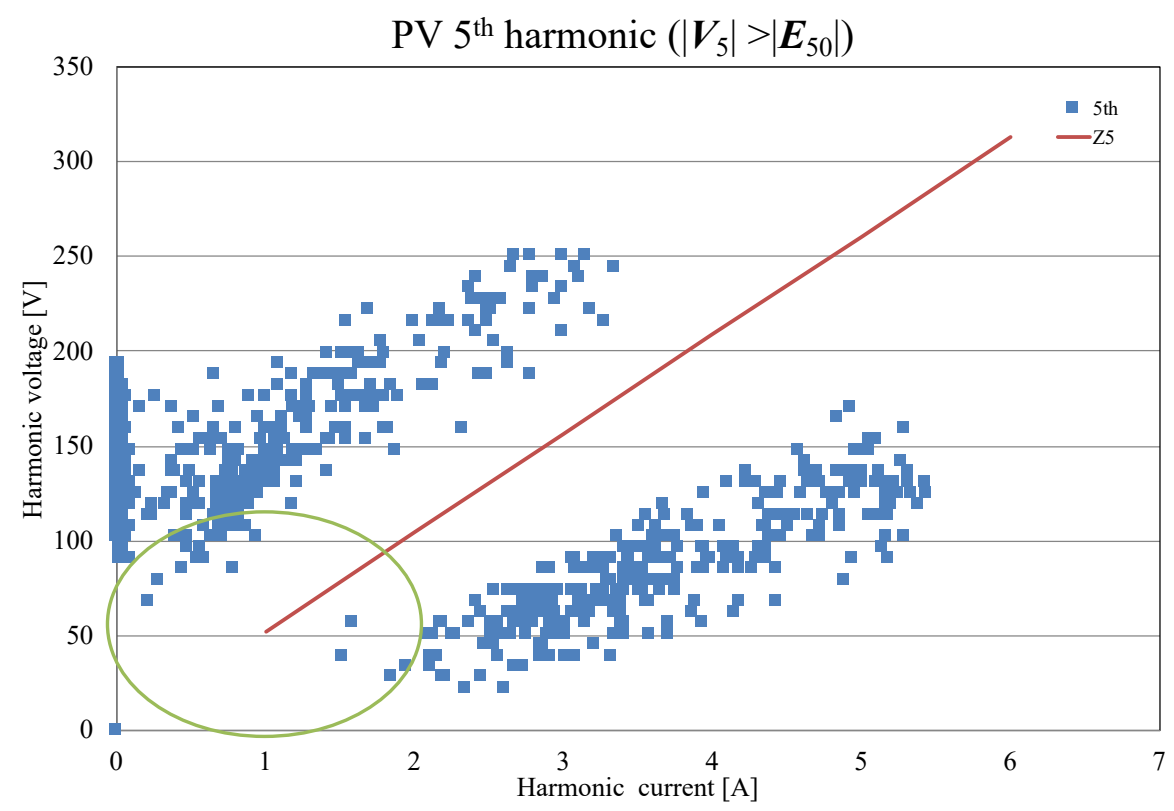

Figure 4-13. $5^{\text {th }}$ harmonic scatter plot at PV plant with $\left|\boldsymbol{V}_{5}\right|>\left|\boldsymbol{E}_{50}\right|$

Interpretation of Figure 4-14 seems much simpler as the datapoints concentrates around the utility impedance line and from the CIGRE/CIRED C4.109, the $7^{\text {th }}$ voltage harmonic measured at the PoC can be compared against the contracted values as they are indeed considered to be harmonic emission. 


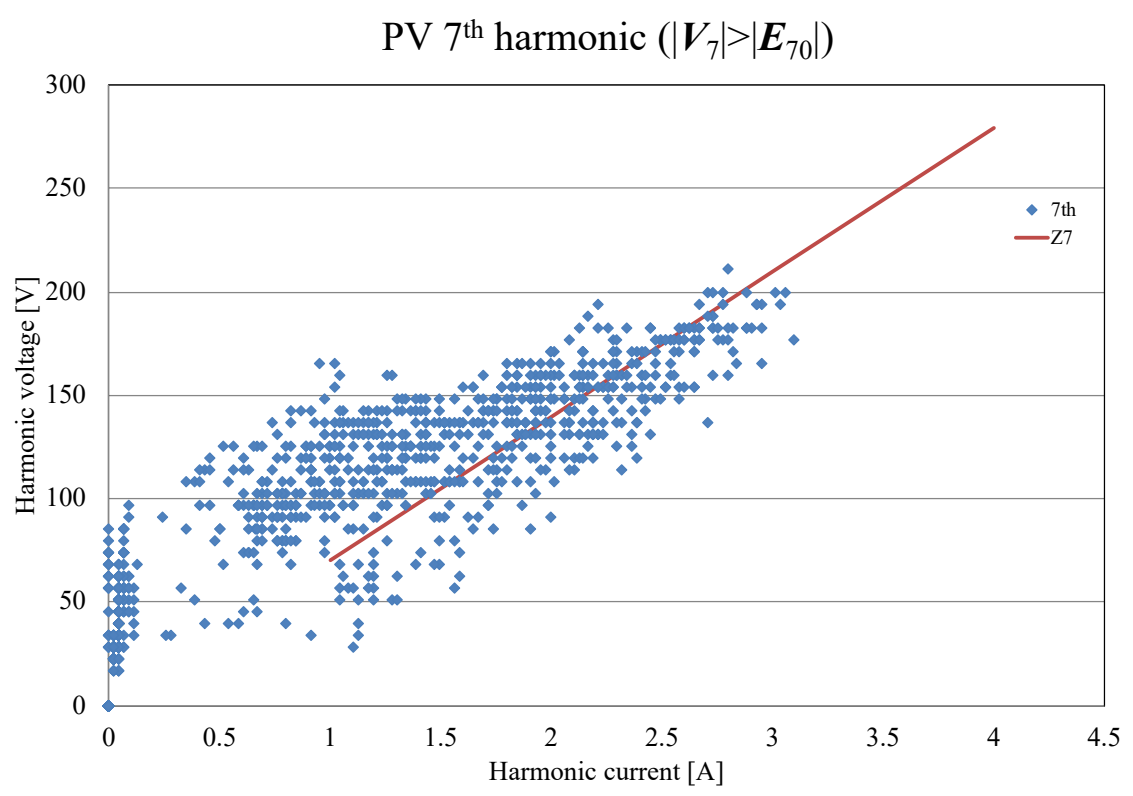

Figure 4-14. $7^{\text {th }}$ harmonic scatter plot at PV plant with $\left|\boldsymbol{V}_{7}\right|>\left|\boldsymbol{E}_{70}\right|$

\subsubsection{Harmonic emission using synchronised measurements}

Harmonic emission of the PV plant is redone in this section making use of the synchronised set of measurements, considered to possibly allow an improvement in the results (being "fairer" to the PV plant). A comparison of different emission calculation methods is done to evaluate the impact of harmonic emission discrimination on the end result.

The following principles are applied (to ensure comparable results all assessment is done at the PoC where grid code compliance is to be achieved):

1. Using the $95^{\text {th }}$ percentile of $10-\mathrm{min} \mathrm{rms} 5^{\text {th }}$ and $7^{\text {th }}$ harmonic current data for all data points at the $\mathrm{PoC}$ (no discrimination against harmonics regarded as emission and harmonic currents regarded as being sunk by the PV plant). This approach represents what most field engineers using single-point measurements will have to rely on as guided by the CIGRE/CIREC C4.109 method. It is referred to as the "Engineering method".

2. Using the $95^{\text {th }}$ percentile of the $5^{\text {th }}$ and $7^{\text {th }}$ harmonic current data at the PoC but where discrimination is applied through the condition that $\left|\boldsymbol{V}_{h}\right|>\left|\boldsymbol{E}_{h 0}\right|$ making use of synchronised measurements at the PoC and the PCC. Values deemed not to be emission are discarded.

3. Using the $95^{\text {th }}$ percentile of 10 -min rms harmonic current where the sign of the harmonic active power is positive (considered as emission as the sign fundamental frequency harmonic active power is negative). This method can be applied using only single-point measurements at the PoC of the PV plant.

Table 4-3 and Table 4-4 list the results as per the above mentioned assessment methods for the $5^{\text {th }}$ and $7^{\text {th }}$ harmonic orders. 
Table 4-3. Results of the $5^{\text {th }}$ harmonic emission assessment using 3 different methods

\begin{tabular}{|c|c|c|c|c|}
\hline \multicolumn{5}{|c|}{$5^{\text {th }}$ harmonic emission comparison } \\
\hline & Method & $95 \%$ I [A] & $\mathrm{Z}(\Omega)$ & $\mathrm{V}[\mathrm{V}]$ \\
\hline 1 & Engineering method & 4.4 & 52.1 & 229.2 \\
\hline 2 & $\left|\boldsymbol{V}_{\boldsymbol{h}}\right|>\mid \boldsymbol{E}_{\boldsymbol{h} \boldsymbol{0}}$ & 4.5 & 52.1 & 234.5 \\
\hline 3 & Active power & 4.3 & 52.1 & 224.0 \\
\hline
\end{tabular}

$5^{\text {th }}$ harmonic emission results using the various methods are similar with the $\left|\boldsymbol{V}_{h}\right|>\left|\boldsymbol{E}_{h 0}\right|$ method yielding the highest result. The second highest emission value is with all the data considered. Third highest is occupied by and the Active power method.

The similarity in the results is due to the use of a statistical approach in the $95^{\text {th }}$ percentile, which removed the "worst" $5^{\text {th }}$ percentile of data from the data set under consideration. With reference to Figure 4-11, it can be seen where discrimination has been applied, that harmonic emission values in the lower percentile (not considered to the in the "worst" $5^{\text {th }}$ percentile) were removed and not at the "worst" percentile values of the harmonic emission.

With reference to the harmonic emission current limits specified in Table 4-2, the PV plant does not comply for all of the harmonic emission assessment calculation methods considered.

Table 4-4. Results of the $7^{\text {th }}$ harmonic emission assessment using 3 different methods

\begin{tabular}{|c|c|c|c|c|}
\hline \multicolumn{5}{|c|}{$7^{\text {th }}$ harmonic emission comparison } \\
\hline & Method & $95 \%$ I [A] & $\mathrm{Z}(\Omega)$ & $\mathrm{V}[\mathrm{V}]$ \\
\hline 1 & Engineering method & 2.5 & 69.9 & 174.8 \\
\hline 2 & $\left|\boldsymbol{V}_{\boldsymbol{h}}\right|>\left|\boldsymbol{E}_{\boldsymbol{h} \boldsymbol{0}}\right|$ & 2.5 & 69.9 & 174.8 \\
\hline 3 & Active power & 2.3 & 69.9 & 160.8 \\
\hline
\end{tabular}

The "Engineering method" and the $\left|\boldsymbol{V}_{h}\right|>\left|\boldsymbol{E}_{h 0}\right|$ method yields the highest results with the active power method the lowest harmonic current emission value. Recall that the harmonic emission current is the main criteria that will be used for grid code compliance, which is why the focus is placed on harmonic current and not on harmonic voltage although both values are presented.

Considering a $95^{\text {th }}$ percentile harmonic current value may negate the advantages that discrimination of harmonic emission has on the final harmonic emission calculation as shown above.

While Table 4-3 shows no compliance to the $5^{\text {th }}$ harmonic emission limits irrespective of the applied method, Table 4-4 does highlight that the active power method may "prove" compliance as the $95^{\text {th }}$ percentile harmonic current is on the limit. Measurements of the harmonic emission values above are not without error [81]. In order for the results to have meaning a measurement uncertainty assessment must be conducted to indicate what the error margin is around the measured and calculated values. In the next section, a measurement uncertainty assessment is conducted for the measurement circuits used.

\subsection{Measurement uncertainty}

A contentious point is always when a RPP marginally exceeds the prescribed harmonic emission limits. Often an argument is made that the instrument transformers deployed at the RPP has a certain error margin and as such must be taken into consideration into the assessment criteria. While this is correct the "error" assessment does not stop at the instrument transformer but rather the whole measurement circuit must be evaluated to determine the measurement uncertainty as detailed in section 2.7 . 
Expanding on the harmonic emission assessment for the PV plant in Figure 4-1, Table 4-5 and Table 4-6 lists standard variances or "measurement errors" as obtained from the specification sheets of the equipment used in the measurement circuit. (Note that these values of instrument transformers apply to the power frequency. At harmonic frequencies, no additional information is needed. A field assessment of the higher frequency deviation of these results will be needed and mostly not available to field engineers as it is not possible to firstly remove the VT from service, and secondly, access to specialised calibration equipment is limited. It is therefore assumed that at the $5^{\text {th }}$ and $7^{\text {th }}$ harmonic, the deviation from the $50 \mathrm{~Hz}$ values are not significant.

Frequency response of instrument transformers is not linear [96]. A simplified uncertainty approach is needed in this research as characterisation of instrument transformers [96] is beyond the scope. For a detailed evaluation of measurement uncertainty across the harmonic spectrum, the influence of frequency response on measurement errors should be used.

Table 4-5. List of components within the voltage measurement chain

\begin{tabular}{|c|c|c|}
\hline Source of Uncertainty & $\begin{array}{c}\text { Standard uncertainty } \boldsymbol{u}\left(\boldsymbol{x}_{\boldsymbol{i}}\right) \\
\text { [magnitude] }\end{array}$ & $\begin{array}{c}\text { Standard uncertainty } \boldsymbol{u}\left(\boldsymbol{x}_{\boldsymbol{i}}\right) \text { [angle, } \\
\text { degrees] }\end{array}$ \\
\hline PQ instrument [49] & $0.1 \%$ & 0.4 \\
\hline Voltage transformer & $1 \%$ & 0 \\
\hline VT supply cable & $0.20 \%$ & 0 \\
\hline
\end{tabular}

Table 4-6. List of components within the current measurement chain

\begin{tabular}{|c|c|c|}
\hline Source of Uncertainty & $\begin{array}{c}\text { Standard uncertainty } \boldsymbol{u}\left(\boldsymbol{x}_{\boldsymbol{i}}\right) \\
\text { [magnitude] }\end{array}$ & $\begin{array}{c}\text { Standard uncertainty } \boldsymbol{u}\left(\boldsymbol{x}_{\boldsymbol{i}}\right) \\
\text { [angle, degrees] }\end{array}$ \\
\hline PQ instrument [49] & $0.1 \%$ & 0.4 \\
\hline $\begin{array}{c}\text { Clamp-on current transducer } \\
\text { (obtained from data sheet) }\end{array}$ & $2.0 \%$ & 1.5 \\
\hline $\begin{array}{c}\text { Current transformer [97] } \\
\text { (taken at 20\% burden) }\end{array}$ & $0.4 \%$ & 0.3 \\
\hline Current transformer supply cable & $0.1 \%$ & 0 \\
\hline
\end{tabular}

Accuracy levels for instrument transformers may also be obtained from the IEC 61000-4-7 [74] and from IEC 61869-103 [87] if the instrument transformer is certified to comply with these standards. The latter document specifies accuracy levels of harmonics up to the $13^{\text {th }}$ harmonic for both rms and phase displacement.

A simplified version of Figure 2-29 is shown in Figure 4-15 to aid the discussion on measurement uncertainty. Standardised instrument and transducer equipment are used in the PoC and PCC substations as required by the electrical utility. 


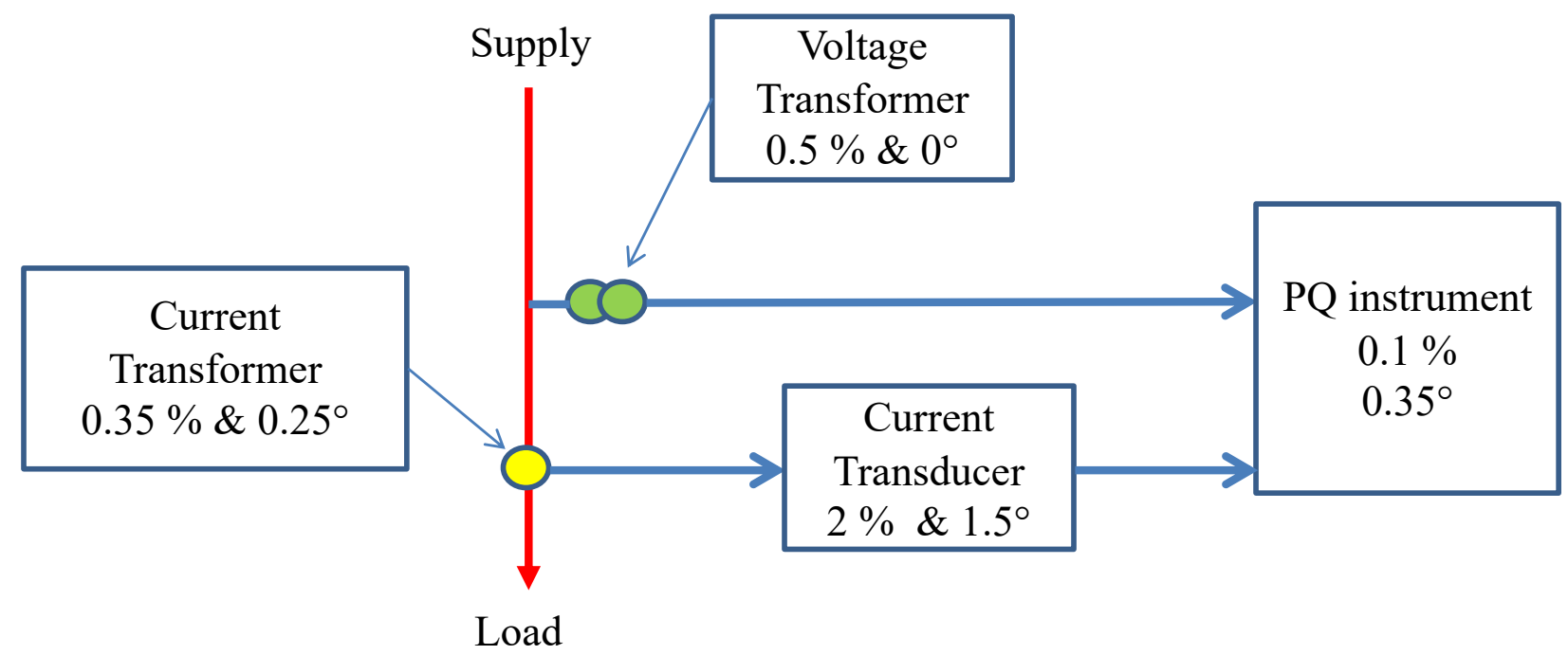

Figure 4-15. Simplified measurement circuit and the uncertainty error for each component contributing to the measurement chain.

Application of (27) while assuming a rectangular error distribution (meaning the error is constant across measurement range in term of magnitude) and input values listed in Table 4-5 and Table 4-6 for the voltage and current measurement chains, results of the Combined Standard Uncertainty (CSU) is listed in Table 4-7.

Confidence in the CSU is further provided by calculating the Expanded Combined Standard Uncertainty (ECSU), which accounts for the probability that the measurand will be within the selected error distribution curve. Expanded CSU for a rectangular distribution is calculated for a 95\% coverage with the results listed in Table 4-8.

Table 4-7. Combined standard uncertainty for measurement circuit in Figure 4-15

\begin{tabular}{|l|c|c|}
\hline Parameter & Magnitude & Phase angle \\
\hline Harmonic current & $1.2 \%$ & $0.9^{\circ}$ \\
\hline Harmonic voltage & $0.1 \%$ & $0.2^{\circ}$ \\
\hline
\end{tabular}

Table 4-8. Expanded combined standard uncertainty for the measurement circuit in Figure 4-15.

\begin{tabular}{|l|c|c|}
\hline Parameter & Magnitude & Phase angle \\
\hline Harmonic current & $1.9 \%$ & $1.5^{\circ}$ \\
\hline Harmonic voltage & $0.2 \%$ & $0.3^{\circ}$ \\
\hline
\end{tabular}

With the measurement uncertainty budget calculated, the results must be applied to the actual measurand which in this case is the harmonic current emission that will be used for grid code compliance.

Using the harmonic current emission results listed in Table 4-3 and Table 4-4 an uncertainty band is established around the current harmonic emission results by adding and subtracting the ECSU listed in Table 4-8. Although the result presented is the $95^{\text {th }}$ percentile, the uncertainty assessment can be applied to all measured results.

Table 4-9 and Table 4-10 list the results of the harmonic emission assessment calculations. 
Table 4-9. Results of the $5^{\text {th }}$ harmonic emission assessment using 3 different method and with measurement uncertainty applied.

\begin{tabular}{|c|c|c|c|c|}
\hline \multicolumn{5}{|c|}{$\boldsymbol{5}^{\text {th }}$ harmonic emission comparison } \\
\hline & Method no & $95 \%$ I [A] & $-1.90 \%$ & $1.90 \%$ \\
\hline 1 & Engineering method & 4.4 & 4.32 & 4.48 \\
\hline 2 & $\left|\boldsymbol{V}_{\boldsymbol{h}}\right|>\left|\boldsymbol{E}_{\boldsymbol{h} \boldsymbol{0}}\right|$ & 4.5 & 4.41 & 4.59 \\
\hline 3 & Active power & 4.3 & 4.22 & 4.38 \\
\hline
\end{tabular}

Table 4-10. Results of the $7^{\text {th }}$ harmonic emission assessment using 3 different method and with measurement uncertainty applied.

\begin{tabular}{|c|c|c|c|c|}
\hline \multicolumn{5}{|c|}{${\boldsymbol{T}^{\text {th }}}^{\mathbf{5} \text { harmonic emission comparison }}$} \\
\hline & Method no & $95 \% \mathrm{I}[\mathrm{A}]$ & $-1.90 \%$ & $1.90 \%$ \\
\hline 1 & Engineering method & 2.5 & 2.45 & 2.55 \\
\hline 2 & $\left|\boldsymbol{V}_{\boldsymbol{h}}\right|>\left|\boldsymbol{E}_{\boldsymbol{h} \boldsymbol{0}}\right|$ & 2.5 & 2.45 & 2.55 \\
\hline 3 & Active power & 2.3 & 2.26 & 2.34 \\
\hline
\end{tabular}

In practice the presented values listed above shows a realistic view of what the actual measurements can be, defined within a narrow band around the resultant measurand. If the harmonic active power method was allowed for grid code compliance in South Africa, this method would have resulted in the PV plant being deemed compliant for the $7^{\text {th }}$ harmonic current as the lower value is below the $95^{\text {th }}$ percentile value. In contrast, the utility may argue that the harmonic current emission value is exceeding the upper of the uncertainty band value when benchmarked against the prescribed limit.

The nature of measurement uncertainty is that a value (measurand) can be above or below the measurement uncertainty band. In the spirit of a fair and equitable harmonic emission assessment, the benefit should be given to the RPPs especially if the utility can contain their global harmonic voltage distortion at the $\mathrm{PoC}$ or PCC within regulatory limits. Application of measurement uncertainty assessment could further prevent wasteful expenditure on PQ mitigation equipment when there are marginal contractual limit exceedances, as it may be shown that these marginal exceedances falls within the measurement uncertainty band and can thus not be deemed as not be taken as absolute.

\subsection{Conclusion}

Harmonic emission methodologies that make use of rms values of harmonic voltage and current phasors were evaluated using field data obtained at a renewable energy source.

Grid code compliance is absolute and incorrect harmonic emission assessments may lead to wasteful expenditure on mitigation equipment or disputes between DSOs and RPP owners. A pragmatic and fair approach to harmonic emission assessment is required.

The aim of harmonic emission assessment is to account for only the values which are emitted from the non-linear load, in this case, a PV plant. In using rms values of harmonic phasors during the assessment of emission, the normal approach regard all of the values as being emitted from the PV plant.

No discrimination is normally done between those harmonic currents being emitted and those being absorbed or sunk by the RPP. This is an incorrect assumption as there are instances in time when a PV plant is of benefit to the utility by the PV plant reducing the voltage harmonic emission at the PoC. This is a time-dependent phenomenon as later during the day, the harmonic current phasor can change direction and indeed become emission. 
In an attempt to discriminate between harmonic currents being absorbed or emitted, the harmonic active power method was applied to identify 10-min rms harmonic current values to be regarded as emission (increasing the network harmonic voltage). Harmonic active power was found to not be able to fully explain all conditions. It confirmed the dilemma of harmonic active power not being conclusive when analysing single-point measurements in a network where more than one source of harmonic emission exist.

One of the statutory harmonic emission assessment methods prescribed in the South African grid code, the CIGRE/CIRED C4.109 scatter plot method, was applied to a PV plant considering the rms values of the $5^{\text {th }}$ and $7^{\text {th }}$ harmonic voltage and current phasors. The results of the analysis show that the $7^{\text {th }}$ harmonic voltage emission into the network is the result of PV plant injecting $7^{\text {th }}$ harmonic currents, concluding that the CIGRE/CIRED C4.109 scatter plot method produced a fair result.

Analysis of the $5^{\text {th }}$ harmonic voltage and current indicated that harmonic voltage emission into the network is due to both the PV plant and the utility network. The CIGRE/CIRED C4.109 scatter plot method could not produce conclusive results on the assessment of harmonic emission at the $5^{\text {th }}$ harmonic order.

Additional measurements were done using synchronised data across an impedance to evaluate the usefulness of harmonic active power towards improving the CIGRE/CIRED C4.109 scatter plot method. Synchronised data at more than one point of measurement also indicate when the harmonic voltage at the PV plant is higher than the harmonic voltage of the upstream PoC, regarded as the fundamental principle of harmonic emission.

Synchronised data allowed discrimination by removing harmonic current data that do not contribute to an increase in network harmonic voltage. This improved the scatterplots but could not fully discriminate between network and PV plant contribution to the $5^{\text {th }}$ harmonic voltage when applying the CIGRE/CIRED C4.109 scatter plot method. At the $7^{\text {th }}$, it was reconfirmed that the $\mathrm{PV}$ plant is responsible for the harmonic voltage distortion at the PoC.

Harmonic emission assessment using a 10-min rms value measured at a single point may result in inconclusive results as highlighted in this chapter using field measurements at a PV plant. By expanding the measurement campaign through synchronised and coherent measurements at different points on the network, a discriminatory approach can be considered.

Harmonic emission assessment using 3 different approaches [15], [98] to highlight that different harmonic emission results are obtained when using different methods. Reason why the harmonic emission results of the different methods for both the $5^{\text {th }}$ and $7^{\text {th }}$ harmonic current values were similar are due to the use of the $95^{\text {th }}$ percentile. It is therefore important to be not absolute and apply a compatibility approach that do not take into account the plant performance over $100 \%$ of the time.

Harmonic emission assessment requires evaluation of measurement uncertainty assessment as not all harmonic current values measured during a field study cannot be acquired with sufficient certainty. Measurement uncertainty is very important when the RPP marginally exceeds the allowable harmonic emission limit. Accuracy of instrumentation transformers are limited in bandwidth and needs to be considered. 


\section{CHAPTER 5: Assessment of harmonic emission using aggregated harmonic phasors: engineering application}

\subsection{Introduction 9}

In the previous chapter a harmonic emission assessment was conducted for a PV plant undergoing grid code compliance as guided by the South African grid code. Short comings in the assessment process was highlighted, which mainly focused around the use of a 10-min rms value to prove or calculate the harmonic emission. The limited harmonic emission assessment methods were also applied, and it was shown that not all of the results are conclusive.

Most of the harmonic emission assessment methods discussed in section 2.5 require the measurement of harmonic phasors [13], [14], but continuous measurement of harmonic phasors (updated every $200 \mathrm{~ms}$ ) results in a large volume of data that becomes cumbersome to process when the measurement are taken for more than 24 hours.

IEC 61000-4-30 aggregation principles reduce $200 \mathrm{~ms}$ (10/12 cycle) data sets to, for example 10min rms values, as known PQ standards base compatibility criteria on 10-min rms values. Retention of the harmonic phase angle information after a Fourier analysis results in aggregated harmonic phasors which can reduce the data set for harmonic emission assessment.

The first contribution to the improvement of engineering knowledge on harmonic emission in this chapter address the deficiency of the CIGRE/CIRED C4.109 method. It was shown in the previous chapters to be ineffective in a distribution grid where harmonic emission by renewable generation continuously change. More useful results are obtained when aggregated harmonic phasors are used and not only 10 -min rms values.

Secondly, the principle of assessing what range of harmonic phase angles will increase the voltage harmonic phasor in the supply network (emission) and what range of harmonic phase angles will decrease the voltage harmonic phasor in the supply network (sink), is used in deriving a practical Range of Secondary Cancellation (RSC) for a specific harmonic order. This RSC principle is then demonstrated to significantly improve the assessment of harmonic emission as needed during a grid code evaluation study at a large distribution grid-connected PV plant.

A passive filter relies on the network impedance to be effective in containing a specific harmonic order by creating a low impedance path (for example) for a harmonic current phasor at the harmonic order of interest. In a practical network, this network impedance can change, or even the impedance elements in the filter, affecting the filtering performance. In conclusion, the RSC principle is shown to assess the performance of a passive harmonic filter.

\subsection{Aggregation of harmonic phasors}

Aggregation of harmonic phasors are done according to the principles of the IEC 61000-4-30 PQ measurement standard. The principles as described in section 2.6.1 result in rms aggregated values, where the harmonic phase angle is neglected. Doing the same with harmonic phase angles, allows a novel approach in retaining harmonic phasor information required for harmonic emission assessment [73].

Fourier analysis of the IEC 61000-4-30 10/12 cycle block of data (200 ms for $50 \mathrm{~Hz}$ ) produce harmonic phasors [74], [80] as shown in (33) below:

\footnotetext{
${ }^{9}$ One peer-reviewed conference paper was published [109] .
} 


$$
\boldsymbol{Y}_{h}=Y_{h} \angle \varphi_{h}
$$

Where,

$Y_{h}: \quad$ the $10 / 12$ cycle $(200 \mathrm{~ms})$ rms value of the harmonic voltage or current phasor at harmonic $h$.

$\varphi_{h}: \quad$ the $10 / 12$ cycle $(200 \mathrm{~ms})$ harmonic voltage or current phase angle at harmonic $h$ Aggregation of harmonic phasors is obtained by (34) below:

$$
\boldsymbol{Y}_{\text {agg,h }}=Y_{\text {agg,h }}<\varphi_{\text {agg }, h}
$$

Where;

$$
Y_{\text {agg }, h}=\left(\frac{1}{n} \sum_{i=1}^{n} Y_{H, h, i}^{2}\right)^{\frac{1}{2}}
$$

Aggregated rms values of the harmonic phasor $\left(Y_{a g g, h}\right)$ at harmonic order $h$ is derived from the magnitude of 10/12-cycle block phasors as required by the IEC 61000-4-30 principle. $Y_{\text {agg, }}$ may be replaced by $I_{a g g, h}$ (harmonic current) or $U_{a g g, h}$ (harmonic voltage) where $n$ is the number of harmonic phasors to be aggregated. For example, $n=15$ for a 3 -sec aggregation interval and $n=$ 300 for a 1-min aggregation interval.

The aggregated phase angle is obtained by (36).

$$
\varphi_{a g g, h}=\arg \left(\sum_{i=1}^{N} \boldsymbol{Y}_{H, h, i}\right)
$$

Where $N$ is the number of harmonic phasors to be summated.

Consideration to the stability of the harmonic phase angle is needed when harmonic phase angles have to be aggregated. A qualitative, albeit subjective, criteria needs to be defined for when a harmonic phasor is regarded as "sufficiently stable/time independent". Practically, it means that during the period of observation, the changes in the both the magnitude and phase angle of the harmonic phasor under consideration, are contained within this subjective criterion. In [73], [80] it is stated that if the phase angle is considered as the criterion on harmonic phasor change, then a change less than $10 \%$ over that period is regarded as a harmonic phase angle to be sufficiently "prevalent" or stable. Quantifying the variation of the aggregated phasor termed "level of prevalence" is calculated by a "prevailing ratio" $P R_{a g g, h}$ that in principle assess the grouping or clustering of harmonic phasors during the period of evaluation as defined in (37) below.

$$
P R_{a g g, h}=\frac{\left|\sum_{i=1}^{n} \boldsymbol{Y}_{H, h, i}\right|}{\sum_{i=1}^{n}\left|\boldsymbol{Y}_{H, h, i}\right|}
$$

Prevalence levels were derived from extensive probabilistic studies and defined as follows [73], [80]:

- High prevalence when $P R_{a g g, h}$ is $\geq 0.95$. 
- Medium prevalence when $0.95>P R_{a g g, h} \geq 0.89$

- Low prevalence when $0.89>P R_{a g g, h} \geq 0.8$

- $\quad$ No prevalence when $P R_{a g g, h}<0.8$

From the above, if the prevalence level is less than 0.8 , then the aggregated harmonic phase angle is regarded as useless. Whether this subjective conclusion is directly applicable to a distribution network, is investigated in this chapter as the principles above are from data obtained in mostly residential networks [73], [80].

A prevailing phasor is calculated in (38) below when the test for prevalence is passed. Equation (34) and (38) are the same, except that the number of data points used to reflect the period of aggregation in (38) is regarded as "sufficiently" stable for the harmonic phasor.

$$
\boldsymbol{Y}_{p r v, h}=Y_{p r v, h} \angle \varphi_{p r v, h}
$$

Where;

$$
Y_{p r v, h}=\left(\frac{1}{n} \sum_{j=1}^{n} Y_{a g g, h, j}^{2}\right)^{\frac{1}{2}}
$$

and

$$
\varphi_{p r v, h}=\arg \left(\sum_{j=1}^{n} \boldsymbol{Y}_{a g g, h, j}\right)
$$

Application of the aggregated harmonic phasor in harmonic emission assessment is considered next in context of a distribution network.

\subsection{Opportunity for the prevailing phase angle}

Results in the prevailing harmonic phasor (phase angle) is reported in [73] from measurements obtained in a low voltage (LV) network. The goals were firstly to compare the harmonic performance of the German network to a few other countries and secondly, to evaluate the interaction between different types of harmonic producing loads such as PV and electric vehicles [99], [100].

In this thesis, field data was obtained by implementing the aggregation principles of the previous section in the firmware of a PQ measurement instrument certificated to be IEC 61000-4-30 Edition 3 Class A compliant. A time aggregation of 1-min was selected for the aggregated harmonic phasors [73]. This PQ instrument was installed at a large (5 MVA, $11 \mathrm{kV})$ PV plant as shown in Figure 5-1. The goal was to assess harmonic emission against the requirements of the grid code [1] agreement between the utility who owns the distribution network and the owner of the PV plant.

Note that the principles of measurement uncertainty assessment of the whole measurement chain was presented in section 4.4 and is not presented here. It does not detract of the importance thereof.

\subsubsection{CIGRE/CIRED C4.109 application}

The CIGRE/CIRED C4.109 scatter plot method aims at assessing the major contributor of the harmonic distortion at a $\mathrm{PoC}$ between non-linear load and the upstream network. It cannot 
quantitatively distinguish between the harmonic distortion contributed by the upstream supply network and the known harmonic distortion source but can reveal from a qualitative point of view the dominance of either one as discussed in section 2.5.7.

For the purpose of demonstration, analyses of the PV plant shown in Figure 5-1 for this section will be done using the $5^{\text {th }}$ and $7^{\text {th }}$ harmonic orders only using the CIGRE/CIRED C4.109 method.

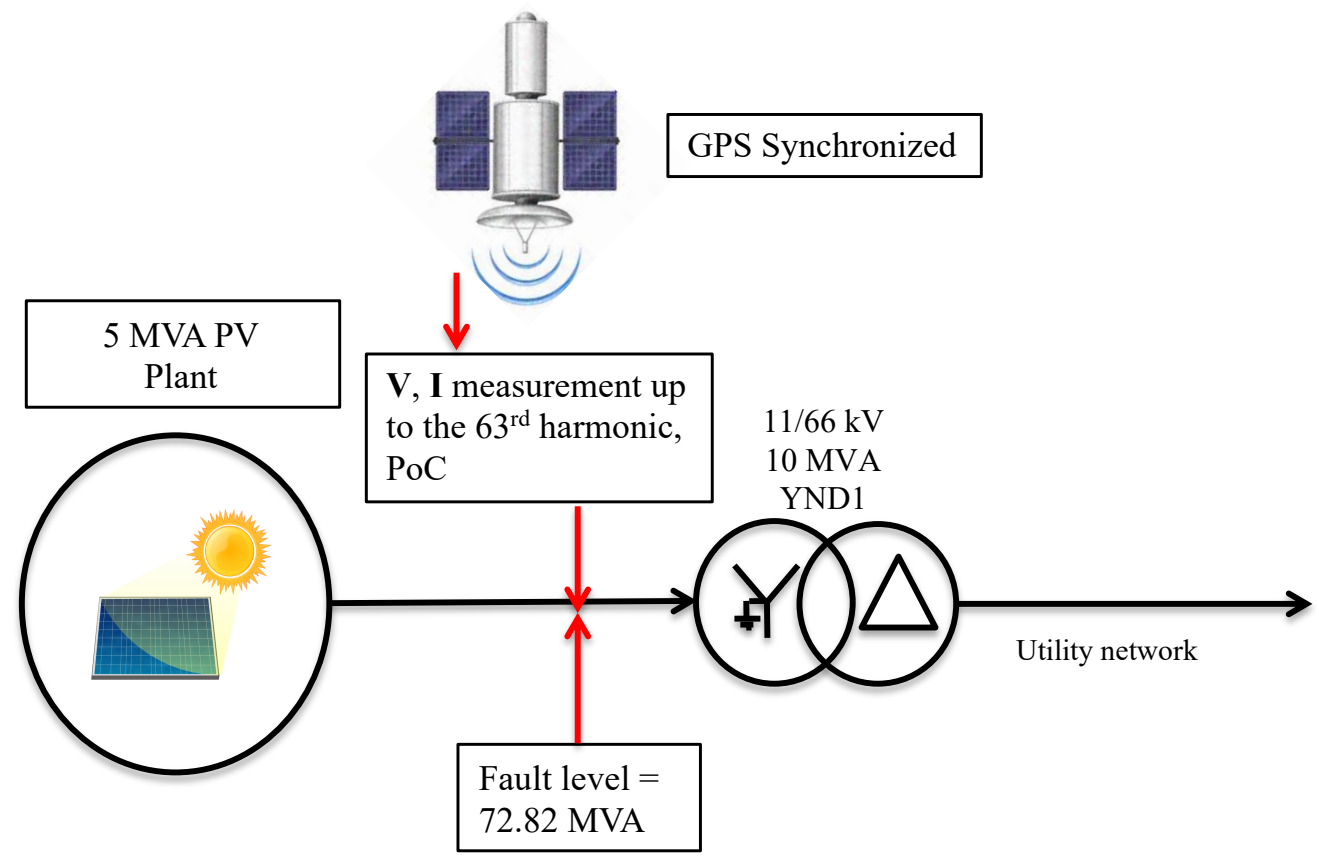

Figure 5-1. Network diagram for PV plant

Harmonic voltage and current 10-min rms data was recorded at the PoC of the PV plant in Figure 5-1over a 7 day period as per the requirements of the South African grid code. The PQ recorder that was used for the measurements, is IEC61000-4-30 class A complaint. Figure 5-2 and Figure 5-3 shows the $5^{\text {th }}$ and $7^{\text {th }}$ harmonic CIGRE/CIRED scatter plot of the measured harmonic voltage and harmonic current rms values respectively.

The harmonic impedance of the supply network at the PoC was obtained from the network model in use by the utility. The harmonic impedance of the PV plant was determined by application of (41) below. Alternatively, the rating of the coupling transformer at the PV plant may be used for the harmonic impedance [72]. Note that this calculation will result in a linear extrapolation of the PV plants harmonic impedance and does not take reactive compensation equipment into consideration. It is only used here as the PV plants harmonic impedance information was not available.

$$
Z_{h}=\frac{V^{2}}{S} \cdot h
$$

Where;

$Z_{h}$ : calculated harmonic impedance of the PV plant

$V: \quad$ voltage level at the point of connection

$S: \quad$ maximum power rating of the PV plant

$h: \quad$ the harmonic order 
$5^{\text {th }}$ Harmonic voltage and current scatter plot

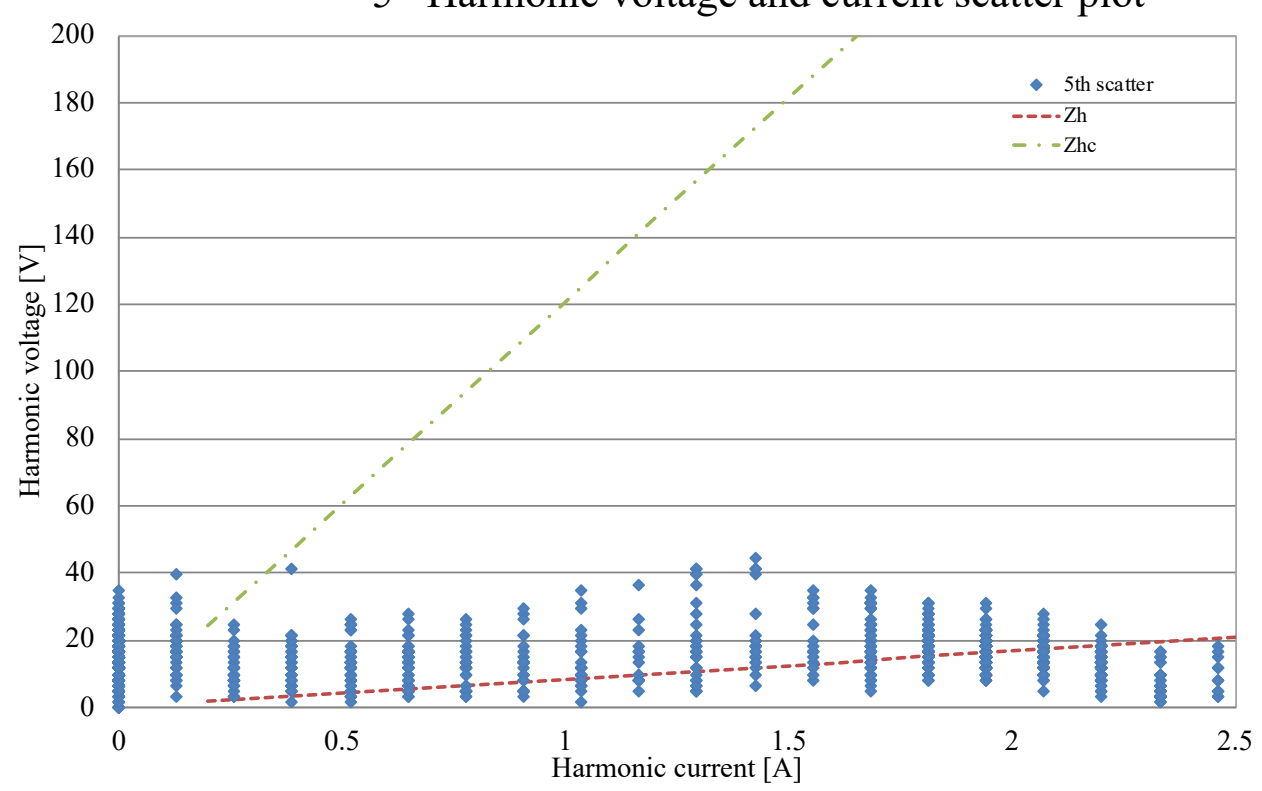

Figure 5-2. $5^{\text {th }}$ harmonic voltage and current scatter plot for $P V$ plant

Figure 5-2 shows that the PV plant dominates distortion at the $5^{\text {th }}$ harmonic order as the rms values of voltage and current concentrate around the network impedance locus.

Figure 5-3 presents the $7^{\text {th }}$ harmonic voltage and current CIGRE/CIRED scatter plot.

$7^{\text {th }}$ Harmonic voltage and current scatter plot

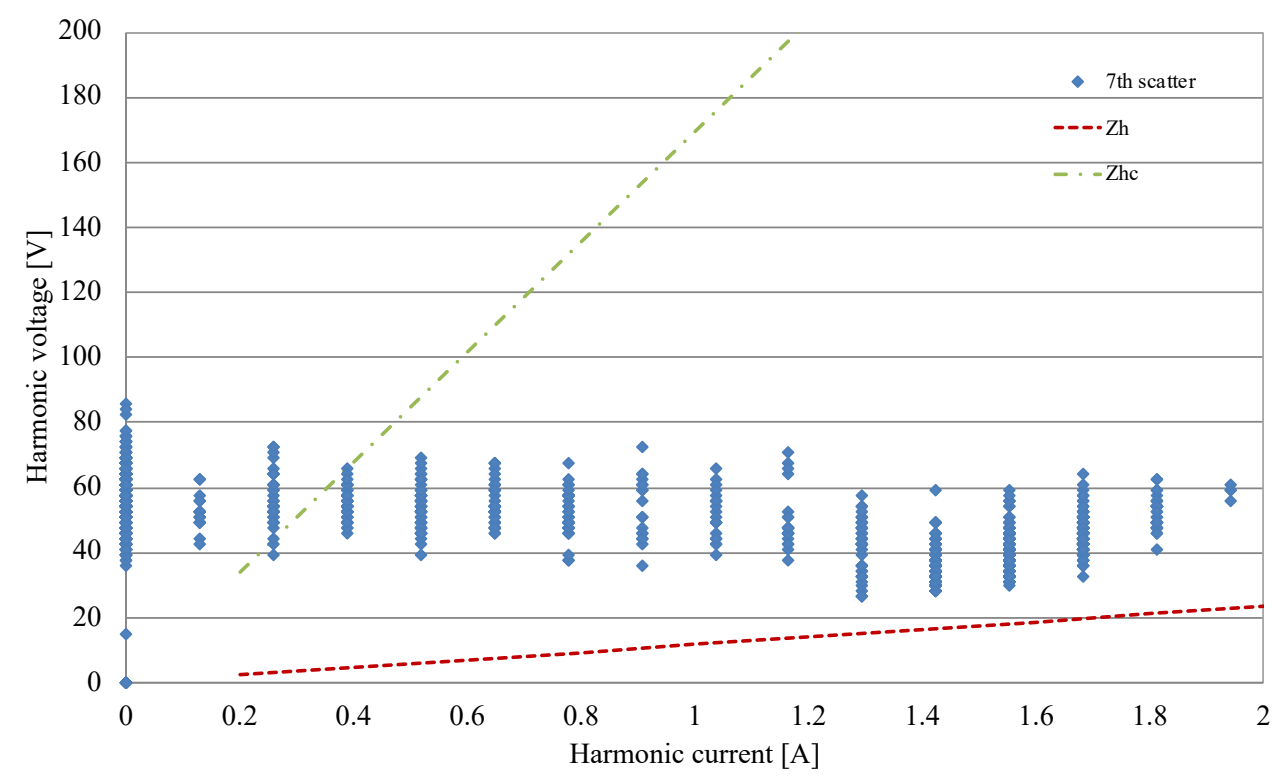

Figure 5-3. $7^{\text {th }}$ harmonic voltage and current scatter plot for $P V$ plant

Figure 5-3 confirms the qualitative constraint in the application of the CIGRE/CIRED C4.109 scatter plot method. Both the utility network (upstream) and the PV plant contribute to harmonic distortion at the $7^{\text {th }}$ harmonic order. Data points are distributed between the two impedance loci, resulting in no added value towards the assessment of harmonic emission at the $7^{\text {th }}$ harmonic order. 
No conclusion can be made for the purpose of grid code compliance assessment on the emission values contracted between the utility and the PV plant owner.

In the next section, field data consisting of aggregated harmonic phasors is analysed to better understand why harmonic emission assessment for grid code compliance [1] cannot be done using the CIGRE/CIRED C4.109 method.

\subsubsection{Analysis of harmonic field data based on aggregated harmonic phasors}

With the CIGRE/CIRED C4.109 harmonic emission assessment showing inconclusive results that can easily be challenged, an improved harmonic emission assessment methodology is required. In this section aggregated harmonic measurements taken at the same PV plant as discussed in the previous section is analysed to gain a better understanding into harmonic emission assessment using aggregated harmonic phasors.

All data presented in this section and below was measured with the PQ instrument deployed in Figure 5-1 but configured to also record the aggregated harmonic phasors with a 1-min time interval. The 1-min time interval was guided by previous studies that made use of aggregated harmonic phasors [73]. Figure 5-4 and Figure 5-5 presents only the rms components of the 1-min aggregated phasors for the $5^{\text {th }}$ and $7^{\text {th }}$ harmonic component respectively.

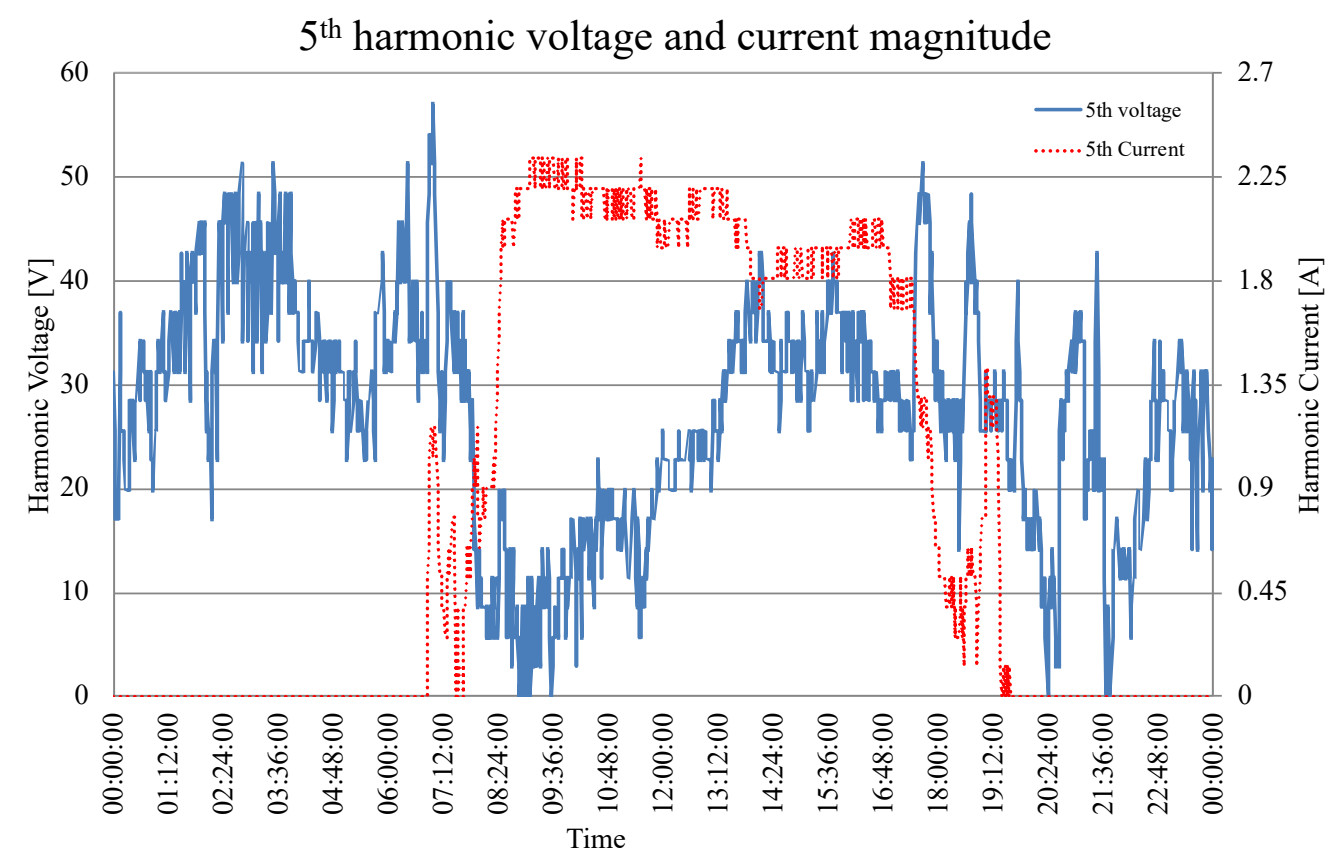

Figure 5-4. $5^{\text {th }}$ harmonic voltage and current time plot

By comparison of voltage and current values shown in Figure 5-4 the following is observed. During the periods of low production in the morning and the evening, the $5^{\text {th }}$ harmonic current and the $5^{\text {th }}$ harmonic voltage shows an inverse correlation, as it can be seen that the harmonic voltage reduces for an increase in the harmonic current. While the $5^{\text {th }}$ harmonic current remains high during the day, the harmonic voltage profile does not remain low as in the morning period. It is not known what the cause is of the increase in the harmonic voltage after the 12:00 time stamp. Can this increase in harmonic voltage be due to the harmonic emission of the PV plant or due to the changes in the upstream network? With only an rms value at hand the reason for the change is not easily identified as previously discussed in section 4.2.

The $7^{\text {th }}$ harmonic voltage and current profile in Figure 5-5 does not follow the same pattern as with the $5^{\text {th }}$ harmonic in Figure 5-4. The $7^{\text {th }}$ harmonic current does not determine the harmonic voltage 
at the PoC, indicating that the PV plant is not the dominant contributor to harmonic distortion at the PoC. Based on only rms values, there appears to be inverse correlation between the $7^{\text {th }}$ harmonic voltage and harmonic current. The slow increase in harmonic voltage during the day, whilst the harmonic current remains relatively constant, is not seen for the $7^{\text {th }}$ harmonic.

The CIGRE/CIRED C4.109 harmonic emission analysis in the previous section highlighted that the $7^{\text {th }}$ harmonic analysis was inconclusive. Harmonic emission at the $7^{\text {th }}$ harmonic order cannot be unambiguously assigned to the PV plant from the measurements at hand. Grid code compliance assessment cannot be based on this set of data shown in Figure 5-5. During field studies this is unfortunately the typical data set that will be collected as the per grid code compliance process detailed in [1].

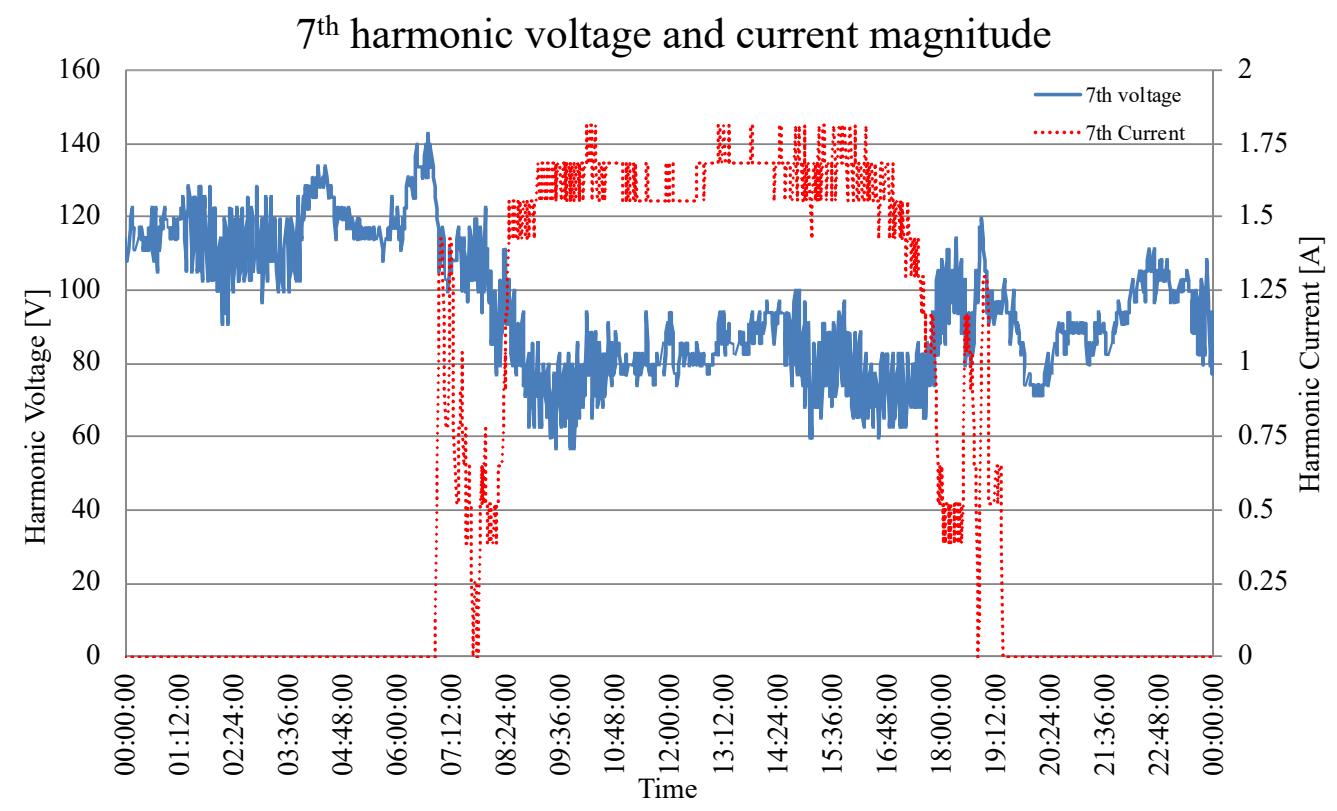

Figure 5-5. $7^{\text {th }}$ harmonic voltage and current time plot

Analysis of the prevailing phase angle for the $5^{\text {th }}$ and $7^{\text {th }}$ voltage harmonics reveal additional information in Figure 5-6 as demonstration of how harmonic phase angle can improve the understanding of harmonic emission. The PV plants production cycle starts at 06:20 and end at 19:00 during the selected day presented in Figure 5-6. 


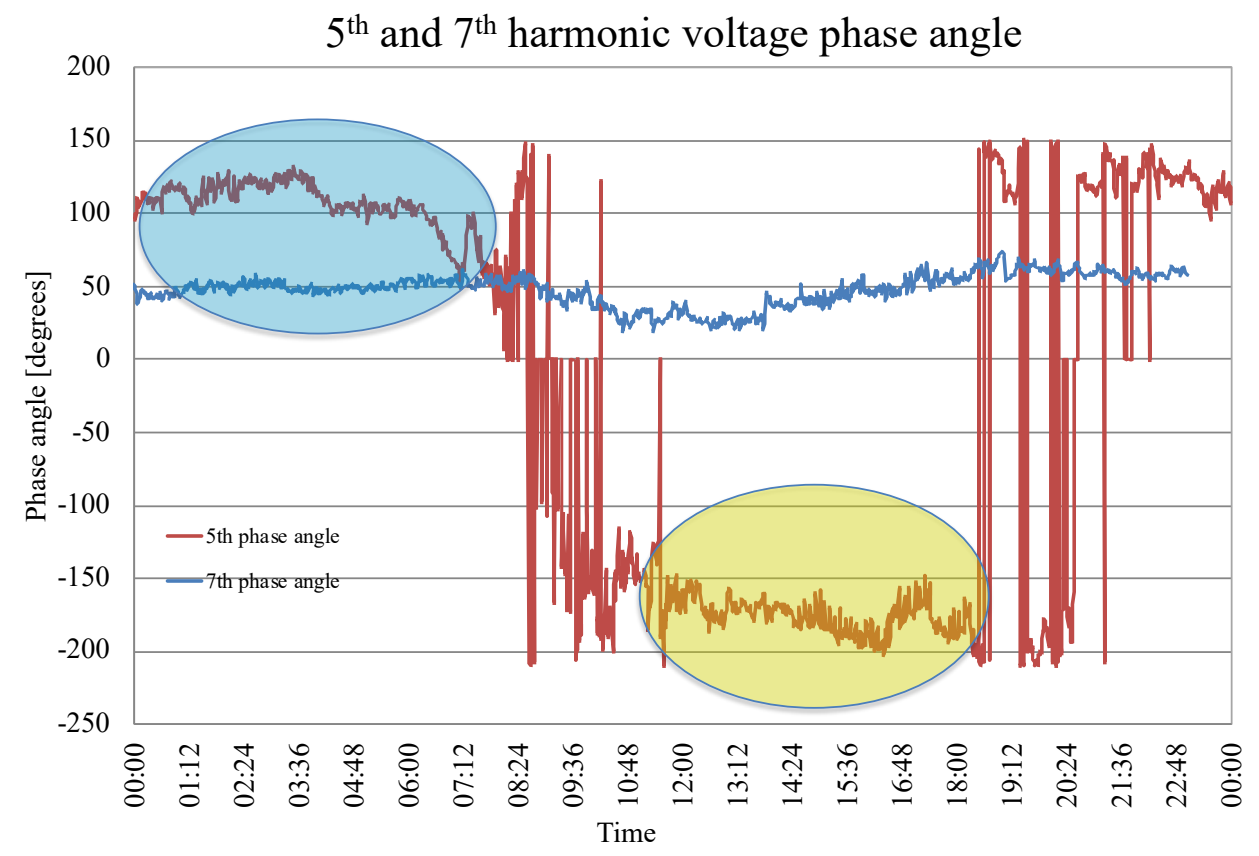

Figure 5-6. $5^{\text {th }}$ and $7^{\text {th }}$ harmonic voltage phase angle

Two distinct modes of operation for the $5^{\text {th }}$ harmonic voltage is highlighted in Figure 5-6. By using the harmonic phase angle, the condition when the PV plant is producing energy and when not, is now evident. When the PV plant is not generating, the $5^{\text {th }}$ harmonic voltage phase angle remains between $100^{\circ}$ and $130^{\circ}$. When the PV plant injects energy, the $5^{\text {th }}$ harmonic voltage phase angle remains mostly between $-150^{\circ}$ and $-200^{\circ}$. Some transient conditions occur during periods of low production.

With the knowledge that the PV plant [44]-[46] do produce (inject) a $5^{\text {th }}$ harmonic current and using the voltage phase angle information in Figure 5-6, it can be concluded that the concept of "emission" by the PV plant can only be assigned to the range of harmonic current phasors during the period when the PV plant is in generation mode.

By comparison of the $5^{\text {th }}$ harmonic emission phenomena as discussed above to the $7^{\text {th }}$ harmonic voltage phase angle, it is clear that in remaining around $50^{\circ}$ the $7^{\text {th }}$ harmonic current do not noticeably affect the $7^{\text {th }}$ harmonic voltage phasor. For this case, using the aggregated harmonic phase angle alone do not contribute to a better understanding of harmonic emission at this $7^{\text {th }}$ harmonic order.

\subsubsection{Polar plot analysis}

A polar plot is a visual aid to display both the magnitude and the phase angle of a phasor as a single point on a chart. The polar plot format was used in [73] to display aggregated harmonic distortion data in order to analyse the behaviour of the harmonic source under investigation. In this section the polar plot is combined with a heat map to display the concentration of data points on the polar plot. The higher the concentration of phasor points on top of each other the redder the heat plot will be. Isolated points will appear in blue due to the low concentration of phasor points.

All measured harmonic datapoints are taken into account in this section. Some of the measurements for $5^{\text {th }}$ and $7^{\text {th }}$ harmonic current phasors are during conditions not regarded as emission but included as this represent monitoring over a full period. 
Figure 5-7 and Figure 5-8 shows the $5^{\text {th }}$ and $7^{\text {th }}$ harmonic voltage phasor plots for the measurement point indicated in Figure 5-1.

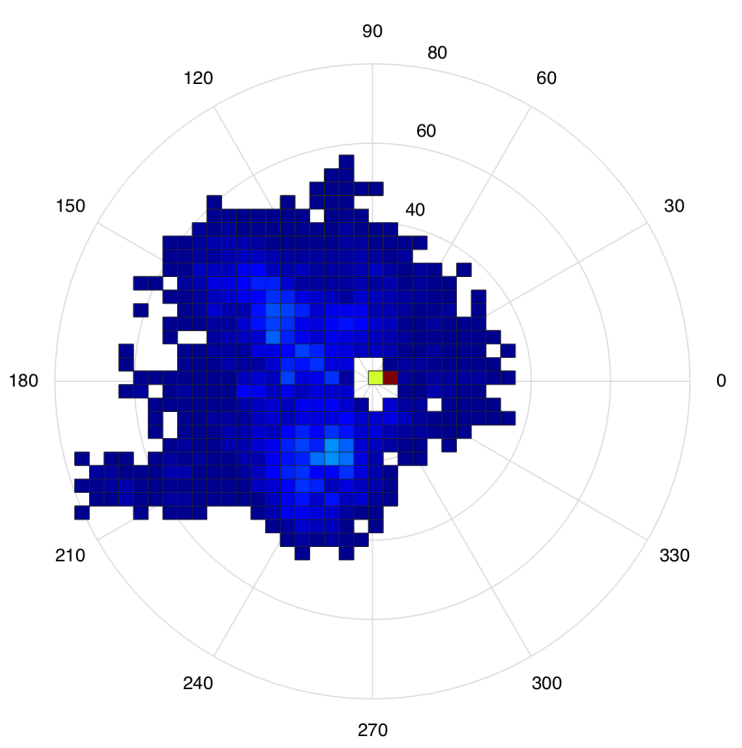

Figure 5-7. $5^{\text {th }}$ harmonic voltage polar heat plot

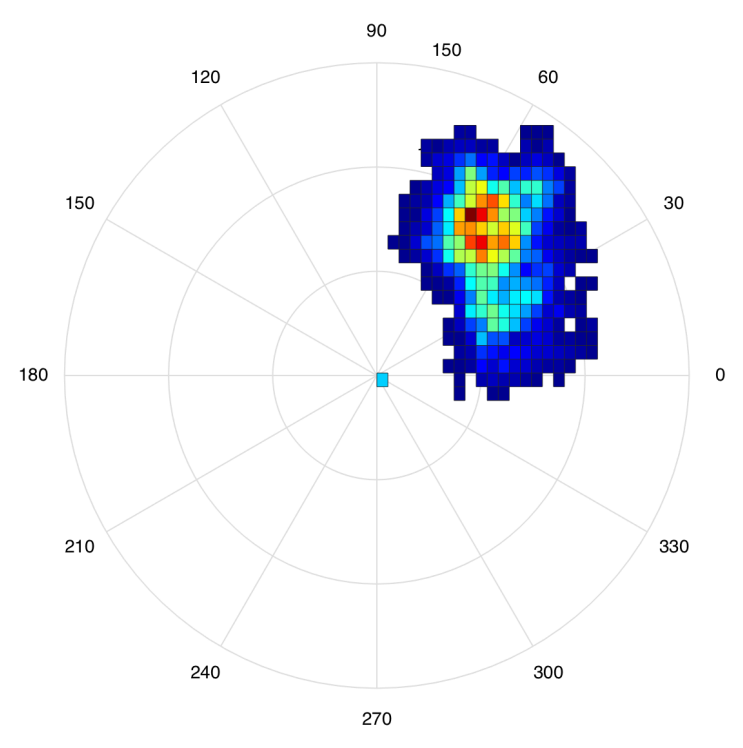

Figure 5-8. $7^{\text {th }}$ harmonic voltage polar heat plot

Using the field data set at hand, the goal is demonstrating the possible contradictions in harmonic emission assessment when not considering the phase angles of harmonics. First, the $5^{\text {th }}$ and $7^{\text {th }}$ voltage harmonics are evaluated.

Polar heat plot of the aggregated $5^{\text {th }}$ harmonic voltage phasors results in datapoints to be located in all four quadrants as shown in Figure 5-7 with most located in the $2^{\text {nd }}$ and $3^{\text {rd }}$ quadrant. Figure 5-6 showed that the $5^{\text {th }}$ harmonic phase angle changed from approximately $100^{\circ}$ when the PV plant is not in operation to approximately $-160^{\circ}$ when the PV plant is in operation. Fluctuations in the phase angle is also visible in Figure 5-6 as the power point tracking unit of the PV plant is seeking the maximum power output for the solar irradiance present when the sun rises and when the sun sets.

Comparison of the difference in dispersion between datapoints for the $5^{\text {th }}$ and $7^{\text {th }}$ voltage harmonic phasor illustrates the value of using a polar plot with a heat plot superimposed. In section 5.3.1 it was concluded that the $7^{\text {th }}$ voltage harmonic is largely dominated by the supply network as no clear evidence could be found that the $7^{\text {th }}$ harmonic current from the PV plant increase the $7^{\text {th }}$ voltage harmonic. In fact, limited change in the $7^{\text {th }}$ voltage harmonic was observed when comparing daylight hours (emission to expected) and nighttime hours.

Illustrated by Figure 5-8 is that most of the $7^{\text {th }}$ voltage harmonic phasors (aggregated) are located within close proximity, almost all in the first quadrant between $30^{\circ}$ and $90^{\circ}$. Emission at the $7^{\text {th }}$ voltage harmonic using this approach indicate that no contribution to the $7^{\text {th }}$ voltage harmonic from the $7^{\text {th }}$ harmonic current flowing from the PV plant to the supply network, could be found.

Practically it means that the PV plant has no effect on the $7^{\text {th }}$ voltage harmonic. Using field data, the normal application of harmonic emission methodologies (i.e. the CIGRE/CIRED C4.109 scatter plot method) requires comparing the calculated $7^{\text {th }}$ harmonic voltage rms values (obtained from the system impedance value and the measured harmonic current rms values), to the contracted values. This is now not needed as the $7^{\text {th }}$ voltage harmonic is not affected at all. The possibility of the PV plant found non-compliant by the "normal" approach is now eliminated, an important validation to the rationale of the research reported in this thesis. 
Secondly, analysis of polar heat plots for the $5^{\text {th }}$ and $7^{\text {th }}$ harmonic current phasors shown in Figure 5-9 and Figure 5-10 support the above conclusions on the corresponding voltage harmonics.

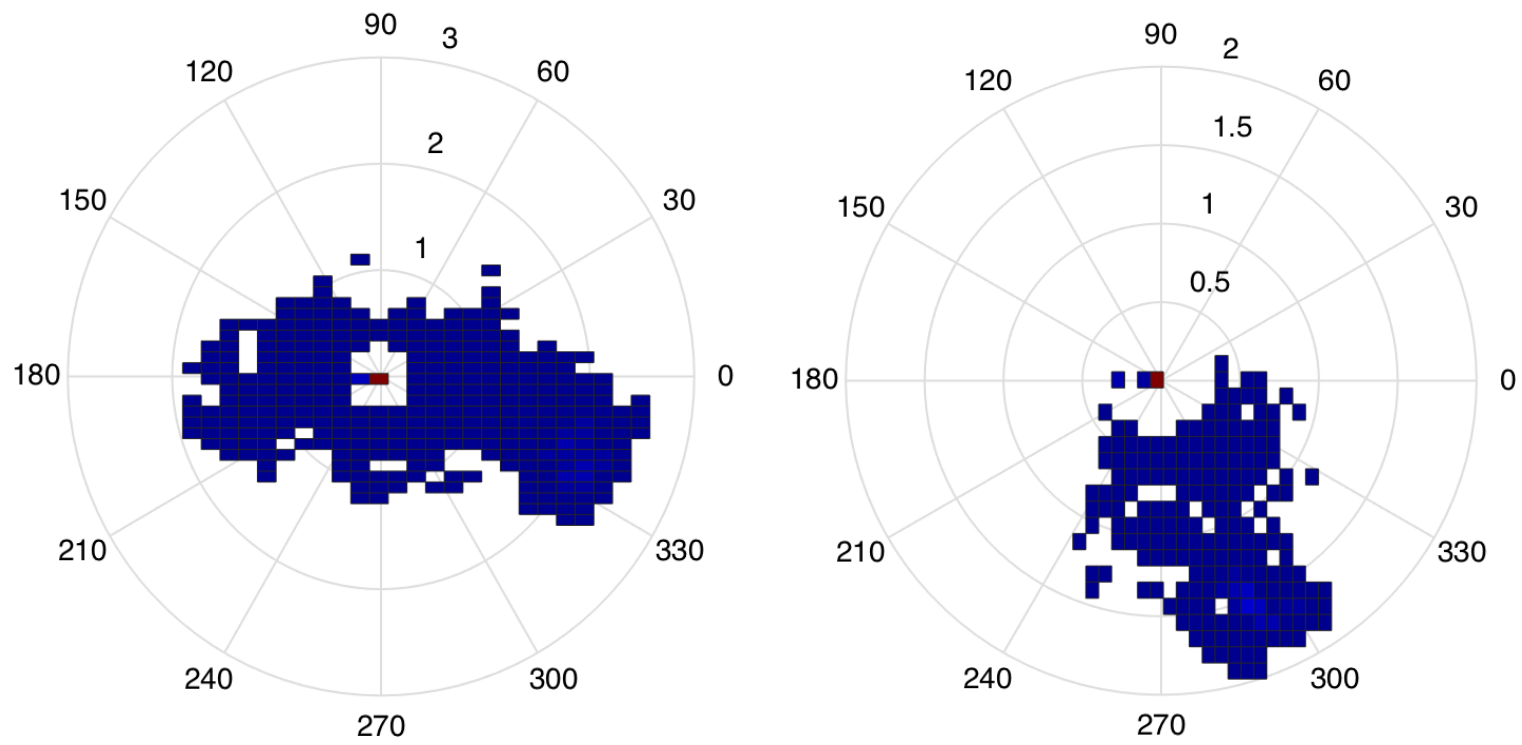

Figure 5-9. $5^{\text {th }}$ harmonic current polar heat plot [A] Figure 5-10. $7^{\text {th }}$ harmonic current polar heat plot [A]

$5^{\text {th }}$ harmonic current phasors are located in all four quadrants although horizontally dispersed. The location of the polar points to the left and right hand side of the vertical axis, indicate a directional $\left(180^{\circ}\right)$ change in the harmonic current phasor, confirming the observation obtained from the $5^{\text {th }}$ voltage harmonic, namely that the PV plant both absorb and emit harmonic emission.

The $7^{\text {th }}$ harmonic current phasors remain mostly in the fourth quadrant, with a few samples found in the third quadrant suggesting that a single harmonic source is responsible for this harmonic order. It was shown in the previous section that the $7^{\text {th }}$ harmonic current does not influence the $7^{\text {th }}$ harmonic voltage and it may be deduced that the $7^{\text {th }}$ harmonic current cannot be considered as emission.

The levels of prevalence (using (37))) is listed in Table 5-1. All datapoints were used, i.e. both conditions of harmonic emission and absorption exist in the aggregated harmonic phasors.

Important features when applying the principle of testing for prevalence follows from this:

1. From the literature [73], [80] the levels of prevalence in the $5^{\text {th }}$ harmonic voltage phasor is too low (0.61) and should not be used. The authors of [73], [80] based their recommendation of a prevalence level of 0.8 on a subjective view to what measure of phasor stability can be regarded as "prevalent". Their focus was in LV networks on the assessment of distorting loads and sources. In this research, their principle is extended to MV networks with the goal of improving harmonic emission assessment and the relative "low" level of prevalence are because the $5^{\text {th }}$ voltage harmonics are not all emission, they are also absorbed by the PV plant, upholding the principle that not all datapoints during field studies are useful for grid code compliance studies on harmonic emission. The level of prevalence in the $5^{\text {th }}$ harmonic current phasor at 0.84 is regarded as a harmonic phasor that is sufficiently stable during the aggregation periods applied to the $10 / 12$ cycle block harmonic phasors.

2. The $7^{\text {th }}$ harmonic voltage and current phasors are both significantly stable at a prevalence factor of 0.96 . This confirms the above conclusions that the $7^{\text {th }}$ voltage and current 
harmonic are mostly from one source during all of the time ( $7^{\text {th }}$ harmonic current phasor remains mostly in one direction).

Table 5-1. $5^{\text {th }}$ and $7^{\text {th }}$ harmonic phasors: level of prevalence

\begin{tabular}{|c|c|c|}
\hline & $5^{\text {th }}$ harmonic & $7^{\text {th }}$ harmonic \\
\hline Voltage phasor & 0.61 & 0.96 \\
\hline Current phasor & 0.84 & 0.96 \\
\hline
\end{tabular}

Power production of a PV plant is variable and consequently, harmonic emission is variable as well. In addition, changes to the upstream network configuration due to outages, equipment failure, and switching of reactive compensation devices will all change the harmonic impedance profile of the supply network, leading to additional factors influencing harmonic emission.

By distinguishing between voltage and current harmonics being measured during the day and night, the prevalence levels for the $5^{\text {th }}$ harmonic voltage is listed in Table 5-2. No $5^{\text {th }}$ harmonic prevalence level can be calculated for nighttime measurements as the harmonic current was zero amps during this period.

Table 5-2. Levels of prevalence for the day and nighttime for the $5^{\text {th }}$ harmonic

\begin{tabular}{|c|c|c|}
\hline $5^{\text {th }}$ harmonic & Day & Night \\
\hline Voltage phasor & 0.75 & 0.98 \\
\hline Current phasor & 0.89 & N/A \\
\hline
\end{tabular}

From Table 5-2 it is evident that when no harmonic emission from the PV plant exists, the $5^{\text {th }}$ harmonic voltage level of prevalence is near unity as expected. During the day, this level of prevalence is at 0.75 as both the network and the load (PV plant) contribute to the $5^{\text {th }}$ harmonic voltage in varying proportions. Important to note is that the $5^{\text {th }}$ harmonic current level of prevalence is not unity during the day. This would have been the case if all $5^{\text {th }}$ harmonic current was flowing from the PV plant to the supply network or vice versa, but as noted in Figure 5-4, during periods of low production the PV plant can also sink/absorb $5^{\text {th }}$ harmonic current.

\subsection{How much of harmonic emission is contributed by the PV plant?}

In section 4.3.1 a discriminative approach to harmonic emission was proposed using synchronised multiple-point measurements. By the analysis of network coherent data that reflect all points of interest to the investigation, it should be possible to better understand how different sources of harmonics contribute to harmonic emission.

An alternative approach to the identification of those harmonic currents regarded as emission, is presented next.

\subsubsection{Which values can be considered as harmonic emission from the PV plant?}

It was shown, using Figure 2-14, that from a utility point of view, harmonic emission realise when the harmonic voltage in their network is increased due to the integration of a new load, e.g. a PV plant. A Thévenin equivalent impedance $\left(\boldsymbol{Z}_{\mathrm{h}}\right)$ exist between the background harmonic voltage $\boldsymbol{E}_{h 0}$ and the PoC where the harmonic voltage $\boldsymbol{V}_{\mathrm{h}}$ realise due to a harmonic current $\boldsymbol{I}_{\mathrm{h}}$ causing a harmonic voltage drop $\boldsymbol{E}_{\mathrm{hc}}$. It is this voltage drop $\boldsymbol{E}_{\mathrm{hc}}$ that is of concern to the electrical utility. From this principle, a "proportional harmonic voltage emission percentage" is calculated by (42).

$$
\text { Proportional voltage emission }=\frac{V_{h}-E_{h 0}}{E_{h c}} * 100
$$


By using the same data set analysed in section 5.3, the results of equation(42) are shown in Figure 5-11. The top chart presents rms values for both the $5^{\text {th }}$ harmonic voltage $V_{5}$ and the background (network) $5^{\text {th }}$ harmonic voltage $E_{50}$. From the visual inspection, little difference is visible. With no difference at all, no harmonic emission exist as $V_{h}$ and $E_{h c}$ is equal (and $V_{h}$ is the result of only $\left.E_{\mathrm{hc}}\right)$. Such a scenario can arise when the PV plant is injecting a purely sinusoidal current into the supply network, resulting in no harmonic voltage drops across the Thévenin equivalent impedance. Some difference is noted in Figure 5-11 and is the goal of this section: to qualify and quantify the "proportional harmonic voltage emission" as a percentage, contributed by the PV plant.

The voltage emission $E_{h 5}$ shown in the middle chart of Figure 5-11 represent the absolute voltage harmonic emission calculated by (19). How much of $E_{h 5}$ is contributed by the PV plant can be found by application of (42) and shown in the bottom chart of Figure 5-11, regarded as the "proportional harmonic voltage emission".

Not all of the "proportional harmonic voltage emission" can be attributed to the PV plant. This principle was extensively discussed in the preceding chapters as the justification for the research reported in this document. A view that harmonic emission is realised when $\left|\boldsymbol{V}_{h}\right|>\left|\boldsymbol{E}_{h 0}\right|$ is generally agreed by electrical engineers.

By considering the above principle per harmonic order, when proportional harmonic voltage emission is negative, the PV plant is absorbing a harmonic current, reducing the voltage waveform distortion. Not all harmonic current phasors measured in a real network can be subjected to grid code compliance assessment studies (as stated previously).

If the proportional voltage emission is positive, then the PV plant is emitting harmonic emission into the network.
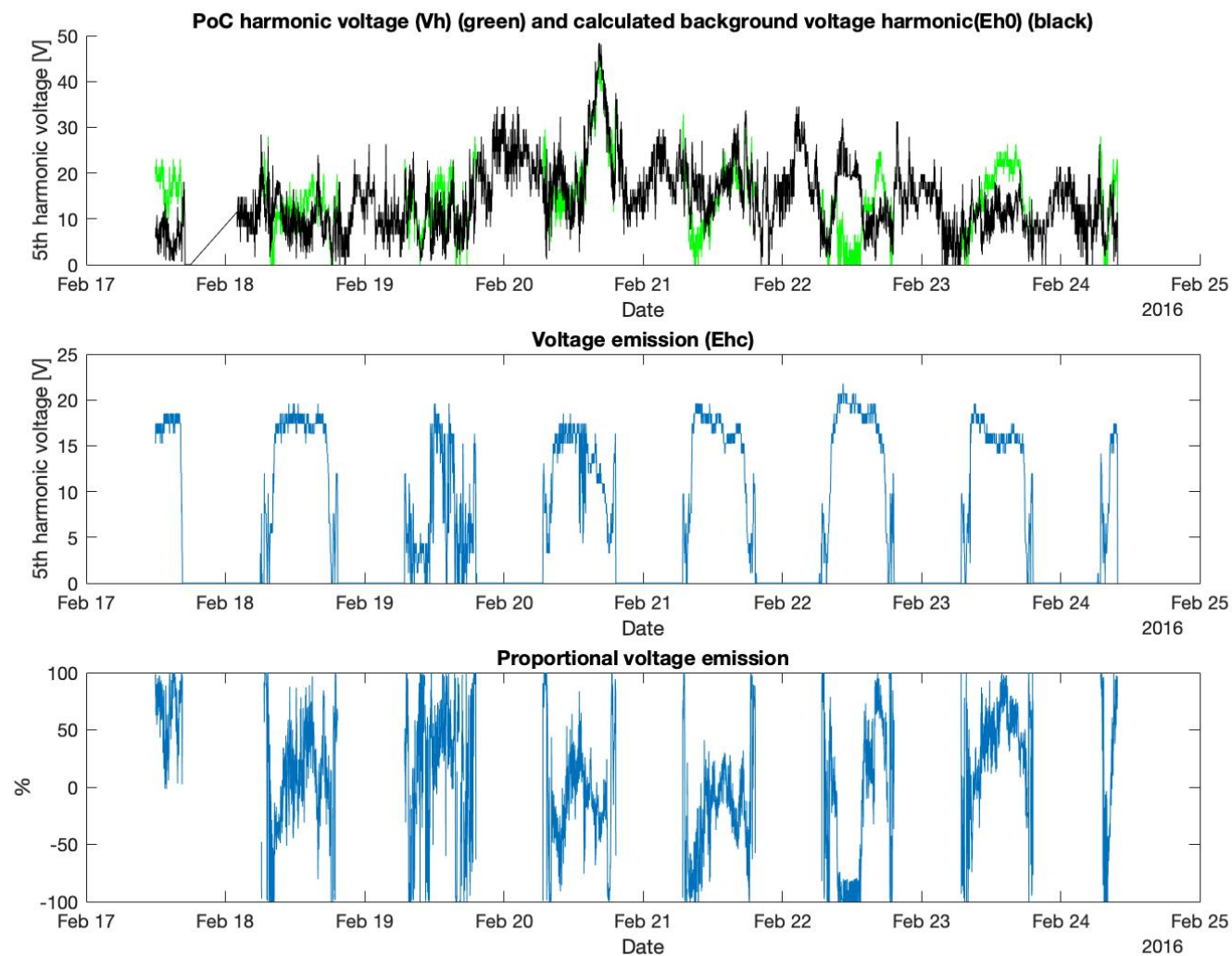

Figure 5-11. $5^{\text {th }}$ harmonic emission analysis from PV plant 
Figure 5-11 is yet another validation that the due to the non-linear interaction between a harmonic source and the supply network, a PV plant can sometimes improve harmonic distortion to later on deteriorate harmonic distortion. Even during the day, cloud cover and changes to the upstream network will influence harmonic emission of a PV plant.

- Harmonic emission assessment is not a singular exercise.

- Continuous monitoring of harmonic emission is needed (per harmonic order) to assess compliance to contractual agreements between the electrical utility and the grid user (PV plant owner).

Results obtained by analysis of $7^{\text {th }}$ harmonic emission is shown in Figure 5-12:

- The PV plant is absorbing $7^{\text {th }}$ harmonic distortion for most of the time when the plant is in operation.

This result confirms the conclusion made in section 5.3.3 that the $7^{\text {th }}$ harmonic current from the PV plant cannot be considered as emission and that it improves the network's $7^{\text {th }}$ harmonic voltage.
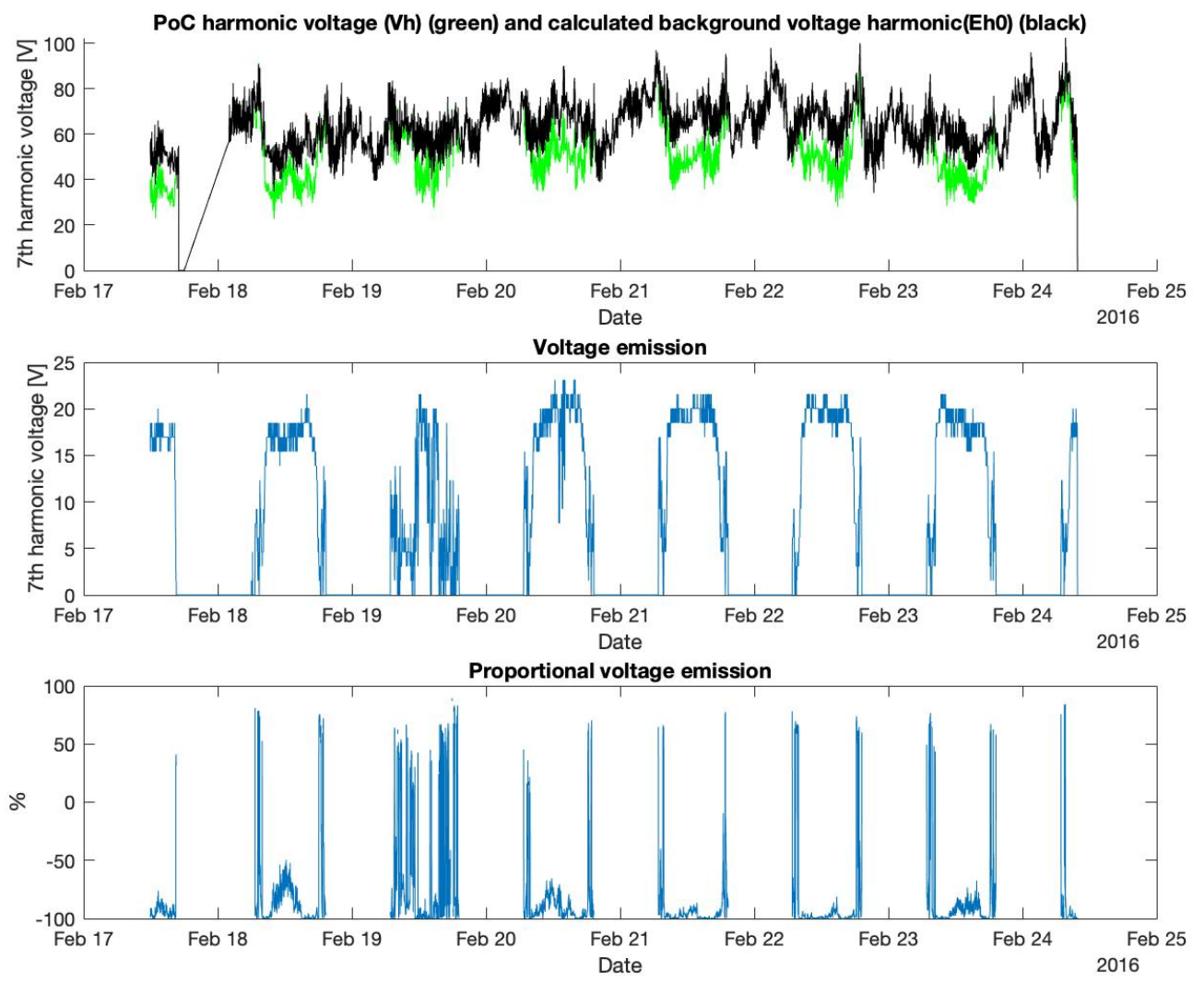

Figure 5-12. $7^{\text {th }}$ harmonic emission analysis from PV plant

\subsection{Field evaluation: range of secondary cancellation of harmonic phase angles}

The principle of identifying the range of phase angles of a specific harmonic phasor that reduce the voltage harmonic emission component $E_{h c}$ was presented in section 2.5.8. Referred to as the Range of Secondary Cancellation (RSC), it is another approach to improve harmonic emission assessment.

This concept has only been presented in theory [73] with no field application to be found in literature. In this thesis the concept is used to support the harmonic emission assessment done for the PV plant discussed in the previous sections. 
The RSC technique is site specific as the harmonic impedance, the harmonic voltage and current phasor measurements are used to calculate the maximum and minimum angles where secondary cancellation takes place. A detailed evaluation of the theory is discussed in section 2.5.8, and applied in section 3.7.

Field data of the PV plant under investigation in the previous sections are subjected to the RSC principle, and then secondly, to an industrial load that use harmonic filters to contain harmonic emission at a PoC to the supply network.

\subsubsection{Secondary cancellation of harmonic phase angles at a PV plant (RSC principle)}

The RSC principle is applied to discriminate between harmonic distortion being emitted from the PV plant and harmonic distortion being absorbed by the PV plant. Figure 5-9 has shown that the aggregated $5^{\text {th }}$ harmonic current phasors are located in all 4 quadrants. The aggregated $7^{\text {th }}$ harmonic current phasors are located only in one quadrant.

Table 5-3 lists the results obtained by application of (23). Background voltage harmonic distortion is regarded as the condition when the PV plant is not in operation (night time). The prevailing harmonic current phasor can only reflect the time when the PV plant produce current (daytime). Network impedance information was obtained from the utility.

Table 5-3. Calculated values and results for harmonic current RSC

\begin{tabular}{|c|c|c|c|c|}
\hline & \multicolumn{2}{|c|}{$5^{\text {th }}$ harmonic phasor } & \multicolumn{2}{|c|}{$7^{\text {th }}$ harmonic phasor } \\
\hline & Mag & angle $\left[{ }^{\circ}\right]$ & Mag & angle $\left[{ }^{\circ}\right]$ \\
\hline $\begin{array}{l}\text { Background voltage distortion } \\
\qquad \boldsymbol{U}_{p r v, h, M V}[\mathrm{~V}]\end{array}$ & 20.8 & 112.0 & 68.1 & 19.3 \\
\hline Prevailing current $\boldsymbol{I}_{p r v, h}[\mathrm{~A}]$ & 18 & -17.7 & 1.4 & -68.5 \\
\hline Network impedance $\boldsymbol{Z}_{h}(\Omega)$ & 8.4 & 87.5 & 11.9 & 87.9 \\
\hline $\begin{array}{l}\text { Range of secondary cancellation } \\
\varphi_{I, p r v(\max )}\left[^{\circ}\right]-\varphi_{I, p r v(\min )}\left[^{\circ}\right]\end{array}$ & & $93.3 ;-44.2$ & & $14.2 ;-151.4$ \\
\hline
\end{tabular}

Table 5-3 is visualised in Figure 5-13 and Figure 5-14.

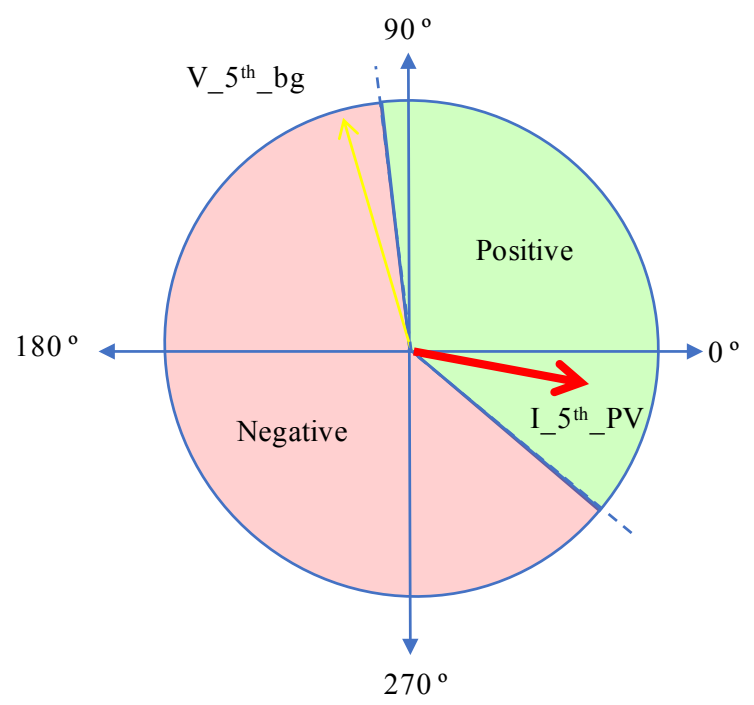

Figure 5-13. RSC for the $5^{\text {th }}$ harmonic current

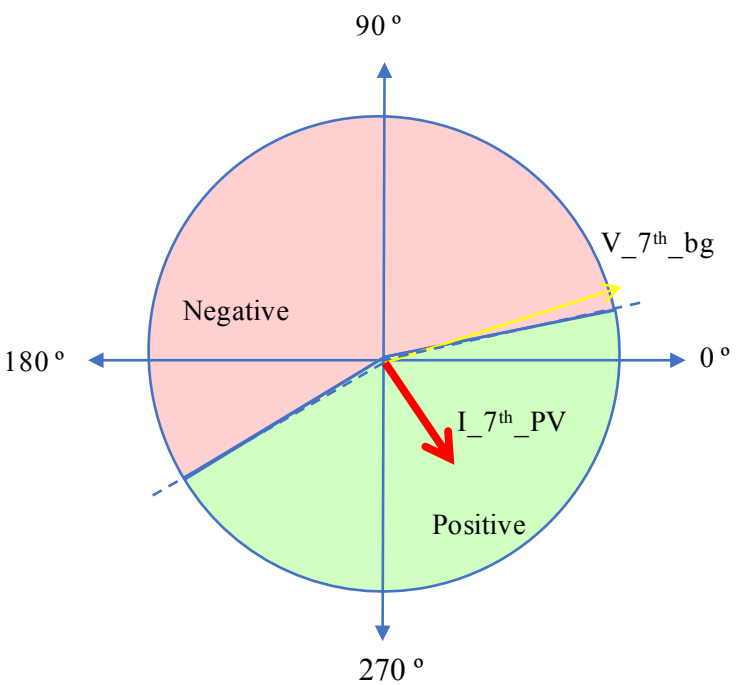

Figure 5-14. RSC for the $7^{\text {th }}$ harmonic current 
The prevailing harmonic current (red arrow in Figure 5-13 and Figure 5-14), representing the entire measurement dataset, for both the $5^{\text {th }}$ and the $7^{\text {th }}$ harmonic operates in the RSC positive area indicating that the harmonic current has a positive effect on the $5^{\text {th }}$ and $7^{\text {th }}$ voltage harmonic. $5^{\text {th }}$ harmonic prevailing current value does not operate in the middle of the green area but slightly to the one side in the fourth quadrant, this is due to some of the harmonic current values not being considered as emission, as confirmed by Figure 5-11. $7^{\text {th }}$ harmonic current operates in the middle of the positive range indicating that the PV plant has a positive impact on the harmonic voltage for the $7^{\text {th }}$ harmonic, as confirmed by Figure 5-12.

\subsubsection{Harmonic filter performance assessment}

To further affirm the RSC principles, the technique was applied to an industrial plant consisting of two DC arc furnaces supplied from a $33 \mathrm{kV}$ network as shown in Figure 5-15. The Distribution System Operator (DSO) allocated specific harmonic emission limits to this industrial load. For this industrial load to meet the requirements set out by the DSO, passive harmonic filters were installed to mitigate the harmonic emissions to below the DSO's limits. The harmonic filters are further coupled with a Thyristor Controlled Reactor (TCR).

Performance of harmonic filters are evaluated during commissioning of the units or when an operator suspects that there may be problem due to plant failures.

Passive harmonic filter performance is affected by the impedance in the supply network and in the components used in the filter design. How well a filter is performing over an extended period of time that includes possible network impedance changes, is mostly not known.

It is shown in this section by the evaluation of the range of phase angles at a specific harmonic order, the performance of the harmonic filter can be evaluated. A well-designed harmonic filter will contain voltage harmonic phasors at the PoC to below the DSO's allocated emission limits by sinking current harmonic phasors [101] through the filter.

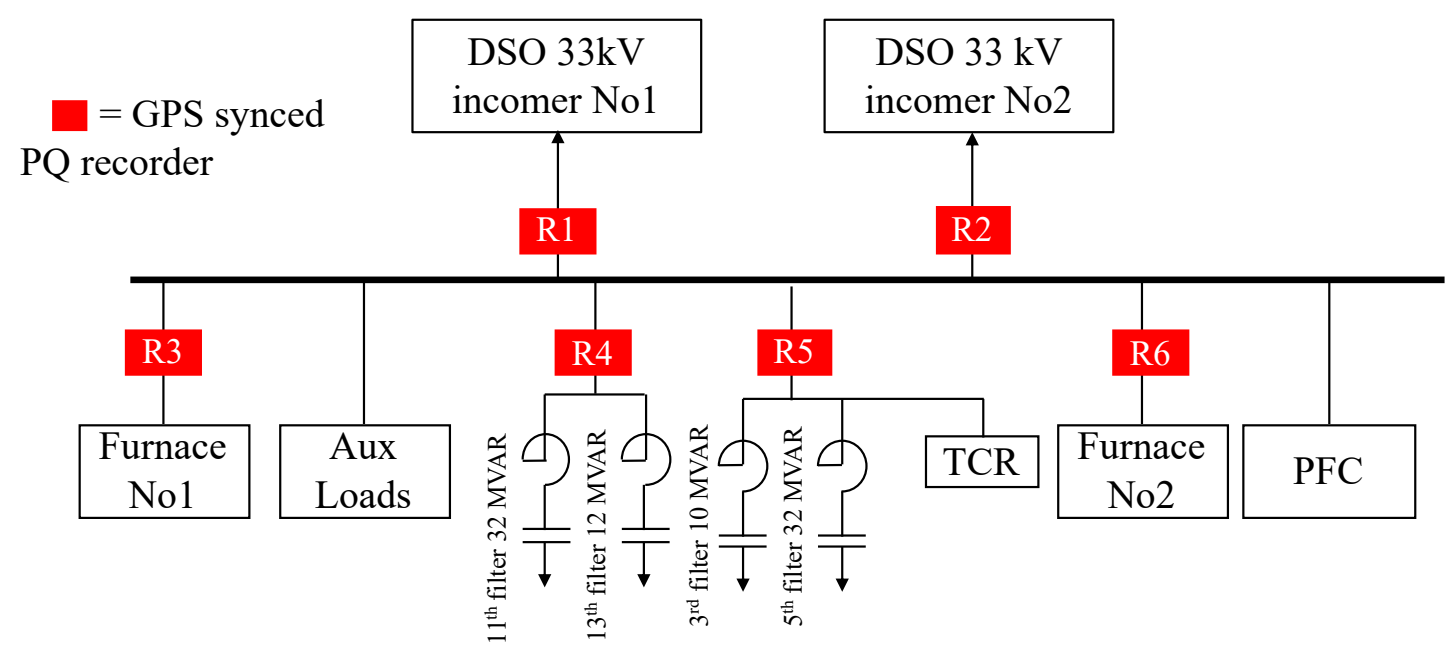

Figure 5-15. Industrial load network diagram

PQ recorders that are IEC 61000-4-30 class A compliant were installed for a period of over 60 days at the points indicated in Figure 5-15. For analysis and visualisation purposes only one day's data is presented. The selected day contains periods where the harmonic filters were out of service making this day suitable to apply the RSC technique. Figure 5-16 to Figure 5-19 shows the recorded data for the $3^{\text {rd }}, 5^{\text {th }}, 11^{\text {th }}$ and $13^{\text {th }}$ harmonics as these are the harmonic orders for which mitigation was installed. 


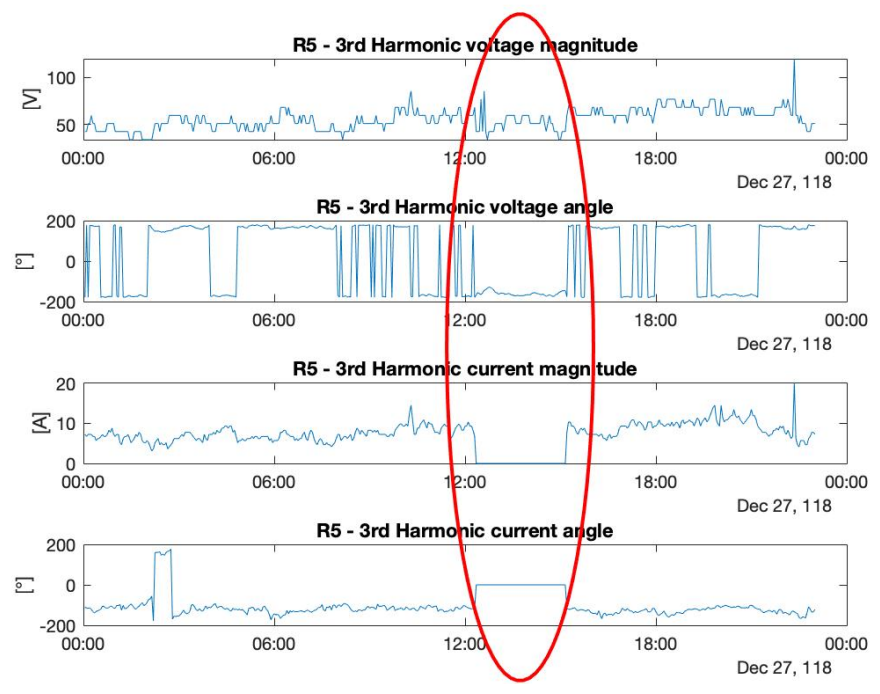

Figure 5-16. $3^{\text {rd }}$ harmonic voltage and current magnitude and phase angle plots
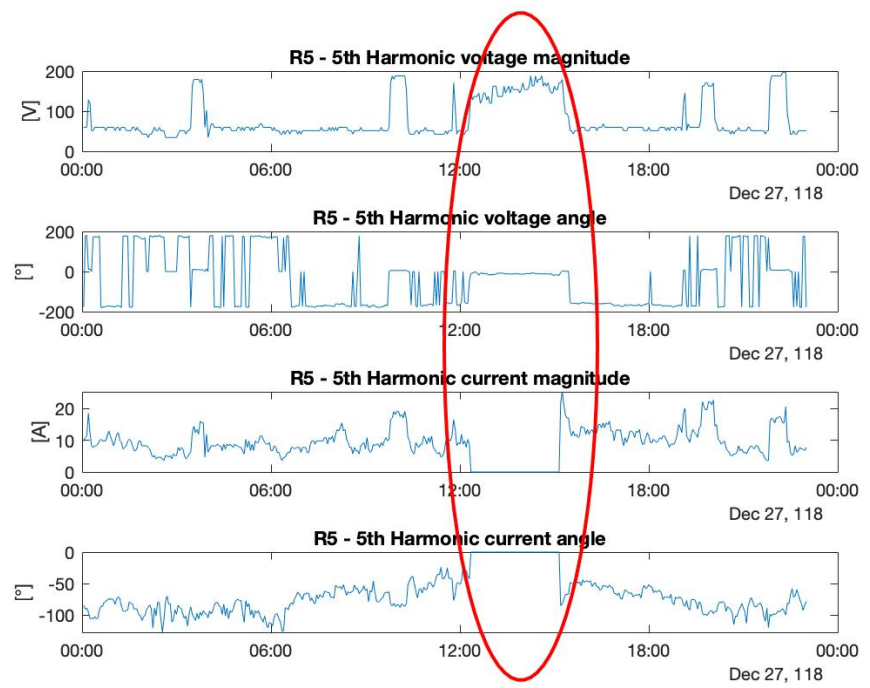

Figure 5-17. $5^{\text {th }}$ harmonic voltage and current magnitude and phase angle plots
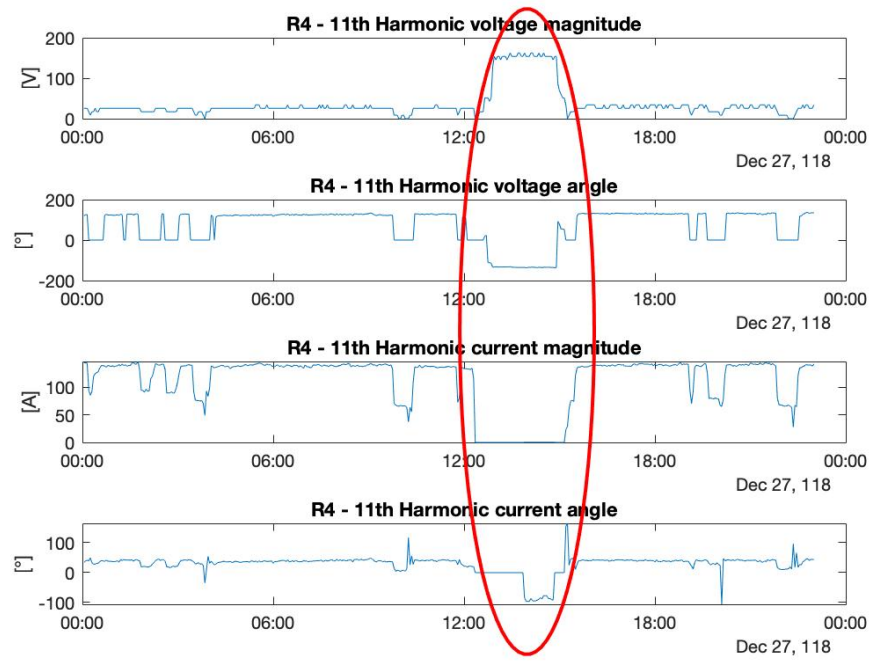

Figure 5-18. $11^{\text {th }}$ harmonic voltage and current magnitude and phase angle plots 


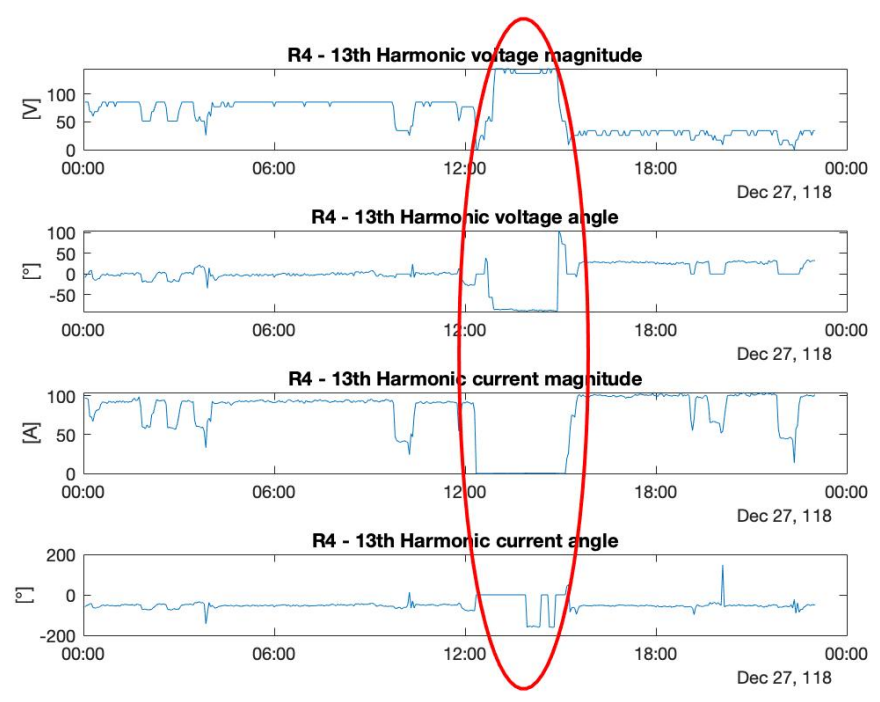

Figure 5-19. $13^{\text {th }}$ harmonic voltage and current magnitude and phase angle plots

Red ellipses in Figure 5-16 to Figure 5-19 highlight the time of day when the harmonic filters were out of service. Note that all of the filters were taken out of service at the same time.

Observe in Figure 5-16 that there is no change in the $3^{\text {rd }}$ harmonic voltage profile when the harmonic filter is switched out of service in comparison to when the harmonic filter is in service. This indicates that the $3^{\text {rd }}$ harmonic filter is not effective in reducing the harmonic voltage phasor.

In contrast significant harmonic voltage changes can be seen when the $5^{\text {th }}, 11^{\text {th }}$ and $13^{\text {th }}$ filter are switched out of service as highlighted by the red ellipses (Figure 5-17 to Figure 5-19), when compared to the filters being in service indicating that these harmonic orders are being mitigated.

The selected data displayed in Figure 5-17 to Figure 5-19 was applied to (23) to calculate the RSC values for the respective harmonic orders. Network harmonic impedance values to be used in (23) was obtained from the DSO. Table 5-4 list the values used, and the phase angle results for the $3^{\text {rd }}$ and $5^{\text {th }}$ harmonics.

Table 5-4. Calculated values and results for harmonic current RSC for the $3^{\text {rd }}$ and $5^{\text {th }}$ harmonic

\begin{tabular}{|c|c|c|c|c|}
\hline & \multicolumn{2}{|c|}{$3^{\text {rd }}$ harmonic } & \multicolumn{2}{|c|}{$5^{\text {th }}$ harmonic } \\
\hline & Mag & angle $\left[{ }^{\circ}\right]$ & Mag & angle [ $\%$ \\
\hline $\begin{array}{l}\text { Background voltage distortion } \\
\boldsymbol{U}_{p r v, h, M V}[\mathrm{~V}]\end{array}$ & 51.8 & 19.2 & 153.8 & -11.6 \\
\hline Prevailing current $\boldsymbol{I}_{p r v, h}[\mathrm{~A}]$ & 7.3 & 59.2 & 9.6 & -78.1 \\
\hline Network impedance $\boldsymbol{Z}_{h}(\Omega)$ & 4.0 & 71.5 & 4.8 & 84.6 \\
\hline $\begin{array}{c}\text { Max range of cancellation } \\
\varphi_{I, \operatorname{prv}(\max )}\left[^{\circ}\right]\end{array}$ & & 21.4 & & -14.9 \\
\hline $\begin{array}{c}\text { Min range of cancellation } \\
\varphi_{I, \operatorname{prv}(\min )}\left[{ }^{\circ}\right]\end{array}$ & & -125.9 & & -177.6 \\
\hline
\end{tabular}

Figure 5-20 and Figure 5-21 overlaps the visualised minimum and maximum phase angle ranges listed in Table 5-4 and a polar heat plot of the harmonic current data measured over 60 days for the $3^{\text {rd }}$ and $5^{\text {th }}$ harmonics. The green angular range indicates where harmonic current values will have a positive impact on the harmonic voltage, while the red angular range shows where the harmonic current values will have a negative effect on the harmonic voltage. 


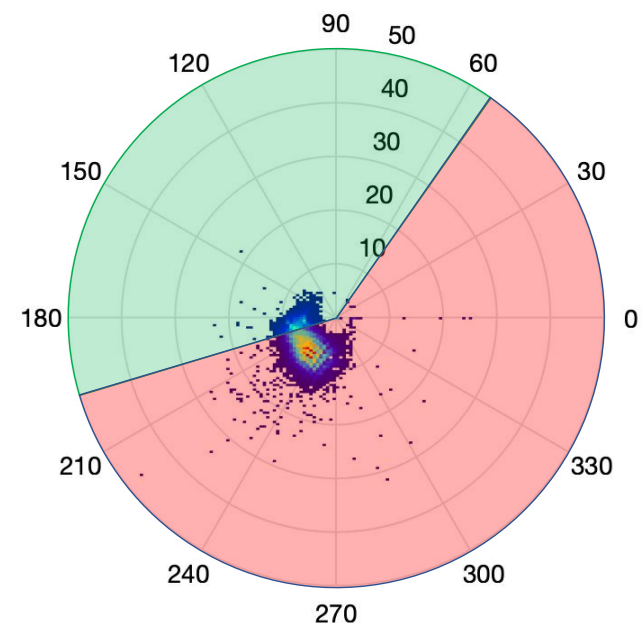

Figure 5-20. RSC visualization for the $3^{\text {rd }}$ harmonic current

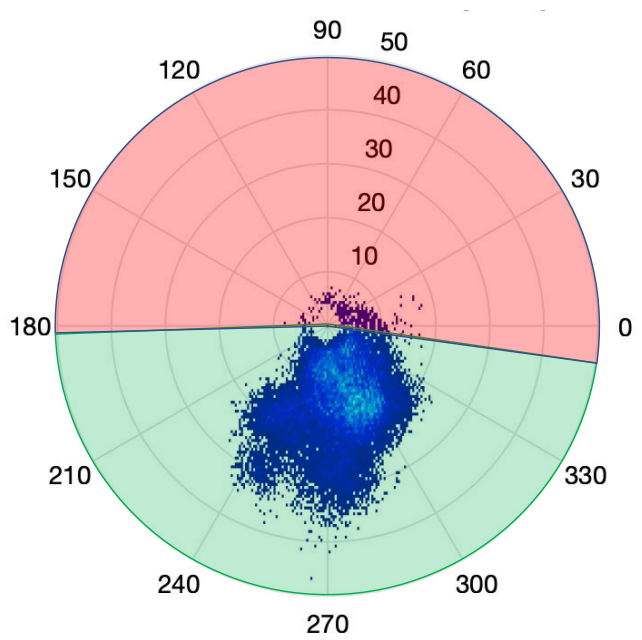

Figure 5-21. RSC visualization for the $5^{\text {th }}$ harmonic current

In Figure 5-20 a heat cloud is observed for the measured $3^{\text {rd }}$ harmonic current values, indicating a high concentration of data points in the third quadrant. These data points operate largely in the red angular range of the RSC technique meaning that the $3^{\text {rd }}$ harmonic current does not have a positive impact on the harmonic voltage. The $3^{\text {rd }}$ harmonic filter is not effective in containing the voltage harmonic phasor to below the DSO's limits, confirming the crude analysis done in Figure 5-16 when the filter is in and out of service.

No clear heat cloud is seen for the $5^{\text {th }}$ harmonic current values presented in Figure 5-21 operate in the green angular range and in the vicinity of the maximum secondary cancellation point located in the middle of the green area. It is confirmed through the RSC technique that the $5^{\text {th }}$ harmonic filter is effective in reducing the harmonic voltage phasors at the $\mathrm{PoC}$.

RSC values for the $11^{\text {th }}$ and $13^{\text {th }}$ harmonic could not be calculated. Prevailing phasors calculated result in $\left(I_{p r v} \cdot Z_{h}\right) /\left(2 \cdot U_{p r v}\right)$ from (23) being larger than 1 . With the harmonic measurements conducted correctly the only variable in question is the harmonic impedance values obtained from the utility. Due to the location of the measurements the harmonic impedance $Z_{h}$ should take into consideration the impact of the adjacent harmonic filters, resulting in a more accurate harmonic impedance value to be used for the RSC. Alternatively online measurements can be used to determine the harmonic impedance at the PoC [102].

\subsection{Conclusion}

Although a synthetic concept, the measurement of aggregated harmonic phasors was proven to be useful in the assessment of harmonic emission [103]. In literature, papers [99], [100], [104] applies the prevailing phase angle concept to low voltage and medium voltage networks. The main aim of these studies discussed in the papers was to determine the impact of electric vehicles and PV onto the harmonic performance of the network.

Grid Code compliance must be fair and practical in that a non-linear load such as a PV plant should not be penalised for aiding the DSO's network in reducing or improving the network harmonic voltage at a Point of Connection (PoC). A discriminative approach is thus needed to only account for the values which will have a negative impact on the PoC i.e. harmonic emission.

The CIGRE/CIRED C4.109 statistical approach as suggested by the South African Grid Code [1] is practical and simple when the data points align to the impedance loci, but when the data points 
are outside of the scope of interpretation then the assessment method delivers inconclusive results. Discrimination of the harmonic emission is thus not possible with this method when measured at a single point.

A field study is used as a comparison between the CIGRE/CIRED C4.109 method and the aggregation of harmonic phasors, known as the prevailing phase angle. The aim of which is to evaluate if the CIRED/CIGRE C4.109 method can be improved for a pragmatic engineer to use in the grid code compliance process, whilst still maintaining scientific integrity.

Interpretation of the CIGRE/CIRED C4.109 methods shows that the PV plant for the $5^{\text {th }}$ harmonic is the dominant emitter to the harmonic distortion at the PoC. In stark contrast, the prevailing phase angle method shows that the PV plant is actually both an emitter and an absorber of harmonic emission. All of the 10-minute rms values used in the CIGRE/CIRED C4.109 method can thus not be taken as being harmonic absorption only or emission only.

Polar plots of the $5^{\text {th }}$ harmonic current show the extent of emission and absorption in that the harmonic current phasors operate in all four quadrants.

Analysis on the PV plant using a new concept termed the Range of Secondary Cancellation (RSC) details the extent of how the PV plant is aiding the network, in that the PV plant is mostly absorbing the harmonic distortion from the upstream network. Affirmation of the RSC technique was done through further field testing at an industrial plant where the performance of harmonic filter banks were tracked. RSC can be used to track harmonic filter performance, but analysis can be hampered when inaccurate information is not obtained.

A further analysis on the PV plant using the relative emission calculation visually shows how much the PV plant is either absorbing or emitting harmonic distortion for the $5^{\text {th }}$ harmonic. This analysis further shows the dynamic behaviour of the $5^{\text {th }}$ harmonic over time and necessitates the need for continuous harmonic emission assessment.

The $7^{\text {th }}$ harmonic showed inconclusive results when the CIGRE/CIRED C4.109 method was applied. Without further guidelines on how to interpret the data using the CIGRE/CIRED C4.109 method, a dispute ensued between the PV plant owner (claiming his plant is not the source) and the Distribution System Operator (DSO) (claiming that the harmonic distortion is there due to the PV plant).

Application of the prevailing phase angle methodology shows that the PV plant is absorbing harmonic emission for most of the time when it is in operation. This absorption was proven by both the RSC technique and the relative emission concepts.

An interpretation of the rms time plots between the harmonic voltage and harmonic current does not show the reduction in the background harmonic distortion, highlighting the limitation of only using an rms value. It must, however, be noted that there are other large scale PV plants connected onto the DSO's $66 \mathrm{kV}$ network, which may have an influence on the background harmonic distortion.

In conclusion, the CIGRE/CIRED C4.109 method is limited in indicating which specific harmonic values are being emitted and which are being absorbed by a PV plant, due to the use of a 10-minute rms value only. Inclusion of aggregated harmonic phase angle data has shown how a PV plant can emit and absorb harmonic emissions, adding value to the fair and pragmatic assessment of harmonic emission in grid code compliance. 


\section{CHAPTER 6: Application of the prevailing phase phasor in a high voltage network}

\subsection{Introduction ${ }^{10}$}

In this chapter, the prevailing phase angle measurement method is applied to a high voltage network where multiple nonlinear loads are interconnected onto the same node. The aim of this application is to evaluate the impact of nonlinear loads on each other and to highlight the challenge in obtaining grid code compliance within this dynamic environment.

Focus is placed on two large scale PV plants and the impact of other nonlinear loads onto the assessment of harmonic emission as part of the grid code compliance process.

Available literature where the principle of prevailing phasors is discussed [91, 92, 96] were applied to low voltage networks. Due to the absence of large industrial loads in these networks, it can be argued that the harmonic phasors would be more stable under these conditions - and hence more applicable. It is, therefore, a useful contribution to evaluate prevailing phasors in high-voltage networks.

\subsection{Evaluation of harmonic emission at a distribution substation with multiple nonlinear loads connected to the same busbar}

Harmonic emission assessment for grid code compliance [1] is meant to take into account the harmonic emission that is only due to the RPP that is under evaluation. It has been shown in Chapter 4 that single-point harmonic measurements, with rms values only, cannot be used to accurately and fairly account for a plant's harmonic emission.

The value of aggregated harmonic phasor measurements has been proven in Chapter 5: at a medium voltage PV plant. Only the PV plant was connected to the PoC, which made for easy application of harmonic emission theory.

To further evaluate the application of the prevailing phase angle measurement method, a field study was conducted on a high voltage network with multiple non-linear loads connected to the same node/busbar. Multiple PQ instruments were installed to simultaneously measure the harmonic voltages and currents at a $132 \mathrm{kV}$ busbar where two large scale PV plants are connected as well as a traction network and other loads. Synchronisation between the PQ instruments was achieved by GPS timestamping as discussed before (section 4.3).

The same measurement system and uncertainty budget as discussed in section 4.4 will apply to the harmonic measurements shown in this chapter.

Figure 6-1 shows the network diagram with the PV plant under investigation and the location of the synchronised PQ recorders.

\footnotetext{
${ }^{10}$ Two peer-reviewed conference papers as well as a journal article were published on the research contained in this chapter [113]-[115].
} 


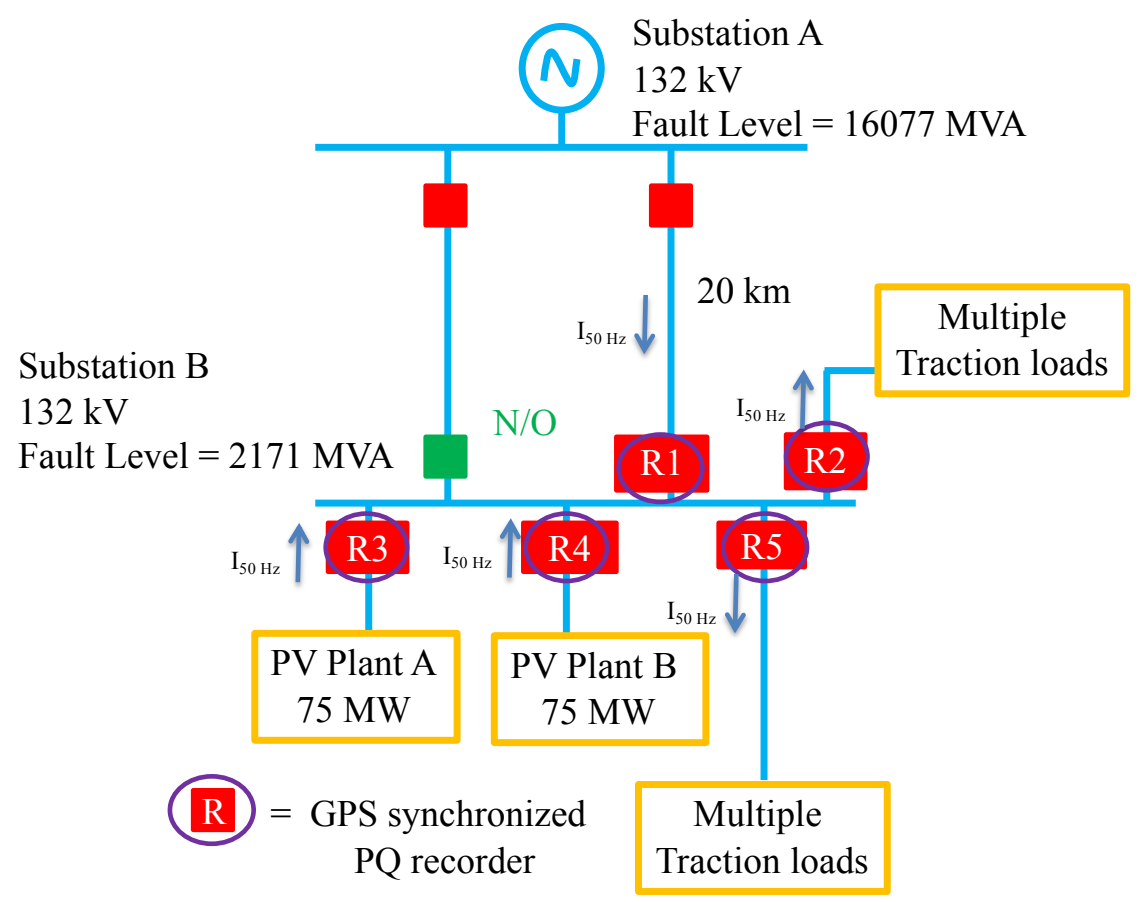

Figure 6-1. Network diagram and location of $P Q$ recorders

A total of five PQ recorders were installed as shown in Figure 6-1 all measuring harmonic voltage and current parameters. To limit the number of charts only the $5^{\text {th }}$ and $7^{\text {th }}$ harmonic will be presented. Each PQ recorder was compliant to IEC 61000-4-30 Ed. 3 [49] and had the prevailing phase angle algorithm implemented onto their firmware. Measurements were taken for a minimum of 7 days as per the grid code [1] requirements. The instrument time aggregation interval was selected to 1-min as per recommendation in [73].

The frequency sweep at substation B was obtained from the utility and shown in Figure 6-2.

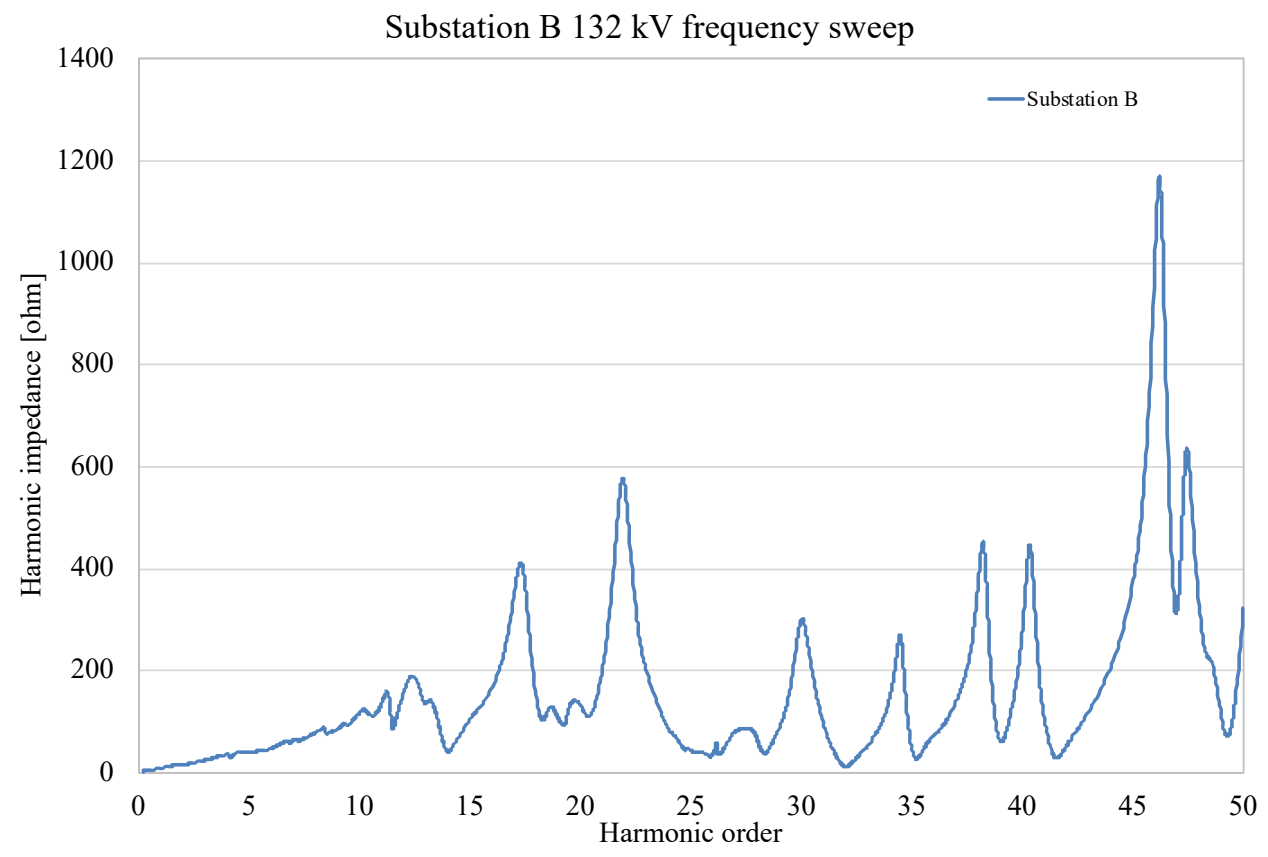

Figure 6-2. Frequency Sweep for the $132 \mathrm{kV}$ busbar at Substation $B$

Results from the measurement campaign using the prevailing angle theory are discussed next. 
Figure 6-3 and Figure 6-4 shows the $5^{\text {th }}$ and $7^{\text {th }}$ harmonic voltage polar heat plots respectively at substation B in Figure 6-1. Consider that the harmonic voltage at substation B is a summation of harmonic distortion from two large scale PV plants, multiple traction loads and some residential and light industrial loads.

Observe Figure 6-3 in that the $5^{\text {th }}$ harmonic voltage operates in quadrant 1 and 2 with a heat cloud in the second quadrant, indicating that most of the harmonic voltage phasors operate in the second quadrant. It is not clear as to which harmonic source can be considered to be the dominant contributor to the $5^{\text {th }}$ harmonic voltage distortion. The heat cloud suggests that there may be a dominant harmonic current source within the network however the influence of the low $5^{\text {th }}$ harmonic impedance $(38.81 \Omega)$ may result in a small $5^{\text {th }}$ harmonic voltage $(19)$ hence the heat cloud operating mainly in second quadrant (i.e. the upstream network is very stiff).

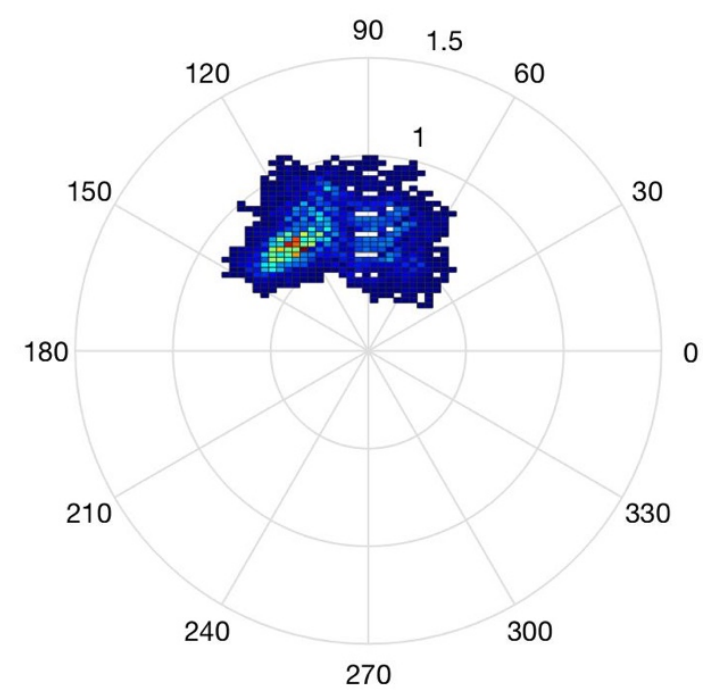

Figure 6-3. $5^{\text {th }}$ harmonic voltage polar heat plot at Substation $B[\mathrm{kV}]$

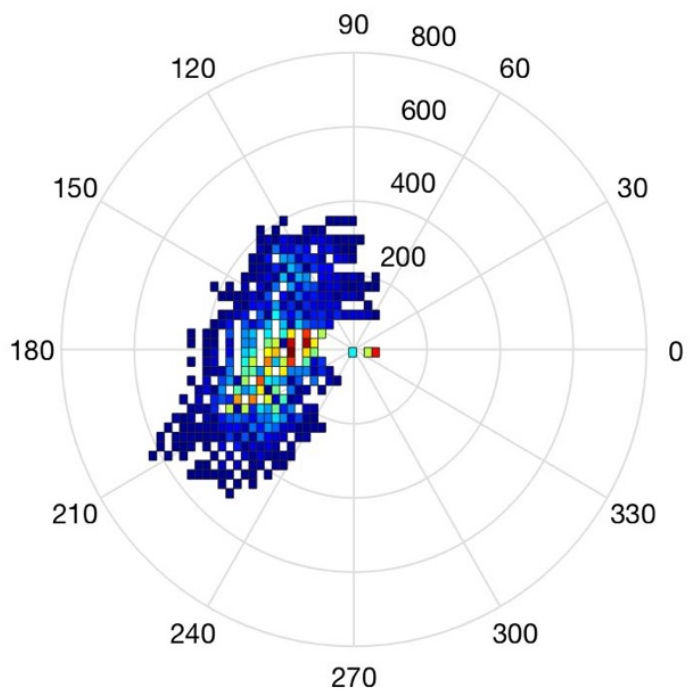

Figure 6-4. $7^{\text {th }}$ harmonic voltage polar heat plot at Substation $B[V]$

The $7^{\text {th }}$ harmonic voltage polar plot in Figure 6-4 shows that the harmonic distortion operates mainly in the second and third quadrant with a heat cloud between $180^{\circ}$ and between $210^{\circ}$.

Figure 6-5 to Figure 6-14 shows the harmonic current polar plots at R1 to R5 for the $5^{\text {th }}$ and the $7^{\text {th }}$ harmonics.

Heat plots cannot be drawn for Figure 6-7, Figure 6-8 and Figure 6-12 due to the low resolution of measurements resulting from the PQ recorder, which makes use of a 16 bit analogue to digital converter and due to the low harmonic current values measured. Normal polar plots are presented for the above mentioned figures. 


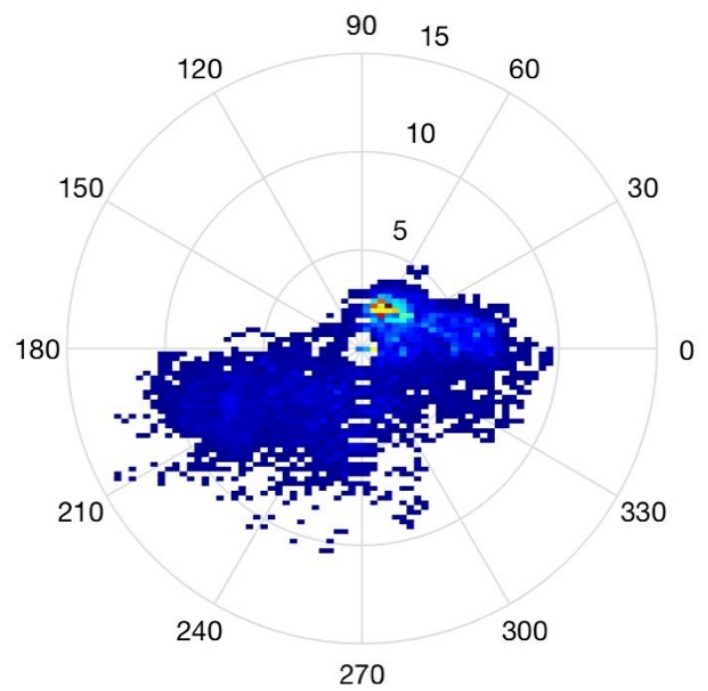

Figure 6-5. $5^{\text {th }}$ harmonic current polar heat plot at $R 1$ [A]

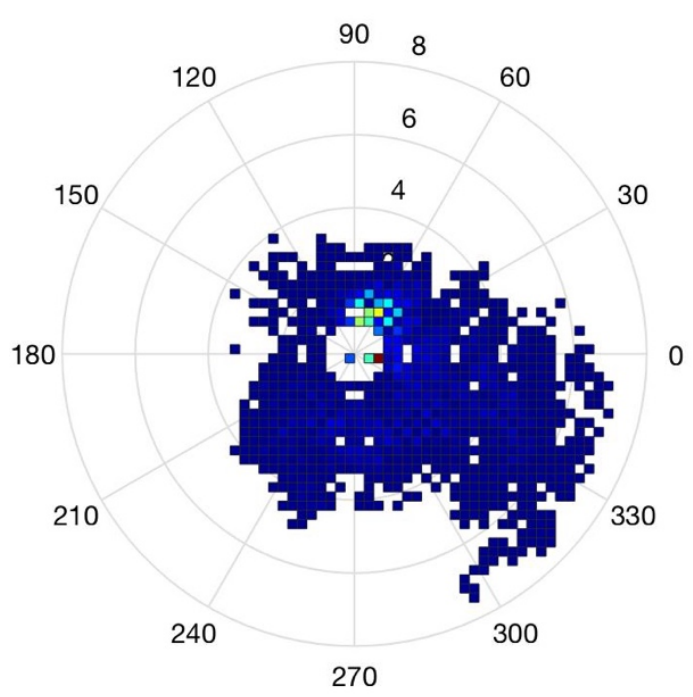

Figure 6-6. $7^{\text {th }}$ harmonic current polar heat plot at RI [A]

Harmonic phasor measurements at R1 shown in Figure 6-5 and Figure 6-6 the summated harmonic currents flowing from all of the harmonic sources connected to the $132 \mathrm{kV}$ busbar at Substation $\mathrm{B}$, as harmonics tend to flow upstream to the source [55]. Observe that the harmonic phasors for both the $5^{\text {th }}$ and the $7^{\text {th }}$ harmonic currents operate in all four quadrants. In section 3.4 simulations showed the influence of harmonic active power load flow between loads and the net effect that flows into the upstream network [16]. With the $5^{\text {th }}$ and $7^{\text {th }}$ harmonic current aggregated phasors operating in various quadrants, it is testament to the harmonic distortion interaction that is taking place between the non-linear loads as well as the upstream network, which confirm the simulations in section 3.4.

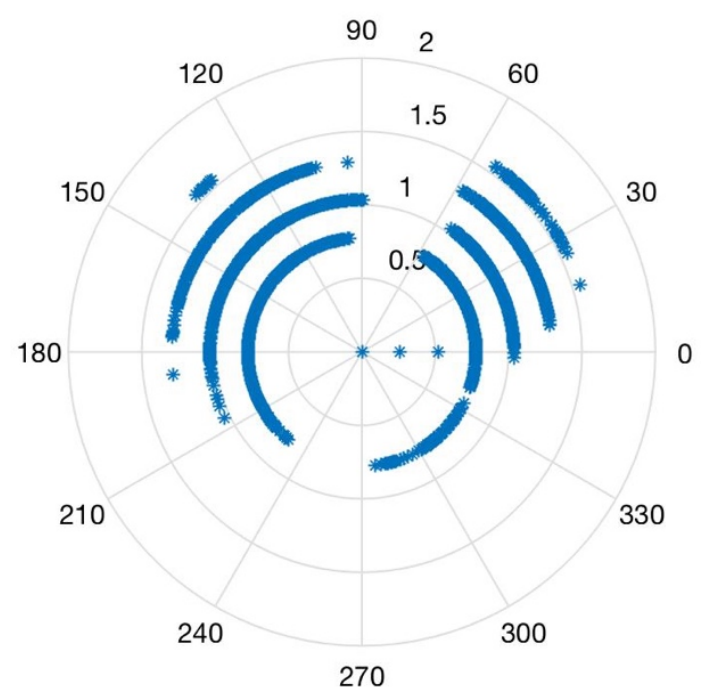

Figure 6-7. $5^{\text {th }}$ harmonic current polar plot at $R 2$ [A]

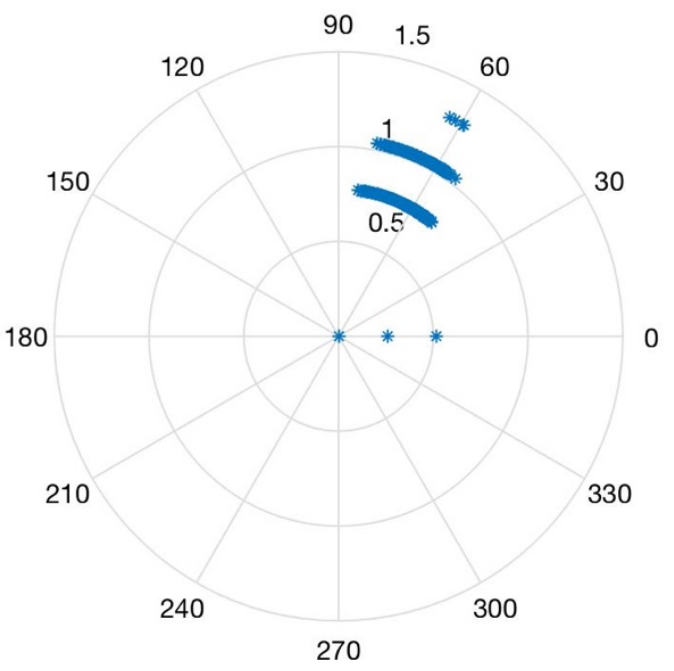

Figure 6-8. $7^{\text {th }}$ harmonic current polar plot at R2 [A] 

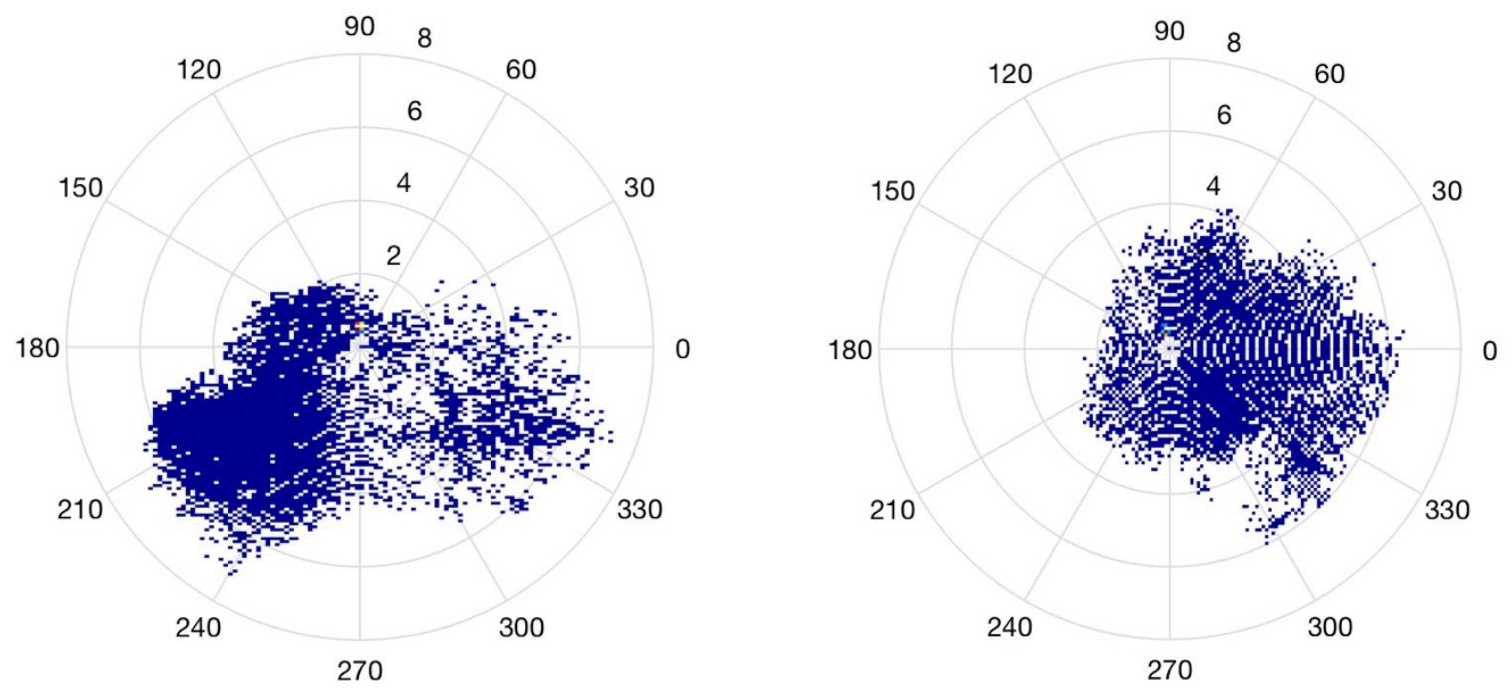

Figure 6-9. $5^{\text {th }}$ harmonic current polar heat plot at R3 [A]

Figure 6-10. $7^{\text {th }}$ harmonic current polar heat plot at $R 3$
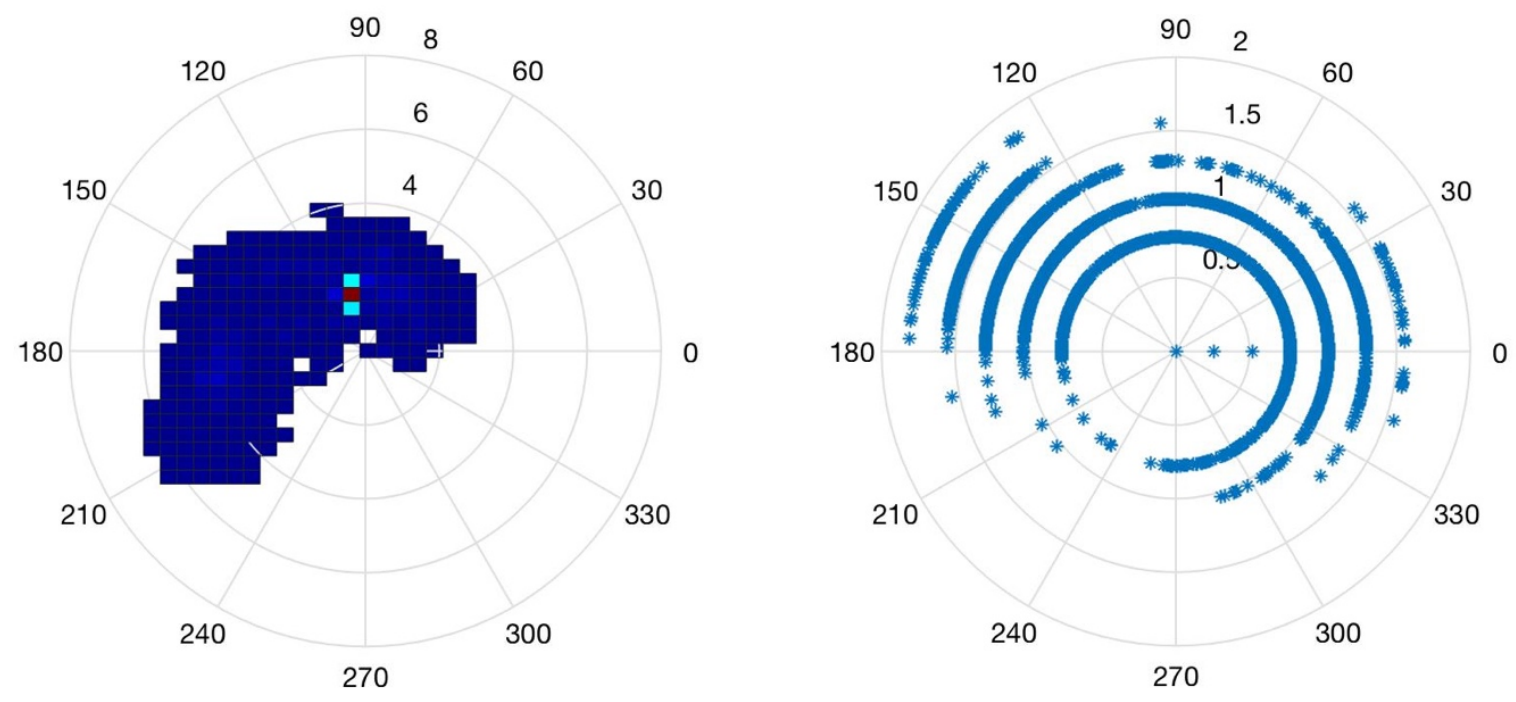

Figure 6-11. $5^{\text {th }}$ harmonic current polar heat plot at $R 4$ [A]

Figure 6-12. $7^{\text {th }}$ harmonic current polar plot at $R 4$ [A] 


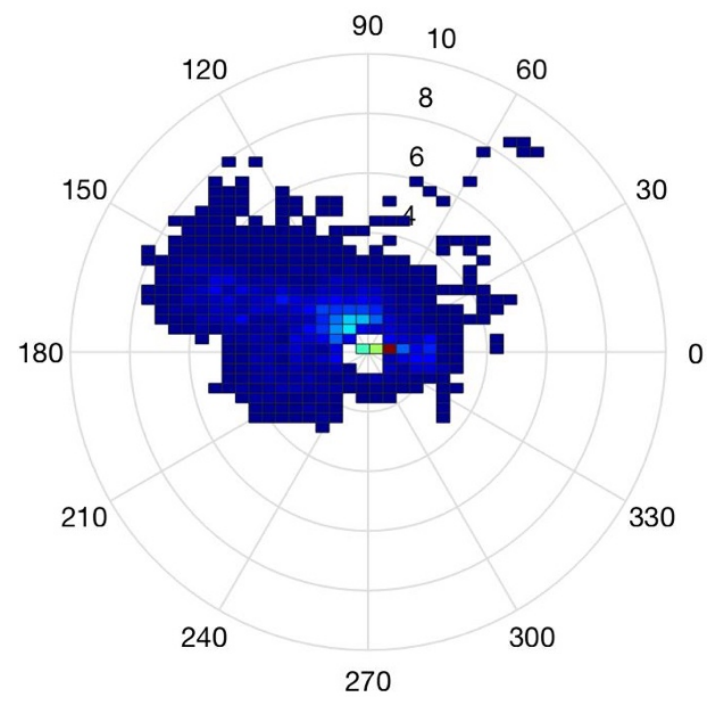

Figure 6-13. $5^{\text {th }}$ harmonic current polar heat plot at R5 [A]

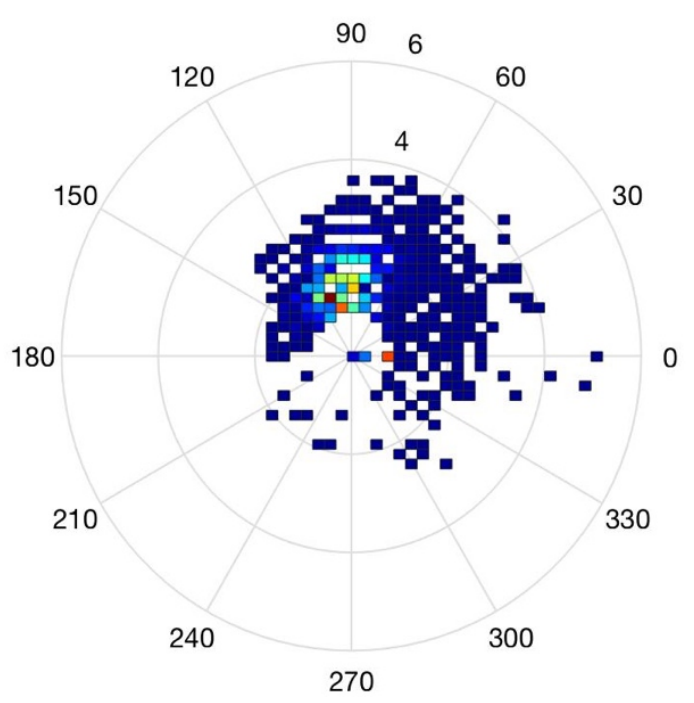

Figure 6-14. $7^{\text {th }}$ harmonic current polar heat plot at $R 5$ [A]

Polar heat plots for the measurement points R2 to R5 shows that all of the harmonic phasors operate in mostly all four quadrants highlighting the dynamic interaction of harmonic distortion between loads.

In the case of the PV plants at R3 and R4, the challenge will be to prove grid code compliance under these dynamic conditions. It is clear that the PV plants both emit and absorb harmonic distortion, where the grid code requires that the PV plants only account for what is emitted. As shown before, an rms value alone at a single measurement point cannot distinguish between harmonics which are being emitted and those being absorbed by the PV plant. Application of the general summation law as used in (2), will not result in an accurate analysis of the harmonic emission as the general summation law assumes static conditions as well as that harmonic orders summate in a certain fixed manner.

The level of prevalence is evaluated next with the application of (37) to the harmonic measurements. Note that the results presented consist of all of the data points with no separation of nighttime or daytime data. Recall from section 5.2 that [73] recommends that harmonic phase angle values should not be reported if the level of prevalence is below 0.8 .

Table 6-1. Level of prevalence for current harmonic measurement points R1 to R5

\begin{tabular}{|c|c|c|}
\hline Level of prevalence & $\mathbf{5}^{\text {th }}$ harmonic current & $\boldsymbol{7}^{\text {th }}$ harmonic current \\
\hline R1 & 0.2 & 0.5 \\
\hline R2 & 0.6 & 0.9 \\
\hline R3 & 0.6 & 0.5 \\
\hline R4 & 0.7 & 0.6 \\
\hline R5 & 0.7 & 0.9 \\
\hline
\end{tabular}

In Table 6-1 only two values are above 0.8 , hence the rest of the harmonic phase angle data would normally not be reported under this convention. These low levels of prevalence confirm the dynamic behaviour of harmonic emissions from the multiple nonlinear loads connected to the PCC at substation $\mathrm{B}$ as the harmonic current phasors are not static over time. They emphasise that harmonic emission compliance should also not be a once off task and that there should be continuous evaluation of harmonic emission from a renewable energy source or other nonlinear 
loads. Recall in section 5.4 how the harmonic emission did not stay constant and altered constantly depending on the PV plants generation output as well as changes in the upstream network which does not stay static.

Observe that the level of prevalence for the $5^{\text {th }}$ and $7^{\text {th }}$ harmonic at R1 are the lowest, this is due to the summation of all harmonics from the various nonlinear loads. For this measurement point, a single representative prevailing phase angle value should not be reported as a single value will not represent the entire $132 \mathrm{kV}$ network.

\subsection{Harmonic emission assessment of PV plants under dynamic conditions}

Harmonic emission calculations from the PV plants at measurement points R3 and R4 are discussed next. The aim is to apply various methods in the calculation of the harmonic emission from these two PV plants that will be used to test if the plant is compliant to their contractual obligations. Unfortunately, the actual harmonic emission limits are not available to the author as such no comment will be made if the plants meet their respective limits.

Firstly, the Range of Secondary Cancellation (RSC) technique in section 5.5.1 is applied to the measured data to only report the harmonic phasors which have a negative effect of the harmonic voltage at the PCC.

Secondly, as a comparison, the IEC method (section 2.4.2) is applied where the pre-connection and post-connection harmonic phasors are used to evaluate the emission.

Note: the IEC method requires that the PV plant be disconnected from the network completely to determine background harmonic voltage values, where the plant impedance does not cause reduction or amplification of the background harmonic voltages. However, due to commercial constraints, this was not done. The assumed pre-connection values are the values obtained when the PV plant is not producing (i.e. at night).

\subsubsection{RSC calculations for PV plants under dynamic conditions}

Table 6-2 list the calculated results as discussed in section 5.5.1 for the measurements at point R3.

Table 6-2. Calculated results for harmonic current RSC at R3

\begin{tabular}{|c|c|c|c|c|}
\hline & \multicolumn{2}{|c|}{$5^{\text {th }}$ harmonic } & \multicolumn{2}{|c|}{$7^{\text {th }}$ harmonic } \\
\hline & Mag & angle [degrees] & Mag & angle [degrees] \\
\hline $\begin{array}{l}\text { Background voltage distortion } \\
\boldsymbol{U}_{p r v, h, M V}[\mathrm{~V}]\end{array}$ & 573 & 128.8 & 247 & 203.5 \\
\hline Prevailing current $\boldsymbol{I}_{p r v, h}[\mathrm{~A}]$ & 4.17 & 47.6 & 1.9 & 186.8 \\
\hline Network impedance $\boldsymbol{Z}_{h}(\Omega)$ & 38.8 & 77.4 & 62.6 & 81.0 \\
\hline $\begin{array}{l}\text { Max range of cancellation } \\
\qquad \varphi_{I, p r v(\max )}\left[^{\circ}\right]\end{array}$ & & 313.3 & & 18.6 \\
\hline $\begin{array}{l}\text { Min range of cancellation } \\
\varphi_{I, p r v(\min )}\left[^{\circ}\right]\end{array}$ & & 149.6 & & 226.3 \\
\hline
\end{tabular}

Figure 6-15 to Figure 6-18 visualise the RSC results tabulated in Table 6-2 and Table 6-3. 

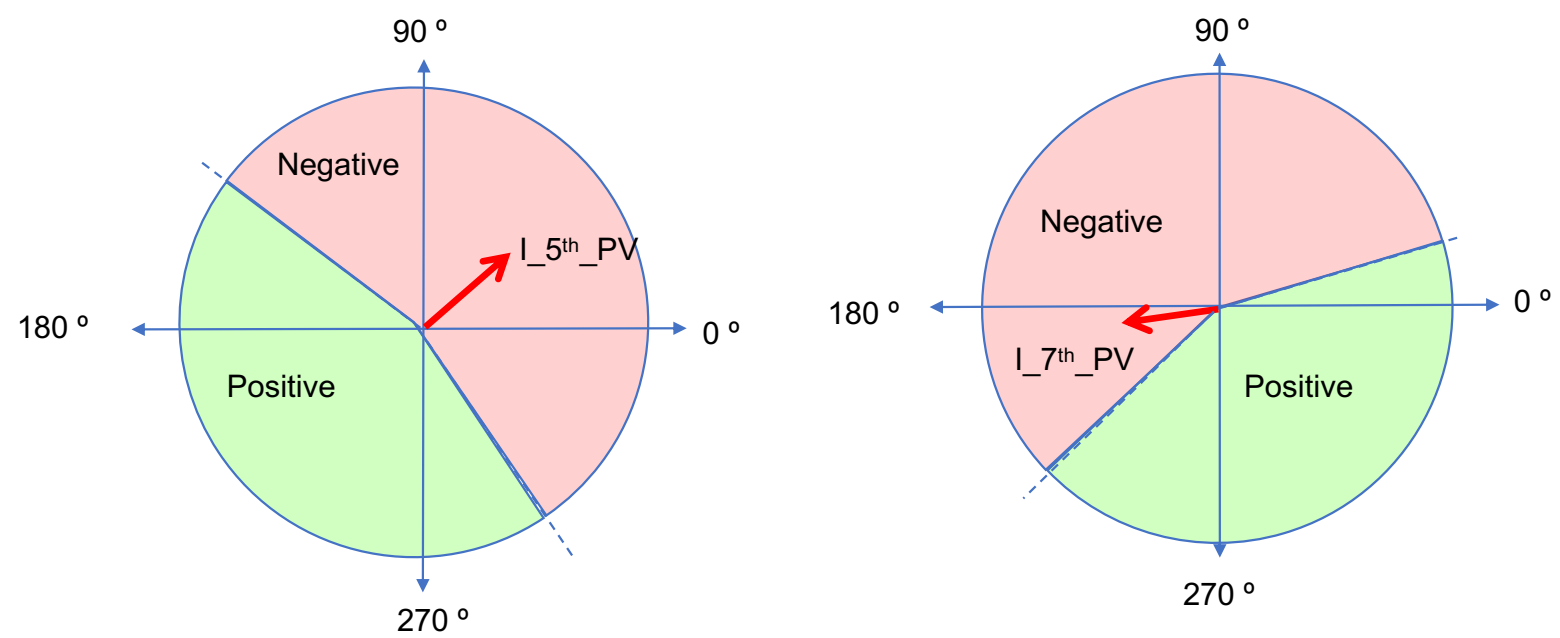

Figure 6-15. RSC for the $5^{\text {th }}$ harmonic current at $R 3$

Figure 6-16. RSC for the $7^{\text {th }}$ harmonic current at $R 3$

Table 6-3 list the calculated results as discussed in section 5.5.1 for the measurements at R4.

Table 6-3. Calculated results for harmonic current RSC at R4

\begin{tabular}{|c|c|c|c|c|}
\hline & \multicolumn{2}{|c|}{$5^{\text {th }}$ harmonic } & \multicolumn{2}{|c|}{$7^{\text {th }}$ harmonic } \\
\hline & Mag & angle [degrees] & Mag & angle [degrees] \\
\hline $\begin{array}{l}\text { Background voltage distortion } \\
\qquad \boldsymbol{U}_{p r v, h, M V}[\mathrm{~V}]\end{array}$ & 608 & 125.9 & 249.4 & 206.2 \\
\hline Prevailing current $\boldsymbol{I}_{p r v, h}[\mathrm{~A}]$ & 4.3 & 354.5 & 0.9 & 290.8 \\
\hline Network impedance $\boldsymbol{Z}_{h}(\Omega)$ & 38.8 & 77.4 & 62.6 & 81.0 \\
\hline $\begin{array}{l}\text { Max range of cancellation } \\
\qquad \varphi_{I, p r v(\max )}\left[^{\circ}\right]\end{array}$ & & 310.7 & & 28.9 \\
\hline $\begin{array}{l}\text { Min range of cancellation } \\
\qquad \varphi_{I, p r v(\min )}\left[^{\circ}\right]\end{array}$ & & 146.4 & & 221.5 \\
\hline
\end{tabular}

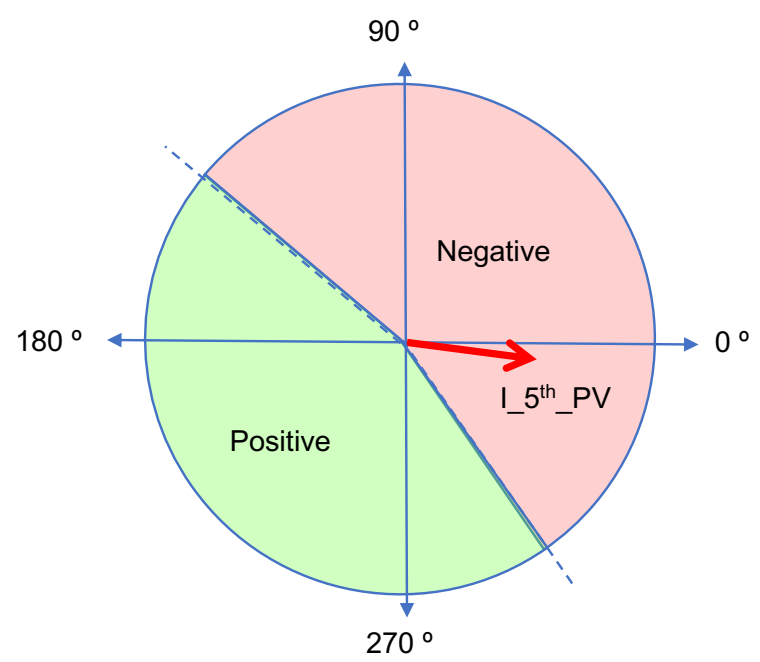

Figure 6-17. RSC for the $5^{\text {th }}$ harmonic current at $R 4$

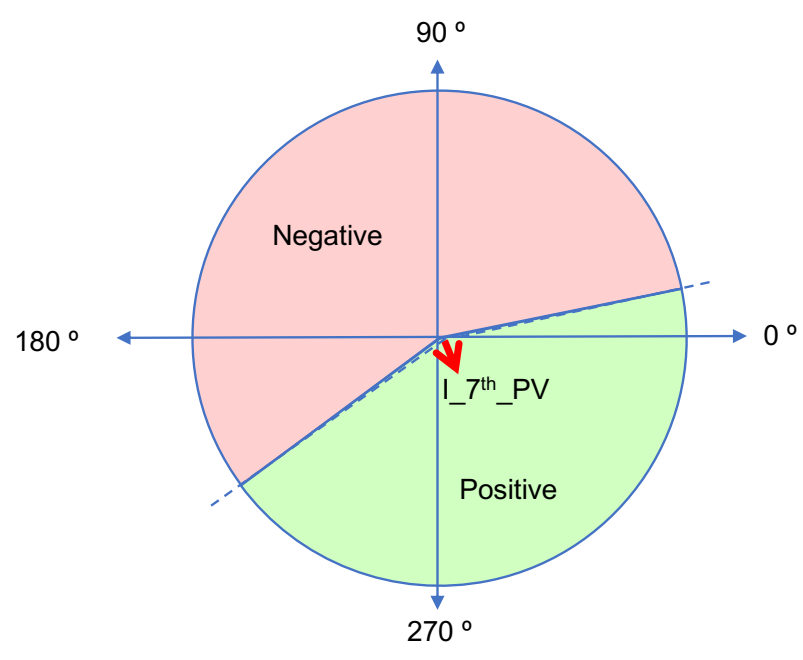

Figure 6-18. RSC for the $7^{\text {th }}$ harmonic current at $R 4$ 
Figure 6-15 to Figure 6-18 shows the RSC angles are in the same angular range for both PV plants. This is due to the PV plants seeing the same background voltage harmonics (same PCC) and the same network harmonic impedance.

Operation of the $5^{\text {th }}$ and $7^{\text {th }}$ harmonic current prevailing phasors for PV Plant A (measurement point R3) shown in Figure 6-15 and Figure 6-16 as a red arrows, are in the negative area of the RSC. The PV plant thus has a negative impact on both the $5^{\text {th }}$ and $7^{\text {th }}$ harmonic voltages at the PCC when the plant is in operation.

The $5^{\text {th }}$ harmonic current prevailing phasor for PV Plant B (measurement point R4) shown in Figure 6-17, operates in the negative RSC indicating that the $5^{\text {th }}$ harmonic current has a negative impact on the $5^{\text {th }}$ harmonic voltage. In contrast the $7^{\text {th }}$ harmonic current prevailing phasor shown in Figure 6-18 operates in the positive RSC indicating that the PV plant has a positive impact on the $7^{\text {th }}$ harmonic voltage.

The two PV plants are the same size, yet the harmonic emission is different. Possible reasons for this are as follows:

- The equipment that they deployed are from different manufacturers,

- The inverters from PV plant B may be better optimised for harmonic emission. Note that the latter cannot be confirmed since the information is not available.

It is the intention of a harmonic emission assessment to cater for only those harmonic distortion values that are emitted onto the network, i.e. that have a negative effect on the harmonic voltage at the PoC. The RSC technique is used to only report those harmonic current values that operate in the negative area of Figure 6-15 to Figure 6-18 to ensure that only values deemed as harmonic emission is taken into account for the harmonic emission calculations. Figure 6-19 to Figure 6-22 shows the aggregated harmonic phasor values which will be used in the harmonic emission calculation, which is discussed in the next section.

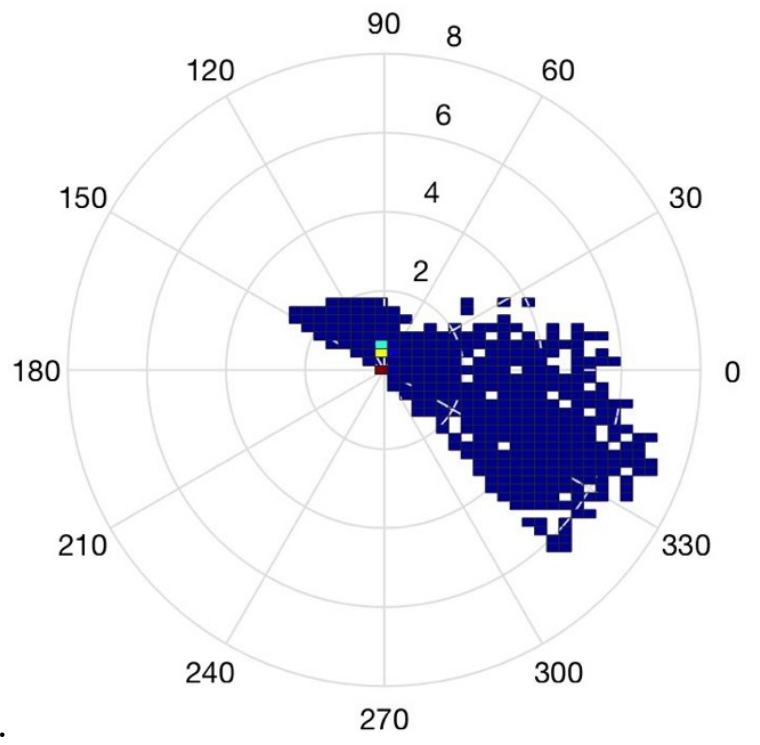

Figure 6-19. $5^{\text {th }}$ harmonic current aggregated phasor values operating in negative RSC at $R 3$ [A]

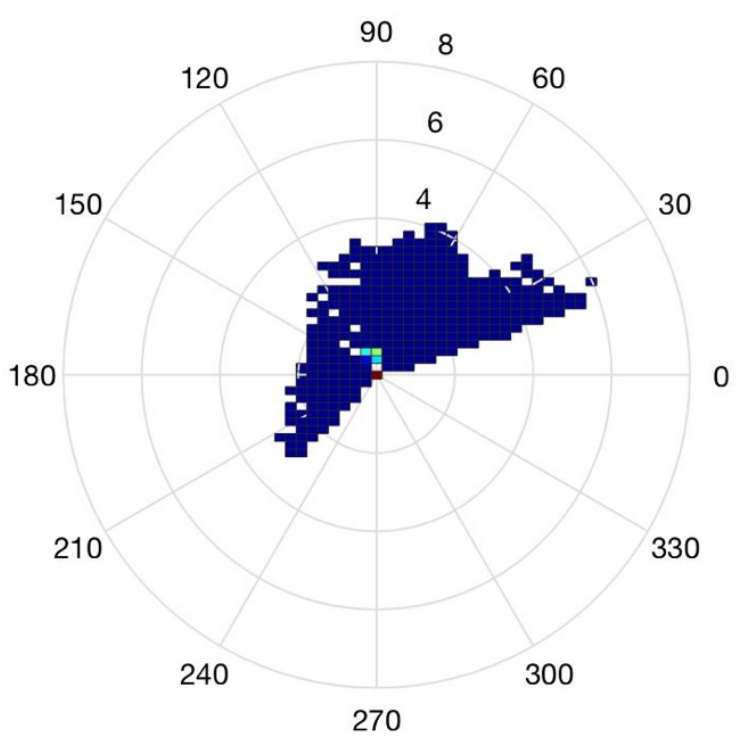

Figure 6-20. $7^{\text {th }}$ harmonic current aggregated phasor values operating in negative RSC at $R 3$ [A] 


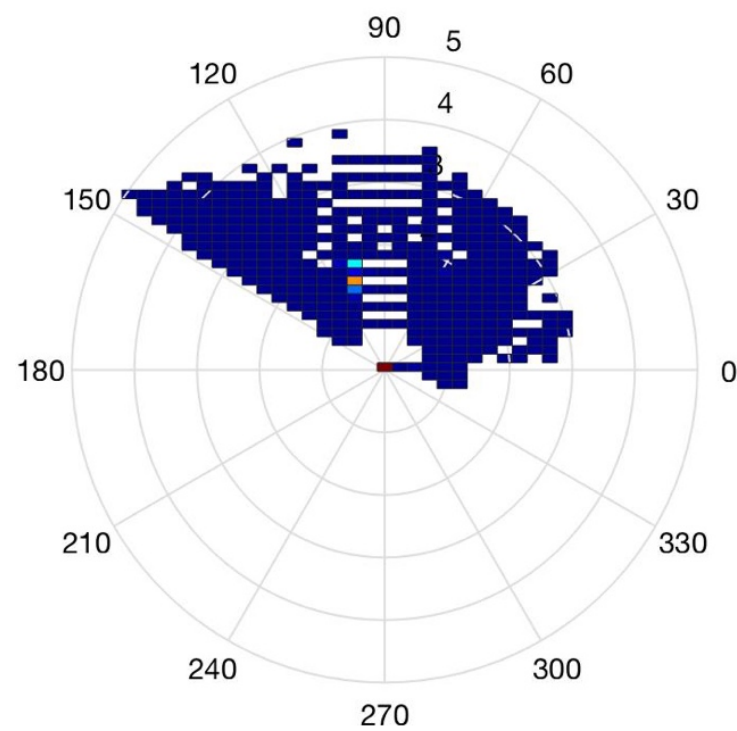

Figure 6-21. $5^{\text {th }}$ harmonic aggregated phasor values operating in negative RSC at R4 [A]

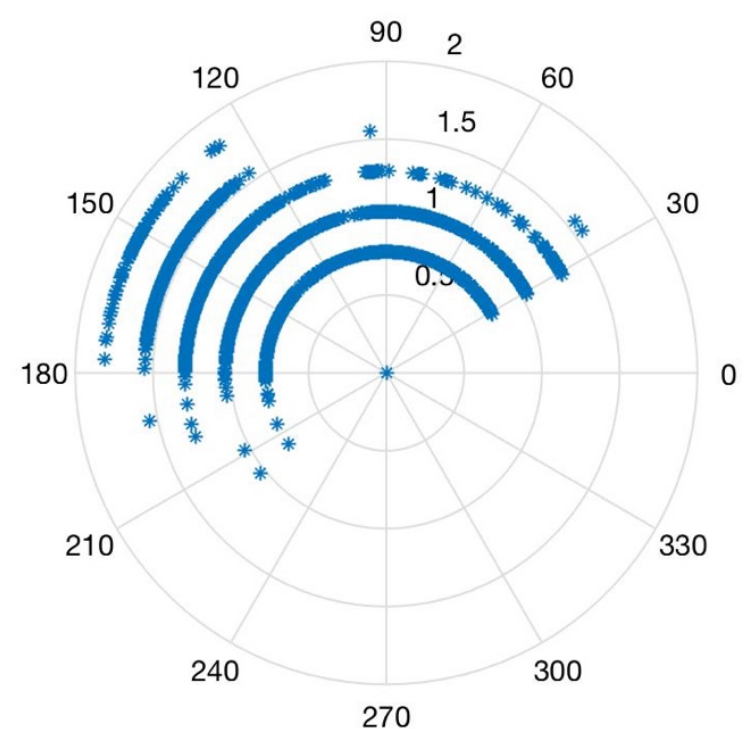

Figure 6-22. $7^{\text {th }}$ harmonic aggregated phasor values operating in negative RSC at R4 [A]

\subsubsection{A comparison between the prevailing phase angle method and the IEC method for harmonic emission calculations.}

In this section the harmonic emission values are calculated that should be compared to the contractual limits as issued by the DSO. Results from the prevailing angle methodology and the IEC general summation [4] law is firstly compared by using all of the available data and then secondly using the discriminated data which is considered to be emission only.

To ensure comparable results, a single day is used for all the calculations. Figure 2-1 is used a refence for the calculation of the harmonic emission component. The data was split into a generating period (post connection) and a non-generating period (pre connection). For the IEC method, the $95^{\text {th }}$ percentile [7], [41] of the generating period was taken as a representative data point and similarly for the non-generating period with the harmonic emission calculation done using (2). The prevailing phase angle is calculated using (35) as the representative a data point for the generating and non-generating conditions with the emission value taken as a phasor subtraction of the post and pre connections values.

Table 6-4 list the results of the IEC method and the prevailing phase angle method. Comparing the results of the two methods, the values for the non-generating period are very similar to each as expected, as only the background values are considered (any influence of the PV plant being connected is ignored).

Table 6-4. Harmonic emission results from PV Plant at $R 3$ with all data considered.

\begin{tabular}{|c|c|c|c|c|c|c|c|}
\hline \multirow{2}{*}{ Emission calculation } & \multicolumn{2}{|c|}{ Non-generation } & \multicolumn{2}{c|}{ Generating } & \multicolumn{2}{c|}{ Emission } \\
\cline { 3 - 8 } \multicolumn{2}{|c|}{} & Mag [A] & $\begin{array}{c}\text { Phase } \\
\text { [Degrees] }\end{array}$ & Mag [A] & $\begin{array}{c}\text { Phase } \\
\text { [Degrees] }\end{array}$ & Emission [A] & $\begin{array}{c}\text { Phase } \\
\text { [Degrees] }\end{array}$ \\
\hline \multirow{2}{*}{$5^{\text {th }}$ harmonic } & IEC method & 0.7 & & 5.3 & & 5.1 & \\
\cline { 2 - 8 } & Prevailing & 0.6 & 95.0 & 4.4 & -132.5 & 4.6 & -126.8 \\
\hline \multirow{2}{*}{$7^{\text {th }}$ harmonic } & IEC method & 0.5 & & 3.8 & & 3.2 & \\
\cline { 2 - 8 } & Prevailing & 0.6 & 99.1 & 1.9 & 6.5 & 2.0 & 169.2 \\
\hline
\end{tabular}


The generating period values for the two methods are different as the IEC method took a statistical approach in the $95^{\text {th }}$ percentile value, which may not always represent the dynamic data set as recorded. The prevailing phase angle method, however, took all of the data points into consideration for the generating period and aggregated them to result in a single value for an improved data set representation.

Harmonic emission calculation using the prevailing phase angle method is lower than the IEC method. This is mainly due to the use of a summation factor in the IEC calculation, while the prevailing phase angle makes use of measured aggregated phase angles per harmonic order.

Table 6-5 list the harmonic emission values of the PV plant at R4. The prevailing phase angle method again resulted in lower values than the IEC method.

Table 6-5. Harmonic emission results from PV Plant at R4 with all data considered.

\begin{tabular}{|c|c|c|c|c|c|c|c|}
\hline \multirow{2}{*}{\multicolumn{2}{|c|}{ Emission calculation }} & \multicolumn{2}{|c|}{ Non-generation } & \multicolumn{2}{|c|}{ Generating } & \multicolumn{2}{|c|}{ Emission } \\
\hline & & $\operatorname{Mag}[A]$ & Phase [ $\left.{ }^{\circ}\right]$ & $\operatorname{Mag}[A]$ & Phase [ 10 & Emission [A] & Phase ["] \\
\hline \multirow{2}{*}{$5^{\text {th }}$ Harmonic } & IEC method & 2.1 & & 6.0 & & 5.0 & \\
\hline & Prevailing & 1.7 & 101.0 & 4.3 & 174.5 & 4.1 & -162.2 \\
\hline \multirow{2}{*}{$7^{\text {th }}$ Harmonic } & IEC method & 1.0 & & 1.3 & & 0.5 & \\
\hline & Prevailing & 1.0 & 92.4 & 0.9 & 114.9 & 0.4 & -146.5 \\
\hline
\end{tabular}

Using the same day for the harmonic emission calculations as above, the exercise is repeated but only using the data as displayed in Figure 6-19 to Figure 6-22 ,i.e. the data in the positive RSC region is removed.

Table 6-6 list the harmonic emission results for the PV plant at R3. Considerable reductions in the emission values for both methods are observed especially with the use of the prevailing phase angle method.

Table 6-6. Harmonic emission results from PV Plant at $R 3$ with only discriminated data considered

\begin{tabular}{|c|l|c|c|c|c|c|c|}
\hline \multirow{2}{*}{ Emission calculation } & \multicolumn{2}{|c|}{ Non-generation } & \multicolumn{2}{c|}{ Generating } & \multicolumn{2}{c|}{ Emission } \\
\cline { 3 - 8 } \multicolumn{2}{|c|}{} & Mag [A] & Phase ['] & Mag [A] & Phase [\%] & Emission [A] & Phase [\%] \\
\hline \multirow{2}{*}{$5^{\text {th }}$ harmonic } & IEC method & 0.7 & & 4.7 & & 4.5 & \\
\cline { 2 - 8 } & Prevailing & 0.6 & 93.2 & 1.9 & -30.8 & 2.3 & -43.0 \\
\hline \multirow{2}{*}{$7^{\text {th }}$ harmonic } & IEC method & 0.5 & & 3.0 & & 2.8 & \\
\cline { 2 - 8 } & Prevailing & 0.6 & 100.5 & 1.3 & 84.7 & 0.7 & 72.0 \\
\hline
\end{tabular}

Table 6-7 list the harmonic emission results for the PV plant at R4. Once again, a drastic reduction in the harmonic emission values are seen when comparing the two applied methods, except for the harmonic emission the $7^{\text {th }}$ harmonic. The prevailing phase angle emission value is slightly higher than the IEC value. 
Table 6-7. Harmonic emission results from PV Plant at R4 with only discriminated data considered

\begin{tabular}{|c|c|c|c|c|c|c|c|}
\hline \multirow{2}{*}{\multicolumn{2}{|c|}{ Emission calculation }} & \multicolumn{2}{|c|}{ Pre } & \multicolumn{2}{|c|}{ Post } & \multicolumn{2}{|c|}{ Emission } \\
\hline & & $\operatorname{Mag}[A]$ & Phase $\left[{ }^{\circ}\right]$ & $\operatorname{Mag}[A]$ & Phase [ $\left.{ }^{\circ}\right]$ & Emission [A] & Phase $\left[{ }^{\circ}\right]$ \\
\hline \multirow{2}{*}{$5^{\text {th }}$ harmonic } & IEC method & 2.1 & & 3.6 & & 2.3 & \\
\hline & Prevailing & 1.7 & 101.0 & 1.8 & 116.4 & 0.5 & -165.7 \\
\hline \multirow{2}{*}{$7^{\text {th }}$ harmonic } & IEC method & 1.0 & & 1.3 & & 0.5 & \\
\hline & Prevailing & 1.0 & 92.4 & 0.8 & 124.9 & 0.6 & -143.5 \\
\hline
\end{tabular}

\subsection{Conclusion}

Application of aggregated harmonic phasors was limited to low voltage and medium voltage networks as the harmonic phasors are considered to me more stable at these lower voltage levels. Nothing could be found in literature on the application of this novel method to a high voltage network.

In an attempt to better understand the complications of harmonic emission assessment on a high voltage network, five PQ instruments were deployed to measure the aggregated harmonic phasors for both voltage and current. The instruments were GPS synchronised to ensure that each recorder measured the same load and network conditions at the same instant in time for comparable results.

A further complication in the measurement campaign was the presence of multiple nonlinear loads connected to the same $132 \mathrm{kV}$ busbar, which presented an opportunity to evaluate the dynamic behaviour of harmonic distortion at this busbar by using aggregated harmonic phasor measurements.

Results presented show the widespread orientation of harmonic phasors for nonlinear loads, where in some cases the harmonic phasors operated in all four quadrants of a polar plot. This was supported by the low levels of prevalence for the respective measurement points.

According to literature, which was based on lab-generated data [80], low levels of prevalence should not be reported due to the high variation of the harmonic phasor within the considered timeframe, but in the high voltage application it was shown that the low level of prevalence highlights the dynamic behaviour of harmonic distortion when all of the available data is considered. The level of prevalence can be improved when shorter time periods are considered or to only focus on certain operating modes of the nonlinear load, for example when the PV plant is in generating energy and when it is not.

Renewable energy sources undergoing grid code compliance must only account for the harmonic emissions that they emit onto the network. It was always thought that harmonic distortion tends to flow towards the upstream network [55] but literature has shown that this is not always the case [16]. Harmonic distortion flows between nonlinear loads connected onto the same busbar and do not summate in a fixed manner.

Harmonic emissions of the two PV plants under investigation were firstly calculated using the prevailing phase angle method for all data points and secondly compared to the IEC general summation law. Secondly, the RSC method was applied to the raw data set to remove harmonic current values, which have a positive impact on the harmonic voltage distortion at the PCC under investigation.

Pre-connection values were taken when the individual plants are not generating electrical energy and the post connection is taken when the PV plants are in generation mode. 
Results of the first method show that the IEC method is conservative due to the use of a summation exponent that assumes that the harmonic distortion adds in a certain manner and the use of a $95^{\text {th }}$ percentile value when only rms values are considered.

Use of the prevailing phase angle method makes use of the entire data set to result in a representative index (consisting of a magnitude and phase angle) which is then used for the harmonic emission. Results of the harmonic emission assessment using this method yielded a lower harmonic emission value as representative phase angle information was used.

Results of the second method show a considerable reduction in the harmonic emission values. Differences in the emission values of the two methods were also seen except for the $7^{\text {th }}$ harmonic at R4 where the emission value was slightly higher than the IEC method.

The prevailing phase angle method thus yields a harmonic emission value which is representative of a dataset which may have varying dynamic aspects embedded within the results, such as changes in the network harmonic impedance due to upstream network contingencies or changes in the cloud cover (in the case of a PV plant). Harmonic emission assessment is further improved when only data that has a negative effect on the upstream network is considered, again showing a lower harmonic emission value compared to the IEC method. 


\section{CHAPTER 7: Consideration on the aggregation time interval}

\subsection{Introduction ${ }^{11}$}

In Chapter 5 it was shown, through the harmonic emission assessment of a PV plant, that the harmonic emissions from this non-linear load is not static and can change from one measurement sample to the next. These changes are typically dependent on the solar irradiation in terms of cloud cover or changes in the upstream network configuration. Depending on the configuration of PQ measurement instrument used, these harmonic emission changes may not be captured.

A measurement period of 7 days at 10-min rms values is recommended by the South African grid code for proving PQ compliance [1]. Harmonic emissions from non-linear loads, such as Renewable Power Plants (RPP), is dynamic and a 7 day measurement period may not fully represent the harmonic emission profile of a non-linear load. Ideally harmonic emission assessment should be done on a continuous basis to ensure that RPPs remain within their contractual obligations as well as to ensure that PQ compatibility levels are maintained by the DSO. The aggregation time interval is one of the key factors that must be considered when continuous measurements are to be done.

While the impact of the aggregation time interval has been done for rms values [105], [106], no study on the aggregation time interval of the related phase angle information had been found. This chapter aims to firstly evaluate the impact of the time aggregation interval on harmonic phasors by means of a field application at two high voltage sites where harmonic phase angles vary significantly at one site and an almost stable harmonic phase angle at the other site. Secondly to develop a method that will ensure that each event is captured at the highest possible time interval without compromising the measurement dataset while still ensuring a reduced dataset for easier analysis and longer measurement periods.

The majority of modern-day PQ instruments that are compliant to IEC 61000-3-40 have the capability to store data at selectable aggregation time intervals. Standard time intervals are 200 ms, 1-min, 10-mins and 2-hours. Default aggregation time interval on PQ instruments is 10-mins, as PQ standards such as EN 50160 [41] and NRS 048 [7] use this time aggregation interval value to benchmark PQ performance levels.

\subsection{Evaluation of time aggregation intervals}

1-min aggregated harmonic phasor measurements were taken at two $132 \mathrm{kV}$ sites with different network topologies and with different dynamics pertaining to the types of loads connected. Site A was taken as the PV plant connected to R3 in Figure 6-1, Site B is shown in Figure 7-1 which supplies a large reticulation network with small industrial and farming loads. A minimum of two weeks data was measured at each site and all of the available data, unless otherwise stated, was used for this evaluation. Analysis of the measured harmonic phase angles at these two sites highlighted the different phase angles operations and dynamics, which was deemed suitable for the research into aggregation time intervals.

Using the aggregation principles of IEC 61000-4-30 [49], as discussed in section 5.2 the measured data at the two sites are aggregated from the 1-min values to 10-min and 120-minute data sets. Only the $5^{\text {th }}$ harmonic current phase angle information will be analysed in this section, as the

\footnotetext{
${ }^{11}$ One conference paper has been published based on the research presented in this chapter [116].
} 
evaluation is on the impact of time aggregation and not the harmonic emission analysis of the two sites. The $5^{\text {th }}$ harmonic current further showed sufficient variability to illustrate the hypothesis.

Figure 6-9 (repeated below) and Figure 7-2 show the polar heat plots of the two high voltage sites under investigation. Observe that aggregated harmonic current phasors at Site A are widespread and operating in all four quadrants, while Site B is operating mainly in quadrant 2 and shows almost static behaviour.

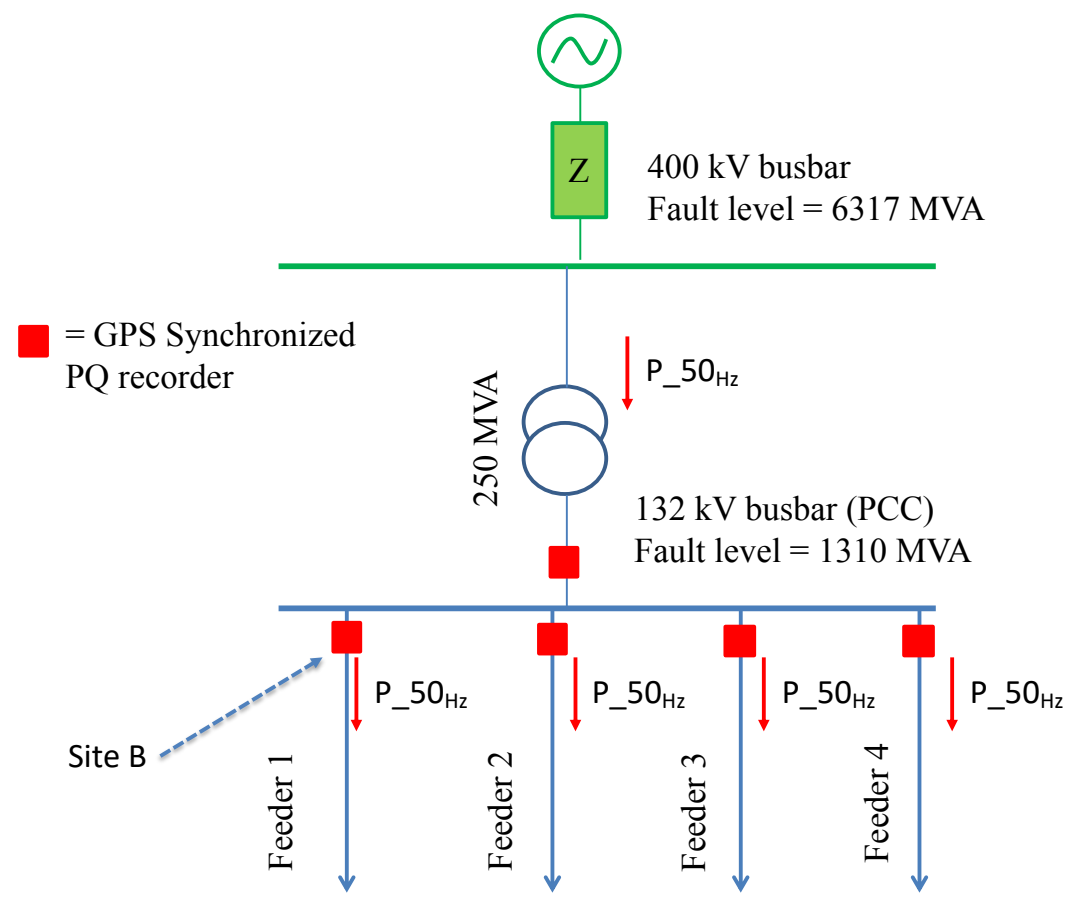

Figure 7-1. Location of Site B PQ recorder in high voltage network.

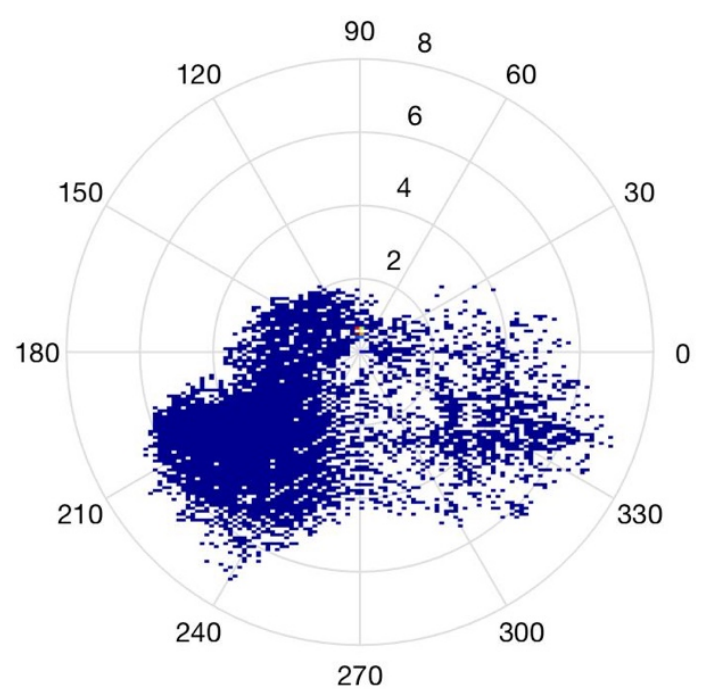

Figure 6-9. 5th harmonic current polar heat plot at R3 [A]

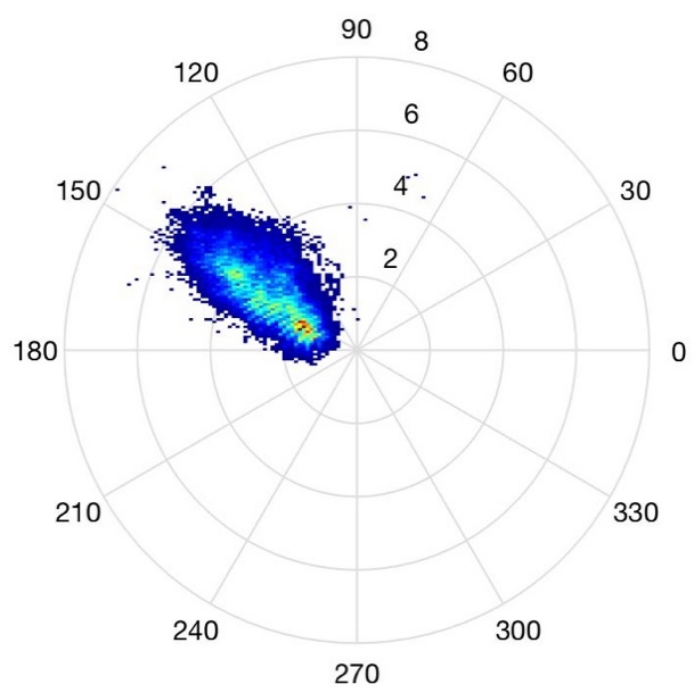

Figure 7-2. $5^{\text {th }}$ harmonic current polar heat plot for Site $B$.

Figure 7-3 shows the harmonic current phase angle aggregation time interval plots for Site $\mathrm{A}$. The first time plot shows the measured 1-min aggregation plot, the middle plot shows the 10-min aggregation plot and the third plot shows the 120-min aggregation plot. 

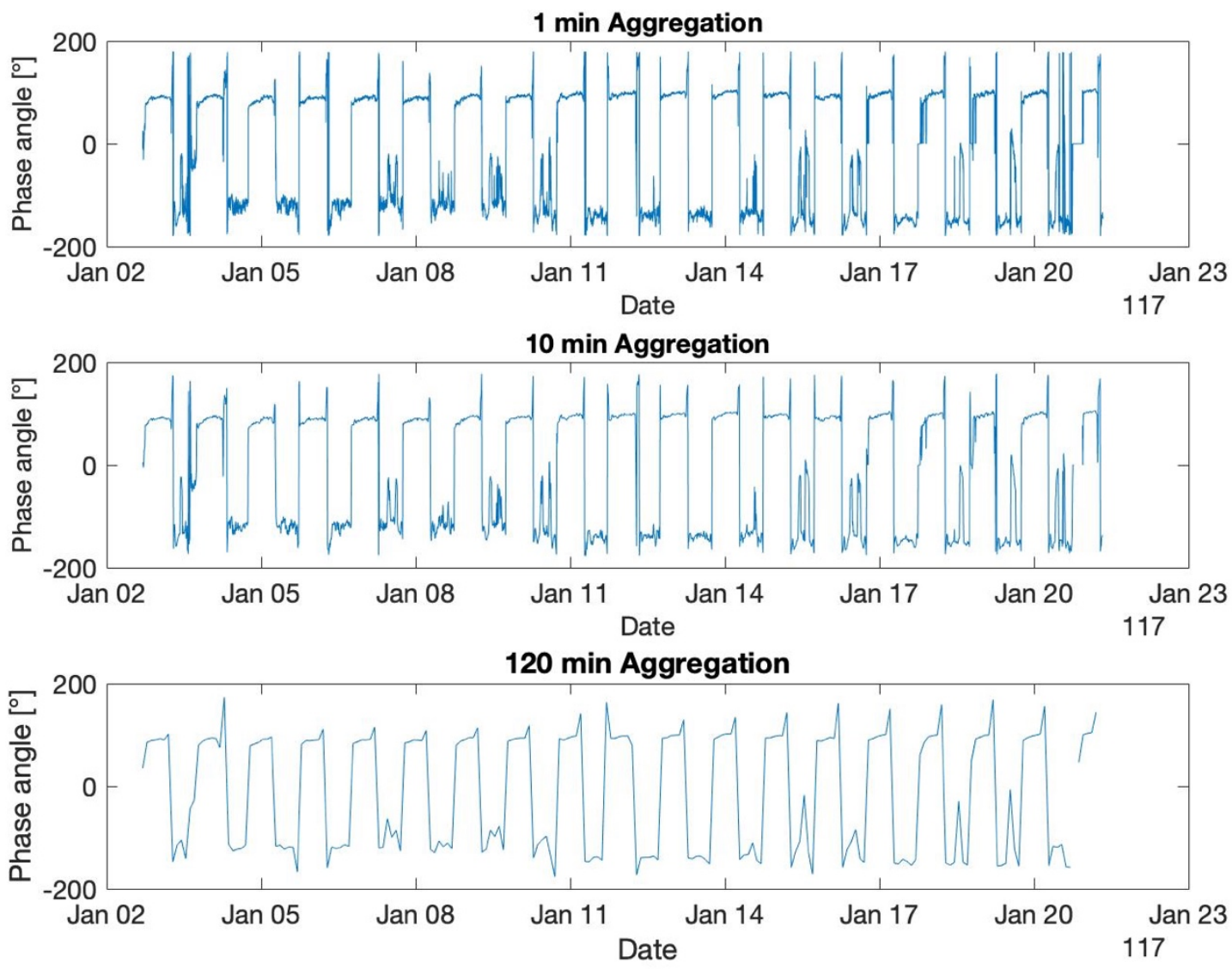

Figure 7-3. Aggregation time interval plots for Site A (R3), all data

Figure 7-3 shows the dynamic harmonic behaviour of a PV plant where the day and nighttime changes in the current harmonic phase angle can be seen.

The daytime operation of the PV plant is around the $-150^{\circ}$ area while the nighttime operation is around $100^{\circ}$. Previous harmonic emission analysis on a PV plant in section 5.4 has shown that changes in the harmonic phase angle may be due to changes in cloud cover or changes in the upstream network (as example) resulting in a sudden change in the harmonic emission direction. In Figure 7-3, these changes in the harmonic phase angle on the 1-min and 10-min profiles display the impact of cloud cover, as example, as the phase angles do not always remain around the $-150^{\circ}$ as observed around $15 \mathrm{Jan}$.

Observe the changes in resolution between the 1-min, 10-min and 120-min aggregation plots in Figure 7-3. As the aggregation time interval is increased, the detailed variation of the data presented in the chart is decreased, i.e. the plot is smoothed out. In the 120-min plot, only the change between nighttime and daytime is visible with small "cloud cover" changes (as example) supressed or "smoothed" out in the dataset due to the aggregation process. Figure 7-4 shows the aggregated data for one complete day to better observe the "smoothing" of the data. 

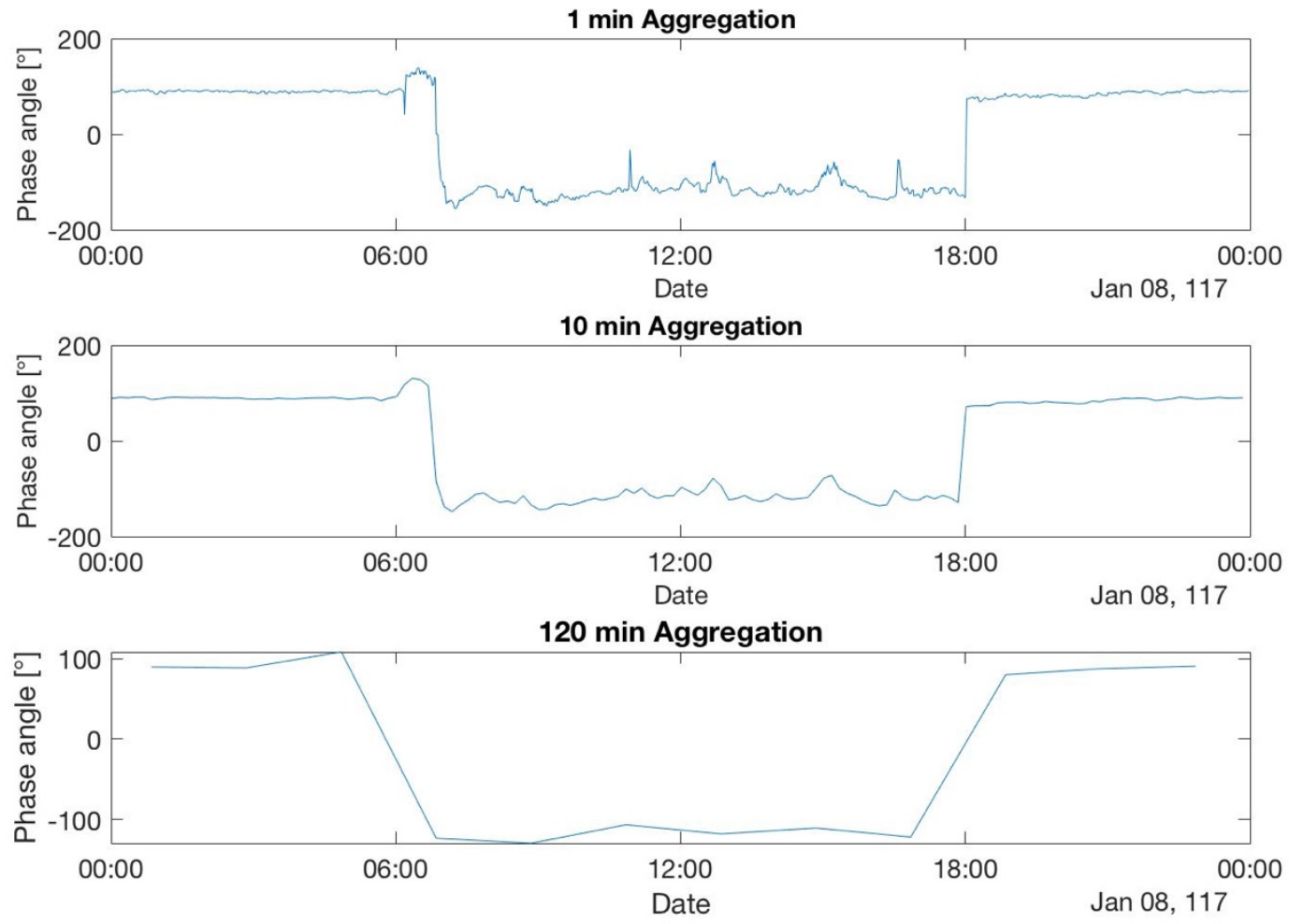

Figure 7-4. Aggregation time interval plots for Site A (R3), one day's data

Figure 7-5 shows the aggregation time interval plots for Site B. Due to the "static" nature of the harmonic phasors, the impact of changing the aggregation time interval does not yield much change in the harmonic phase angle profiles. The harmonic phase angle remains around $150^{\circ}$ for different aggregation intervals.
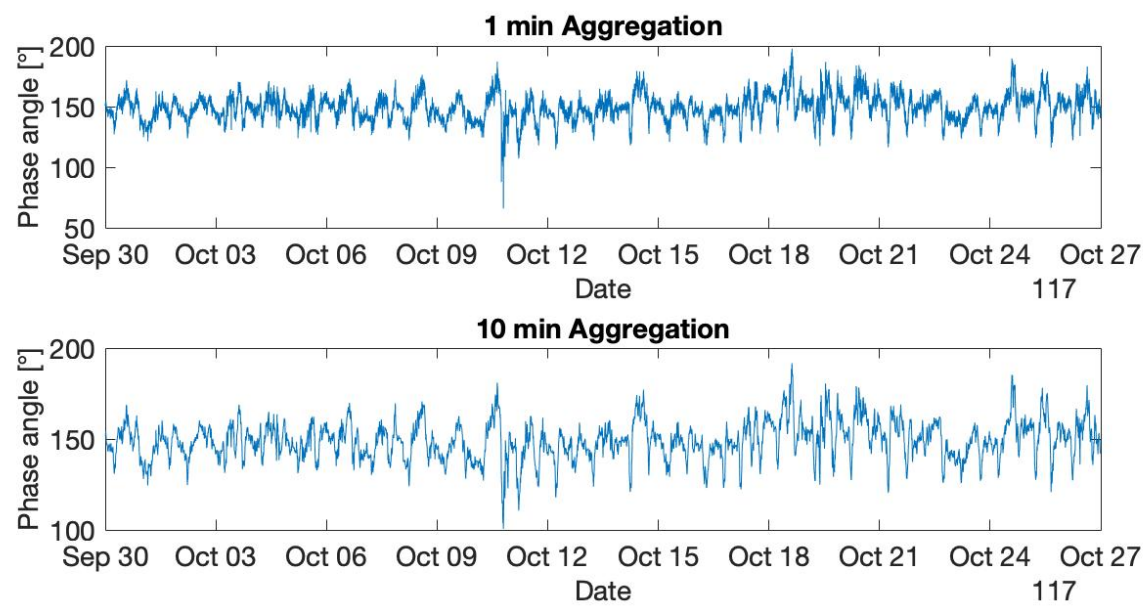

120 min Aggregation

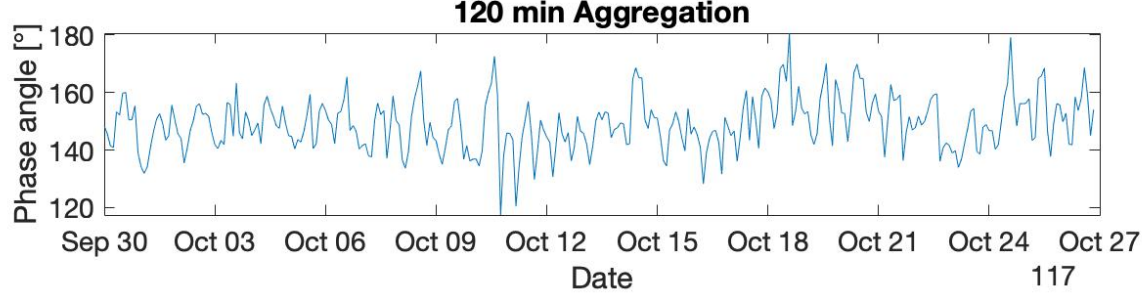

Figure 7-5. Aggregation time interval plots for Site B, all data 
Histograms of the harmonic phasor angles are plotted in Figure 7-6 and in Figure 7-7 for the aggregation time intervals presented in Figure 7-3 and Figure 7-5. The plots show the reduction in the number of data points due to the aggregation process, when comparing the number of data points on each chart. Number of data points reduced from thousands of points in the 1-min aggregation to hundreds of points for the 10-min aggregation and then to the tens for the 120-min aggregation.

In Figure 7-6, the histogram shows bimodal operation for the PV plant. From Figure 7-3 and Figure 7-6, it is inferred that the two modes represent the daytime and night-time operation of the PV plant respectively. The $5^{\text {th }}$ harmonic current phase angle is centred around $-130^{\circ}$ during daytime operation and centred around $90^{\circ}$ during night-time operation.
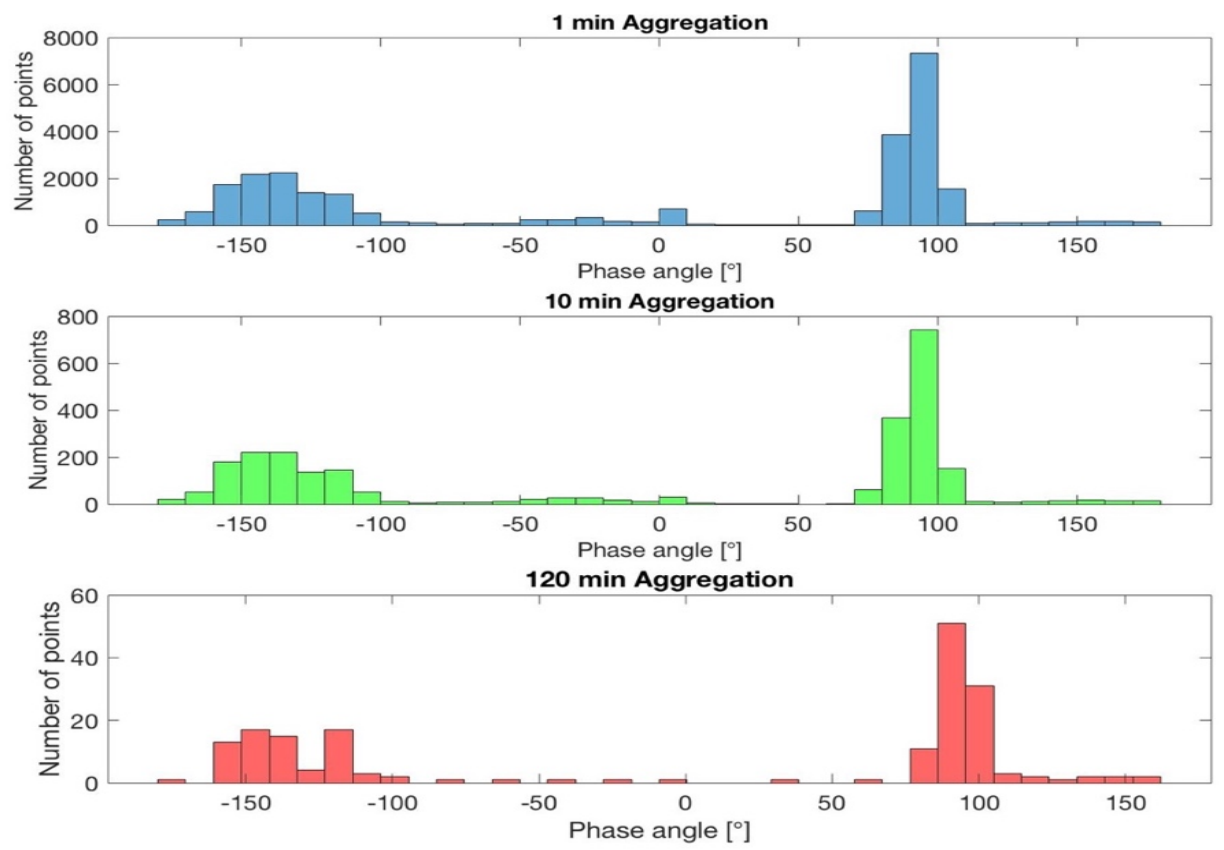

Figure 7-6. Aggregation time interval plots for Site A (R3)
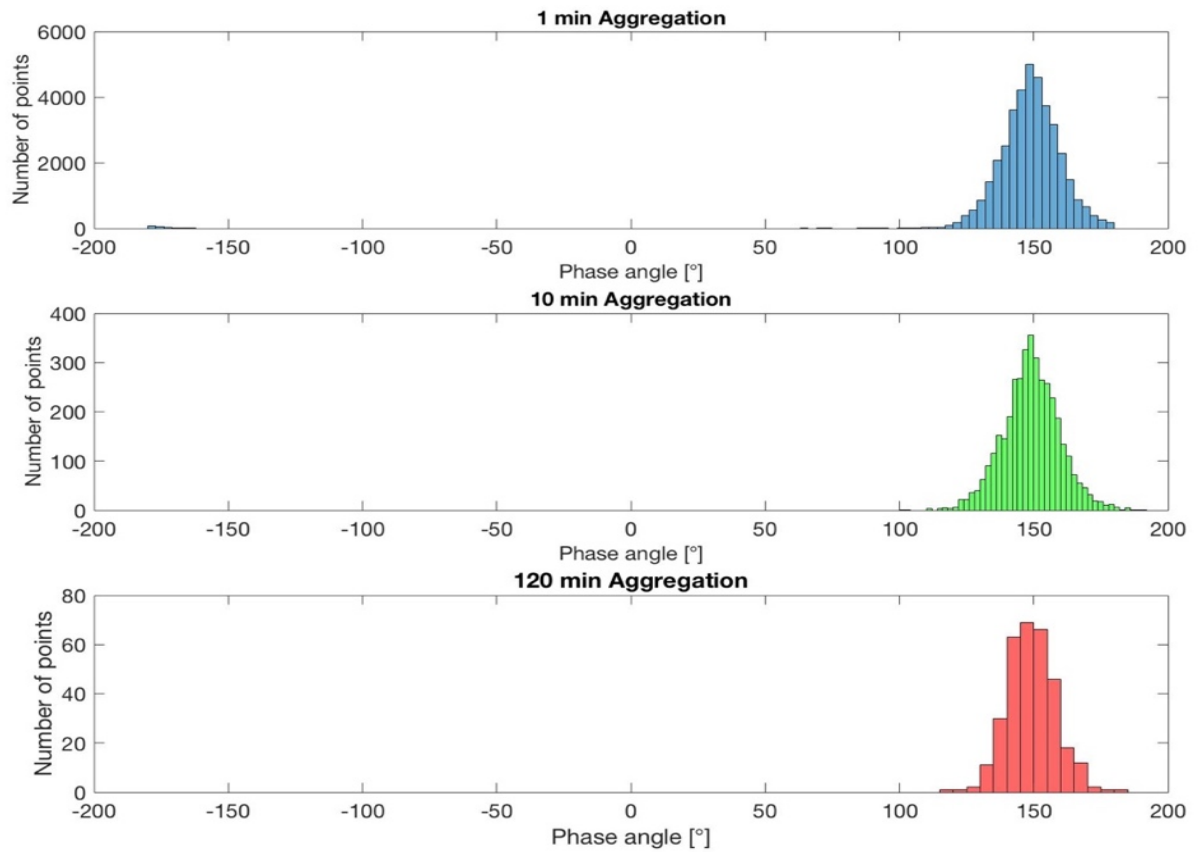

Figure 7-7. Aggregation time interval plots for Site B 
Figure 7-7 shows one mode of operation for Site A emphasising the stability of the harmonic phase angle irrespective of the aggregation time period used.

\subsection{Impact on level of prevalence}

Level of prevalence for the aggregated datasets is listed in Table 7-1. This analysis is done to determine if there is an impact on the harmonic phase angle variation as the aggregation time interval is increased from 1-min to 120-min aggregation intervals.

Table 7-1. Level of prevalence for Site A and Site B for different aggregation time intervals

\begin{tabular}{|c|c|c|}
\hline \multirow{2}{*}{ Aggregation interval } & \multicolumn{2}{|c|}{$\begin{array}{c}\text { Level of prevalence: } 5^{\text {th }} \text { harmonic current } \\
\text { phase angle }\end{array}$} \\
\cline { 2 - 3 } & Site $\boldsymbol{A}$ & Site $\boldsymbol{B}$ \\
\hline 1-min & 0.59 & 0.99 \\
\hline $10-\mathrm{min}$ & 0.60 & 0.99 \\
\hline $120-\mathrm{min}$ & 0.64 & 0.99 \\
\hline
\end{tabular}

Observe the low levels of prevalence for Site A shown in Table 7-1, in comparison to Site B shown in Table 7-2. According to [73], [80] a prevalence level of less than 0.8 should not be reported, but the analyses done in Chapter 3: and Chapter 5: highlight the dynamic behaviour of harmonic distortion.

The aggregation time interval does not significantly change the level of prevalence at either Site A or Site B.

As an impact study, the harmonic prevailing phasor is calculated using (34) for the complete datasets at the different aggregation time intervals. The aim is to evaluate if there is an impact on the results, should a representative value be required.

Table 7-2 Prevailing phasor values for different aggregation time intervals

\begin{tabular}{|c|c|c|c|c|}
\hline \multirow{2}{*}{$\begin{array}{l}\text { Aggregation } \\
\text { interval }\end{array}$} & \multicolumn{2}{|c|}{$\begin{array}{c}5^{\text {th }} \text { harmonic current } \\
\text { prevailing phasor Site } A\end{array}$} & \multicolumn{2}{|c|}{$\begin{array}{c}5^{\text {th }} \text { harmonic current } \\
\text { prevailing phasor Site B }\end{array}$} \\
\hline & $\operatorname{Mag}[A]$ & Angle $\left[{ }^{\circ}\right]$ & $\operatorname{Mag}[A]$ & Angle $\left.I^{\circ}\right]$ \\
\hline 1-min & 2.73 & -140.15 & 3.22 & 147.68 \\
\hline 10-min & 2.73 & -140.06 & 3.22 & 147.68 \\
\hline 120-min & 2.73 & -145.63 & 3.22 & 147.67 \\
\hline
\end{tabular}

Table 7-2 list insignificant change in the prevailing phasor for the different aggregation intervals.

\subsection{Dynamic selection of aggregation time interval}

It has been shown in section 7.3 that a short aggregation time interval, such as 1-min, can yield results that show the dynamic nature of a nonlinear load such as a PV plant. A 1-min aggregation time interval may not be required when the load under investigation has a near static harmonic phase angle as shown in Figure 7-2 in the Site B. The measurement data used for a harmonic emission assessment must be representative of the non-linear load's behaviour. For a non-linear load with a high harmonic phase angle variation, a short aggregation time interval is required but for a load with a low harmonic phase angle variation a longer aggregation time interval is needed. The challenge exist when a non-linear load displays both a high and low harmonic phase variation within a given period of time. 
In this section a method is proposed and applied to dynamically change the aggregation time interval to capture the dynamic behaviour of a non-linear load with both high and low harmonic phase angle variation.

\subsubsection{Dynamic time aggregation methodology}

In most cases the default 10-min aggregation time interval is selected for PQ measurements as per IEC 61000-4-30, which may not always capture the dynamic behaviour of nonlinear loads as the interval is too long. In Chapter 5: a case has been made for the continuous assessment of harmonic emission through measurement of aggregated harmonic phasors to reduce the volume of measured data. Such a dataset can be further reduced while capturing the dynamic behaviour of nonlinear loads by dynamically changing the aggregation time interval during measurements to capture when a dynamic event occurs.

The level of prevalence, discussed in section 5.2, is an index that describe how much the phase varies of measured harmonic phasors over a time period. By constantly testing for the prevalence against a preselected value, the aggregation time interval can be dynamically selected to ensure that the dynamic nature of the nonlinear load is captured.

A method for implementing the dynamic selection of the time interval is described below. The method is based on the 10/12-cycle block values used in the IEC 61000-4-30 measurement method.

Step 1: $\quad$ User to select a level of prevalence (setpoint) to be used in the aggregation process. Selection of this value must be appropriate for the type of nonlinear load to be analysed.

Step 2: $\quad$ The initial $3000 \times 200 \mathrm{~ms}$ is measured in line with IEC 61000-4-30 principles. The $3000 \times 200 \mathrm{~ms}$ data points are equivalent to a 10 -min data sample, which is used to initially calculate the level of prevalence. The calculated level of prevalence from the measured data is tested against the preselected level of prevalence that is used as a set point.

Step 3: If the result is higher than the preselected setpoint value then the 10-min aggregated value is reported. If the result is less, the number of samples and time interval are reduced in steps as listed below, until the level of prevalence is higher than the preselected setpoint value.

1. $1500 \times 200 \mathrm{~ms}$ for 5 -min,

2. $300 \times 200 \mathrm{~ms}$ for 1 -min,

3. $5 \times 200 \mathrm{~ms}$ for 1 -sec

4. $200 \mathrm{~ms}$ (10/12 cycle value)

Step 4: If at any time the level of prevalence is higher than the preselected setpoint value, the aggregation intervals are reported and the next $3000 \times 200 \mathrm{~ms}$ data sample is selected for aggregation.

Figure 7-8 shows a flow chart and Figure 7-9 shows a decision chart of the dynamic aggregation method. 


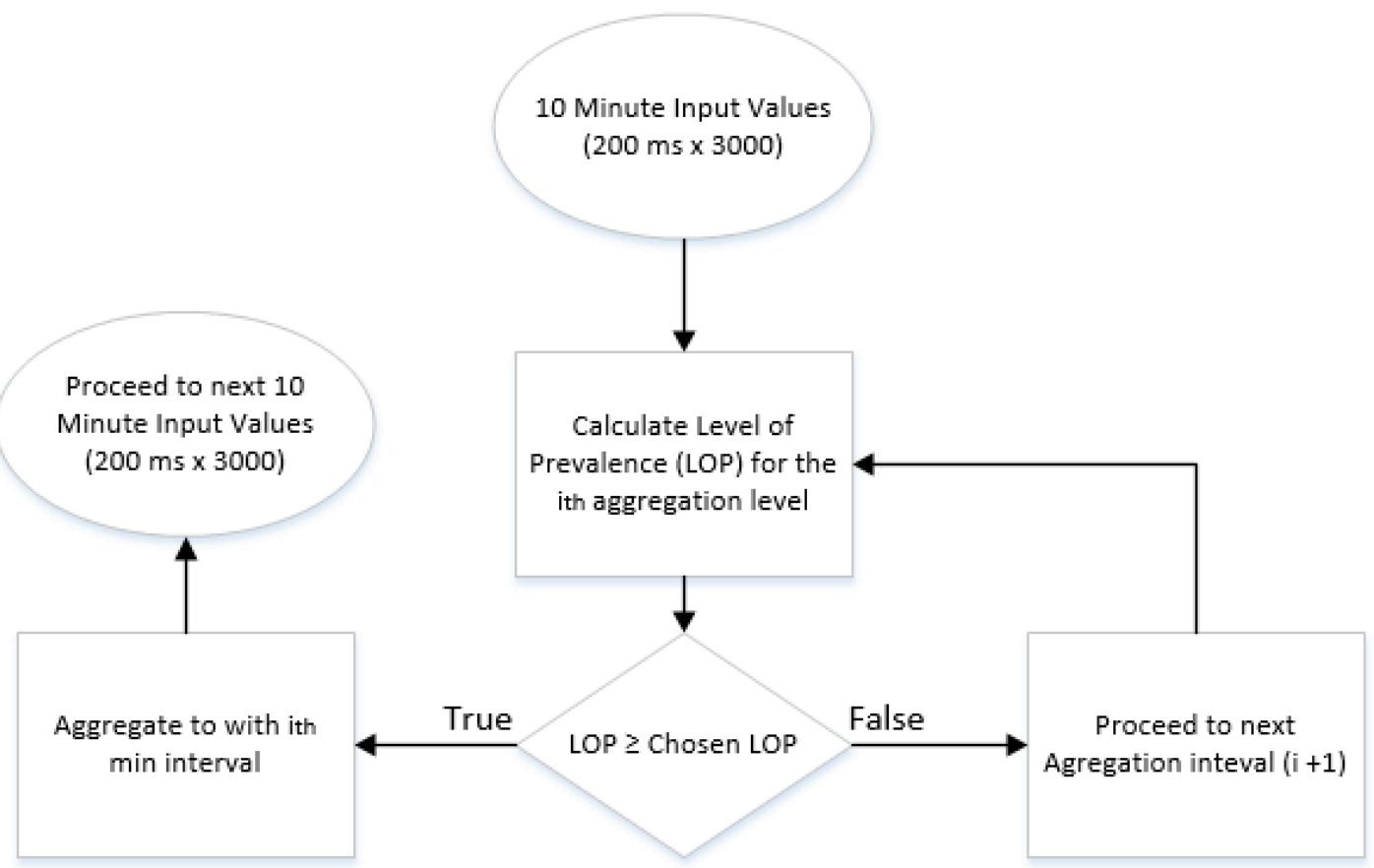

Figure 7-8. Flow chart for the dynamic aggregation method.

Aggregation Level 1

10 Minute aggregation

(200ms x 3000)

Aggregation Level 2

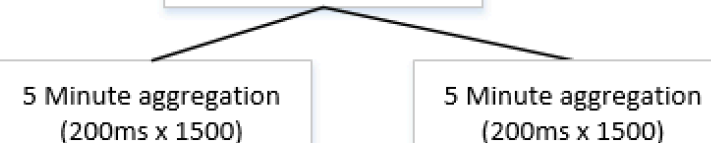

Aggregation Level 3 (200ms $\times 1500)$

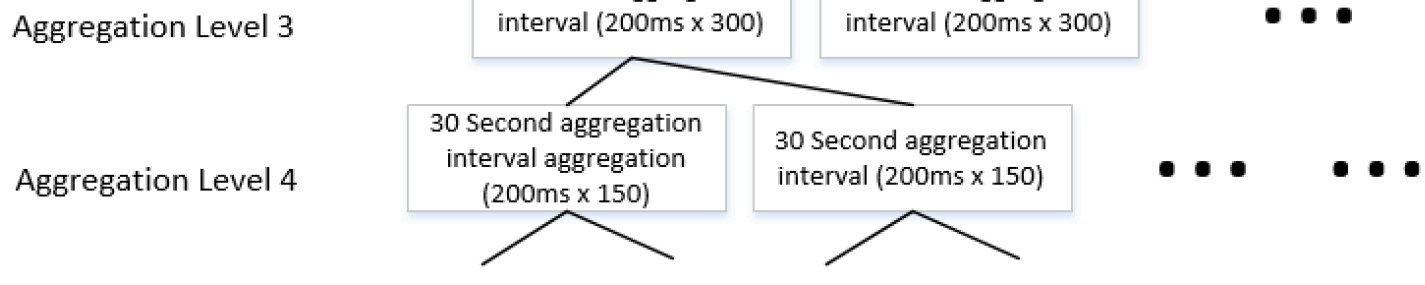

Aggregation Level ...

Figure 7-9. Aggregation levels to be used in the dynamic aggregation method.

\subsubsection{Field application of the dynamic aggregation method.}

The initial measurements used in the previous sections of this chapter did not contain any $200 \mathrm{~ms}$ data points, as such it could not be used for the application to the dynamic aggregation method.

A new measurement campaign was undertaken at an arc furnace connected to a $132 \mathrm{kV}$ network. $200 \mathrm{~ms}$ (10/12-cycle) harmonic phasor data were recorded by a PQ instrument with the aggregated phasor algorithm employed. This site was selected as variable harmonic phasor measurements is expected due to the operation of the arc furnace load. 
The following setpoint (level of prevalence) values were selected to evaluate the dynamic aggregation method; 0.9, 0.8 and 0.6. As stated before, levels of prevalence below 0.8 should not be reported but in this case 0.6 is used to test the method and to detail the possible reduction in the dataset. Note that the aim is not to evaluate the harmonic performance but rather the measurement/aggregation model performance.

Figure 7-10 shows the normal fixed aggregation time interval for the $5^{\text {th }}$ harmonic current phase angle. The figure contains the $200 \mathrm{~ms}$ plot in the top chart, the middle plot contains the 1-min aggregation and the bottom plot shows the 10-min aggregation. 120-min aggregation was not considered in this application due to the varying nature of the nonlinear load. The presented data in Figure 7-10 contains 708649 × $200 \mathrm{~ms}, 2362 \times 1$-min and 236 × 10-min data points. The measurement period presented is for less than 48 hours.
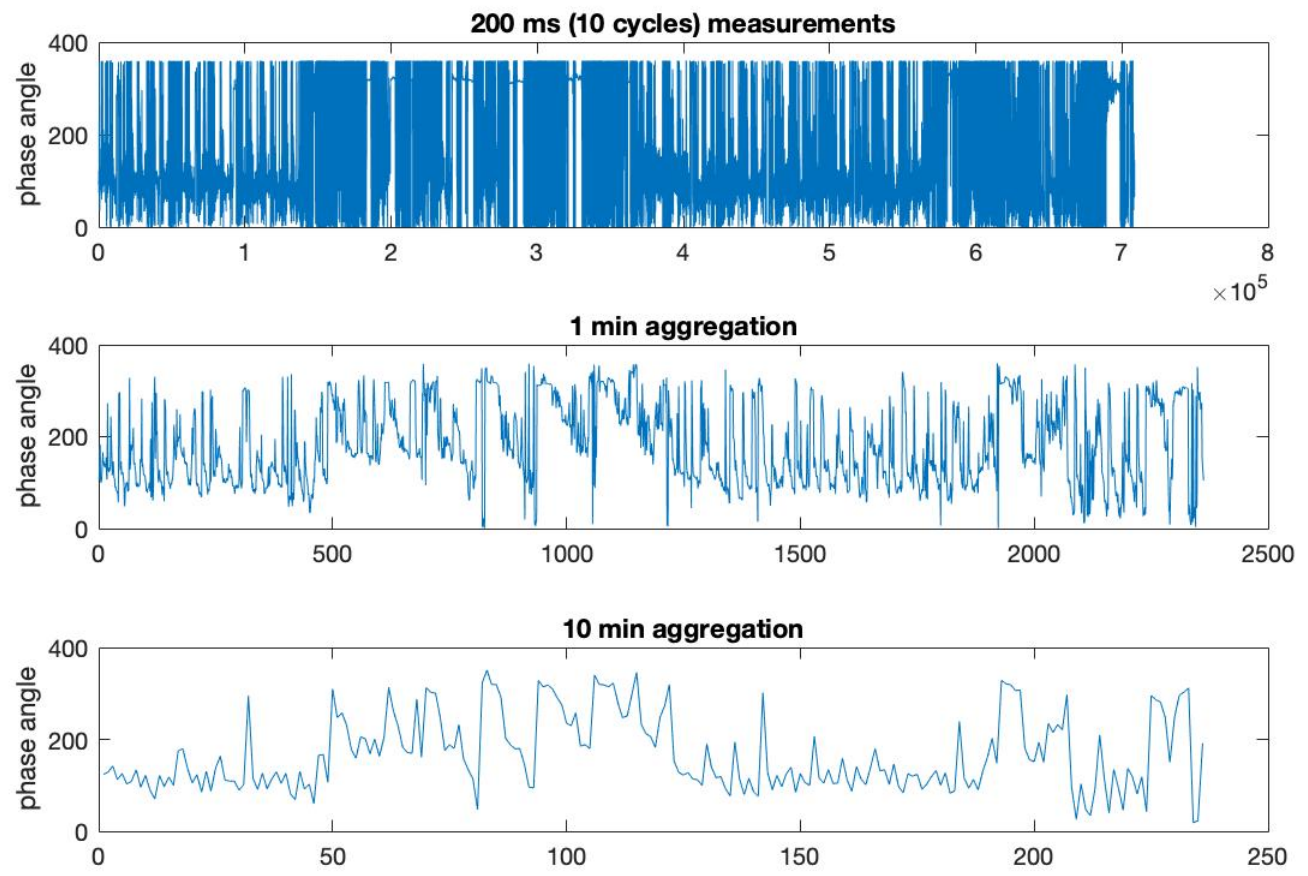

Figure 7-10. Normal aggregation of the $5^{\text {th }}$ harmonic current phase angle

Figure $7-11$ to Figure $7-13$ shows the $5^{\text {th }}$ harmonic current dynamic aggregation results for the preselected levels of prevalence.

Four plots are shown per figure in Figure 7-11 to Figure 7-13. The first chart (from the top) shows the $5^{\text {th }}$ harmonic current magnitude, the second chart show the corresponding $5^{\text {th }}$ harmonic current phase angle, the third chart charts the level of prevalence test results per aggregation time interval, the fourth chart show the reported dynamic aggregation time interval which correspond to the tested level of prevalence.

Recall that a high level of prevalence indicate that the harmonic phasor is stable with little variation during the measurement time period and that a low level of prevalence indicates a large variation in the angle of the harmonic phasor.

Figure 7-11 shows the dynamic aggregation results for the 0.9 level of prevalence. The dynamic aggregation method kept the level of prevalence above 0.9 by dynamically altering the time aggregation period as per design. In this case, a large portion of the aggregation time intervals was based on the $200 \mathrm{~ms}$ interval as listed in Table 7-3. This is due to the large variation in the harmonic 
phasors due to the arc furnace operation [107]. Harmonic current charts show that there is a reduced (below 2 A) current output, which is when the arc furnace was not in operation. As the furnace is not in operation the measured harmonic phasors should be discarded as invalid but for the purpose of the dynamic time aggregation method, the data is retained and processed.

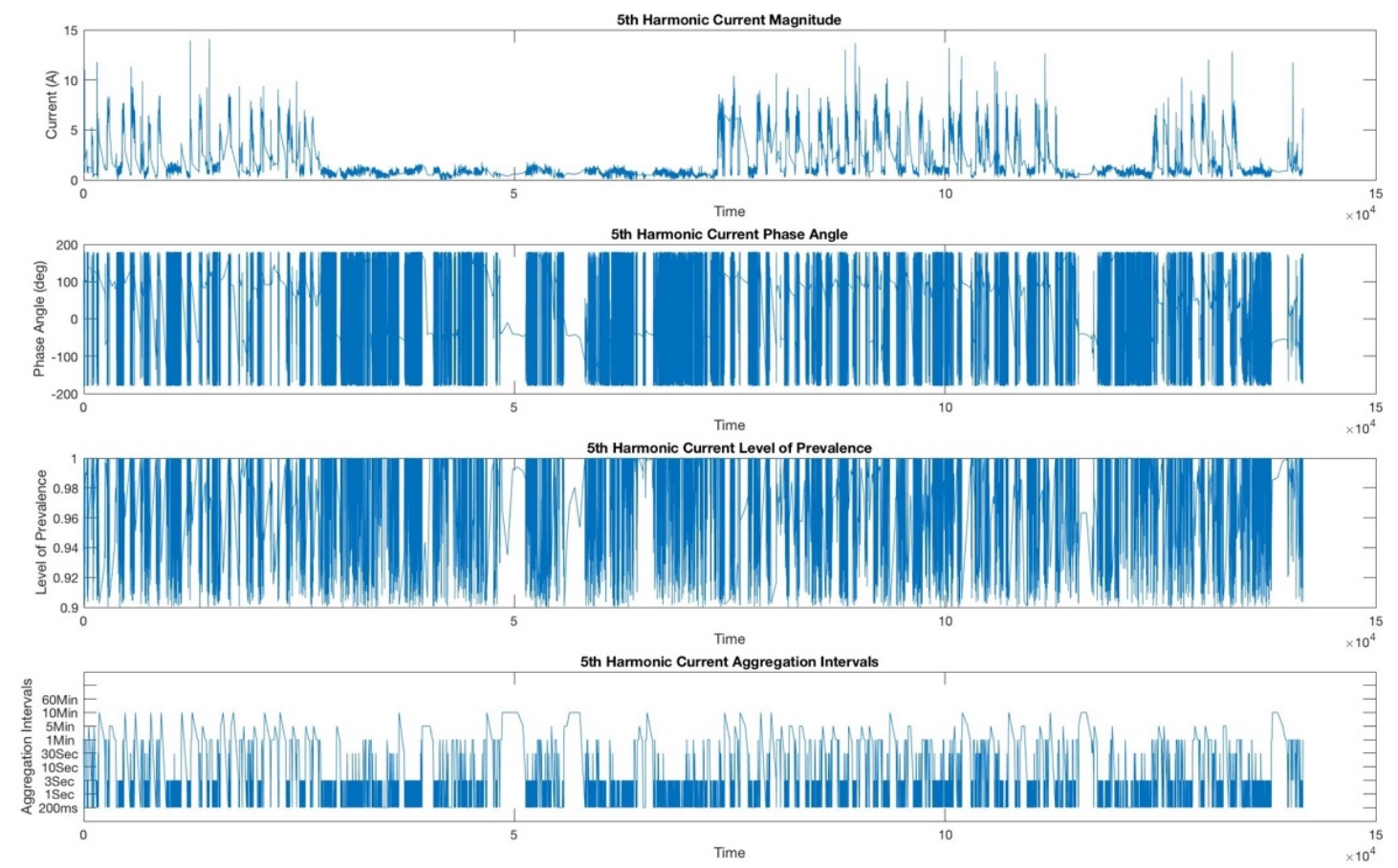

Figure 7-11. Dynamic aggregation for 0.9 level of prevalence

Figure 7-12 shows the results for the 0.8 level of prevalence. As previously indicated [73], [80] a prevalence level of 0.8 shows sufficient stability of the harmonic phasor for reporting. A small degree of variability is allowed with this prevalence level.

Observe that the level of prevalence is kept above 0.8 dynamically and a reduction in the resolution of the harmonic current magnitude and the harmonic phase angle measurements when compared to the 0.9 level of prevalence charts.

Reduction in the dataset is expected as more variability in the harmonic phasor is allowed with the 0.8 level of prevalence. 

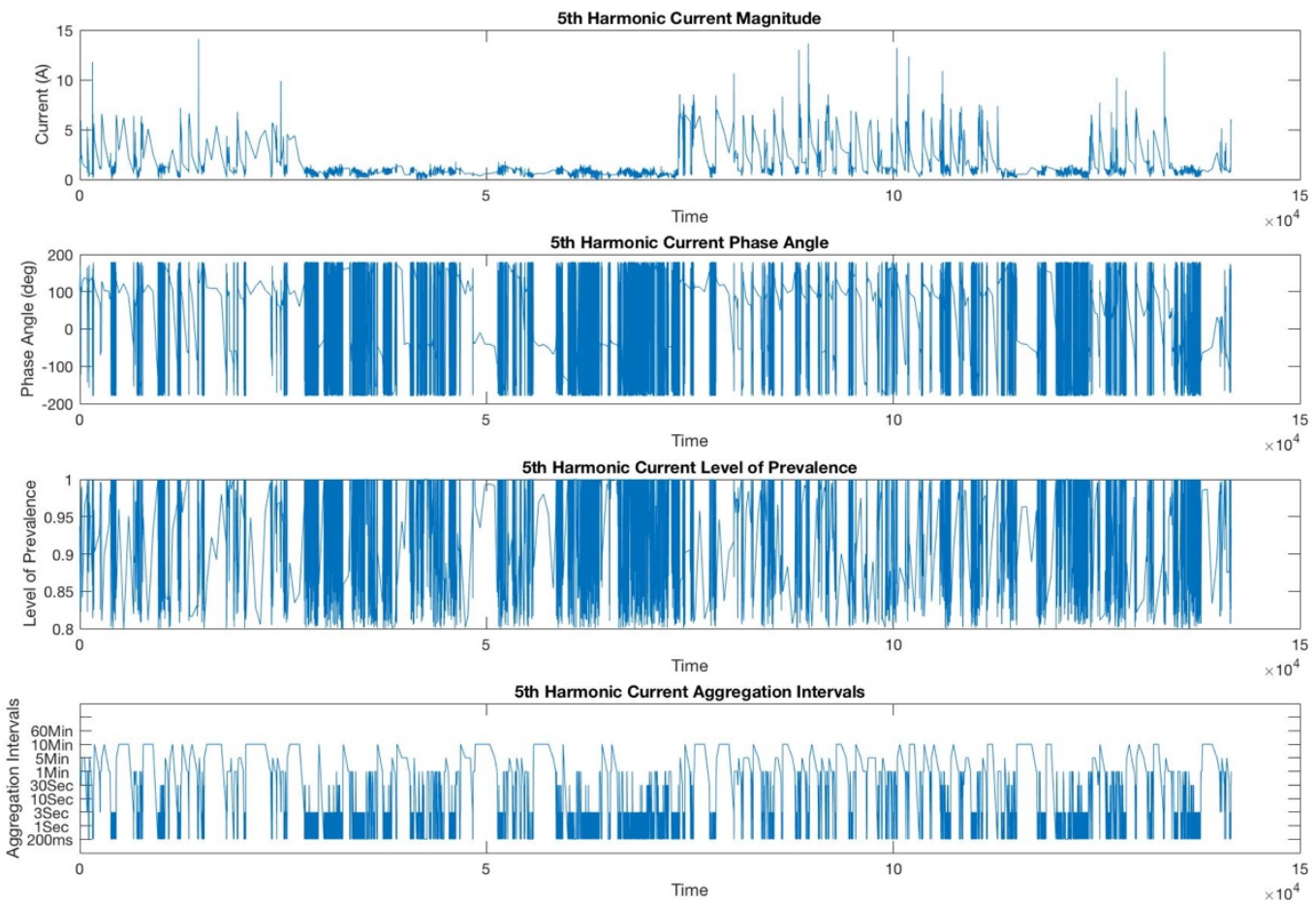

Figure 7-12. Dynamic aggregation for 0.8 level of prevalence
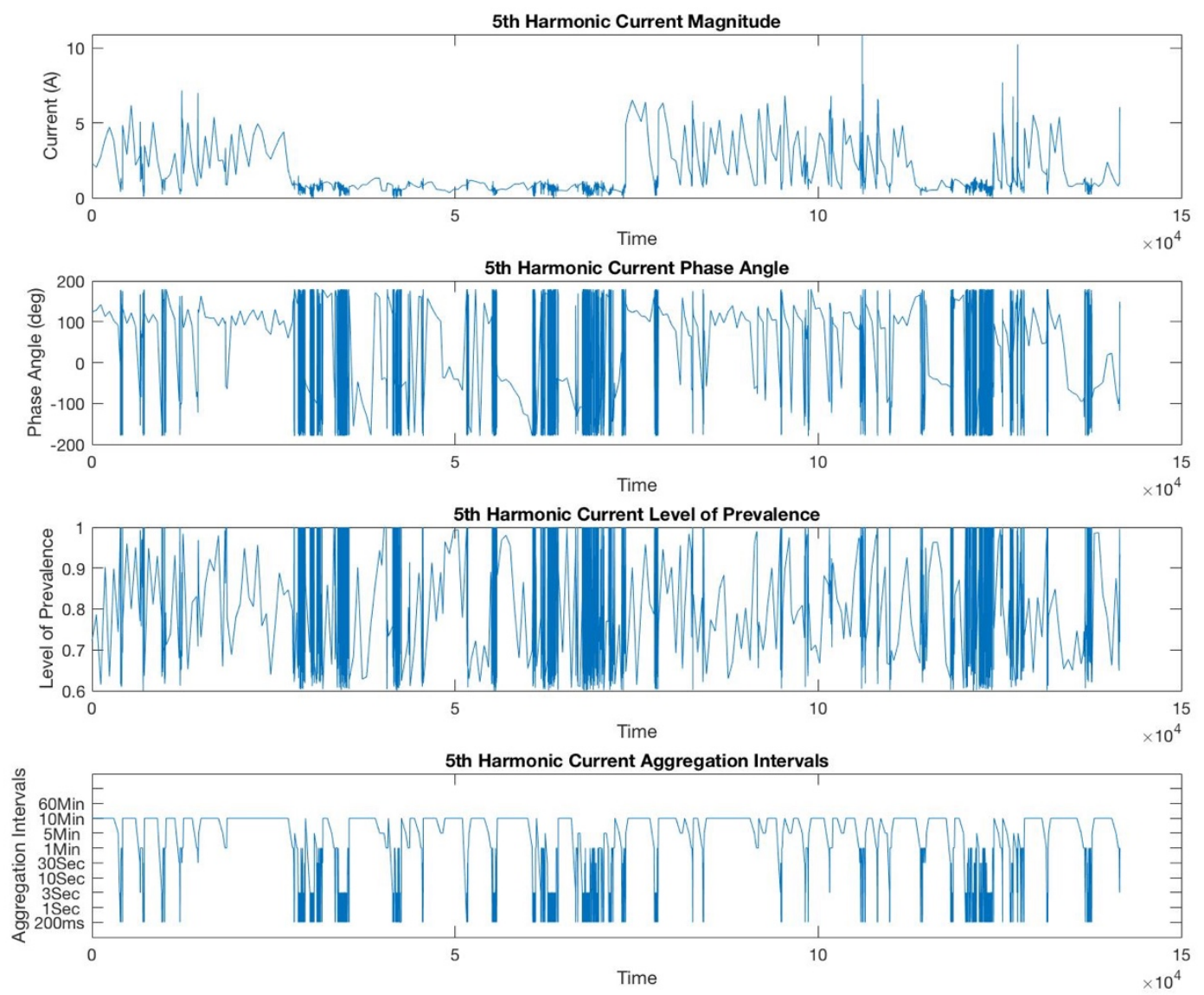

Figure 7-13. Dynamic aggregation for 0.6 level of prevalence

Figure 7-13 shows the results for the 0.6 level of prevalence. Reduction in the resolution of the presented data is observed, due to the allowed increased variability of the harmonic phasor. 
Observe that the level of prevalence was kept above 0.6. The change in the aggregation time intervals shifted to concentrate more in the 10-min range.

Table 7-3. Number of aggregation time interval points for the selected levels of prevalence.

\begin{tabular}{|c|c|c|c|}
\hline \multirow{2}{*}{$\begin{array}{c}\text { Aggregation time } \\
\text { interval }\end{array}$} & \multicolumn{3}{|c|}{ Level of Prevalence } \\
\cline { 2 - 4 } & $\mathbf{0 . 6}$ & $\mathbf{0 . 8}$ & $\mathbf{0 . 9}$ \\
\hline $200 \mathrm{~ms}$ & 37095 & 143265 & 254340 \\
\hline $3-\mathrm{sec}$ & 2367 & 5549 & 7634 \\
\hline $30 \mathrm{sec}$ & 114 & 254 & 277 \\
\hline 1-min & 231 & 393 & 392 \\
\hline $5-\mathrm{min}$ & 52 & 67 & 54 \\
\hline 10-min & 157 & 75 & 33 \\
\hline TOTAL: & & & \\
\hline
\end{tabular}

Table 7-3 list the number of data points reported based on the preselected level of prevalence. When little angular variation in the harmonic phasor is allowed, a high number of $200 \mathrm{~ms}$ data aggregation interval points are selected as evident by the 0.9 prevalence level results. The number of data points is still a fraction of the full $200 \mathrm{~ms}$ data points (708649 x $200 \mathrm{~ms}$ as in Figure 7-10) making this method viable for both the aggregation of data and representative of the dynamic behaviour of the harmonic phase angle.

As the level of prevalence is lowered the number of short $(200 \mathrm{~ms})$ interval data points are reduced and the longer aggregation time intervals are increased. Observe the decrease in the $200 \mathrm{~ms}$ data points when going from 0.9 to 0.6 prevalence level and the increase in the 10-min aggregation time intervals.

\subsection{Conclusion}

As with the uncertainty in measurements, the aggregation time interval is another measurement aspect in the assessment of harmonic emission that must be taken into consideration to ensure that harmonic emission is both fair and accurate.

With grid code assessment being absolute (no grey areas allowed) and potential financial impacts when incorrect harmonic emission assessments are undertaken, the harmonic measurement for harmonic emission assessment must reflect the dynamic nature of the nonlinear load, for example, a PV farm.

The impact of various fixed aggregation time intervals on aggregated harmonic phase angles were evaluated against two high voltage sites with different network topologies and mix of load types. The one site was a PV plant that was undergoing grid code compliance and the other a $132 \mathrm{kV}$ feeder supplying a large reticulation network with small industrial and farming loads.

\section{1-min aggregation time interval}

A fixed aggregation time interval of 1-min retains sufficient information to highlight the dynamic behaviour of a PV plant. This information is however suppressed as the aggregation time interval is increased as shown in the case study for the PV plant, where changes in the harmonic emission profile was evident in the 1-min aggregation interval and not in the 120-min aggregation interval.

Site B reported a stable harmonic phase angle throughout the measurement campaign, with high resolution measurements seen at the 1-min time interval. 


\section{0-min aggregation time interval}

The 10-min aggregation time interval is mainly used by electrical utilities to benchmark their performance and for ease of reference to regulatory documents such as EN 50160 and NRS 048.

In the field application presented, this time interval still retains information that may be used for harmonic emission assessment, as some of the dynamic behaviour information is retained.

\section{0-min aggregation time interval}

The 120-min aggregation time interval is useful when the harmonic phase angle remains "static" within a very limited range, as in the case for site B discussed in this chapter. This aggregation time interval should not be used on loads with high variability of the harmonic phasors.

\section{Dynamic selection of aggregation time interval}

The ability to dynamically change the aggregation time interval to capture sudden changes in harmonic phasors will ensure that the harmonic interaction between nonlinear loads and the supply network is adequately and fairly assessed. In the case of a PV farm, the impact of cloud cover can be captured as harmonic emission during this period changes.

Online testing for the level of prevalence against a setpoint level of prevalence is used to evaluate which aggregation time interval is be used for the harmonic measurements during dynamic conditions. Application of this novel dynamic aggregation method to $200 \mathrm{~ms}$ harmonic data, has shown how time aggregation intervals were altered for different levels of prevalence and how a dataset can be significantly reduced while still reporting dynamic behaviour of nonlinear loads.

Grid code compliance is achieved with a fixed measurement period of 7 days and using 10 min aggregated rms values. It was shown in this thesis that the use of an rms value is not adequate and, in this chapter, it is shown that selection of the aggregation time interval may influence the quality of the measurement dataset. It is recommended that harmonic emission assessment at renewable power plants be done on a continuous basis and not a once off exercise as the harmonic emission of a renewable power plant can change over time and depending on the network it is connected into. Through the application of the dynamic aggregation method the duration of measurements can be increased significantly while still retaining a reduced dataset reflective of the harmonic emission profile of a non-linear load.

In conclusion, the use of fixed aggregation time intervals is a common practice with electrical utilities aiming to report on the PQ performance with 10-mins being selected as the default value. When evaluating the impact of a nonlinear load or in conducting a harmonic emission assessment, a fixed aggregation time interval may not sufficiently report the harmonic phasor. 1-min aggregation values are commonly used in literature and does report sufficient content for harmonic emission assessment. 120-min values should not be considered for harmonic emission. Application of the new dynamic aggregation method ensures that the measured phase angle data is meaningful, while reducing the reported data as well as capturing dynamic events that could impact a harmonic emission assessment. 


\section{CHAPTER 8: Conclusion}

\subsection{Introduction}

The purpose of this chapter is to present the key findings of the thesis. Results obtained during the project are tested against the hypotheses made. The contributions made are discussed in section 8.4 and the chapter is concluded with recommendations for future work.

\subsection{The need for a discriminative approach to harmonic emission assessment}

Harmonic emission assessment has been dealt with extensively in literature with various approaches to the measurement methods and techniques on how the harmonic emissions must be assessed. However, the methods presented are either not pragmatic or conclusive - hence the need for the research documented in this thesis.

Renewable Power Plants (RPP) undergoing grid code compliance requires a fair and pragmatic approach. RPPs should not be held liable for harmonic emissions which are being absorbed by their plant, i.e. aiding the electrical utility. With 10-min aggregated rms measurements as prescribed by the South African grid code, discrimination of values being absorbed and those being emitted is not possible without phase angle data or expansive measurement campaigns.

The debate on who is accountable for the harmonic distortion at the point of common coupling between the load/RPP and the utility has not been scientifically solved, within the constraint of a 10-min rms value. Using the 10-min rms values at best a qualitative approach [19] as to who is the major contributor towards the harmonic distortion can be undertaken.

Grid code compliance for RPPs is absolute with no grey areas allowed, which is why a discriminative approach to harmonic emission assessment is required to ensure that only harmonic distortion being emitted by the RPP is used for the compliance assessment. Accounting for all of the harmonic emissions is not fair and may lead to unnecessary financial expenditure on mitigation equipment by a RPP.

The majority of the harmonic emission methods and techniques, investigated in this thesis, make use of measured phasor data to evaluate the harmonic emission from a nonlinear load, but the challenge with "pure" phasor data is the large volume of data that result when measurements are taken for more than one day.

Aggregation of data (via IEC 61000-4-30 principles) is a common practice in reducing the measured data set to a manageable volume of data and measurements can also be taken over a much longer time period, over days for example. Only rms data results from the aggregation process, rendering the assessment of harmonic emission direction a problem that electrical utility engineers constantly face.

\subsection{Assessing the hypothesis}

From Chapter 1: it is hypothesised that:

Grid code compliance requirements on harmonic emission can be assessed in a fair and pragmatic manner by means of an improved method recognising that:

- A nonlinear and dynamic interaction between the supply network and the grid-connected RPP result in harmonic currents being injected or absorbed by either.

- Assessment of harmonic emission has to identify those harmonics being injected into the supply network and has to exclude those being absorbed by the RPP. 
- Harmonic emission assessment has to be done continuously.

The hypothesis is now discussed in terms of the following research questions and statements:

\subsubsection{Analysis and modelling of harmonic emission from first principles in an interconnected network with more than one source of harmonic waveform distortion are reported.}

In Chapter 3:, simulations were undertaken to demonstrate the concept of harmonic emission based on a simple Thévenin and Norton equivalent circuit. Various harmonic emission assessment methods were successfully applied to this simple circuit to verify these methods from first principles. These simulations were however based on a single nonlinear load application. To demonstrate the harmonic distortion impact of multiple nonlinear loads connected to the same node, a simulation model was built with two identical thyristor controlled inverters.

Simulation results from the thyristor controlled nonlinear loads show that harmonic emission is a real concern and that the assessment of harmonic emission is not a simple task, but rather a much more complex than is suggested by the general summation law as given in IEC 61000-3-6. The simulation confirmed the need for harmonic phasors to correctly assess harmonic emission. Using rms values in isolation will lead to incorrect analysis and unnecessary expenditure in mitigation equipment.

Analysis further shows that harmonic interaction between the two nonlinear loads not only impact the harmonic emission from each other, but also impact the upstream network harmonic distortion such that in some conditions the harmonic distortion levels on the upstream network were reduced. This is a key finding in support of a discriminatory approach to harmonic emission assessment. Non-linear loads such as RPPs should only account for the harmonic emission that they emit or in which they help the upstream supply network through their operations.

\subsubsection{The metrological requirements and constraints of measuring both voltage and current harmonics are derived.}

Two key aspects affect the measurement of voltage and current harmonics, namely the instrument transformers (transducers) and overall measurement uncertainty.

Studies [83]-[86] have shown that instrument transformers play a role in the accuracy of the harmonic measurements.

\section{Instrument transformers}

The frequency response of instrument transformers is not linear. Voltage transformers tend to be accurate below $1 \mathrm{kHz}$ [51], however, higher frequencies require characterisation of the frequency response for the voltage transformer being used. Different types of voltage transformers have different frequency responses, with a capacitive voltage transformer not being recommended for harmonic voltage measurements.

Current transformers have a higher frequency range in which harmonic distortion can be measured. Accurate harmonic measurements are possible up to $10 \mathrm{kHz}$ [87].

\section{Uncertainty in measurements}

Uncertainty in measurement is not just a factor of the instrument transformers being utilised, but also the remaining equipment that makes up the measurement circuit or chain. Harmonic response of instrument transformers and the measurement circuit must be characterised to accurately evaluate the harmonic emission from a non-linear load. Unfortunately, it is not always practical to characterise instrument transformers when they are installed and in use, reliance is placed on 
documents such as IEC 61869-103 to obtain the uncertainty values that can be used in an uncertainty budget calculation.

The Guide for Uncertainty in Measurement (GUM) provides a statistical approach to qualifying measurement uncertainty by making reasonable assumptions and using nameplate data. In more accurate studies [96] the frequency response of instruments are characterised and then applied to the uncertainty budget. Application of GUM to the measurements utilised in this document added confidence to the harmonic current measurements. Quantifying the measurement uncertainty can prevent an RPP from fruitless expenditure on harmonic mitigation equipment when their assessed harmonic emissions values exceed the allowable limits set by the utility.

In this thesis the measurement uncertainty was applied to a RPP that is undergoing grid code compliance and an uncertainty band was established around the harmonic emission value. The uncertainty band highlighted a possible point of conflict where the RPP will use the lower value of the uncertainty band and the DSO will use the upper value of the band. RPPs should be given the benefit of the doubt (which is uncertainty) especially when the DSO can accommodate the minor exceedance on their emission limits.

The metrological aspects of harmonic emission assessment must be taken into account to fairly evaluate harmonic emission in order to avoid disputes.

\subsubsection{Can the relative contribution to harmonic distortion be quantified based on rms harmonic measurements obtained from IEC 61000-4-30 Class A edition 3 compliant instrumentation?}

Electrical utilities that have power quality programs which report their performance against Power Quality (PQ) performance management documents such as EN 50160 and NRS 048 by default measure only aggregated 10-min voltage rms values according to IEC 61000-4-30.

The South African grid code on renewable energy integration calls for the measurement of 10-min aggregated values according to IEC 61000-4-30 Class A in the grid code PQ compliance procedure.

CIGRE/CIRED working group C4.109 developed a method to evaluate who the major contributor is towards the harmonic distortion at a PoC by only measuring the 10-min rms harmonic voltage and harmonic current at the Point of Connection (PoC). The qualitative analysis of the CIGRE method is through the use of a scatterplot containing the measured harmonic current and voltages as well as the respective harmonic impedance slopes of the RPP and the upstream network at a specific harmonic order. Evaluation criteria for this method contains three scenarios,

- when the load is the major contributor,

- when the network is the major contributor,

- and when they both contribute towards the harmonic distortion at the PoC.

With the constraint of a 10-min aggregated rms value, the CIGRE/CIRED C4.109 method was applied to a PV plant undergoing grid code compliance. Using measurements at a single point (typically the PoC) the deficiencies in the method was highlighted as follows;

- There is no discrimination between harmonics being emitted or absorbed

- Lack of qualitative interpretation of scatterplots results for points that do not follow the three scenarios as prescribed.

Application of a multiple-point measurement system, that is GPS synchronised to ensure that the measurements are coherent in each time stamping, was used to discriminate harmonic distortion which are emitted and those being absorbed by the RPP. The method was used to quantify the 
harmonic emission from a single nonlinear load. Results were qualitatively verified by an alternative method to ensure that discrimination was achieved.

\subsubsection{Can existing harmonic assessment techniques be improved to sufficiently discriminate between harmonic emission contributed by different Renewable Power Plants (RPP) or by a specific distorting load, or is a completely new approach required?}

A harmonic emission assessment should only take into account the harmonic distortion which is emitted according to the IEC 61000-3-6 definition of harmonic emission. In this thesis harmonic emission assessment methods and techniques are evaluated in an attempt to discriminate between harmonic emission which is absorbed/sunk by the RPP and the emission emitted/generated by the RPP. Findings from the applied methods and techniques are discussed below.

\section{CIGRE/CIRED C4.109}

Application of the CIGRE/CIRED C4.109 method to RPPs found that rms single-point measurement alone cannot discriminate between harmonic distortion being emitted against those being absorbed by an RPP or other nonlinear loads. Furthermore, the three listed qualitative interpretations of the scatter plots do not cater for cases that deviate from these mentioned norms. It was shown that the $7^{\text {th }}$ harmonic order for one of the PV plants under evaluation in this thesis showed inconclusive results as the interpretation of the scatterplot did not confirm to the listed means of interpretation. The lack in interpretation of the scatter plots poses a challenge to RPPs attempting to prove their compliance as their harmonic emission values cannot be evaluated using this method and it often leads to disputes between the DSO and the RPP.

The CIGRE method was improved through the application of GPS synchronised multiple point measurements and it was shown that this improvement can remove those harmonic measurements which are not deemed as emission. The interpretation of the discriminated scatter plots remained a challenge in this qualitative method.

Although the method was improved to include discrimination, the resultant harmonic emission values between discriminated and non-discriminated values remained almost the same. This is due to the use of a $95^{\text {th }}$ percentile value as the harmonic emission. When the discriminated values were removed it did not remove any values above or close to the $95^{\text {th }}$ percentile value of the measurement data set, hence the results remained the same.

\section{Prevailing phase angle}

As indicated in Chapter 2 harmonic emission assessment requires the measurement of phasors, but a large volume of data results when "pure" phasors are measured. The prevailing phase angle is a method whereby harmonic phase angle information is retained after the Fourier analysis of the 10/12-cycle measurements which are aggregated in line with IEC 61000-4-30 principles. Both aggregated harmonic rms and phase angle information can be reported over much longer periods without the large volume of data that results from cycle by cycle phasor measurements, depending on the aggregation time interval selected.

Measurement of the previously mentioned PV plant was revisited but with 1-min aggregated prevailing phasor measurements being taken in conjunction with the 10 -min rms values. Analysis of the measurements using the prevailing phase angle method confirmed the results of the multiplepoint measurements where discrimination was applied. With the phase angle information now at hand, clarity on the inconclusive assessment of the $7^{\text {th }}$ harmonic (using CIGRE/CIRED C4.109 method) could be confirmed.

Discrimination of harmonic emission was achieved by application of the Range of Secondary Cancellation (RSC) technique where an angular range is calculated per connection point for each 
harmonic order. This angular range will highlight if a harmonic phasor, measured at a non-linear load, will have a positive or negative impact on the harmonic voltage at the PoC. Other possible applications of the RSC technique were also evaluated where the performance of harmonic filters banks were investigated. In the field study it was shown the one filter bank added no value while other filter banks operated as per design for the site that was investigated.

A pragmatic engineer can conclusively conduct an harmonic emission assessment at a RPP by measuring the aggregated harmonic phasors, which is easily implemented into a modern PQ recorder, and applying the RSC technique to the measured harmonic current in determining which values can be deemed harmonic emission and which cannot. The RSC method requires only knowledge of the network harmonic impedance at the $\mathrm{PoC}$ for it to be implemented and measurements can be taken at a single point as opposed to expansive measurement campaigns.

\section{Application at HV networks}

The prevailing phase angle method was applied to MV and LV networks in literature. In this thesis, the method was applied to a high voltage $(132 \mathrm{kV})$ network where multiple nonlinear loads, such as large scale PV plants and traction loads, are connected onto the same node. Application of the method highlighted the plight of RPPs attempting to prove grid code compliance in an environment where other nonlinear loads exist.

The dynamic nature of harmonic interaction at a PCC was highlighted in a multiple-point measurement campaign using the prevailing phase angle method. A comparison was done between the IEC 61000-3-6 general summation rule and the prevailing phase angle method in calculating the harmonic emission values that will be used to compare against the limits provided by the DSO. The comparison highlighted that the application of the general summation rule yields higher harmonic emission values than the prevailing phase angle method. This was due to the conservative assumptions made in the summation exponents and the IEC method did not take harmonic phase angle cancellation into account.

Discrimination was again applied to the high voltage measurements by application of the RSC method. Harmonic emission results under discriminated conditions showed a reduction in the harmonic emission at the PV plants under consideration. This is considered to be a fairer means of harmonic emission assessment as a discriminated approach only takes into account the harmonic distortion emitted from a non-linear load such as a PV plant.

\section{Harmonic emission assessment under dynamic conditions.}

A different approach is required when a harmonic emission assessment is to be conducted at a site where dynamic conditions are prevalent. It has been shown that a 10-min rms value alone cannot sufficiently qualify the dynamic behaviour or conditions of harmonic interaction between nonlinear loads and the upstream network, especially when applying the CIGRE/CIRED C4.109 statistical approach.

Application of the RSC method has shown to result in values that can be used to discriminate between harmonics which are emitted or absorbed under dynamic conditions due to the measurement of aggregated harmonic phasors.

A new method to measure these dynamic conditions was developed to report meaningful harmonic phasors by dynamically adjusting the aggregation time interval. Changes in the time aggregation period is achieved through constant testing for the level of prevalence against a preselected setpoint. If the level of prevalence is above the setpoint then the time interval is retained, the opposite is true when the level of prevalence is below the setpoint where the time interval is shortened. In altering the time interval dynamic events can be captured at higher resolution for 
improved harmonic emission assessment while still maintain data integrity and reduction of a dataset if measurements is taken over long durations.

In combining the dynamic aggregation time interval method and the prevailing phase angle method, discrimination of harmonic emission or absorption is achieved under dynamic conditions. This application can also be used when harmonic emission is done on a continuous basis as it was shown in this thesis that harmonic emission from a non-linear load is not static and changes over time.

\subsubsection{Confirmation of hypothesis}

Based on the results of the research questions listed above the hypothesis is confirmed in that;

- It was shown that harmonic emission is both absorbed and emitted by non-linear loads through both simulations under controlled conditions, and field measurements of sites with multiple non-linear loads connected onto the same node. It was further shown that the network harmonic distortion can be reduced through the harmonic interaction of these nonlinear loads, which is not easily measured by an rms value only.

- Harmonic emissions can be conducted in a fair and pragmatic manner through the application of the prevailing phase angle measurement method (aggregated harmonic phasors) and the range of secondary cancellation technique. Through these methods discrimination of harmonic emission from an RPP can be achieved to only report those values which are deemed as harmonic emission.

- A harmonic emission assessment at a PV plant using aggregated harmonic phasors has shown that the harmonic emission from this plant is not static and can change considerably from one instance to the next over a 24 hour assessment period. Aggregation of the harmonic phasors allows for a longer and continuous measurement periods in order to capture these dynamic changes in the harmonic emission profile from a PV plant, as example. The period of the harmonic emission assessment is further increased or improved by dynamically changing the aggregation time interval that reduce the time interval when there is a dynamic event as seen when the harmonic phasor changes rapidly and increases the time interval when the harmonic phasor remains relatively static.

\subsection{Contributions}

Contributions made in this thesis are towards the application and improvement of existing harmonic emission assessment methods for implementation by a pragmatic engineer as a tool for harmonic emission assessment.

- The CIGRE/CIRED C4.109 method was constrained by a 10-min rms harmonic voltage and harmonic current measurement. While the method accounted for who the major contributor was towards the harmonic distortion at the PoC, it could not discriminate between those values considered to be harmonic emission against those that could not be considered as emission. By implementing a GPS synchronised multiple measurement point system and implementing the condition that $\left|\boldsymbol{V}_{h}\right|>\left|\boldsymbol{E}_{h 0}\right|$, the CIGRE/CIRED C4.109 method was improved to achieve discrimination of harmonic distortion being absorbed and those being emitted by a non-linear load such as a PV plant.

- Largescale RPPs are constantly being integrated into high voltage systems where multiple non-linear loads are connected to the same node. Application of the prevailing phase angle measurement method to such a high voltage system highlighted the harmonic emissions compliance dilemma that RPPs are faced with when they are integrated into such a network point. Application of a rms value based assessment method will not fairly account for the 
harmonic emissions from a single PV plant only. Discrimination of harmonic emission was achieved through the measurement of aggregated harmonic phasors and applying the range of secondary cancellation technique to result in harmonic emissions from only the PV plant under investigation.

- Measurement of phase angles is not a new concept, but the aggregation of phase angles is. In literature only a fixed aggregation time interval is considered for the aggregation of harmonic phasors. Depending on the time interval selected, certain dynamic events may be omitted from the measurement dataset which will not be representative of the non-linear load behaviour. Based on the level of prevalence as a preselected setpoint, an innovative technique was developed to dynamically alter the aggregation time interval depending on the variation of the harmonic phasor. The technique was applied to an industrial site where a reduction in the measurement dataset was reported as well as the dynamic character of this industrial load. Alterations in the level of prevalence set point showed how the reduction in the dataset can be controlled while still maintaining data integrity.

- The prevailing phase angle measurement method in conjunction with the newly developed dynamic aggregation time interval will enable the continuous assessment of harmonic emission and the capture dynamic behaviour from harmonic distortion in greater detail resulting in improved harmonic emission assessments.

The research questions thus confirm the hypothesis that harmonic emission is dynamic, and that discrimination is required to ensure grid code compliance is fair and pragmatic.

\subsection{Significance of results}

The following points detail the significance of the results in this thesis.

- Grid code compliance is absolute and RPPs attempting to conduct a harmonic emission assessment with only rms values at a single point may financially be negatively impacted due to incorrect assessments. The CIGRE/CIRED C4.109 method was improved through the use of a multiple point measurement system and it was shown that discrimination of harmonic emission values was possible with a 10 -min rms value. RPPs can thus only account for their harmonic emission in a fair manner. The CIGRE/CIRED C4.109 method however still lacks the interpretational means when data sets do not conform to the prescribed interpretational guidelines.

- Confidence in the prevailing phase angle measurement method, as applied in this thesis, is evident in the publication of a new Technical Report IEC 61000-1-8 [108] document dealing with prevailing phasors at LV level. The IEC report only presents the prevailing phasors at various measurement points in various countries, whereas in this thesis the prevailing phase angle measurement method is applied to RPP's to fairly conduct harmonic emission assessments prior to the publication of the IEC document.

- Application of the prevailing phase angle at a high voltage network where multiple nonlinear loads are connected onto the same busbar, highlight the dynamic behaviour of harmonic distortion. It has been shown through simulations in this thesis that harmonic interaction between non-linear loads do not add or subtract in a fixed manner as suggested by the IEC 61000-3-6 general summation law when only rms values are considered. In the field examples analysed, it was shown that the use of the IEC 61000-3-6 general summation law yields conservative results compared to the case when aggregated harmonic phasors are used to summate the harmonic distortion from multiple non-linear loads. The use of aggregated harmonic phasors is thus an improvement on the IEC 61000-3-6 general summation law to result in correct harmonic emissions assessments. 
- The dynamic change in the aggregation time interval is a further significant contribution as the method will ensure that harmonic phasors are meaningful (i.e. comply to a certain level of prevalence) before they are reported to a measurement dataset. Continuous harmonic emission assessment is possible with this method due to the reduced dataset that results, note that the amount of reduction is dependent on the preselected level of prevalence that will be allowed. The potential to expand this method to other PQ parameters will ensure that dynamic events are captured for analysis purposes, especially when an industrial process is interrupted leading to financial impacts or damage to equipment due to a rise in voltage unbalance levels as an example.

\subsection{Recommendations for RPPs}

The South African grid code on renewable integration dictates the methodologies to be applied by an RPP to prove their grid code compliance, with no deviations allowed. The prescribed methodologies are based on 10-min rms values measured according to IEC 61000-4-30 class A edition 2.

The author of this thesis compiled several changes to the South African grid code on renewable integration which at, the date of compiling this thesis, is going through the approvals process. The changes which include;

- The measurement of aggregated harmonic phasors

- The use of harmonic emission assessment methods that make use of harmonic phasor measurements, including aggregated

- Measurement uncertainty assessment.

Once approved the new guideline document will enable RPPs to conduct harmonic emissions assessment based on the methods discussed in this thesis.

It is recommended that RPPs make use of the new methods in this thesis as they have already agreed to the methods as part of the grid code approval process.

\subsection{Future work}

It has been shown in this document that the aggregation of harmonic phasors by means of the prevailing phase angle method is a tool that a pragmatic engineer can use to evaluate harmonic emissions.

During the research work several further works emerged as discussed below.

\subsubsection{Data clustering}

The polar plots used in this thesis made use of heat plots to showcase where a harmonic phasor typically operates. In some cases, heat clouds could not be observed due to the harmonic phasors operating in different quadrants and operating conditions of the non-linear load.

The following research question is asked;

- Based on aggregated harmonic phasor measurements, can data clustering practically be used to discriminate between harmonic distortion absorbed against those being emitted by a non-linear load? 


\subsubsection{Complex harmonic active power in the presence of multiple non-linear loads}

Non-linear loads are constantly being integrated onto high voltage busbars with multiple nonlinear loads. Literature [16] indicate that harmonic active power is useless when more than one harmonic source exist at the point of connection.

- Can complex active power be used to discriminate harmonic emission from a single load that is connected into point of connection with multiple non-linear loads on the same node?

\subsubsection{Dynamic aggregation time interval}

In this thesis the aggregation time interval for harmonic measurements is dynamically changed to firstly report events with the highest allowable resolution and secondly to reduce the measurement dataset. The allowable resolution is based on the level of prevalence that is preselected. Further research into the preselection of the level of prevalence

- What is the optimal preselected level of prevalence for a measurement dataset to be reflective of the non-linear load that is being investigated? 


\section{BIBLIOGRAPHY}

[1] NERSA, "GRID CONNECTION CODE FOR RENEWABLE POWER PLANTS (RPPs) CONNECTED TO THE ELECTRICITY TRANSMISSION SYSTEM (TS) OR THE DISTRIBUTION SYSTEM (DS) IN SOUTH AFRICA,” 2019.

[2] D. of Energy, "Independent Power Producer Procurement Programme." [Online]. Available: https://www.ipp-projects.co.za/. [Accessed: 22-Aug-2018].

[3] NERSA, "The South African Grid Code Governance Code," 2014.

[4] IEC, "Electromagnetic compatibility (EMC) - Part 3-6: Limits - Assessment of emission limits for the connection of distorting installations to MV, HV and EHV power systems," Geneva, Switzerland, 2008.

[5] M. Farhoodnea, A. Mohamed, H. Shareef, and H. Zayandehroodi, "Power Quality Impact of Renewable Energy based Generators and Electric Vehicles on Distribution Systems," Procedia Technol. 4th Int. Conf. Electr. Eng. Informatics, vol. 11, no. Iceei, pp. 11-17, 2013.

[6] V. Ciucur, "Power quality analysis of renewable energy sources in Romania," 2014 16th Int. Conf. Harmon. Qual. Power, pp. 699-702, 2014.

[7] NRS, "NRS 048 ELECTRICITY SUPPLY - QUALITY OF SUPPLY PART 2: VOLTAGE CHARACTERISTICS , COMPATIBILITY LEVELS , LIMITS AND This document is not a South African National Standard," 2015.

[8] S. Bhattacharyya, S. Cobben, P. Ribeiro, and W. Kling, "Harmonic emission limits and responsibilities at a point of connection," IET Gener. Transm. Distrib., vol. 6, no. May 2011, p. 256, 2012.

[9] M. G. Ippolito, G. Morana, and F. Russo, "A contribution to solve the problem of attributing harmonic distortion responsibility," in 18th International Conference on Electricity Distribution, 2005, no. 2, pp. 6-9.

[10] G. V. De Andrade, S. R. Naidu, M. G. G. Neri, and E. G. Costa, "Estimation of the Utility ' $\mathrm{s}$ and Consumer' s Contribution to Harmonic Distortion," IEEE Trans. Instrum. Meas., vol. 58, no. 11, pp. 3817-3823, 2009.

[11] F. H. Costa, I. N. Santos, S. F. P. Silva, and J. C. De Oliveira, "A Case Study of Sharing the Harmonic Voltage Distortion Responsibility between the Utility and the Consumer," in International Conference on Renewable Energies and Power Quality (ICREPQ'09), 2009.

[12] P. S. Chandramohanan Nair and F. M. Fernandez, "Method for separation of customer and utility contributions of harmonics at point of common coupling," IET Gener. Transm. Distrib., vol. 7, no. October 2012, pp. 374-381, 2013.

[13] T. Pfajfar, B. Blažič, and I. Papič, "Harmonic Contributions Evaluation With the Harmonic Current Vector Method," IEEE Trans. POWER Deliv., vol. 23, no. 1, 2008.

[14] M. Farhoodnea, A. Mohamed, H. Shareef, and R. A. J. Khan, “An Improved Method for Determining Contribution of Utility and Customer Harmonic Distortions In A Power Distribution System," Int. J. Electr. Eng. Informatics, vol. 2, no. 3, 2010.

[15] M. Bazina and T. Tomiša, "Comparison of various methods for determining direction of harmonic distortion by measuring in point of common coupling," in ENERGYCON 2014 IEEE International Energy Conference, 2014, pp. 392-399. 
[16] P. H. Swart, J. D. van Wyk, and M. J. Case, "On techniques for localization of sources producing distortion in three-phase networks," Eur. Trans. Electr. Power, vol. 6, no. 6, pp. 391-396, 2007.

[17] A. P. J. Rens, "Validation of popular nonsinusoidal power theories for the analysis and management of modern power systems," North West University, 2005.

[18] A. P. J. Rens and P. H. Swart, "On Techniques for the Localisation of Multiple Distortion Sources in Three-Phase Networks: Time Domain Verification," Eur. Trans. Electr. Power, vol. 11, no. 5, pp. 317-322, 2001.

[19] E. DE Jaeger, "Disturbance Emission Level Assessment Techniques ( CIGRE / CIRED Joint Working Group C4-109)," in 20th International Conference on Electricity Distribution, 2009, no. 0470, pp. 8-11.

[20] B. B. Peterson, J. Rens, M. G. Botha, and J. Desmet, "On Harmonic Emission Assessment : A Discriminative Approach,” Africa Res. J., vol. 108, no. December, pp. 165-173, 2017.

[21] H. Fayaza, N. A. Rahimb, R. Saidura, K. H. S. H. Niazc, and M. S. Hossaina, "Solar Energy Policy: Malaysia VS Developed Countries," in IEEE Conference on Clean Energy and Technology (CET), 2011, pp. 374-378.

[22] J. S. Overett, "Assessment of the harmonic behaviour of a utility-scale photovoltaic plant," University of Stellenbosch, 2017.

[23] P. A. B. Block et al., "Power quality analyses of a large scale photovoltaic system," in IREC 2014 - 5th International Renewable Energy Congress, 2014.

[24] M. Pourarab, J. Meyer, and R. Stiegler, "Assessment of Harmonic Contribution of a Photovoltaic Installation Based on Field Measurements," in International Conference on Renewable Energies and Power Quality, 2017.

[25] D. Patel, R. K. Varma, R. Seethapathy, and M. Dang, "IMPACT OF WIND TURBINE GENERATORS ON NETWORK RESONANCE," 23rd Can. Conf. Electr. Comput. Eng. (CCECE), no. 1, pp. 1-6, 2010.

[26] A. Honrubia-Escribano, T. García-Sánchez, E. Gómez-Lázaro, E. Muljadi, and A. MolinaGarcía, "Power quality surveys of photovoltaic power plants: Characterisation and analysis of grid-code requirements," IET Renew. Power Gener., vol. 9, no. 5, pp. 466-473, 2015.

[27] M. Ndreko, A. M. Bucurenciu, M. Popov, and M. A. M. M. Van Der Meijden, "On grid code compliance of offshore mtdc grids: Modeling and analysis," 2015 IEEE Eindhoven PowerTech, PowerTech 2015, pp. 1-6, 2015.

[28] P. Haigh, J. McCullagh, and D. M. Joseph, "Ensuring grid code harmonic compliance of wind farms," 10th IET Int. Conf. AC DC Power Transm. (ACDC 2012), pp. 61-61, 2012.

[29] E. Diskin and D. Hynes, "Evidence of the technico-economic impact of energy policy and European Network Codes on distribution system management," in Cigre Dublin Symposium, 2017.

[30] K. L. Koo and Z. Emin, "Harmonic specification for Offshore Wind Farm connections determination, issues and recommendations," in Cigré Dublin Symposium, 2017.

[31] K. Ma, Power Electronics for the Next Generation Wind Turbine System. Aalborg: Springer, 2013.

[32] C. MADTHARAD and J. WARMAN, "Impact of first wind farm in the Kingdom of Thailand on power quality and mitigation solution.," in CIGRE PARIS SESSION, 2014. 
[33] K. Yang, M. H. J. Bollen, E. O. A. Larsson, and M. Wahlberg, "Measurements of harmonic emission versus active power from wind turbines," Electr. Power Syst. Res., vol. 108, pp. 304-314, 2014.

[34] S. Liang, Q. Hu, and W. Lee, "A Survey of Harmonic Emissions of a Commercially Operated Wind Farm," IEEE Trans. Ind. Appl., vol. 48, no. 3, pp. 1115-1123, 2012.

[35] C. F. Jensen, L. H. Kocewiak, and Z. Emin, "Amplification of Harmonic Background Distortion in Wind Power Plants with Long High Voltage Connections (C4-112)," Cigré Sci. Eng. J., no. February, pp. 109-116, 2017.

[36] F. Cavazzana, T. Caldognetto, P. Mattavelli, M. Corradin, and I. Toigo, "Analysis of Current Control Interaction of Multiple Parallel Grid-Connected Inverters," IEEE Trans. Sustain. Energy, vol. 9, no. 4, pp. 1740-1749, 2018.

[37] IEC, "IEC 61727 Photovoltaic (PV) systems - Characteristics of the utility interface," 2002.

[38] IEC, "IEC 61000-3-2 Electromagnetic compatibility (EMC) Part 3-2: Limits - Limits for harmonic current emissions (equipment input current < 16 A per phase), 2014.

[39] IEC, "IEC 61000-3-12: Electromagnetic compatibility (EMC) - Part 3-12: Limits - Limits for harmonic currents produced by equipment connected to public low-voltage systems with input current $>16$ A and $\leq 75$ A per phase," 2011.

[40] IEEE Standards Coordinating Committee 21, IEEE Application Guide for IEEE Std $1547^{T M}$, IEEE Standard for Interconnecting Distributed Resources with Electric Power Systems, no. April. 2009.

[41] CENELEC, "EN 50160 Voltage characteristics of electricity supplied by public distribution networks," 2010.

[42] X. Zong, P. A. Gray, and P. W. Lehn, "New Metric Recommended for IEEE Standard 1547 to Limit Harmonics Injected into Distorted Grids," IEEE Trans. Power Deliv., vol. 31, no. 3, pp. 963-972, 2016.

[43] G. Chicco, J. Schlabbach, and F. Spertino, "Experimental assessment of the waveform distortion in grid-connected photovoltaic installations," Sol. Energy, vol. 83, no. 7, pp. 1026-1039, 2009.

[44] X. Xu et al., "Harmonic emission of PV inverters under different voltage supply conditions and operating powers," in Proceedings of International Conference on Harmonics and Quality of Power, ICHQP, 2016, vol. 2016-Decem.

[45] X. Xu et al., "Aggregate harmonic fingerprint models of PV inverters. part 1: Operation at different powers," Proc. Int. Conf. Harmon. Qual. Power, ICHQP, vol. 2018-May, pp. 1$6,2018$.

[46] S. Müller, J. Meyer, and P. Schegner, "Characterization of small photovoltaic inverters for harmonic modeling," Proc. Int. Conf. Harmon. Qual. Power, ICHQP, pp. 659-663, 2014.

[47] T. H. Ortmeyer, K. R. Chakravarthi, and A. A. Mahmoud, "The Effects of Power System Harmonics On Power System Equipment and Loads," IEEE Trans. Power Appar. Syst., vol. PAS-104, no. 9, pp. 2555-2563, 1985.

[48] IEC, "IEC 61400-21 : 2008 Wind turbines Part 21 : Measurement and assessment of power quality characteristics of grid connected wind turbines," 2008.

[49] IEC, "IEC61000-4-30 Electromagnetic Compatibility (EMC) - Part 4-30: Testing and measurement techniques - Power quality measurement methods," Geneva, Switzerland, 
2015.

[50] Y. Xiao and X. Yang, "Harmonic Summation and Assessment Based on Probability Distribution," Power Deliv. IEEE Trans., vol. 27, no. 2, pp. 1030-1032, 2012.

[51] R. Kniel, P. a. Schnieper, and W. G. Sherman, "Summation of harmonics with random phase angles," Proc. Inst. Electr. Eng., vol. 121, no. 7, p. 708, 1974.

[52] B. Peterson, J. Rens, G. Botha, and U. Minnaar, "Harmonic emission assessment in a dynamic environment using aggregated harmonic phasors," in CIGRE 9th Southern Africa Regional Conference, 2019, no. October, pp. 1-11.

[53] I. Papic et al., "A Benchmark Test System to Evaluate Methods of Harmonic Contribution Determination," IEEE Trans. Power Deliv., vol. 8977, no. c, pp. 1-8, 2018.

[54] F. M. Fernandez and P. S. Chandramohanan Nair, "Estimation of supply side harmonics by using network impedance data," in 2010 Joint International Conference on Power Electronics, Drives and Energy Systems, PEDES 2010 and 2010 Power India, 2010, pp. 16.

[55] O. Gonbeau, J. Javerzac, and D. Boudou, "METHOD TO DETERMINE CONTRIBUTION OF THE CUSTOMER AND THE POWER SYSTEM," in Cired - 17th International Conference on Electricity Distribution, 2003, no. May, pp. 12-15.

[56] F. Safargholi, K. Malekian, and W. Schufft, "On the Dominant Harmonic Source Identification - Part I: Review of Methods," IEEE Trans. Power Deliv., vol. 33, no. 3, pp. 1268-1277, 2018.

[57] F. Safargholi, K. Malekian, and W. Schufft, "On the Dominant Harmonic Source Identification-Part II: Application and Interpretation of Methods," IEEE Trans. Power Deliv., vol. 33, no. 3, pp. 1278-1287, 2018.

[58] G. D'Antona, C. Muscas, and S. Sulis, "State estimation for the localization of harmonic sources in electric distribution systems," in IEEE Transactions on Instrumentation and Measurement, 2009, vol. 58, no. 5, pp. 1462-1470.

[59] L. Cristaldi, a. Ferrero, and S. Salicone, "A distributed system for electric power quality measurement,” IEEE Trans. Instrum. Meas., vol. 51, no. 4, pp. 776-781, 2002.

[60] C. Muscas, L. Peretto, S. Sulis, and R. Tinarelli, "Implementation of multi-point measurement techniques for PQ monitoring," in IEEE Instrumentation and Measurement Technology Conference, 2004, vol. 3, no. 3, pp. 1626-1631.

[61] W. Xu, X. Liu, and Y. Liu, “An Investigation on the Validity of Power Direction Method for Harmonic Source Determination," IEEE Trans. POWER Deliv., vol. 18, no. 1, pp. 214 219, 2003.

[62] B. . Blažič, T. Pfajfar, and I. Papič, "A Modified Harmonic Current Vector Method for Harmonic Contribution Determination," in Power Systems Conference and Exposition, 2004.

[63] IEEE-SA Standards, "IEEE Standard Definitions for the Measurement of Electrical Power Quantities Under Sinusoidal, Nonsinusoidal, Balanced, or Unbalanced Conditions 1459," 2010 .

[64] F. Xu, H. Yang, J. Zhao, Z. Wang, and Y. Liu, "Study on constraints for harmonic source determination using active power direction," IEEE Trans. Power Deliv., vol. 8977, no. c, pp. 1-1, 2018. 
[65] N. Kanao, M. Yamashita, H. Yanagida, M. Mizukami, Y. Hayashi, and J. Matsuki, "Power system harmonic analysis using state-estimation method for Japanese field data," IEEE Trans. Power Deliv., vol. 20, no. 2 I, pp. 970-977, 2005.

[66] C. Rakpenthai, S. Uatrongjit, N. R. Watson, and S. Premrudeepreechacharn, "On Harmonic State Estimation of Power System," IEEE Trans. Power Syst., vol. 28, no. 4, pp. 48294838, 2013.

[67] D. Committee, I. Power, and E. Society, "IEEE Recommended Practice and Requirements for Harmonic Control in Electric Power Systems IEEE Power and Energy Society," vol. 2014, 2014.

[68] J. Rens, J. H. Hattingh, and S. Rens, "On harmonic pollution metering: Field validation of index-based emission assessment," AMPS 2019 - 2019 10th IEEE Int. Work. Appl. Meas. Power Syst. Proc., pp. 8-13, 2019.

[69] A. Ferrero, M. Prioli, and S. Salicone, "Fuzzy metrology-sound approach to the identification of sources injecting periodic disturbances in electric networks," IEEE Trans. Instrum. Meas., vol. 60, no. 9, pp. 3007-3017, 2011.

[70] W. $\mathrm{Xu}$ and Y. Liu, "A Method for Determining Customer and Utility Harmonic Contributions at the Point of Common Coupling," IEEE Trans. POWER Deliv., vol. 15, no. 2, pp. 804-811, 2000.

[71] T. Pfajfar and I. Papič, "Harmonic emission level estimation based on measurements at the point of evaluation," in IEEE Power and Energy Society General Meeting, 2011, pp. 1-5.

[72] CIGRE/CIRED C4.109 WG, "CIGRE/CIRED 468 Review of Disturbance Emission Assessment Techniques,” 2011.

[73] J. Meyer, A. Blanco Castaneda, M. Domagk, and P. Schegner, "Assessment of Prevailing Harmonic Current Emission in Public Low Voltage Networks," IEEE Trans. Power Deliv., vol. 8977, no. c, pp. 1-1, 2016.

[74] IEC, "IEC 61000-4-7 : 2009 Electromagnetic compatibility ( EMC ) Part 4-7 : Testing and measurement techniques - General guide on harmonics and interharmonics measurements and instrumentation, supply systems and equipment connected thereto.," 2009.

[75] M. Calin, A. M. Dumitrescu, M. Asprou, E. Kyriakides, and M. Albu, "Measurement Data Aggregation for Active Distribution Networks," Appl. Meas. Power Syst. (AMPS), IEEE Int. Work., pp. 144-149, 2013.

[76] W. Xu, R. Bahry, H. E. Mazin, and T. Tayjasanant, "A method to determine the harmonic contributions of multiple loads,” 2009 IEEE Power Energy Soc. Gen. Meet. PES '09, pp. $1-6,2009$.

[77] P. S. Wright, A. E. Christensen, P. N. Davis, and T. Lippert, "Multiple-site amplitude and phase measurements of harmonics for analysis of harmonic propagation on bornholm island," IEEE Trans. Instrum. Meas., vol. 66, no. 6, pp. 1176-1183, 2017.

[78] O. Unsar, O. Salor, I. Cadirci, and S. Member, "Identification of Harmonic Current Contributions of Iron and Steel Plants Based on Time-Synchronized Field Measurements — Part I : At PCC," IEEE Trans. Ind. Appl., vol. 50, no. 6, pp. 4336-4347, 2014.

[79] A. Carta, N. Locci, and C. Muscas, "GPS-Based System for the Measurement of Synchronized Harmonic Phasors," IEEE Trans. Instrum. Meas., vol. 58, no. 3, 2009.

[80] A. M. Blanco, R. Stiegler, J. Meyer, and M. Schwenke, "Implementation of harmonic phase angle measurement for power quality instruments," IEEE Int. Work. Appl. Meas. Power 
Syst., pp. 5-10, 2016.

[81] Joint Committee for Guides in Metrology, "Evaluation of measurement data - Guide to the expression of uncertainty in measurement," 2008.

[82] A. Ferrero, D. Petri, P. Carbone, and M. Catelani, Modern Measurements: Fundamentals and Applications, First Edit. John Wiley \& Sons Ltd, 2015.

[83] L. Kadar, P. Hacksel, and J. Wikston, "The effect of current and voltage transformers accuracy on harmonic measurements in electric arc furnaces," IEEE Trans. Ind. Appl., vol. 33, no. 3, pp. 780-783, 1997.

[84] M. Klatt, J. Meyer, M. Elst, and P. Schegner, "Frequency Responses ofMV voltage transformers in the range of $50 \mathrm{~Hz}$ to $10 \mathrm{kHz}, " 14$ th Int. Conf. Harmon. Qual. Power, pp. 16, 2010.

[85] J. Meyer, R. Stiegler, M. Elst, E. Sperling, and M. Klatt, “Accuracy Of Harmonic Voltage Measurements in the Frequency Range Up To $5 \mathrm{kHz}$ Using Conventional Instrument Transformers," in 21st International Conference on Electricity Distribution - CIRED, 2011, vol. 21, pp. 6-9.

[86] S. Zhao, H. Li, P. Crossley, and F. Ghassemi, "Test and Analysis of Harmonic Responses of High Voltage Instrument Voltage Transformers," in 12th IET International Conference on Developments in Power System Protection, 2014, pp. 1-6.

[87] IEC, IEC/TR 61869-103:2012 Technical report Instrument transformers - The use of instrument transformers for power quality measurement. 2012.

[88] NRS, "SANS 474 : 2009 SOUTH AFRICAN NATIONAL STANDARD Code of practice for electricity metering." 2009.

[89] F. R. Quintela, R. C. Redondo, N. R. Melchor, and M. Redondo, "A General Approach to Kirchhoff' s Laws," IEEE Trans. Educ., vol. 52, no. 2, pp. 273-278, 2009.

[90] F. Meng, X. Xu, and L. Gao, "A Harmonic Reduction Method in Multi-Pulse Rectifier Using Passive Devices," IEEE Trans. Ind. INFORMATICS, vol. 32, no. 5, pp. 77-86, 2017.

[91] S. Li et al., "PMU-based Harmonic Phasor Calculation and Harmonic Source Identification," 2nd IEEE Conf. Energy Internet Energy Syst. Integr. EI2 2018 - Proc., pp. $1-6,2018$.

[92] K. Behrendt, K. Fodero, and S. E. Laboratories, "The Perfect Time: An Examination of Time Synchronization Techniques," pp. 1-18, 2003.

[93] B. Peterson, J. Rens, U. Minnaar, G. Botha, and J. Desmet, "A South African Review of Harmonic Emission Level Assessment As Per IEC61000-3-6," in CIGRE, 2015, pp. 1-9.

[94] C. Chen, X. Liu, D. Koval, W. Xu, and T. Tayjasanant, "Critical Impedance Method - A New detecting Harmonic Sources Method in Distribution Systems," IEEE Trans. Power Deliv., vol. 19, no. 1, pp. 288-297, 2004.

[95] U. Minnaar, B. Peterson, H. Mostert, J. Rens, and G. Botha, "Power Quality Grid Code Compliance for Renewable Power Plants in South Africa," IET Gener. Transm. Distrib., Nov. 2018.

[96] Z. Marais, J. Rens, B. Peterson, and G. Botha, "Measurement Uncertainty of Current Harmonics During the Assessment of Compliance to Grid Code Requirements," in 2018 IEEE 9th International Workshop on Applied Measurements for Power Systems (AMPS), 2018, pp. 1-6. 
[97] IEC, “IEC 61869-2 Instrument transformers - Part 2: Additional requirements for current transformers," 2012.

[98] B. Peterson, J. Rens, G. Botha, and J. Desmet, "A discriminative approach to harmonic emission assessment," in IEEE International Workshop on Applied Measurements for Power Systems (AMPS), 2015.

[99] S. Müller, J. Meyer, F. Möller, M. Naumann, and M. Radauer, "Impact of a High Penetration of Electric Vehicles and Photovoltaic Inverters on Power Quality in an Urban Residential Grid Part II - Harmonic Distortion Key words," in International Conference on Renewable Energies and Power Quality - ICREPQ 2016, 2016, no. 14.

[100] S. Müller, F. Möller, J. Meyer, A. J. Collin, and S. Z. Djokic, "Characterisation of harmonic interactions between electric vehicle battery chargers and PV inverters," Proc. Int. Conf. Harmon. Qual. Power, ICHQP, pp. 645-649, 2014.

[101] D. Committee, IEEE Std 1531 TM-2003 IEEE: IEEE Standards IEEE Standards 1531 TM IEEE Guide for Application and Specification of Harmonic Filters, vol. 2009, no. June. 2003.

[102] D. Serfontein, J. Rens, G. Botha, and J. Desmet, "Continuous Event-Based Harmonic Impedance Assessment Using Online Measurements," IEEE Trans. Instrum. Meas., vol. 65, no. 10, pp. 2214-2220, 2016.

[103] B. Peterson, J. Rens, J. Meyer, G. Botha, and J. Desmet, “On The Assessment of Harmonic Emission in Distribution Networks: Opportunity for the Prevailing Harmonic Phase Angle," in IEEE International Workshop on Applied Measurements for Power Systems (AMPS), 2016, pp. 3-8.

[104] A. Varatharajan, S. Schoettke, J. Meyer, and A. Abart, "Harmonic Emission of Large PV Installations Case Study of a 1 MW Solar Campus," Int. Conf. Renew. Energy Power Qual. $J .$, no. April 2014, 2014.

[105] J. Meyer, M. Domagk, T. Darda, and G. Eberl, "Influence of aggregation intervals on power quality assessment according to EN 50160," in 22nd International Conference and Exhibition on Electricity Distribution (CIRED 2013), 2013, vol. 5, no. 1174, pp. 11741174.

[106] S. Elphick, V. Gosbell, and S. Perera, "The effect of data aggregation interval on voltage results," 2007 Australas. Univ. Power Eng. Conf., 2007.

[107] L. F. Beites, J. G. Mayordomo, A. Hernandez, and R. Asensi, "Harmonics, interharmonics and unbalances of arc furnaces: A new frequency domain approach," IEEE Trans. POWER Deliv., vol. 14, no. 4, pp. 661-668, 2001.

[108] IEC, "Electromagnetic compatibility - Part 1-8: General - Phase angles of harmonic current emissions and voltages in the public supply networks - Future expectations," 2019.

[109] N. Cho, "Allocation Of Individual Harmonic Emission Limits In Accordance With The Principles Of IEC / TR 61000-3-6," Georgia Institute of Technology, 2013.

[110] B. Peterson, J. Rens, G. Botha, and J. Desmet, "A Discriminative Approach to Harmonic Emission Assessment," in IEEE International Workshop on Applied Measurements for Power Systems (AMPS), 2015, no. June.

[111] B. Peterson, J. Rens, J. Meyer, G. Botha, and J. Desmet, "On the assessment of harmonic emission in distribution networks: Opportunity for the prevailing harmonic phase angle," in 2016 IEEE International Workshop on Applied Measurements for Power Systems, AMPS 
2016 - Proceedings, 2016.

[112] B. Peterson, J. Rens, G. Botha, J. Desmet, and S. Rens, "Tracking harmonic filter performance using aggregated harmonic phasors," Africa Res. J., no. October, 2019.

[113] B. Peterson, J. Rens, and J. Desmet, "Harmonic Emission Assessment on a Distribution Network : the Opportunity for the Prevailing Angle in Harmonic Phasors," in CIRED - 24th International Conference on Electricity Distribution, 2017, no. June.

[114] B. Peterson, J. Rens, and J. Desmet, "Harmonic emission assessment on a distribution network: the opportunity for the prevailing angle in harmonic phasors," CIRED - Open Access Proc. J. - IET, vol. 2017, no. 1, p. 4, 2017.

[115] B. Peterson, J. Rens, J. Meyer, G. Botha, and J. Desmet, "Evaluation of Harmonic Distortion from Multiple Renewable Sources at a Distribution Substation," in IEEE International Workshop on Applied Measurements for Power Systems (AMPS) Proceedings, 2017, pp. 37-42.

[116] B. Peterson, A. M. Blanco, J. Rens, J. Meyer, G. Botha, and J. Desmet, "Impact of Aggregation Interval on Harmonic Phase Angle Measurements," in 2018 IEEE 9th International Workshop on Applied Measurements for Power Systems (AMPS), 2018, pp. $1-6$. 


\section{APPENDIX A: VERIFICATION OF ADDITIONAL HARMONIC ORDERS}

The following section shows the results of the $5^{\text {th }}, 7^{\text {th }}, 11^{\text {th }}$ and $13^{\text {th }}$ harmonic orders for the various methods discussed in Chapter 3:. Note that the only variable that was changed was the frequency to obtain the results for the different harmonic order, as no capacitance was modelled the impedances are linear. None of the input values were altered to ensure consistency in the various harmonic emission assessment methods applied.

\section{Harmonic emission}

Table A1 to Table A3 shows the voltage harmonic emission results for the $7^{\text {th }}, 11^{\text {th }}$ and $13^{\text {th }}$ harmonic orders respectively.

Table A1. Voltage harmonic emission for $7^{\text {th }}$ harmonic.

\begin{tabular}{|c|c|c|c|}
\hline \multicolumn{2}{|c|}{${7^{\text {th }}}^{2}$ calculated } & \multicolumn{2}{c|}{${7^{\text {th }}}^{\text {E }}$ calculated } \\
\hline Mag [V] & Angle [ ${ }^{\circ}$ ] & Mag [V] & Angle [ $\left.{ }^{\circ}\right]$ \\
\hline 202.02 & 32.19 & 7.94 & -73.63 \\
\hline 214.93 & 31.90 & 16.43 & -124.31 \\
\hline 227.84 & 31.64 & 28.50 & -136.76 \\
\hline 225.32 & 35.83 & 33.28 & -106.53 \\
\hline 231.36 & 36.42 & 39.54 & -109.13 \\
\hline 237.41 & 36.98 & 45.86 & -111.02 \\
\hline 199.35 & 33.87 & 13.51 & -55.32 \\
\hline 203.27 & 35.34 & 19.07 & -67.19 \\
\hline 207.32 & 36.75 & 25.07 & -73.56 \\
\hline
\end{tabular}

Table A2. Voltage harmonic emission for $11^{\text {th }}$ harmonic.

\begin{tabular}{|c|c|c|c|}
\hline \multicolumn{2}{|c|}{$\mathbf{1 1}^{\text {th }} \boldsymbol{V}_{h}$ calculated } & \multicolumn{2}{c|}{$\mathbf{1 1}^{\text {th }} \boldsymbol{E}_{h c}$ calculated } \\
\hline Mag [V] & Angle [ ${ }^{\circ}$ ] & Mag [V] & Angle [ $\left.{ }^{\circ}\right]$ \\
\hline 203.12 & 31.72 & 6.81 & -86.35 \\
\hline 217.61 & 32.13 & 19.24 & -125.19 \\
\hline 232.12 & 32.48 & 33.45 & -132.54 \\
\hline 226.28 & 36.96 & 36.85 & -101.90 \\
\hline 232.57 & 37.88 & 44.04 & -103.60 \\
\hline 238.92 & 38.75 & 51.26 & -104.83 \\
\hline 198.92 & 33.42 & 11.95 & -53.10 \\
\hline 202.18 & 35.28 & 18.66 & -64.06 \\
\hline 205.65 & 37.08 & 25.68 & -69.14 \\
\hline
\end{tabular}

Table A3. Voltage harmonic emission for $13^{\text {th }}$ harmonic.

\begin{tabular}{|c|c|c|c|}
\hline \multicolumn{2}{|c|}{$\mathbf{1 3}^{\text {th }} \boldsymbol{V}_{h}$ calculated } & \multicolumn{2}{c|}{$\mathbf{1 3}^{\text {th }} \boldsymbol{E}_{h c}$ calculated } \\
\hline Mag [V] & Angle [ $^{\circ}$ ] & Mag [V] & Angle [ $\left.{ }^{\circ}\right]$ \\
\hline 203.57 & 31.68 & 6.92 & -90.23 \\
\hline 218.89 & 32.43 & 20.86 & -123.64 \\
\hline 234.23 & 33.07 & 36.14 & -129.69 \\
\hline 226.75 & 37.72 & 39.21 & -99.04 \\
\hline
\end{tabular}




\begin{tabular}{|l|l|l|l|}
233.21 & 38.80 & 46.91 & -100.49 \\
\hline 239.74 & 39.82 & 54.64 & -101.53 \\
\hline 198.61 & 33.39 & 11.87 & -51.58 \\
\hline 201.55 & 35.45 & 19.16 & -61.92 \\
\hline 204.75 & 37.45 & 26.73 & -66.49 \\
\hline
\end{tabular}

\section{Harmonic complex power}

Table A4 present the harmonic complex values at the PoC for the $5^{\text {th }}, 7^{\text {th }} 11^{\text {th }}$ and $13^{\text {th }}$ harmonic orders for the scenario when the harmonic source is in the load only.

Table A4. Harmonic complex power (source in load only).

\begin{tabular}{|c|c|c|c|c|c|c|c|}
\hline \multicolumn{8}{|c|}{ Harmonic complex power } \\
\hline \multicolumn{2}{|c|}{$5^{\text {th }}$ harmonic } & \multicolumn{2}{|c|}{$7^{\text {th }}$ harmonic } & \multicolumn{2}{|c|}{$11^{\text {th }}$ harmonic } & \multicolumn{2}{|c|}{$13^{\text {th }}$ harmonic } \\
\hline Mag [VA] & angle $\left[{ }^{\circ}\right]$ & Mag [VA] & angle $\left[{ }^{\circ}\right]$ & Mag [VA] & angle $\left[{ }^{\circ}\right]$ & Mag [VA] & angle $\left[^{\circ}\right.$ \\
\hline 76.54 & -82.51 & 70.30 & -101.31 & 70.45 & -119.64 & 71.59 & -124.34 \\
\hline 143.10 & -114.86 & 149.41 & -125.46 & 158.93 & -133.80 & 162.45 & -135.63 \\
\hline 233.71 & -126.51 & 246.10 & -133.17 & 261.56 & -137.88 & 267.26 & -138.68 \\
\hline 279.20 & -91.82 & 276.35 & -97.73 & 275.32 & -101.98 & 275.59 & -102.61 \\
\hline 330.42 & -93.98 & 328.91 & -99.06 & 329.18 & -102.33 & 329.96 & -102.62 \\
\hline 384.40 & -95.56 & 384.10 & -99.96 & 385.69 & -102.43 & 387.06 & -102.44 \\
\hline 134.95 & -49.16 & 117.10 & -56.87 & 101.40 & -65.04 & 97.27 & -67.38 \\
\hline 173.64 & -54.99 & 157.02 & -61.83 & 141.97 & -68.31 & 137.84 & -69.96 \\
\hline 215.68 & -58.41 & 199.72 & -64.35 & 184.67 & -69.46 & 180.33 & -70.56 \\
\hline
\end{tabular}

Table A5 present the harmonic complex values at the PoC for the $5^{\text {th }}, 7^{\text {th }} 11^{\text {th }}$ and $13^{\text {th }}$ harmonic orders for the scenario when the harmonic source is in the network only

Table A5. Harmonic complex power (source in network only).

\begin{tabular}{|c|c|c|c|c|c|c|c|}
\hline \multicolumn{8}{|c|}{ Harmonic complex power } \\
\hline \multicolumn{2}{|c|}{$5^{\text {th }}$ harmonic } & \multicolumn{2}{|c|}{$7^{\text {th }}$ harmonic } & \multicolumn{2}{|c|}{$11^{\text {th }}$ harmonic } & \multicolumn{2}{|c|}{$13^{\text {th }}$ harmonic } \\
\hline Mag [VA] & angle $\left[{ }^{\circ}\right]$ & Mag [VA] & angle $\left[{ }^{\circ}\right]$ & Mag [VA] & angle $\left[{ }^{\circ}\right]$ & Mag [VA] & angle $\left[^{\circ}\right.$ \\
\hline 3.04 & -80.02 & 2.18 & -82.86 & 1.39 & -85.46 & 1.18 & -86.16 \\
\hline 12.16 & -80.02 & 8.73 & -82.85 & 5.58 & -85.45 & 4.72 & -86.15 \\
\hline 27.35 & -80.02 & 19.65 & -82.85 & 12.55 & -85.45 & 10.63 & -86.15 \\
\hline 27.35 & -20.02 & 19.65 & -22.85 & 12.55 & -25.44 & 10.63 & -26.15 \\
\hline 37.23 & -20.02 & 26.75 & -22.85 & 17.08 & -25.44 & 14.47 & -26.14 \\
\hline 48.63 & -20.02 & 34.94 & -22.85 & 22.31 & -25.44 & 18.89 & -26.14 \\
\hline 3.04 & 39.98 & 2.18 & 37.15 & 1.39 & 34.55 & 1.18 & 33.85 \\
\hline 6.84 & 39.98 & 4.91 & 37.15 & 3.14 & 34.55 & 2.66 & 33.85 \\
\hline 12.16 & 39.98 & 8.73 & 37.15 & 5.58 & 34.56 & 4.72 & 33.86 \\
\hline
\end{tabular}

Table A6 present the harmonic complex values at the PoC for the $5^{\text {th }}, 7^{\text {th }} 11^{\text {th }}$ and $13^{\text {th }}$ harmonic orders for the scenario when the harmonic source is in both the network and the load.

Table A6. Harmonic complex power (source in network and load) 


\begin{tabular}{|c|c|c|c|c|c|c|c|}
\hline \multicolumn{2}{|c|}{$5^{\text {th }}$ harmonic } & \multicolumn{2}{c|}{$7^{\text {th }}$ harmonic } & \multicolumn{2}{c|}{$\mathbf{1 1}^{\text {th }}$ harmonic } & \multicolumn{2}{c|}{ 13 $^{\text {th }}$ harmonic $^{\prime}$} \\
\hline Mag [VA] & angle [ ${ }^{\circ}$ ] & Mag [VA] & angle [ ${ }^{\circ}$ ] & Mag [VA] & angle [ ${ }^{\circ}$ & Mag [VA] & angle [ ${ }^{\circ}$ \\
\hline 4.52 & -135.62 & 4.74 & -139.11 & 5.02 & -140.66 & 5.14 & -140.45 \\
\hline 69.80 & -151.82 & 69.09 & -157.73 & 68.83 & -161.98 & 68.90 & -162.61 \\
\hline 290.72 & -144.22 & 280.74 & -152.54 & 274.62 & -159.64 & 273.64 & -161.23 \\
\hline 197.50 & -91.51 & 195.32 & -97.53 & 194.47 & -101.91 & 194.63 & -102.59 \\
\hline 418.64 & -84.22 & 404.26 & -92.54 & 395.45 & -99.64 & 394.04 & -101.23 \\
\hline 493.10 & -49.83 & 509.85 & -62.04 & 541.15 & -71.92 & 553.16 & -74.17 \\
\hline 6.01 & -8.79 & 6.32 & -14.67 & 6.72 & -18.63 & 6.86 & -19.20 \\
\hline 106.92 & -94.30 & 93.57 & -98.94 & 80.77 & -103.40 & 77.09 & -104.57 \\
\hline 161.96 & -95.26 & 144.78 & -99.51 & 127.75 & -103.27 & 122.65 & -104.15 \\
\hline
\end{tabular}

\section{CIGRE C4.109 scatterplot}

Table A7 shows the CIGRE scatterplot results $\left(\boldsymbol{V}_{h}\right.$ and $\left.\boldsymbol{I}_{h}\right)$ at the PoC for the $5^{\text {th }}, 7^{\text {th }} 11^{\text {th }}$ and $13^{\text {th }}$ harmonic orders for the scenario when the harmonic source is in the load only.

Table A7. CIGRE C4.109 scatterplot (source in load only)

\begin{tabular}{|c|c|c|c|c|c|c|c|}
\hline \multicolumn{2}{|c|}{$\mathbf{5}^{\text {th }}$ harmonic } & \multicolumn{2}{c|}{${7^{\text {th }}}^{2}$ harmonic } & \multicolumn{2}{c|}{$\mathbf{1 1}^{\text {th }}$ harmonic } & \multicolumn{2}{c|}{$\mathbf{1 3}^{\text {th }}$ harmonic } \\
\hline $\boldsymbol{I}_{h} \mathrm{Mag}[\mathrm{A}]$ & $\boldsymbol{V}_{h}$ Mag [V] & $\boldsymbol{I}_{h} \mathrm{Mag}[\mathrm{A}]$ & $\boldsymbol{V}_{h} \mathrm{Mag}[\mathrm{V}]$ & $\boldsymbol{I}_{h} \mathrm{Mag}[\mathrm{A}]$ & $\boldsymbol{V}_{h} \mathrm{Mag}[\mathrm{V}]$ & $\boldsymbol{I}_{h} \mathrm{Mag}[\mathrm{A}]$ & $\boldsymbol{V}_{h} \mathrm{Mag}[\mathrm{V}$ \\
\hline 0.19 & 7.13 & 0.19 & 7.45 & 0.20 & 8.26 & 0.20 & 8.74 \\
\hline 0.58 & 19.46 & 0.59 & 20.40 & 0.59 & 22.83 & 0.59 & 24.30 \\
\hline 0.97 & 31.80 & 0.98 & 33.35 & 0.98 & 37.41 & 0.98 & 39.85 \\
\hline 0.98 & 31.77 & 0.98 & 33.26 & 0.98 & 37.23 & 0.98 & 39.64 \\
\hline 1.17 & 37.94 & 1.17 & 39.74 & 1.18 & 44.51 & 1.18 & 47.41 \\
\hline 1.37 & 44.11 & 1.37 & 46.21 & 1.37 & 51.80 & 1.37 & 55.19 \\
\hline 0.20 & 6.86 & 0.20 & 7.08 & 0.20 & 7.73 & 0.20 & 8.15 \\
\hline 0.39 & 13.01 & 0.39 & 13.53 & 0.39 & 14.99 & 0.39 & 15.90 \\
\hline 0.59 & 19.17 & 0.59 & 20.00 & 0.59 & 22.27 & 0.59 & 23.67 \\
\hline
\end{tabular}

Table A8 shows the CIGRE scatterplot results $\left(\boldsymbol{V}_{h}\right.$ and $\left.\boldsymbol{I}_{h}\right)$ at the PoC for the $5^{\text {th }}, 7^{\text {th }} 11^{\text {th }}$ and $13^{\text {th }}$ harmonic orders for the scenario when the harmonic source is in the network only.

Table A8. CIGRE C4.109 scatterplot (source in the network only)

\begin{tabular}{|c|c|c|c|c|c|c|c|}
\hline \multicolumn{2}{|c|}{$5^{\text {th }}$ harmonic } & \multicolumn{2}{|c|}{$7^{\text {th }}$ harmonic } & \multicolumn{2}{|c|}{$11^{\text {th }}$ harmonic } & \multicolumn{2}{|c|}{$13^{\text {th }}$ harmonic } \\
\hline 0.04 & 19.51 & 0.03 & 19.56 & 0.02 & 19.59 & 0.02 & 19.59 \\
\hline 0.12 & 58.53 & 0.08 & 58.67 & 0.05 & 58.76 & 0.05 & 58.78 \\
\hline 0.19 & 97.54 & 0.14 & 97.78 & 0.09 & 97.94 & 0.08 & 97.97 \\
\hline 0.27 & 136.56 & 0.20 & 136.90 & 0.12 & 137.11 & 0.11 & 137.15 \\
\hline 0.04 & 19.51 & 0.03 & 19.56 & 0.02 & 19.59 & 0.02 & 19.59 \\
\hline 0.08 & 39.02 & 0.06 & 39.11 & 0.04 & 39.17 & 0.03 & 39.19 \\
\hline 0.12 & 58.53 & 0.08 & 58.67 & 0.05 & 58.76 & 0.05 & 58.78 \\
\hline
\end{tabular}


Table A10 shows the CIGRE scatterplot results $\left(\boldsymbol{V}_{h}\right.$ and $\left.\boldsymbol{I}_{h}\right)$ at the PoC for the $5^{\text {th }}, 7^{\text {th }} 11^{\text {th }}$ and $13^{\text {th }}$ harmonic orders for the scenario when the harmonic source is in the network and the load.

Table A10. CIGRE C4.109 scatterplot (source in the network and the load)

\begin{tabular}{|c|c|c|c|c|c|c|r|}
\hline \multicolumn{2}{|c|}{$\mathbf{5}^{\text {th }}$ harmonic } & \multicolumn{2}{c|}{${7^{\text {th }} \text { harmonic }}^{2} \mathbf{1 1}^{\text {th }}$ harmonic } & \multicolumn{2}{c|}{$\mathbf{1 3}^{\text {th }}$ harmonic } \\
\hline $\boldsymbol{I}_{h} \mathrm{Mag}[\mathrm{A}]$ & $\boldsymbol{V}_{h} \mathrm{Mag}[\mathrm{V}]$ & $\boldsymbol{I}_{h} \mathrm{Mag}[\mathrm{A}]$ & $\boldsymbol{V}_{h} \mathrm{Mag}[\mathrm{V}]$ & $\boldsymbol{I}_{h} \mathrm{Mag}[\mathrm{A}]$ & $\boldsymbol{V}_{h} \mathrm{Mag}[\mathrm{V}]$ & $\boldsymbol{I}_{h} \mathrm{Mag}[\mathrm{A}]$ & $\boldsymbol{V}_{h} \mathrm{M}$ \\
\hline 0.18 & 25.58 & 0.18 & 26.01 & 0.19 & 26.84 & 0.19 & 27 \\
\hline 0.61 & 115.25 & 0.60 & 115.68 & 0.59 & 116.28 & 0.59 & 116 \\
\hline 1.06 & 273.29 & 1.02 & 274.14 & 1.00 & 275.07 & 0.99 & 275 \\
\hline 1.01 & 195.33 & 1.00 & 196.05 & 0.99 & 197.05 & 0.99 & 197 \\
\hline 1.28 & 327.95 & 1.23 & 328.97 & 1.20 & 330.08 & 1.19 & 330 \\
\hline 1.14 & 432.57 & 1.17 & 436.31 & 1.22 & 442.48 & 1.24 & 445 \\
\hline 0.17 & 35.33 & 0.18 & 35.79 & 0.18 & 36.63 & 0.19 & 37 \\
\hline 0.67 & 159.00 & 0.59 & 158.11 & 0.52 & 155.78 & 0.50 & 154 \\
\hline 0.90 & 180.20 & 0.81 & 178.75 & 0.73 & 175.26 & 0.71 & 173 \\
\hline
\end{tabular}

\section{Harmonic vector method}

Table A11 shows the harmonic vector method results for the $5^{\text {th }}$ and $7^{\text {th }}$ harmonic currents.

Table A11. Harmonic Vector method for the $5^{\text {th }}$ and $7^{\text {th }}$ harmonics

\begin{tabular}{|c|c|c|c|c|c|c|c|c|c|}
\hline \multicolumn{6}{|c|}{$5^{\text {th }}$ harmonic } & \multicolumn{4}{|c|}{$7^{\text {th }}$ harmonic } \\
\hline \multicolumn{2}{|c|}{$\boldsymbol{I}_{h}$} & \multicolumn{2}{|c|}{$\mathbf{I}_{\mathrm{hc}}$} & \multicolumn{2}{|c|}{$\boldsymbol{I}_{h 0}$} & \multicolumn{2}{|c|}{$\boldsymbol{I}_{h}$} & \multicolumn{2}{|c|}{$\mathbf{I}_{\mathrm{hc}}$} \\
\hline $\operatorname{Mag}[\mathbf{A}]$ & angle $\left[{ }^{\circ}\right]$ & Mag [A] & angle $\left[{ }^{\circ}\right]$ & Mag [A] & angle $\left[{ }^{\circ}\right]$ & Mag [A] & angle $\left[{ }^{\circ}\right]$ & Mag [A] & al \\
\hline 0.33 & -83.29 & 0.18 & -0.96 & 6.23 & 9.30 & 0.33 & -83.29 & 0.15 & \\
\hline 0.49 & -135.48 & 0.58 & 1.68 & 5.93 & 10.56 & 0.49 & -135.48 & 0.52 & \\
\hline 0.83 & -153.59 & 0.98 & 2.17 & 5.65 & 11.96 & 0.83 & -153.59 & 0.92 & \\
\hline 1.03 & -124.51 & 0.98 & 32.73 & 5.58 & 7.78 & 1.03 & -124.51 & 0.97 & \\
\hline 1.21 & -128.00 & 1.17 & 32.76 & 5.42 & 7.57 & 1.21 & -128.00 & 1.17 & \\
\hline 1.40 & -130.57 & 1.37 & 32.78 & 5.26 & 7.33 & 1.40 & -130.57 & 1.36 & \\
\hline 0.51 & -73.39 & 0.18 & 65.34 & 6.24 & 7.84 & 0.51 & -73.39 & 0.25 & \\
\hline 0.66 & -85.04 & 0.38 & 64.05 & 6.12 & 6.93 & 0.66 & -85.04 & 0.44 & \\
\hline 0.84 & -92.08 & 0.58 & 63.65 & 5.99 & 5.98 & 0.84 & -92.08 & 0.63 & \\
\hline
\end{tabular}

Table A12 shows the harmonic vector method results for the $11^{\text {th }}$ and $13^{\text {th }}$ harmonic currents.

Table A12. Harmonic Vector method for the $11^{\text {th }}$ and $13^{\text {th }}$ harmonics

\begin{tabular}{|c|c|c|c|c|c|c|c|c|c|}
\hline \multicolumn{6}{|c|}{$11^{\text {th }}$ harmonic } & \multicolumn{4}{|c|}{$13^{\text {th }}$ harmo } \\
\hline \multicolumn{2}{|c|}{$\boldsymbol{I}_{h}$} & \multicolumn{2}{|c|}{$\mathbf{I}_{\mathrm{hc}}$} & \multicolumn{2}{|c|}{$\boldsymbol{I}_{h 0}$} & \multicolumn{2}{|c|}{$\boldsymbol{I}_{h}$} & \multicolumn{2}{|c|}{$\mathbf{I}_{\mathrm{hc}}$} \\
\hline Mag [A] & angle $\left[{ }^{\circ}\right]$ & Mag [A] & angle $\left[{ }^{\circ}\right]$ & $\operatorname{Mag}[\mathbf{A}]$ & angle $\left[{ }^{\circ}\right]$ & Mag [A] & angle $\left[{ }^{\circ}\right]$ & $\operatorname{Mag}[\mathbf{A}]$ & al \\
\hline 0.33 & -83.29 & 0.18 & 71.37 & 5.39 & -8.92 & 0.33 & -83.29 & 0.20 & \\
\hline 0.49 & -135.48 & 0.49 & 23.14 & 5.09 & -8.98 & 0.49 & -135.48 & 0.49 & \\
\hline 0.83 & -153.59 & 0.87 & 14.22 & 4.79 & -9.05 & 0.83 & -153.59 & 0.86 & \\
\hline 1.03 & -124.51 & 0.98 & 45.51 & 4.91 & -13.41 & 1.03 & -124.51 & 0.98 & \\
\hline 1.21 & -128.00 & 1.17 & 43.44 & 4.79 & -14.46 & 1.21 & -128.00 & 1.18 & \\
\hline 1.40 & -130.57 & 1.37 & 41.95 & 4.67 & -15.56 & 1.40 & -130.57 & 1.37 & \\
\hline
\end{tabular}




\begin{tabular}{|l|l|l|l|l|l|l|l|l|l|}
0.51 & -73.39 & 0.34 & 98.39 & 5.47 & -10.27 & 0.51 & -73.39 & 0.37 & 0.54 \\
\hline 0.66 & -85.04 & 0.51 & 85.59 & 5.40 & -11.68 & 0.66 & -85.04 & -92.08 & 0.72 \\
\hline 0.84 & -92.08 & 0.70 & 79.40 & 5.34 & -13.13 & 0.84 & & \\
\hline
\end{tabular}

\section{Range of secondary cancellation}

Input values used in section 3.7.

Table A13 shows the input values used for the range of secondary cancellation when the harmonic source exists in the load.

Table A13. Input values used for the range of secondary cancellation when harmonic source is in the network only.

\begin{tabular}{|c|c|}
\hline \multicolumn{2}{|c|}{$\boldsymbol{I}_{h c}$} \\
\hline Mag $[\mathbf{A}]$ & Angle $\left[^{\circ}\right]$ \\
\hline 0.2 & 0 \\
\hline 0.4 & 0 \\
\hline 0.6 & 0 \\
\hline 0.8 & 0 \\
\hline 1 & 0 \\
\hline 1.2 & 0 \\
\hline 1.4 & 0 \\
\hline 1.6 & 0 \\
\hline 1.8 & 0 \\
\hline 2 & 0 \\
\hline 0.2 & 30 \\
\hline 0.4 & 30 \\
\hline 0.6 & 30 \\
\hline 0.8 & 30 \\
\hline 1 & 30 \\
\hline 1.2 & 30 \\
\hline 1.4 & 30 \\
\hline 1.6 & 30 \\
\hline 1.8 & 30 \\
\hline 2 & 30 \\
\hline 0.2 & 60 \\
\hline 0.4 & 60 \\
\hline 0.6 & 60 \\
\hline 0.8 & 60 \\
\hline 1 & 60 \\
\hline 1.2 & 60 \\
\hline 1.4 & 60 \\
\hline 1.6 & 60 \\
\hline 1.8 & 60 \\
\hline 2 & 60 \\
\hline 0.2 & 90 \\
\hline
\end{tabular}




\begin{tabular}{|c|c|}
\hline \multicolumn{2}{|c|}{$\boldsymbol{I}_{h c}$} \\
\hline Mag [A] & Angle [ [ $\left.^{\circ}\right]$ \\
\hline 0.4 & 90 \\
\hline 0.6 & 90 \\
\hline 0.8 & 90 \\
\hline 1 & 90 \\
\hline 1.2 & 90 \\
\hline 1.4 & 90 \\
\hline 1.6 & 90 \\
\hline 1.8 & 90 \\
\hline 2 & 90 \\
\hline
\end{tabular}

Table A14 shows the input values used for the range of secondary cancellation when the harmonic source exists in the upstream network.

Table A14. Input values used for the range of secondary cancellation when harmonic source is in the network only.

\begin{tabular}{|c|c|}
\hline \multicolumn{2}{|c|}{$\boldsymbol{V}_{h 0}$} \\
\hline Mag [V] & Angle $\left[^{\circ}\right]$ \\
\hline 20 & 0 \\
\hline 40 & 0 \\
\hline 60 & 0 \\
\hline 80 & 0 \\
\hline 100 & 0 \\
\hline 120 & 0 \\
\hline 140 & 0 \\
\hline 160 & 0 \\
\hline 180 & 0 \\
\hline 200 & 0 \\
\hline 20 & 30 \\
\hline 40 & 30 \\
\hline 60 & 30 \\
\hline 80 & 30 \\
\hline 100 & 30 \\
\hline 120 & 30 \\
\hline 140 & 30 \\
\hline 160 & 30 \\
\hline 180 & 30 \\
\hline 200 & 30 \\
\hline 20 & 60 \\
\hline 40 & 60 \\
\hline 60 & 60 \\
\hline 80 & 60 \\
\hline 100 & 60 \\
\hline
\end{tabular}




\begin{tabular}{|c|c|}
\hline \multicolumn{2}{|c|}{$\boldsymbol{V}_{h 0}$} \\
\hline Mag [V] & Angle $\left.^{{ }^{\circ}}{ }^{\circ}\right]$ \\
\hline 120 & 60 \\
\hline 140 & 60 \\
\hline 160 & 60 \\
\hline 180 & 60 \\
\hline 200 & 60 \\
\hline 20 & 90 \\
\hline 40 & 90 \\
\hline 60 & 90 \\
\hline 80 & 90 \\
\hline 100 & 90 \\
\hline 120 & 90 \\
\hline 140 & 90 \\
\hline 160 & 90 \\
\hline 180 & 90 \\
\hline 200 & 90 \\
\hline
\end{tabular}

Table A15 shows the input values used for the range of secondary cancellation when the harmonic source exists in the upstream network and with a reduced angular range.

Table A15. Input values used for the range of secondary cancellation when harmonic source is in the network only with reduced angular range.

\begin{tabular}{|c|c|}
\hline \multicolumn{2}{|c|}{$\boldsymbol{V}_{h 0}$} \\
\hline Mag [V] & Angle $\left[^{\circ}\right]$ \\
\hline 41 & 30 \\
\hline 40 & 34 \\
\hline 44 & 35 \\
\hline 43 & 36 \\
\hline 34 & 32 \\
\hline 36 & 34 \\
\hline 37 & 35 \\
\hline 38 & 34 \\
\hline 39 & 40 \\
\hline 33 & 32 \\
\hline 31 & 31 \\
\hline 30 & 30 \\
\hline 40 & 36 \\
\hline 45 & 32 \\
\hline 36 & 40 \\
\hline 35 & 34 \\
\hline 37 & 34 \\
\hline 35 & 33 \\
\hline 35 & 38 \\
\hline
\end{tabular}




\begin{tabular}{|c|c|}
\hline \multicolumn{2}{|c|}{$\boldsymbol{V}_{h 0}$} \\
\hline Mag [V] & Angle $\left[^{\circ}\right]$ \\
\hline 34 & 37 \\
\hline 37 & 36 \\
\hline 37 & 35 \\
\hline 32 & 34 \\
\hline 35 & 32 \\
\hline 37 & 35 \\
\hline 32 & 36 \\
\hline 36 & 38 \\
\hline 39 & 36 \\
\hline 36 & 35 \\
\hline 37 & 34 \\
\hline 33 & 36 \\
\hline 35 & 37 \\
\hline 31 & 35 \\
\hline 30 & 34 \\
\hline 40 & 32 \\
\hline 45 & 34 \\
\hline 41 & 43 \\
\hline 45 & 31 \\
\hline 43 & 32 \\
\hline 43 & 35 \\
\hline
\end{tabular}

